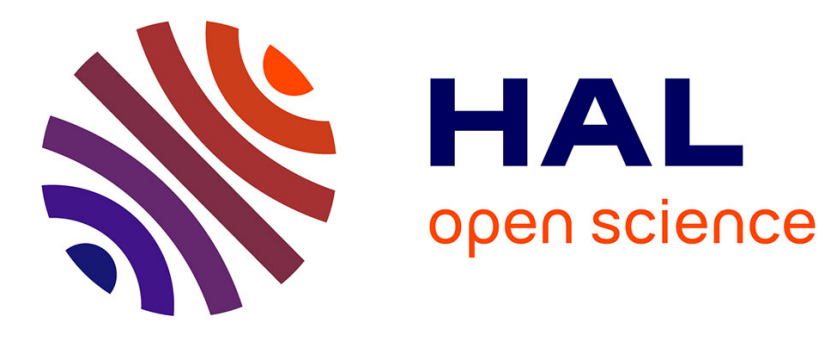

\title{
Sur la géométrie du groupe de Thompson
}

Xavier Martin

\section{To cite this version:}

Xavier Martin. Sur la géométrie du groupe de Thompson. Mathématiques [math]. Université JosephFourier - Grenoble I, 2002. Français. NNT : . tel-00001665

\section{HAL Id: tel-00001665 \\ https://theses.hal.science/tel-00001665}

Submitted on 5 Sep 2002

HAL is a multi-disciplinary open access archive for the deposit and dissemination of scientific research documents, whether they are published or not. The documents may come from teaching and research institutions in France or abroad, or from public or private research centers.
L'archive ouverte pluridisciplinaire HAL, est destinée au dépôt et à la diffusion de documents scientifiques de niveau recherche, publiés ou non, émanant des établissements d'enseignement et de recherche français ou étrangers, des laboratoires publics ou privés. 


\title{
SUR LA GÉOMÉTRIE DU GROUPE DE THOMPSON
}

\author{
Xavier MARTIN
}



$A$ mes parents 



\section{REMERCIEMENTS}

Mes premiers remerciements vont à Yves Carrière. Notre collaboration mathématique me fut bénéfique tout au long de la thèse. C'est lui qui m'a initié à l'intuition géométrique, et qui le premier m'a accordé sa confiance.

Vlad Sergiescu a su prendre le relais. Du groupe de travail "forestier" à ce jour, il a été une source intarissable d'idées et de questions. Son humanité, son courage et sa franchise m'ont galvanisé pendant les moments difficiles. Merci Vlad!

Bill Harvey et Leonid Potyagailo me font l'honneur d'être à la fois rapporteurs et membres du jury, je leur en suis très reconnaissant. Je remercie M. Harvey pour son hospitalité à Londres et M. Potyagailo pour son accueil chaleureux à Lille et ses questions pertinentes sur la dynamique du groupe de Thompson. Merci également à Claude Roger et à Luis Funar pour leur participation amicale à mon jury de thèse.

Je remercie les membres du groupe de travail physique mathématique, Michel Imbert, Christophe Kapoudjian, Guillaume Laget et les autres. J'ai une pensée toute particulière pour Michel, pour nos discussions mathématiques très stimulantes et pour avoir supporté mes différentes humeurs ces derniers mois. Il est indéniablement l'un des artisans de l'aboutissement de ce travail.

Je remercie aussi le personnel administratif de l'Instiut Fourier, notamment Arlette et Bruno pour leur dévouement et leur sympathie.

Je n'oublierai pas mes collègues thésards, dont la liste, non exhaustive, n'a cessé de s'allonger durant ces 3+2 années. Le Nantais et néanmoins Ami Stéphane Sachant Egréner, à point nommé, ses petites touches humoristiques pendant nos pauses-clopes, mes co-bureau 307 fumeurs: p'tit Michel (le corbeau), Valentin (lé Johnny des carpates), Vincent (le polonais), puis non fumeurs: Pierre Emmanuel (Peu, son honneteté et ma santé), Michel (déjà vu) et enfin Ion (Jean Michel). Et les autres: Luc (l'autre Nantais mais surtout l'autre Poitevin), Paolo (Tromba...=Sanita...), Matthieu (ça boum), Costia ( $\backslash$ renewcommand), Laurent (le baroudeur), Helene, Sophie, Grand Stephane, et tous ceux que je n'oublie pas en pensées.

Les amis grenoblois sont aussi de la partie, notamment ceux qui ont bien voulu me supporter au quotidien: Lol, Keltou(n), Mathou le sénégaulois, plus récemment les très généreux "gamins" pti'Seb et Pascale, mais aussi les autres: Simon, Marine et Steph, 
Lise et Did, Olive, gro'Seb, Nadège et Soon, Fleur et Damo, Mathilde et Reza, Jérome et Christelle et bien d'autres encore... Et l'incontournable Tata, obviously.

Je ne saurai trop remercier ma famille, et plus particulièrement mes parents pour leur soutien sans faille tout au long de mon séjour à Grenoble. Vous aussi avez été une source de motivation me permettant de tenir le coup. (Almost) Last but not least, many thanks to my "pretty family", especially to "pretty" Dad with its fortifying words during the last year.

Enfin Jana, je ne serai jamais assez reconnaissant envers toi, pour avoir supporté mon absence au quotidien ces trois dernières années, et pour ta confiance totale en notre amour. Je pense que le sacrifice en valait la peine. 


\section{Table des matières}

$\begin{array}{lll}\text { I Le contexte géométrique } & \mathbf{1 7}\end{array}$

I.1 Combinatoire et topologie . . . . . . . . . . . . . . . . 18

I.2 La géométrie projective par morceaux . . . . . . . . . . . . 23

I.3 Les espaces de bracelets . . . . . . . . . . . . . . . . 26

I.4 L'espace de Teichmüller universel . . . . . . . . . . . . . . 30

I.5 Liens entre les différents contextes géométriques . . . . . . . . . . 33

I.6 Le groupe de Thompson . . . . . . . . . . . . . . . . . 35

II Les systèmes de coordonnées sur $\mathcal{G} r$ et $\mathcal{B} r \quad 39$

II.1 Coordonnées log-lambda . . . . . . . . . . . . . . . . . . . . . . . . 40

II.2 Coordonnées horocycliques . . . . . . . . . . . . . . . . 47

II.3 Une métrique $\operatorname{sur} \mathcal{G} r \ldots \ldots \ldots \ldots \ldots \ldots \ldots$

II.4 Quelques questions de topologie . . . . . . . . . . . . . 53

III Géométrie du groupe de Thompson $\quad \mathbf{5 9}$

III.1 L'action du groupe de Thompson sur $\mathcal{G} r$ et $\mathcal{C} r \ldots \ldots \ldots . . . . .59$

III.2 L'action sur les coordonnées log-lambda . . . . . . . . . . . . 60

III.3 Etude des orbites . . . . . . . . . . . . . . . . . . 62

III.4 Le groupe $T$ est un groupe d'isométries . . . . . . . . . . . . . 65

III.5 Etude des stabilisateurs . . . . . . . . . . . . . . . 68

III.6 Analogie avec les espaces de Teichmüller . . . . . . . . . . . . . . . . 71

III.7 Un classifiant explicite pour $T$. . . . . . . . . . . . . . 72

IV Homologie du groupe de Thompson et espaces de lacets $\quad 75$

IV.1 Le théorème de Ghys et Sergiescu . . . . . . . . . . . . . . . 76

IV.2 Le cas du groupe $F^{\prime} \ldots \ldots \ldots \ldots \ldots . \ldots \ldots 79$

IV.3 Modèle(s) de Cohen de $\Lambda \Sigma X \ldots \ldots \ldots$. . . . . . . . . . . 81

IV.4 Un nouveau modèle de $\Lambda \mathbb{S}^{3} \ldots \ldots \ldots$. . . . . . . . . . . 84

IV.5 Une application de $B \widetilde{T}$ dans $\Lambda \mathbb{S}^{3} \ldots \ldots \ldots \ldots$

IV.6 L'isomorphisme en homologie entière . . . . . . . . . . . . . . 103

IV.7 Le cas du groupe $F^{\prime}$ : une nouvelle preuve . . . . . . . . . . . . . . 112 


\section{Introduction}

\section{Le groupe de Thompson $T$}

Définition 1. (Groupe de Thompson: version PL)

Le groupe $P L_{2}\left(\mathbb{S}^{1}\right)$ est l'ensemble des homéomorphismes $f$ de $\mathbb{R} / \mathbb{Z}$ vérifiant les propriétés suivantes:

1. $f$ est affine par morceaux, avec un nombre fini de morceaux dans $\mathbb{R} / \mathbb{Z}$.

2. Les points de discontinuité de $f$ sont dyadiques (c.a.d. de la forme $\frac{a}{2^{b}}$ où $a$ et $b$ sont des entiers relatifs).

3. Les dérivées à gauche et à droite sont des puissances de 2 en tout point.

4. $f(0)$ est un nombre dyadique.

Voici deux éléments de $P L_{2}\left(\mathbb{S}^{1}\right)$ donnés par leur graphe sur la figure suivante:
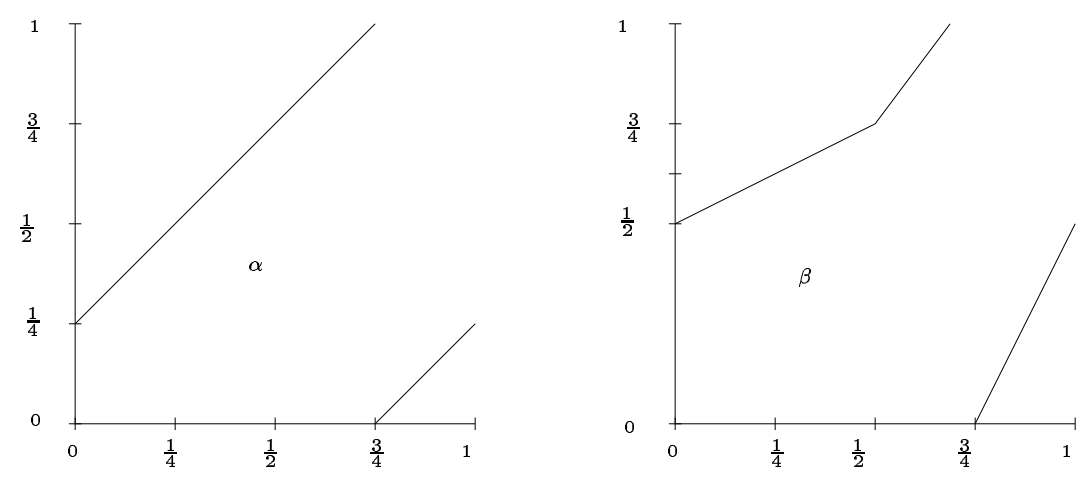

Fig. 1 - Deux éléments version PL.

Le premier élément est constitué d'un unique morceau et le second de trois morceaux. Le groupe $P L_{2}\left(\mathbb{S}^{1}\right)$ est la version linéaire par morceaux (dite PL) du groupe de Thompson $T$.

Définition 2. (Groupe de Thompson: version CPP)

Le groupe $P P S L_{2} \mathbb{Z}$ est l'ensemble des homéomorphismes $f$ de $\mathbb{S}^{1}$ de type $P S L_{2} \mathbb{Z}$ par morceaux, à points de coupure rationnels et en nombre fini. 

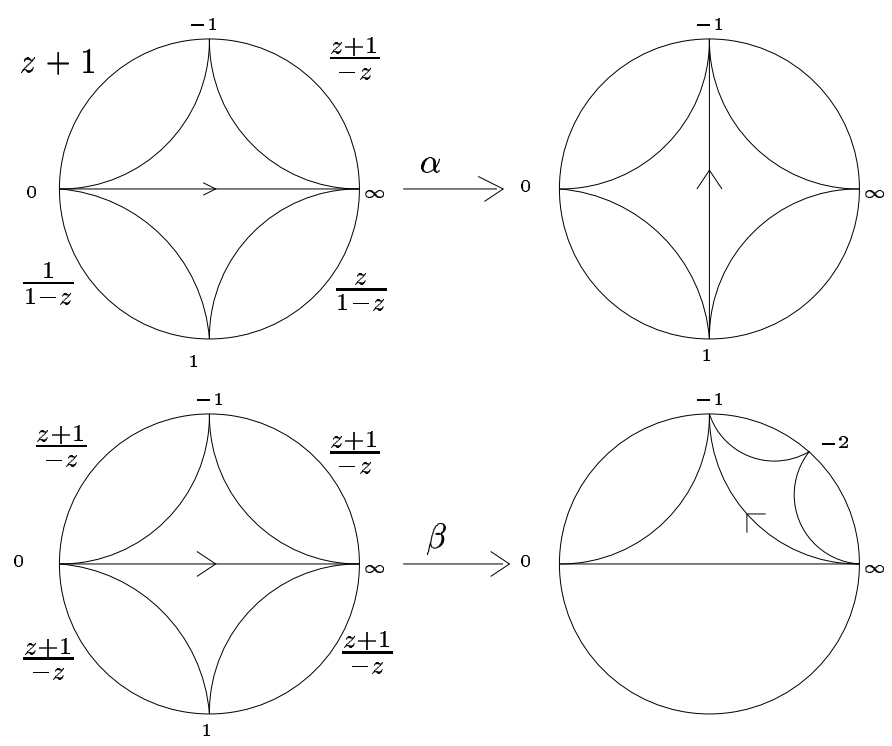

Fig. 2 - Deux éléments version $C P P$.

Le premier élément est constitué de quatre morceaux et le second est dans $P S L_{2} \mathbb{Z}$. Le groupe $P P S L_{2} \mathbb{Z}$ est la version projective par morceaux et de classe $\mathcal{C}^{1}$ (dite CPP) du groupe $T$.

On définit également $F$ (resp. $F^{\prime}$ ) comme le sous-groupe de $T$ fixant 0 (ou $\infty$ ) (resp. le sous-groupe des éléments dont le germe en 0 (ou $\infty$ ) est trivial). Enfin, le relevé de $T$ sur la droite réelle sera noté $\widetilde{T}$. Il donne l'extension centrale de groupes:

$$
0 \rightarrow \mathbb{Z} \rightarrow \widetilde{T} \rightarrow T \rightarrow 0
$$

\section{Développements sur les groupes $T$ et $F$}

Apparu avec le problème des mots, le groupe de Thompson $T$ est le premier exemple de groupe infini simple, de présentation finie [McK-Tho]. L'étude des groupes $T$ et $F$ a suscité un intéret constant depuis leur introduction, dans une note de R. Thompson, non publiée [Tho]. Elle apparait progressivement dans des domaines variés des mathématiques, tels que la théorie des groupes, la théorie de l'homotopie, les systèmes dynamiques et la logique. Une version plus combinatoire de ces groupes se définit en terme de diagrammes d'arbres, provenant de la structure binaire des nombres dyadiques.

Les représentations "PL" et "diagrammes d'arbres" seront les principales utilisées jusqu'à la fin des années 80 . La version des diagrammes d'arbres donne des résultats sur la structure de groupe de $F$ et $T$. Dans [Can-Flo-Par], les auteurs fournissent une démonstration accessible de la simplicité du groupe $T$ (résultat existant déjà dans [Tho]). La version PL permet notamment de trouver des propriétés homotopiques 
et homologiques de finitude des groupes de Thompson, comme la propriété $F P_{\infty}$ et l'égalité $H^{*}(G ; \mathbb{Z} G)=0$ pour un tel groupe $G$ [Bro-Geo].

D'un point de vue dynamique, ces groupes d'homéomorphismes possèdent de bonnes propriétés. Elles sont étudiées dans [Ghy-Ser]. Par exemple, le groupe $T$ peut être "lissé", c'est à dire conjugué à un groupe de difféomorphismes du cercle de classe $\mathcal{C}^{\infty}$. Dans ce même article, la cohomologie entière des groupes $F, \widetilde{T}$ et $T$ est reliée à celle des espaces de lacets sur la sphère $\mathbb{S}^{3}$, dans le théorème suivant:

Théorème 1. (Théorème IV.1)(Ghys-Sergiescu,[Ghy-Ser]) Il existe des applications continues

$$
\begin{aligned}
& B F^{\prime} \stackrel{a}{\longrightarrow} \Omega \mathbb{S}^{3} \\
& B \widetilde{T} \stackrel{b}{\longrightarrow} \Lambda \mathbb{S}^{3} \\
& B T \stackrel{c}{\longrightarrow} \mathcal{L S}^{3}
\end{aligned}
$$

qui induisent des isomorphismes en homologie entière.

\section{Les aspects 2-dimensionnels du groupe $T$}

Dans les années 80, une remarque de Thurston permet de voir le groupe $P L_{2}\left(\mathbb{S}^{1}\right)$ sous un jour nouveau. Il est conjugué (par un homéomorphisme du cercle $i$ ) au groupe des homéomorphismes $P S L_{2} \mathbb{Z}$ par morceaux $P P S L_{2} \mathbb{Z}$. L'homéomorphisme $i$ se définit en prolongeant par densité une bijection naturelle (préservant l'orientation) entre l'ensemble $\mathbb{Q}(2)$ des nombres dyadiques de $\mathbb{R} / \mathbb{Z}$, et l'ensemble $\widehat{\mathbb{Q}}$ des rationnels de la droite projective réelle. Ce n'est qu'au début des années 90, que les aspects 2-dimensionnels du groupe de Thompson sont apparus. Plusieurs travaux vont dans ce sens, notamment:

1. L'étude de l'action du groupe $P P S L_{2} \mathbb{Z}$ sur certains espaces d'homéomorphismes du cercle de classe CPP et sur des espaces de bracelets: (P. Greenberg [Gre3],[Gre4] 1992).

2. L'étude de l'action du groupe de Ptolémée $G$ sur l'espace des tesselations (R. C. Penner [Pen2] 1993).

3. Les connections des groupes $F$ et $T$ avec le groupe des tresses infini $B_{\infty}$ : (P. Greenberg, V. Sergiescu [Gre-Ser]).

Nous n'aborderons pas ce dernier point de vue. Rappelons cependant que les auteurs établissent une extension acyclique $A$ du groupe des tresses infini sur le groupe $F^{\prime}$. Leur méthode de construction de $A$ est plutot géométrique et utilise une action de $F^{\prime}$ agissant au voisinage de l'infini sur l'arbre binaire.

Nous devons quelques explications sur le vocabulaire employé. Celui-ci fournit une idée de la méthode employée dans la thèse.

a. Projectif par morceaux: Un homéomorphisme du cercle $f$ est dit projectif par morceaux (ou PP) si il existe une subdivision finie de $\mathbb{S}^{1}$ en intervalles, telle que $f$ 
est de type $P S L_{2} \mathbb{R}$ sur chaque intervalle. Le groupe de Thompson $T$ est un groupe d'homéomorphismes PP.

b. La condition $\mathcal{C}^{1}$ : La nature arithmétique de $P P S L_{2} \mathbb{Z}\left(P S L_{2} \mathbb{Z}\right.$ est définie par l'équation $a d-b c=1$ dans $\left.\mathbb{Z}^{4}\right)$ impose de la rigidité au niveau différentiable. Les éléments de $P P S L_{2} \mathbb{Z}$ sont de classe $\mathcal{C}^{1}$ (proposition I.8).

Ainsi le groupe $T$ se plonge dans l'espace $C P P$ (resp. $\left.C P P_{\mathbb{Q}}\right)$ des homéomorphismes $\mathrm{PP}$ de classe $\mathcal{C}^{1}$ (resp. et à points de coupure rationnels). Quel est l'interet de la condition $\mathcal{C}^{1}$ ? Il tient dans l'observation suivante. Le groupe $C P P$ agit à gauche sur l'ensemble des horocycles du demi-plan de Poincaré (corollaire I.1).

c. Tesselation décorée: Une tesselation décorée est une triangulation idéale du disque de Poincaré munie d'une collection d'horocycles (la décoration) portée par l'ensemble des sommets et d'une arête orientée distinguée.

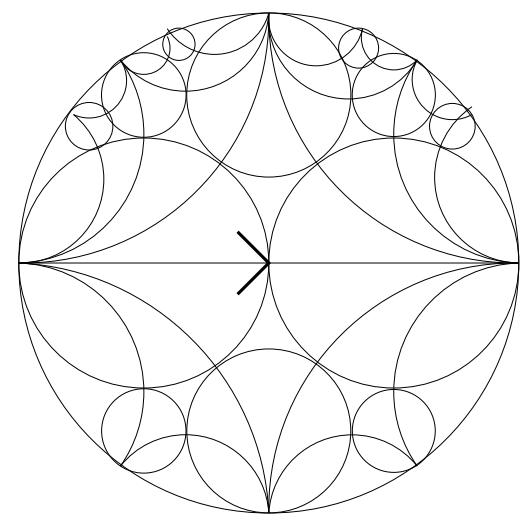

FIG. 3 - Tesselation décorée de Farey-Ford.

L'observation précédente nous permet de pousser la tesselation décorée de FareyFord (figure 3), notée $\tilde{\tau}_{*}$, par les homéomorphismes de classe CPP. Notons $\widetilde{\text { Tess l'espace }}$ des tesselations décorées. L'arête orientée distinguée fixe la combinatoire des tesselations décorées. A chaque arête $e^{\prime}$ d'une tesselation décorée $\tilde{\tau}$, est associée, d'une part une arête $e$ de $\tau_{*}$, et d'autre part la "distance" des horocycles qu'elle supporte (appelée lambda-longueur de $\tilde{\tau}$ le long de $e^{\prime}$. Ces deux propriétés se combinent pour donner le:

Théorème 2. (Théorème II.1) (Penner,[Pen2]) Il existe un plongement E de $\widetilde{\text { Tess }}$ dans l'espace des paramètres $\mathbb{R}_{+}^{*} \tau_{*}$.

Ainsi l'espace $\widetilde{\text { Tess }}$ est muni d'un système de coordonnées affine global. Ses paramètres sont appelés coordonnées log-lambda.

\section{Les contributions de Greenberg}

Nous nous référons aux notes [Gre4] et à l'appendice de l'article [Gre3]. Ils fournissent l'entame d'une étude des groupes de Thompson à l'aide de la géométrie CPP. 
L'auteur étudie notamment l'action du groupe $P P S L_{2} \mathbb{Z}$ par composition à droite sur l'espace de dimension infinie suivant:

$\mathcal{G} r=P S L_{2} \mathbb{R} \backslash\{$ éléments de $C P P$ à points de coupure rationnels $\}=P S L_{2} \mathbb{R} \backslash C P P_{\mathbb{Q}}$

L'action possède les propriétés suivantes:

1. Les orbites sont discrètes.

2. Les stabilisateurs sont cycliques finis ou conjugués à $P S L_{2} \mathbb{Z}$.

3. l'action vérifie une propriété de faible discontinuité:

$$
\forall f \in \mathcal{G} r, \exists V_{f} \text { un voisinage ouvert de } f \mid V_{f} \cdot \gamma \cap V_{f} \neq \emptyset \Rightarrow \gamma \text { fixe } f \text {. }
$$

P.Greenberg esquisse une démonstration de la contractibilité de $\mathcal{G} r$ (proposition 0.3.2.1 [Gre4]). On obtient comme corollaire que le quotient $\mathcal{G} r / F^{\prime}$ est un classifiant du groupe $F^{\prime}$. La preuve de la contractibilité utilise un outil géométrique appelé bracelet (un $n$-bracelet est une collection de $n$ horocycles $h_{1}, \cdots, h_{n}$ tels que $h_{i}$ et $h_{i+1}$ sont tangents $\forall i \in \mathbb{Z} / n \mathbb{Z}$ ).

L'appendice de [Gre3] fournit, sans preuve, un système de coordonnées de l'espace

$$
\mathcal{C}_{n}=P S L_{2} \mathbb{R} \backslash\{(n+1)-\text { bracelets }\}=P S L_{2} \mathbb{R} \backslash \widehat{\mathcal{C}_{n}}
$$

Ce sont les longueurs (une par horocycle) des segments d'horocycles délimités par les points de tangence. Ces coordonnées "horocycliques" servent à la construction d'une application continue

$$
\varphi: \mathcal{G} r / F^{\prime} \rightarrow \Omega \mathbb{S}^{2}
$$

qui se relève en une application $\tilde{\varphi}$ à valeurs dans $\Omega \mathbb{S}^{3}$ vérifiant le:

Théorème 3. (théorème IV.5) L'application $\tilde{\varphi}$ donne un isomorphisme en homologie entière.

L'esquisse de démonstration de Greenberg utilise la connaissance, à priori, de la structure multiplicative de l'homologie du groupe $F^{\prime}$, isomorphe à celle de $\Omega \mathbb{S}^{3}$. L'application $\tilde{\varphi}$ réalise ainsi la flèche $a$ du theoréme 1.

\section{Le contenu de la thèse}

Le premier objectif de cette thèse est de comprendre le preprint [Gre4], et plus spécifiquement ses retombées sur la géométrie du groupe de Thompson $P P S L_{2} \mathbb{Z}$. En "poussant" la tesselation décorée de Farey-Ford $\tilde{\tau}_{*}$ par les homéomorphismes CPP, on établit un pont entre les articles [Gre4] et [Pen2]. Notre démarche consiste à interpréter les résultats de Greenberg dans le système de coordonnées de Penner (théorème 2), afin d'en soustraire des preuves complètes et conceptuelles des résultats principaux de [Gre4].

Notre deuxième objectif est de trouver une interprétation géométrique directe du théorème 1 dans le contexte CPP. Il s'agit en quelque sorte, d'une extension du théorème 3 . 
Le plan est organisé de la façon suivante.

I - On rappelle les différents contextes géométriques utilisés dans la thèse:

- La géométrie projective par morceaux, dite "CPP" et les espaces de bracelets apparaissant dans [Gre3] et [Gre4].

- La théorie des espaces de Teichmüller décorés de Penner [Pen2].

On y introduit notamment l'espace $\mathcal{G} r$, certains espaces de bracelets, et le groupe de Thompson $T=P P S L_{2}(\mathbb{Z})$.

Le lien entre ces deux contextes est mis en évidence par la:

Proposition A. (Proposition I.6) L'application

$$
s: f \in P S L_{2}(\mathbb{R}) \backslash C P P \mapsto f \cdot \tilde{\tau}_{*} \in \widetilde{\text { Tess }}
$$

fournit une injection continue pour la topologie $\mathcal{C}^{1}$ sur $C P P$.

II - Ce chapitre se décompose en deux parties. Dans la section II.1, on utilise le système de coordonnées donné par le théorème 2 (ci-dessus), en restriction à l'espace $\mathcal{G} r$. Soit $\Lambda=E \circ s$ et notons $\left(\mathbb{R}_{+}^{*}\right)_{\sqrt{2}}^{\tau_{*}}$ l'ensemble des suites à termes strictement positifs stationnaires en $\sqrt{2}$.

Théorème B. (Théorème II.2) L'application $\Lambda$ réalise un homéomorphisme de $\mathcal{G} r$ sur $\left(\mathbb{R}_{+}^{*}\right)_{\sqrt{2}}^{\tau_{*}}$.

On déduit une preuve conceptuelle de la contractibilité de $\mathcal{G} r$ (proposition 0.3.2.1 dans [Gre4]). Les lambda-longueurs permettent également de paramêtrer l'espace des $(n+1)$-bracelets propres modulo $P S L_{2} \mathbb{R}$, noté $\mathcal{B}_{n}$ (un bracelet est propre si aucun couple d'horocycles du bracelet ne s'intersecte transversalement). Dans [Gre3], l'auteur montre que l'espace $\mathcal{B}_{n}$ est homéomorphe au polytope de Stasheff $K_{n}$. Ainsi, l'espace $\mathcal{B}_{n}$ est contractile. Nous donnons une nouvelle démonstration de la contractibilité de $\mathcal{B}_{n}$ à l'aide des coordonnées log-lambda (théorème II.4).

La deuxième partie du chapitre II (sections II.2 et II.3) est consacré à l'étude de l'appendice dans [Gre3]. A l'aide d'un système de coordonnées "angulaires" (les $h$-coordonnées définies sur $\widetilde{T e s s}$ ), on fournit une démonstration du "Résultat" A. 1. énoncé dans [Gre3]. Soit $\mathcal{C}_{n}$ l'espace des $(n+1)$-bracelets modulo $P S L_{2} \mathbb{R}$. Pour un bracelet $b=\left(h_{i}\right)_{i \in<1 ; n+1>}$, on note $l_{i}(b)$ la longueur du segment de $h_{i}$ délimité par ses intersections avec $h_{i-1}$ et $h_{i+1}$. On obtient ainsi un plongement

$$
p_{n}: b \in \mathcal{C}_{n} \mapsto\left(l_{i}(b)\right)_{i \in<1 ; n+1>} \in\left(\mathbb{R}_{+}^{*}\right)^{n+1}
$$

L'image de ce plongement est paramétrée comme suit:

Proposition C. (Proposition II.3) L'application:

$$
\begin{aligned}
r_{n}:\left(\mathbb{R}_{+}^{*}\right)^{n-2} & \rightarrow \\
\left(\sigma_{3}, \cdot, \sigma_{n}\right) & \mapsto\left(\frac{1}{\sigma_{3}}+\sum_{j=2}^{n-2} \frac{1}{\sigma_{j+1} \sigma_{j+2}}+\frac{1}{\sigma_{n}}, \sigma_{3}, \frac{1+\sigma_{4}}{\sigma_{3}}, \cdot, \frac{\sigma_{i-1}+\sigma_{i+1}}{\sigma_{i}}, \cdot \frac{1+\sigma_{n-1}}{\sigma_{n}}, \sigma_{n}\right)
\end{aligned}
$$

est un plongement dont l'image paramêtrise $p_{n}\left(\mathcal{C}_{n}\right)$. 
La suite d'applications $\left(p_{3 \cdot 2^{n}-1}\right)_{n \geq 0}$ fournit un autre système de coordonnées sur l'espace $\mathcal{G} r$, à valeurs dans une limite directe de $\left(\mathbb{R}^{3 \cdot 2^{n}}\right)_{n \geq 0}$. L'interet de celui-ci est qu'il fournit aussi une métrique, noté $d^{b}$ sur $\mathcal{G} r$. On verra au chapitre III qu'elle est invariante par le groupe de Thompson.

III - Ce chapitre est consacré à l'action du groupe de Thompson $T$ sur l'espace contractile $\mathcal{G} r$. A l'aide de la formule de Ptolémée (proposition III.2) décrite dans [Pen1], nous précisons le lieu de l'orbite d'un élément $f$ de $\mathcal{G} r$ dans l'espace des coordonnées $\left(\mathbb{R}_{+}^{*}\right)_{\sqrt{2}}^{\tau_{*}}$ en fonction des coordonnées de $f$. On obtient, avec une méthode nouvelle, que l'action est discrète. Plus précisément si $f \in \mathcal{G} r$ et $A=\left\{\Lambda(f)(e) ; e \in \tau_{*}\right\}$ (c'est un ensemble fini), notons $N(A)$ l'ensemble des combinaisons linéaires, à coefficients entiers positifs et non tous nuls, d'éléments de $A$. C'est un ensemble discret de $\mathbb{R}_{+}^{*}$. On définit à partir de $A$ le sous-ensemble discret de $\left(\mathbb{R}_{+}^{*}\right)_{\sqrt{2}}^{\tau_{*}}$ suivant:

$$
\left(\mathbb{R}_{+}^{*}\right)_{A}^{\tau_{*}}=\left\{\Lambda \in\left(\mathbb{R}_{+}^{*}\right)_{\sqrt{2}}^{\tau_{*}} \mid \Lambda(e) \in N(A) \quad \forall e \in \tau_{*}\right\}
$$

Proposition D. (proposition III.3) L'orbite de $f$ est contenue dans $\left(\mathbb{R}_{+}^{*}\right)_{A}^{\tau_{*}}$. En particulier $T$ agit avec orbites discrètes.

Une autre démonstration de cette proposition est donnée dans le cadre des coordonnées horocycliques. Elle utilise la métrique $d^{b}$. Par ailleurs, cette métrique est adaptée à l'action du groupe de Thompson comme le montre la:

Proposition E. (proposition III.4) Le groupe T agit isométriquement sur l'espace $\mathcal{G r}$ muni de la métrique $d^{b}$.

Comme application directe de ce résultat, nous obtenons la propriété de faible discontinuité (énoncée plus haut) de l'action du groupe $T$ (proposition III.6).

Concernant l'étude des stabilisateurs, nous reprenons essentiellement la démarche de Greenberg, en évitant cependant l'utilisation de la rationnalité des nombres de rotations des éléments de $T$ (voir [Ghy-Ser]), qui est un résultat assez délicat.

Enfin, nous étendons la construction du classifiant du groupe $F^{\prime}$ au groupe $T$, en passant au revêtement universel du cercle. On obtient successivement des espaces classifiants $B \widetilde{T}$ et $B T$. Ceux-ci forment une fibration en cercle

$$
\mathbb{S}^{1} \rightarrow B \widetilde{T} \rightarrow B T
$$

dont la suite exacte d'homotopie donne l'extension centrale de $\widetilde{T}$ sur $T$.

IV - Ce chapitre sera consacrée à l'homologie des groupes de Thompson et notamment à leurs connexions avec les espaces de lacets de la sphère $\mathbb{S}^{3}$. Nous construisons des versions géométriques des applications $a b$ et $c$ du théorème 1 .

Après un rapide survol géométrique de la démonstration de [Ghy-Ser], nous expliquons rapidement la méthode de Greenberg pour définir l'application $\tilde{\varphi}$. Il utilise le modèle de James $J \mathbb{S}^{1}=\coprod_{n \in \mathbb{N}}\left(\mathbb{S}^{1}\right)^{n} / \sim$ (où $\sim$ est une relation à définir) de $\Omega \mathbb{S}^{2}$ [Jam] pour construire l'application $\varphi$ à valeurs dans $J \mathbb{S}^{1} \simeq \Omega \mathbb{S}^{2}$ qu'il relève à $\Omega \mathbb{S}^{3}$. 
Pour interpréter l'application $b$, nous développons un nouveau modèle combinatoire de $\Lambda \mathbb{S}^{3}$, noté $M \mathbb{S}^{2}$ (section IV.4). La construction de $M \mathbb{S}^{2}$ se fait à partir du modèle de Cohen de $\Lambda \mathbb{S}^{2}$ [Coh], et on montre que $M \mathbb{S}^{2}$ est homotopiquement équivalent à $\Lambda \mathbb{S}^{3}$ (corollaire IV.2). Ce modèle nous semble intéressant en soi, mais sa définition assez technique ne nous permet pas de le décrire dans l'introduction.

Nous utilisons ce modèle pour construire une application $\psi: B \widetilde{T} \rightarrow \Lambda \mathbb{S}^{3}$ (section IV.5). Celle-ci fait intervenir la primitive $\theta$ d'un 1-cocycle nul $[\mu]$ du groupe $T$ à valeurs dans le $T$-module $\mathcal{C}^{0}\left(C P P_{\mathbb{Q}} ; \mathbb{S}^{1}\right)$. Puis, nous montrons, en utilisant la structure multiplicative de l'homologie du groupe $\widetilde{T}$, le résultat principal de ce dernier chapitre: Théorème F. (Théorème IV.11) L'application $\psi$ fournit un isomorphisme en homologie entière.

La démonstration du théorème IV.11 utilise le fait que $\psi$ est $\mathbb{S}^{1}$-équivariante, ce qui équivaut, dans la construction de $\psi$, à l'invariance de $\theta$ par $\mathbb{S}^{1}$. Nous n'avons su exhiber une telle primitive $\theta$, mais nous espérons la calculer dans un proche avenir. Nous admettrons donc la condition suivante naturelle:

- $\left(\mathbb{S}^{1}-\mathrm{INV}\right)$ : "Il existe une primitive de $[\mu]$ invariante par l'action du cercle".

Puis, par le procédé de construction de Borel, on obtient l'application $\psi \times_{\mathbb{S}^{1}}$ id : $B T \rightarrow \mathcal{L} \mathbb{S}^{3}=\Lambda \mathbb{S}^{3} \times_{\mathbb{S}^{1}} E \mathbb{S}^{1}$. Celle-ci donne un isomorphisme en homologie entière (corollaire IV.7). C'est une version géométrique de l'application $c$.

Enfin, dans la section IV.7, une approche quelque peu différente est développée. Nous redémontrons le théorème 3 en utilisant la seule connaissance de l'homologie de $F$. Cette approche repose sur l'existence d'un produit (non associatif) sur $\mathcal{G r} / F^{\prime}$ rendant l'application $\varphi$ multiplicative $\left(J \mathbb{S}^{1}\right.$ est muni du produit de la concaténation des mots). 


\section{Chapitre I}

\section{Le contexte géométrique}

Dans ce chapitre, nous introduisons les objets géométriques qui permettent d'aborder les aspects 2-dimensionnels du groupe de Thompson $T$.

Après un bref rappel de géométrie hyperbolique, nous donnons deux objets importants: la triangulation de Farey $\tau_{*}$, et le bracelet de Ford $b^{F}$. La triangulation de Farey permet de comprendre la structure dyadique de l'ensemble des rationnels du cercle $\widehat{\mathbb{Q}}$. On decrit ainsi une bijection naturelle entre les rationnels et les dyadiques du cercle $i: \widehat{\mathbb{Q}} \rightarrow \mathbb{Q}(2)$ préservant l'orientation. Nous rappelons également quelques résultats sur les limites inductives d'espaces topologiques.

Dans la section I.2, on définit les homéomorphismes de classe CPP et l'espace $\mathcal{G} r$ (définition I.2.3) en suivant [Gre4]. Des exemples de tels homéomorphismes, les hyperboliques généralisés, sont donnés.

La section I.3 décrit les bracelets, sortes d'analogues géométriques des homéomorphismes de classe CPP.

La section I.4 fournit un survol rapide de l'espace des tesselations (décorés) Tess $(\widetilde{T e s s})$ introduit par Penner dans sa recherche d'un espace de Teichmüller universel. Soulignons qu'un cas particulier des hyperboliques généralisés, appelé déformation loglambda, apparait dans nombre de démonstrations de [Pen2].

Dans la section I.5, nous fournissons un lien entre la section I.4 et les sections I.2 et I.3 (proposition I.6). Il s'agit d'une injection continue de l'espace $P S L_{2} \mathbb{R} \backslash C P P$ dans l'espace des tesselations décorées. C'est la principale nouveauté dans le cadre de ce chapitre.

Enfin, dans la section I.6, nous rappelons les différentes versions du groupe de Thompson dans les contextes étudiés auparavant. Nous donnons quelques indications des isomorphismes entre les différentes représentations du groupe $T$ qui nous serviront dans les deux derniers chapitres de la thèse. 


\section{I.1 Combinatoire et topologie}

L'objet de cette section est la mise en place des outils élémentaires que nous utiliserons fréquemment dans la suite du texte. Après un bref descriptif de la géométrie hyperbolique du demi-plan de Poincaré, on rappelle deux objets arithmétiques et géométriques important: la triangulation de Farey, et la collection des horocycles de Ford. On introduit au passage l'énumération de $\widehat{\mathbb{Q}}=\mathbb{Q} \cup\{\infty\}$ par les nombres de Farey.

On décrit ensuite comment construire une triangulation idéale quelconque du disque de Poincaré. Cette construction met en lumière la combinatoire dyadique commune à ces triangulations.

Enfin, quelques propriétés des limites inductives d'espaces topologiques sont rappelées. Deux topologies sur une telle limite y sont décrites.

\section{I.1.a La géométrie hyperbolique en jeu}

La géométrie du plan hyperbolique réel (géométrie uniformisant les surfaces dont la caractéristique d'Euler est négative) possède de nombreux modèles de description. Nous utiliserons essentiellement les deux modèles les plus communs. Pour des considérations analytiques, nous prendrons pour modele le plan hyperbolique

$$
\mathbb{H}^{2}=\{z \in \mathbb{C} \mid \operatorname{Im}(z)>0\}
$$

muni de la métrique de Poincaré $d s^{2}=\frac{d x^{2}+d y^{2}}{y^{2}}$. Le groupe des isométries de $\mathbb{H}^{2}$ préservant l'orientation de $\mathbb{H}^{2}$ est $P S L_{2} \mathbb{R}$ et agit par transformations homographiques $z \mapsto \frac{a z+b}{c z+d}$ avec $a d-b c=1$. Cependant, dans la plupart des raisonnements géométriques et pour les figures qui accompagnent ces raisonnements, nous adopterons le point de vue du disque de Poincaré $\mathbb{D}^{2}=\{z \in \mathbb{C} \quad|\quad| z \mid \leq 1\}$.

Concernant le bord à l'infini, on note généralement

$$
\partial \mathbb{D}^{2}=\mathbb{S}^{1} \text { et } \partial \mathbb{H}^{2}=\mathbb{R} \cup\{\infty\}
$$

Via la transformation de Cayley entre les modèles $\mathbb{H}^{2}$ et $\mathbb{D}^{2}$,

$$
\varphi: z \in \mathbb{H}^{2} \mapsto \frac{z-i}{z+i} \in \mathbb{D}^{2}
$$

qui est une isométrie conforme, nous parlerons, par abus de langage, de l'action de $P S L_{2} \mathbb{R}$ sur le cercle $\mathbb{S}^{1}$.

Par ailleurs, $\widehat{\mathbb{Q}}$ désignera l'ensemble des rationnels, infini compris, du cercle $\mathbb{R} \cup$ $\{\infty\}$. Ainsi, un élément de $\widehat{\mathbb{Q}}$ s'ecrira souvent sous la forme $\frac{a}{b}$ avec $a$ et $b$ premiers entre eux et $b>0$ et l'infini sera dénoté $\frac{1}{0}$. La géométrie hyperbolique possède d'autres modèles, mais nous ne les décrirons pas.

Un troisième modèle pour le cercle sera $\mathbb{R} / \mathbb{Z}$. Dans ce modèle, on utilisera l'ensemble $\mathbb{Q}(2)=\left\{\frac{p}{2^{q}} \in \mathbb{R} / \mathbb{Z} \mid(p, q) \in \mathbb{Z}^{2}\right\}$ des nombres dyadiques du cercle.

Par simplicité, quelque soit le modèle utilisé, on notera le bord à l'infini $\mathbb{S}^{1}$ (sauf mention expresse). 


\section{I.1.b Triangulation de Farey - énumeration de $\widehat{\mathbb{Q}}$}

Avec les notations précédentes, la tranformation de Cayley envoie les rationnels $\frac{0}{1}$ (resp. $\frac{1}{0},-\frac{1}{1}$ ) sur -1 (resp. $1, i$ ) dans $\mathbb{S}^{1}$. Ces trois points forment un triangle idéal $T_{0}$ de $\mathbb{D}^{2}$, c'est à dire un ouvert délimité par trois géodésiques se rencontrant deux à deux sur le bord à l'infini.

Considérons l'orbite de $T_{0}$ par $P S L_{2} \mathbb{Z}$. C'est la triangulation de Farey. Elle décompose le disque de Poincaré en triangles idéaux. On la notera $\tau_{*}$.

Comment peut on obtenir une triangulation idéale de $\mathbb{D}^{2}$ ? On construit un premier triangle $T_{0}$ il reste alors à trianguler les composantes connexes de $\mathbb{D}^{2} \backslash T_{0}$. On construit donc un triangle dans chaque composante connexe de sorte que chaque nouveau triangle a une arête provenant de $T_{0}$. Cela donne trois nouveaux triangles $T_{1}, T_{2}, T_{3}$. On recommence ce procédé par induction.
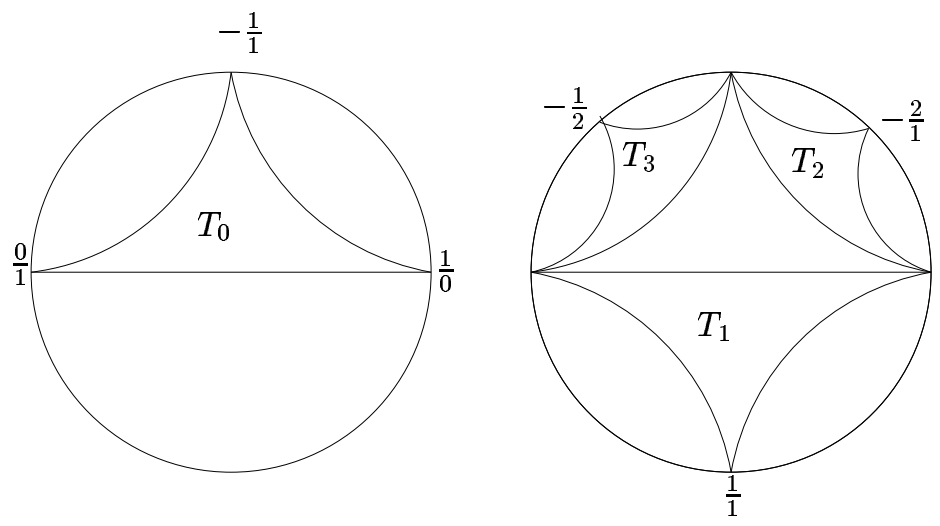

FIG. I.1 - Triangulation de Farey partielle.

A l'étape $n$, on construit $3 \cdot 2^{n}$ nouveaux triangles et le nombre de sommets de la triangulation partielle obtenue après l'ajout de ces $3 \cdot 2^{n}$ triangles est exactement $3 \cdot 2^{n+1}$. Pour obtenir une vraie triangulation de $\mathbb{D}^{2}$ on doit appliquer indéfiniment ce procédé. Remarquons que la combinatoire mise en jeu dans la construction est indépendante des triangles choisis, à partir du moment ou l'on a construit $T_{0}$ et $T_{1}$. Elle est de type dyadique.

L'ensemble $\widehat{\mathbb{Q}}$ représente donc également l'ensemble des extrémités des géodésiques de la triangulation de Farey. On assigne les extrémités, dont la partie imaginaire est négative, aux rationnels positifs de la manière suivante:

Si $T$ est un élément de la triangulation, et deux de ses sommets sont $\frac{a}{b}$ et $\frac{c}{d}$, avec $a \wedge b=1$ et $c \wedge d=1$, alors le troisième sommet de $T$ est assigné à la valeur $\frac{a}{b} \oplus \frac{c}{d}=\frac{a+c}{b+d}$. On appelle $\operatorname{Max}(|a|,|b|)$ l'ordre d'une fraction irréductible $\frac{a}{b}$. On peut alors assigner les extrémités de $\tau_{*}$ par recurrence sur l'ordre défini sur $\widehat{\mathbb{Q}}$. Par exemple $\frac{1}{1}$ s'envoye sur $-i$ et forme avec $\frac{0}{1}$ et $\frac{1}{0}$ un triangle de la triangulation. On fait ensuite la même construction pour les extrémités dont la partie imaginaire est positive, ou bien on conjugue la situation, c'est à dire que si $z$ est assigné à $\frac{a}{b}$, on assigne $\bar{z}$ à $-\frac{a}{b}$. 
Nous filtrons $\widehat{\mathbb{Q}}$ par les sous ensembles $\left(S_{n}\right)_{n \in \mathbb{N}^{*}}$, définis par récurrence:

$$
S_{1}=\left\{\frac{0}{1} ; \frac{1}{0} ;-\frac{1}{1}\right\}
$$

et supposons $S_{n}$ construit, on définit $S_{n+1}$ comme étant $S_{n} \cup A_{n}$, où $A_{n}$ est l'ensemble des sommets des triangles dont les deux autres sommets sont dans $S_{n} \backslash S_{n-1}$. On obtient ainsi:

$$
\begin{gathered}
S_{2}=\left\{\frac{0}{1} ; \frac{1}{0} ;-\frac{1}{1} ; \frac{1}{1} ;-\frac{2}{1} ;-\frac{1}{2}\right\} \\
S_{3}=\left\{\frac{0}{1} ; \frac{1}{0} ;-\frac{1}{1} ; \frac{1}{1} ;-\frac{2}{1} ;-\frac{1}{2} ; \frac{1}{2} ; \frac{2}{1} ;-\frac{3}{1} ;-\frac{3}{2} ;-\frac{2}{3} ;-\frac{1}{3}\right\}
\end{gathered}
$$

Remarquons que cette filtration fournit un ordre naturel à l'ensemble $\widehat{\mathbb{Q}}$. On note ainsi $u_{1}=\frac{0}{1}, u_{2}=\frac{1}{0}, u_{3}=-\frac{1}{1}, u_{4}=\frac{1}{1}, u_{5}=-\frac{2}{1}, u_{6}=-\frac{1}{2}, u_{7}=\frac{1}{2}, \cdots$.

Elle fournit également un ordre naturel sur $\tau_{*}$ commançant comme suit:

$$
e_{1}=\left(\frac{0}{1} ; \frac{1}{0}\right)<e_{2}=\left(\frac{1}{0} ;-\frac{1}{1}\right)<e_{3}=\left(-\frac{1}{1} ; \frac{0}{1}\right)<e_{4}=\left(\frac{0}{1} ; \frac{1}{1}\right)<\cdots
$$

ainsi qu'un ordre naturel sur l'ensemble des triangles de Farey $T_{0}<T_{1}<T_{2}<T_{3}<\cdots$. La figure I.1 montre les premiers termes de ces suites ordonnées.

\section{I.1.c $\quad$ Dyadiques $=$ Rationnels}

Nous aurons besoin dans la suite d'une bijection naturelle entre les nombres dyadiques et les nombres rationnels du cercle. Cette bijection découle de la structure dyadique des nombres rationnels.

Posons $i\left(\frac{0}{1}\right)=0=1 \in \mathbb{R} / \mathbb{Z}, i\left(\frac{1}{1}\right)=\frac{1}{4}, i\left(\frac{1}{0}\right)=\frac{1}{2}$ et $i\left(-\frac{1}{1}\right)=\frac{3}{4}$. On prolonge $i$ sur l'intervalle $\left[\frac{0}{1} ; \frac{1}{1}\right]$ par récurrence sur l'ordre des rationnels:

Supposons $i$ prolongée sur l'ensemble des rationnels d'ordre au plus $n-1$, de $\left[\frac{0}{1} ; \frac{1}{1}\right]$. Si $q$ est d'ordre $n$, alors il est sommet d'un triangle de Farey, dont les deux autres sommets $\frac{a}{b}$ et $\frac{c}{d}$ sont d'ordre au plus $n-1$. Ainsi, on a $q=\frac{a+c}{b+d}$. On définit alors $i(q)=\frac{i\left(\frac{a}{b}\right)+i\left(\frac{c}{d}\right)}{2} \in\left[i\left(\frac{a}{b}\right) ; i\left(\frac{c}{d}\right)\right]$. On a ainsi défini un prolongement de $i$ sur l'ensemble des rationnels de l'intervalle considéré, à valeurs dans l'ensemble des dydadiques de $\left[0 ; \frac{1}{4}\right]$.

Remarquons que si $\frac{a}{b}$ et $\frac{c}{d}$ sont deux rationnels de $\left[\frac{0}{1} ; \frac{1}{1}\right]$ sommets d'un même triangle de Farey, $i$ vérifie la formule suivante:

$$
i\left(\frac{a}{b} \oplus \frac{c}{d}\right)=\frac{i\left(\frac{a}{b}\right)+i\left(\frac{c}{d}\right)}{2}
$$

Le prolongement de $i$ se fait de la même façon sur les intervalles $\left[\frac{1}{1} ; \frac{1}{0}\right],\left[\frac{1}{0} ;-\frac{1}{1}\right]$ et $\left[-\frac{1}{1} ; \frac{0}{1}\right]$. On a obtenue une bijection $i: \widehat{\mathbb{Q}} \rightarrow \mathbb{Q}(2)$. On remarque que $i$ présèrve l'orientation du cercle. 


\section{I.1.d Le Bracelet de Ford}

Supposons un instant qu'un bracelet est une collection d'horocycles de $\mathbb{D}^{2}$ telle que deux horocycles voisins sont tangents. Nous donnerons la définition précise d'un bracelet dans la suite (définition I.3.1). On construit à présent, sans en donner les détails, une succession de bracelets $\left(b_{n}^{F}\right)_{n \in \mathbb{N}}$, chaque horocycle de $b_{n}^{F}$ étant basé en un point de $S_{n}$.

Soit $h_{i}^{F}$ l'horocycle basé en $u_{i}$ passant par le centre du disque de Poincaré, pour $i \in\{1 ; 2\}$. Alors il existe un unique horocycle $h_{3}^{F}$ tangent à $h_{1}^{F}$ et $h_{2}^{F}$, à gauche de l'arête orientée $\left(\frac{0}{1} ; \frac{1}{0}\right)$. Un petit argument de symétrie montre que $h_{3}^{F}$ est nécessairement basé en $u_{3}=-\frac{1}{1}$.

On note $b_{1}$ le bracelet $\left\{h_{1}, h_{2}, h_{3}\right\}$. On transporte alors ces horocycles par $P S L_{2}(\mathbb{Z})$ en remarquant que si $\gamma \in P S L_{2}(\mathbb{Z})$ permute les points $u_{1}, u_{2}$ et $u_{3}$, alors il permute aussi les horocycles associés. On obtient ainsi une collection d'horocycles $\left\{h_{n}^{F}\right\}_{n \in \mathbb{N}}$ telle que $h_{n}$ est basé en $u_{n} \in \widehat{\mathbb{Q}}$. Cette collection est notée $b^{F}$ et s'appelle le bracelet de Ford. La collection $b^{F}$ est invariante par $P S L_{2}(\mathbb{Z})$.
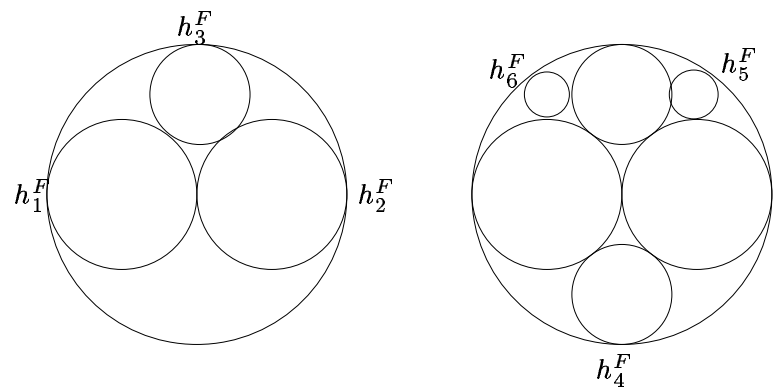

FIG. I.2 - Bracelet de Ford partiel.

On pose $b_{n}^{F}=\left\{h_{i}^{F}\right\}_{i=1,3 \cdot 2^{n-1}}$ et on obtient ainsi un bracelet basé en $S_{n}$ défini comme étant le bracelet de Ford d'ordre $n$.

Pour ne pas créer de confusion, précisons que l'ordre naturel sur $\widehat{\mathbb{Q}}$ ne sera pas utilisé pour le bracelet de Ford.

\section{I.1.e Deux topologies d'une limite inductive d'espaces métriques}

Nous donnons deux topologies naturelles sur une limite inductive d'espaces métriques. Ces espaces interviendront tout au long du texte.

Soit $\left(E_{n}, d_{n}\right)_{n \in \mathbb{N}}$ une suite strictement croissante d'espaces métriques (resp. topologiques). C'est à dire que pour chaque $n \in \mathbb{N}$, il existe une isométrie (resp. un plongement) $i_{n}: E_{n} \rightarrow E_{n+1}$. Ensemblistement, la limite inductive du système $\left(E_{n}, i_{n}\right)$, notée $E$ est l'union disjointe des espaces $E_{n}$ quotientée par la relation d'équivalence engendrée par les relations $i_{n}\left(x_{n}\right) \sim x_{n}$. Par exemple si $E_{n}$ est l'espace euclidien réel de 
dimension $n$, la limite inductive s'identifie à l'ensemble des suites réelles stationnaires en 0 . Il apparait alors deux topologies naturelles sur l'espace $E$.

La topologie faible: Elle se définit par les ouverts. Un sous-ensemble $U$ de $E$ est un ouvert si et seulement si pour tout $n$, l'ensemble $U_{n}=E_{n} \cap U$ est un ouvert de $E_{n}$. Muni de cette topologie, une suite $\left(u_{n}\right)_{n \geq 0}$ converge vers $u$ si et seulement si il existe $p \in \mathbb{N}$ tel que: $\forall n \in \mathbb{N}, u_{n} \in E_{p}, u \in E_{p}$ et $\left(u_{n}\right)_{n \geq 0}$ converge vers $u$ dans l'espace métrique (ou topologique) $E_{p}$. Avec cette topologie, si les espaces $E_{n}$ sont complets, il en est de même pour $E$. Notons que cette topologie n'est pas métrisable [Die-Sch]. Cette topologie sera utilisé dans le cadre plus générale où les applications $i_{n}$ sont des plongements (c.a.d. continues, ouvertes et injectives).

La topologie métrique: Soit $(x, y)$ un couple d'éléments de $E$ et $p$ un entier tel que ces deux éléments sont dans $E_{p}$. Puisque les $i_{n}$ sont des isométries, on a

$$
d_{n}(x ; y)=d_{p}(x ; y) \text { si } n \geq p
$$

Donc le nombre $d(x ; y)=d_{n}(x ; y)$ si $(x, y) \in E_{n}^{2}$ est bien défini et indépendant de $n$. Cela définit ainsi une métrique sur l'espace $E$.

Afin d'éclairer les topologies des espaces que nous étudions dans cette thèse, nous donnons ici un résultat de contractibilité valable pour les deux topologies. Soit $\mathbb{R}^{(\infty)}$ l'ensemble des suites réelles stationnaires égales à 0 . L'espace $\mathbb{R}^{(\infty)}$ hérite de la norme supérieure $\|x\|=\sup _{n \geq 0}\left|x_{n}\right|$. L'espace $\mathbb{R}^{(\infty)}$ est aussi la limite inductive de la suite:

$$
i_{n}:\left(x_{1}, \cdots, x_{n}\right) \in \mathbb{R}^{n} \mapsto\left(x_{1}, \cdots, x_{n}, 0\right) \in \mathbb{R}^{n+1}
$$

et peut être ainsi muni de la topologie faible. Les applications $i_{n}$ sont des isométries pour la norme supérieure. On a alors le résultat suivant:

Proposition I.1. L'espace $\mathbb{R}^{(\infty)}$ est contractile pour:

1. la topologie métrique provenant de la norme supérieure.

2. la topologie faible.

Preuve. - Ici, la topologie métrique provenant de la norme coïncide avec la topologie de la norme. On vérifie aisément que l'application $(x, t) \in \mathbb{R}^{(\infty)} \times[0 ; 1] \mapsto(1-t) x \in$ $\mathbb{R}^{(\infty)}$ est continue pour les deux topologies et fournit une rétraction par déformation de $\mathbb{R}^{(\infty)}$ sur l'origine.

En dimension finie, enlever un point à un espace topologique ajoute de l'homotopie. Ce n'est pas toujours vrai en dimension infinie, comme le montre la proposition suivante qui sera utile par la suite.

Proposition I.2. L'espace $\mathbb{R}^{(\infty)} \backslash\{0\}$ est contractile pour:

1. la topologie métrique provenant de la norme supérieure.

2. la topologie faible.

Preuve. - En effet, l'application

$f_{t}:\left(x_{1}, x_{2}, \cdots\right) \in \mathbb{R}^{(\infty)} \backslash\{0\} \mapsto(1-t)\left(x_{1}, x_{2}, \cdots\right)+t\left(0, x_{1}, x_{2}, \cdots\right) \in \mathbb{R}^{(\infty)} \backslash\{0\}$ 
fournit une homotopie entre l'identité et l'application $f\left(x_{1}, x_{2}, \cdots\right)=\left(0, x_{1}, x_{2}, \cdots\right)$. De même, l'application

$$
g_{t}:\left(x_{1}, x_{2}, \cdots\right) \in \mathbb{R}^{(\infty)} \backslash\{0\} \mapsto(1-t)\left(0, x_{1}, x_{2}, \cdots\right)+t(1,0,0, \cdots) \in \mathbb{R}^{(\infty)} \backslash\{0\}
$$

fournit une homotopie entre $f$ et l'application constante $g\left(x_{1}, x_{2}, \cdots\right)=(1,0,0, \cdots)$.

Ces applications sont continues pour la norme supérieure.

Pour la topologie faible, remarquons que la restriction de $f$ (resp. $g$ ) sur $\mathbb{R}^{n} \backslash\{0\} \times I$ est continue à valeurs dans $\mathbb{R}^{n+1} \backslash\{0\} \times I$. Ceci assure la continuité de $f$ et de $g$ pour la topologie faible.

On rappelle enfin un résultat découlant de la définition de la topologie faible.

Proposition I.3. Soit $\left(E_{n}\right)_{n>0}$ et $\left(F_{n}\right)_{n>0}$ deux suites strictement croissantes d'espaces métriques, telles que $E_{n}$ est homéomorphe à $F_{n}$ pour tout $n \in \mathbb{N}$. Alors leurs limites directes respectives $E$ et $F$, munies de la topologie faible sont homéomorphes.

Dans la suite de cette thèse, nous traiterons les problèmes dans le cas de la topologie faible. C'est également le cadre dans lequel P. Greenberg énonce ses résultats [Gre4]. Notons que la proposition I.3 est à priori fausse pour les topologies métriques, ce qui complique les questions de topologie dans ce cas. Nous reviendrons cependant sur les topologies métriques à la section II.4.

\section{I.2 La géométrie projective par morceaux}

Nous décrivons l'espace des homéomorphismes du cercle qui sont constitués d'un nombre fini de morceaux coïncidant avec des éléments de $P S L_{2} \mathbb{R}$. On demande de plus que ces homéomorphismes soient de classe $\mathcal{C}^{1}$. Cette condition supplémentaire a une interprétation géométrique naturelle (proposition I.4 et corollaire I.1). Cette géométrie CPP (abrégé en anglais de " ${ }^{1}$-piecewise projective) a été introduit par P.Greenberg dans [Gre2].

\section{I.2.a Définitions}

Définition I.2.1. (configuration ordonnée)

On appelle $\mathbf{n}$-configuration (cycliquement) ordonnée du cercle une suite $\left(x_{i}\right)_{i=1, n} \in\left(\mathbb{S}^{1}\right)^{n}$ vérifiant:

$$
\forall i \in<1, n>\exists \theta_{i} \in \mathbb{R} \mid x_{i}=e^{j \theta_{i}} \text { et } \theta_{1}<\theta_{2}<\ldots<\theta_{n}<\theta_{1}+2 \pi
$$

Définition I.2.2. (Homeomorphisme CPP)

Un homéomorphisme du cercle f est de classe $\mathcal{C}^{1}$ projectif par morceaux (ou CPP) s'il est de classe $\mathcal{C}^{1}$ et si il existe une configuration ordonnée $\left(x_{i}\right)_{i=1, n} \in\left(\mathbb{S}^{1}\right)^{n}$ telle que:

$$
\forall i \in<1 ; n>\exists h_{i} \in P S L_{2}(\mathbb{R}) \mid h_{i \mid\left[x_{i} ; x_{i+1}\right]}=f_{\mid\left[x_{i} ; x_{i+1}\right]}
$$

On note

$$
C P P=\{\text { homéomorphismes de classe CPP }\}
$$


On munit l'ensemble $C P P$ de la topologie compacte-ouverte $\mathcal{C}^{1}$ sur le cercle. Celleci est métrisable par la métrique suivante:

$$
d_{c p p}(f ; g)=\operatorname{Max}\left\{\sup _{x \in \mathbb{S}^{1}} d(f(x) ; g(x)) ; \sup _{x \in \mathbb{S}^{1}}\left|f^{\prime}(x)-g^{\prime}(x)\right|\right\} \forall(f, g) \in C P P^{2}
$$

Les germes: Pour tout $x \in \mathbb{S}^{1}$ et $f \in C P P$ on note $f_{x}^{l}$ (resp. $f_{x}^{r}$ ) le germe à gauche (resp. à droite) c'est à dire l'élément de $P S L_{2}(\mathbb{R})$ restriction de $f$ à gauche (resp. à droite) de $x$ pour l'orientation du cercle. Le germe d'un élément de $P S L_{2} \mathbb{R}$ s'identifie à cet élément puisque $P S L_{2} \mathbb{R}$ agit analytiquement sur $\mathbb{S}^{1}$.

Les points de coupure: Un point de coupure pour $f$ est un point de $\mathbb{S}^{1}$ où

$$
D_{x} f=\left(f_{x}^{r}\right)^{-1} \circ f_{x}^{l}
$$

n'est pas l'identité. L'élément $D_{x} f$ est appelé décalage de l'application $f$ en $x$. Il mesure le défaut d'être localement $P S L_{2} \mathbb{R}$ au voisinage de $x$. L'ensemble des points de coupure de $f$ est noté $b k(f)=\left(x_{i}\right)_{i=1, n}$ dans la mesure où l'on a ordonné les $x_{i}$. La notation " $b k$ " provient de l'anglais "breaks" ou "breakpoints".

Remarquons que $C P P$ est stable par composition et par passage à l'inverse. C' est donc un sous-groupe de $\mathrm{Hom} \mathrm{eo}^{+}\left(\mathbb{S}^{1}\right)$.

Par définition de $C P P$, on remarque que l'application $f \in C P P$ se prolonge sur $\mathbb{D}^{2}$ privé du polygone idéal $Q_{f}$ dont les sommets sont les points de coupures de $f$ (prolongement sur la partie hachurée dans la figure I.3), par l'isométrie $h_{i}$ sur chaque composante connexe de $\mathbb{D}^{2} \backslash Q_{f}$ bordé par l'intervalle correspondant à $h_{i}$.

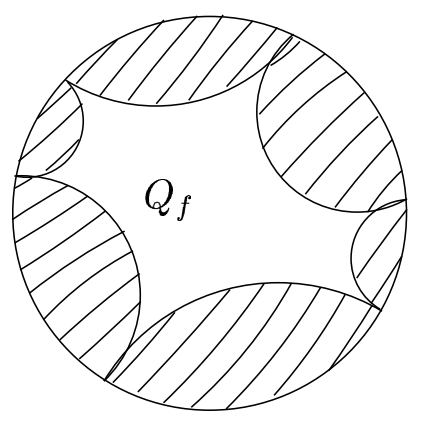

FIG. I.3 - Prolongement d'un élément de CPP.

Pourrait-on prolonger $f$ à $\mathbb{D}^{2}$, tout du moins à $\mathbb{D}^{2}$ privé d'un compact? Cela ne semble pas envisageable de manière immédiate, sans faire appel à la théorie des homéomorphismes quasiconformes (voir [Gar-Lak]). Cependant on peut faire agir $C P P$ sur l'ensemble des horocycles de $\mathbb{D}^{2}$, y compris ceux basés en les points de coupures des éléments de $C P P$.

Un horocycle est un cercle de $\mathbb{D}^{2}$ tangent à $\mathbb{S}^{1}=\partial \mathbb{D}^{2}$. On note

$$
\mathcal{H}=\left\{\text { horocycles de } \mathbb{D}^{2}\right\}
$$


muni de la métrique suivante:

$$
d_{\mathcal{H}}\left(h_{x} ; h_{y}\right)=\operatorname{Max}\left\{d_{\mathbb{S} 1}(x ; y) ;\left|d_{x}-d_{y}\right|\right\}
$$

où $d_{x}$ représente le rayon euclidien de $h_{x}$ pour le modèle du disque de Poincaré $\mathbb{D}^{2}$, vu comme disque unité du plan euclidien.

Par convention, le point-base d'un horocycle $h_{i}\left(\right.$ resp. $\left.h_{x}\right)$ sera noté $x_{i}$ (resp. $x$ ). On peut voir un horocycle $h_{x}$ comme orbite du sous-groupe des isométries paraboliques fixant $x$. La condition $\mathcal{C}^{1}$ dans la définition I.2.2 entraine la:

Proposition I.4. Si $f \in C P P$, alors l'élément $D_{x} f \in P S L_{2}(\mathbb{R})$ est un parabolique fixant $x$.

Preuve. - Puisque $f$ est de classe $\mathcal{C}^{0}$ en $x$, on a $f_{x}^{l}(x)=f_{x}^{r}(x)$. Ainsi, $D_{x} f$ fixe le point $x$. Par ailleurs, $f$ étant de classe $\mathcal{C}^{1}$ en $x, D_{x} f$ a pour dérivée 1 en $x$. C'est donc un élément parabolique de $P S L_{2} \mathbb{R}$ fixant $x$.

De plus, le sous-groupe des paraboliques fixant $x$, fixe ensemblistement n'importe quel horocycle $h_{x}$ basé en $x$. On en déduit l'action à gauche de CPP sur l'ensemble des horocycles de $\mathbb{D}^{2}$. Soit $x \in \mathbb{S}^{1}$ et $h_{x}$ basé en $x$, alors $D_{x} f\left(h_{x}\right)=h_{x}$, par conséquent on a l'égalité $f_{x}^{l}\left(h_{x}\right)=f_{x}^{r}\left(h_{x}\right)$.

Corollaire I.1. CPP agit à gauche sur $\mathcal{H}$ :

$$
\begin{array}{ccccc}
C P P & \times & \mathcal{H} & \longrightarrow & \mathcal{H} \\
(f & , & \left.h_{x}\right) & \longmapsto & f \cdot h_{x}=f_{x}^{l}\left(h_{x}\right)=f_{x}^{r}\left(h_{x}\right)
\end{array}
$$

C'est une sorte de donnée géométrique supplémentaire du comportement d'un élément de $C P P$ que l'on utilisera systématiquement dans la suite, essentiellement en des points de coupure des applications CPP. Il faut cependant souligner que cette action ne porte que sur les horocycles et non sur les éléments de ceux-ci. Autrement dit, on pensera un horocycle $h_{x}$ comme un point au dessus de $x$, et non comme une courbe plongée dans $\mathbb{D}^{2}$. On est maintenant en mesure de définir l'espace de Greenberg $\mathcal{G} r$. C'est l'espace central dans cette thèse.

Définition I.2.3. (L'espace $\mathcal{G} r$ )

Soit $\widehat{\mathcal{G r}}\left(C P P_{\mathbb{Q}}\right.$ dans l'introduction) l'espace suivant:

$$
\widehat{\mathcal{G} r}=\{f \in C P P \mid b k(f) \subset \widehat{\mathbb{Q}}\}
$$

On définit alors

$$
\mathcal{G} r=\left\{f \in \widehat{\mathcal{G} r} \mid f\left(\frac{0}{1}, \frac{1}{0},-\frac{1}{1}\right)=\left(\frac{0}{1}, \frac{1}{0},-\frac{1}{1}\right)\right\}
$$

On définit de manière analogue les espaces:

$$
\begin{gathered}
\widehat{\mathcal{G} r_{n}}=\left\{f \in \widehat{\mathcal{G} r} \mid b k(f) \subset S_{n}\right\} \\
\mathcal{G} r_{n}=\left\{f \in \widehat{\mathcal{G} r_{n}} \mid f\left(\frac{0}{1}, \frac{1}{0},-\frac{1}{1}\right)=\left(\frac{0}{1}, \frac{1}{0},-\frac{1}{1}\right)\right\}=\widehat{\mathcal{G} r_{n}} \cap \mathcal{G} r
\end{gathered}
$$


L'égalité:

$$
b k(h \circ f)=b k(f) \forall(f, h) \in C P P \times P S L_{2}(\mathbb{R})
$$

couplée à la 3-transitivité de $P S L_{2} \mathbb{R}$ sur le cercle, montre que $\mathcal{G} r$ est homéomorphe au quotient de $\widehat{\mathcal{G r}}$ par l'action de composition à gauche de $P S L_{2} \mathbb{R}$. Il en est de même pour $\mathcal{G} r_{n}$ relativement à $\widehat{\mathcal{G} r_{n}}$. Les espaces $\mathcal{G} r_{n}$ et $\widehat{\mathcal{G} r_{n}}$ seront munis de la topologie induite de la métrique $d_{C P P}$.

On remarque que $\mathcal{G} r$ (resp. $\widehat{\mathcal{G} r}$ ) est ensemblistement la limite inductive de la suite $\left(\mathcal{G} r_{n}\right)_{n \geq 0}\left(\operatorname{resp} .\left(\widehat{\mathcal{G} r_{n}}\right)_{n \geq 0}\right)$. Les inclusions

$$
\mathcal{G} r_{n} \stackrel{i_{n}}{\complement} \mathcal{G} r_{n+1}
$$

sont des isométries pour la métrique $d_{C P P}$. On munira $\mathcal{G} r$ et $\widehat{\mathcal{G} r}$ de la topologie faible.

D'autres normalisations de l'espace $P S L_{2} \mathbb{R} \backslash \widehat{\mathcal{G r}}$ seront utiles dans le texte, notamment dans les chapitres III et IV. Nous les expliciterons au moment ou nous en aurons besoin.

\section{I.2.b Hyperboliques généralisés-Déformations log-lambda}

Dans le groupe $P S L_{2} \mathbb{R}$, il y a trois types d'homéomorphismes, classifiés par la trace de leurs matrices associées: les éléments elliptiques dont la trace est dans l'intervalle ouvert ] $-2 ; 2$ [, les éléments paraboliques dont la trace est \pm 2 , et les éléments hyperboliques. Nous construisons ici, des exemples d'éléments de l'espace CPP qui généralisent les éléments hyperboliques. Ces exemples sont introduits dans [Gre4] (voir aussi [Pen2] pour le cas $n=2$ ).

Soit $x=\left(x_{i}\right)_{i=1,2 n} \in\left(\mathbb{S}^{1}\right)^{2 n}$ une configuration ordonnée du cercle ayant un nombre pair d'éléments et $t>0$. On définit l'application $h_{x}^{t}$ suivante:

Sur chaque intervalle de la forme $] x_{2 i} ; x_{2 i+1}\left[\right.$, on définit $h_{x}^{t}$ comme étant la restriction de l'élément hyperbolique d'axe la géodésique d'extrémités $x_{2 i}$ et $x_{2 i+1}$, et de dérivée $\frac{1}{t}$ en $x_{2 i}$ et $t$ en $x_{2 i+1}$. Sur les intervalles de la forme ] $x_{2 i+1} ; x_{2 i+2}$ [, on définit $h_{x}^{t}$ de manière analogue avec pour dérivée $t$ en $x_{2 i+1}$ et $\frac{1}{t}$ en $x_{2 i+2}$. Ces applications se recollent aux points $x_{i}, i \in<1 ; 2 n>$ pour donner ainsi un élément de $C P P$ ayant pour ensemble de points fixes et pour ensemble de points de coupure, la configuration ordonnée $x$.

Parmi ces exemples, le cas $n=2$ sera un objet utilisé dans bon nombre de démonstrations. Soit $Q$ un quadrilatère du disque de Poincaré de sommets $x=\left(x_{i}\right)_{i=1,4}$. Si $e$ désigne la diagonale $\left(x_{1} ; x_{3}\right)$, alors l'exemple précédent appliqué à la configuration $x$ sera appelée déformation log-lambda par rapport au couple $(Q, e)$ le long de $e$ et sera notée $\theta_{Q, e, t}$. Par ailleurs, on appelera déformation log-lambda modifiée le représentant normalisé de $\theta_{Q, e, t}$ dans $\mathcal{G} r$.

\section{I.3 Les espaces de bracelets}

On définit ici la notion de bracelets, également introduite par P. Greenberg [Gre3], qui 


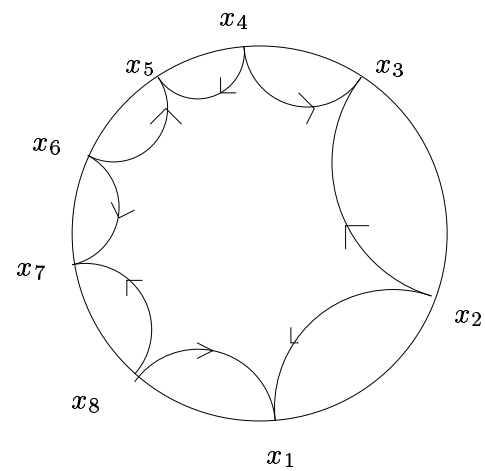

Hyperbolique généralisé $n=4$

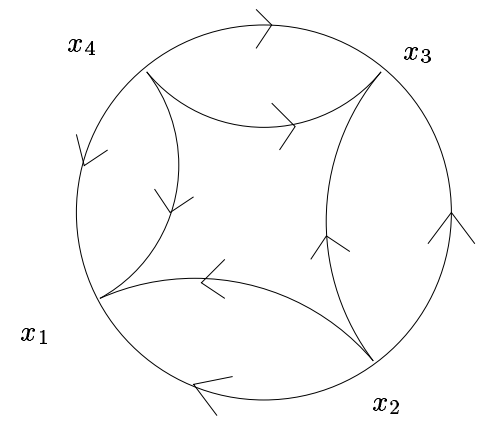

Déformation log-lambda

FIG. I.4 - Hyperboliques généralisés pour $n=4$ et $n=2$.

est en quelque sorte la version géométrique des applications de classe CPP (voir I.5.b).

\section{I.3.a Définition d'un bracelet}

Soit $(x, y) \in\left(\mathbb{S}^{1}\right)^{2}$ et $h_{x}$ (resp. $h_{y}$ ) un horocycle basé en $x$ (resp. $y$ ). Si $x \neq y$, on note $(x ; y)$ la géodésique d'extrémités $x$ et $y$ et $\hat{x}=h_{x} \cap(x ; y), \hat{y}=h_{y} \cap(x ; y)$. On définit alors $d_{h}\left(h_{x} ; h_{y}\right)= \pm d_{\mathbb{H}^{2}}(\hat{x} ; \hat{y})$ en convenant que cette valeur est positive si les deux horocycles ne s'intersectent pas et négative ou nulle sinon.

Si $x=y$, on peut encore définir le nombre $d_{h}\left(h_{x} ; h_{x}^{\prime}\right)$ comme étant la longueur du segment d'une géodésique tombant sur $x$, délimité par les intersections de cette géodésique avec les horocycles $h_{x}$ et $h_{x}^{\prime}$. On remarque sur la figure I.5 que cette longueur ne dépend aucunement du $z$ choisi.

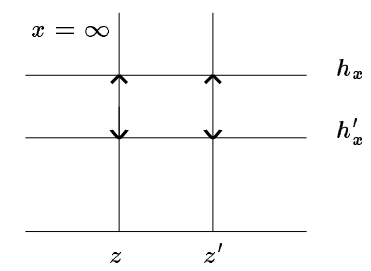

FIG. I.5 - Distance de deux horocycles.

La quantité $d_{h}\left(h_{x} ; h_{y}\right)$ n'est évidemment pas une distance mais elle apparaitra naturellement dans la partie II.

Définition I.3.1. (Bracelet)

Un n-bracelet est la donnée d'une configuration ordonnée du cercle $\left(x_{i}\right)_{i=1, n}$ et d'une collection d'horocycles $\left\{h_{i}\right\}_{i=1, n}$ où $h_{i}$ est basé en $x_{i}$ telles que:

$$
\forall i \in<1, n>, d_{h}\left(h_{i} ; h_{i+1}\right)=0 \text { et } d_{h}\left(h_{n} ; h_{1}\right)=0
$$


Un n-bracelet propre est un n-bracelet qui ne contient pas de couple d'horocycles s'intersectant transversalement.

Dans la suite du texte, on notera un bracelet $b=\left(h_{i}\right)_{i \in<1 ; n>}$, étant entendu que ses points-base forment une configuration ordonnée du cercle $\left(x_{i}\right)_{i \in<1 ; n>}$. Celle-ci sera notée $b k(b)$.

La condition $d_{h}\left(h_{i} ; h_{i+1}\right)=0$ signifie exactement que les horocycles $h_{i}$ et $h_{i+1}$ sont tangents. La figure I.6 montre deux exemples de bracelets. Le premier bracelet est propre, le deuxième ne l'est pas.
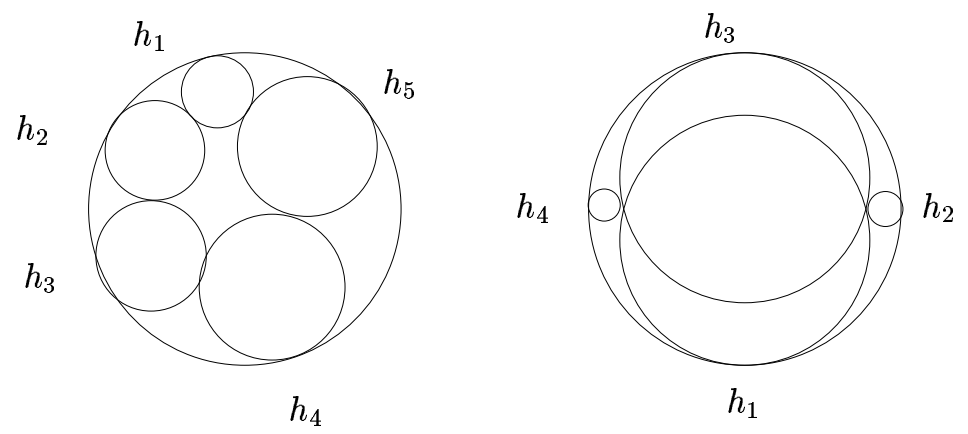

FIG. I.6 - Exemples de bracelets.

On voudrait définir un espace de bracelets et le munir d'une topologie. Il semble naturel de dire que deux bracelets $b=\left(h_{1}, \ldots, h_{n}\right)$ et $b^{\prime}=\left(h_{1}^{\prime}, \ldots, h_{p}^{\prime}\right)$ sont proches si et seulement si pour tout $i, h_{i}$ est proche de $h_{i}^{\prime}$. Cela suppose que $n=p$. Pour comparer deux bracelets, il faut qu'ils aient a priori le même nombre d'horocycles. On va utiliser des espaces filtrés pour définir un espace de bracelet "contenant" tous les bracelets.

\section{I.3.b Filtrations des espaces de bracelets}

Définition I.3.2. (Espaces de bracelets)

On définit les espaces suivants:

$$
\widehat{\mathcal{C}_{n}}=\{(n+1)-\text { bracelets }\} \text { et } \widehat{\mathcal{B}_{n}}=\{(n+1)-\text { bracelets propres }\}
$$

puis:

$$
\widehat{\mathcal{C} r_{n}}=\left\{3 \cdot 2^{n-1}-\text { bracelets }\right\} \text { et } \widehat{\mathcal{B} r_{n}}=\left\{3 \cdot 2^{n-1}-\text { bracelets propres }\right\}
$$

et leurs normalisés par $P S L_{2} \mathbb{R}$ :

$$
\begin{gathered}
\mathcal{C}_{n}=\left\{\left(h_{1} ; \cdots ; h_{n+1}\right) \in \widehat{\mathcal{C}_{n}} \mid h_{1}=h_{0}^{F}, \quad h_{n+1}=h_{\infty}^{F}\right\} \\
\mathcal{C} r_{n}=\left\{b=\left(h_{x_{i}}\right)_{i=1,3 \cdot 2^{n-1}} \in \widehat{\mathcal{C} r_{n}} \mid x_{1}=\frac{0}{1}, x_{2^{n-1}+1}=\frac{1}{0}, x_{3 \cdot 2^{n-1}}=-\frac{1}{1}\right\}
\end{gathered}
$$

Les normalisés $\widehat{\mathcal{B}_{n}}$ et $\widehat{\mathcal{B} r_{n}}$ sont définis de la même manière. 
Remarquons au passage que le bracelet de Ford $b_{n}^{F}$ est un élément de $\mathcal{B} r_{n}$. On introduit maintenant des limites directes des espaces $\left(\widehat{\mathcal{C} r_{n}}\right)_{n \geq 1}$ et $\left(\mathcal{C} r_{n}\right)_{n \geq 1}$. Pour cela, on utilise une propriété d'unicité portant sur les triplets d'horocycles.

Proposition I.5. Soit $h_{1}, h_{3}$ un couple d'horocycles tangents. Alors, il existe exactement un horocycle $h_{2}$ à droite de l'arête orientée $\left(x_{1} ; x_{3}\right)$, tangent à $h_{1}$ et $h_{3}$.

Preuve. - Partons du point base $x_{1}$ de $h_{1}$. En chaque point $x \in \mathbb{S}^{1}$, il y a un unique horocyle $h_{x}$ basé en ce point et tangent à $h_{1}$. Lorsque l'on se déplace le long de cette famille d'horocycles en faisant varier $x$ de $x_{1}$ à $x_{2}$ à droite de l'arête orientée $\left(x_{1} ; x_{3}\right)$, l'horocycle $h_{x}$ grandit (son rayon euclidien augmente) jusqu'à ce qu'il rencontre l'horocyle $h_{3}$. Il est alors tangent à $h_{3}$. Continuons de nous déplacer vers $x_{3}$, alors $h_{x}$ devient transversale à $h_{3}$.

Cette proposition permet de définir une inclusion naturelle

$$
i_{n}: \widehat{\mathcal{C} r_{n}} \rightarrow \widehat{\mathcal{C} r_{n+1}}, n \geq 1
$$

comme suit. Grâce à la proposition I.5, $\forall i \in<1,3 \cdot 2^{n-1}>$ il existe un unique horocycle $h_{i}^{\prime}$ tangent à $h_{i}$ et $h_{i+1}\left(h_{3 \cdot 2^{n-1}+1}=h_{1}\right.$ par convention $)$ tel que les points-base $x_{i}, x_{i}^{\prime}$ et $x_{i+1}$ forment une configuration ordonnée de $\mathbb{S}^{1}$. En particulier, l'horocycle $h_{i}^{\prime}$ est dans le demi-disque fermé délimité par la géodésique $\left(x_{i} ; x_{i+1}\right)$ ne contenant pas les points-base $\left\{x_{j}\right\}_{j \notin\{i ; i+1\}}$.

La collection

$$
i_{n}(b)=\left(h_{1}, h_{1}^{\prime}, \cdots, h_{i}, h_{i}^{\prime}, h_{i+1}, \cdots, h_{3 \cdot 2^{n-1}-1}^{\prime}, h_{3 \cdot 2^{n-1}}, h_{3 \cdot 2^{n-1}}^{\prime}\right)
$$

est un élément de $\widehat{\mathcal{C} r_{n+1}}$. L'application $i_{n}$ ainsi définie est compatible avec la normalisation introduite plus haut. Elle passe donc aux normalisés en une application, évidemment injective, $i_{n}: \mathcal{C} r_{n} \rightarrow \mathcal{C} r_{n+1}$. On a ainsi construit deux systèmes inductifs et les limites sont notées:

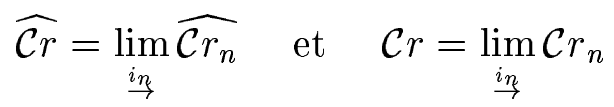

Concernant les bracelets propres, notons que $i_{n}\left(\widehat{\mathcal{B} r_{n}}\right) \subset \widehat{\mathcal{B} r_{n+1}}$. En effet, si $h_{i}$ et $h_{i+1}$ sont deux horocycles voisins d'un bracelet $b \in \widehat{\mathcal{B} r_{n}}$, alors l'horocycle ajouté $h_{i}^{\prime}$ est tangent à $h_{i}$ et à $h_{i+1}$. Il est de plus contenu dans le demi-disque délimité par la géodésique $\left(x_{i} ; x_{i+1}\right)$ ne contenant que les points-base $x_{i}$ et $x_{i+1}$. Puisque $b$ est propre, ce demi-disque n'intersecte aucun horocycle de $b$ autre que $h_{i}$ et $h_{i+1}$. Ceci montre que $i_{n}(b)$ est propre. La filtration précédente se restreint donc aux bracelets propres. On définit donc:

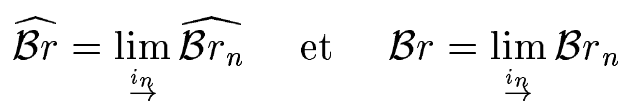




\section{I.3.c Topologie des espaces de bracelets}

Soit $n \in \mathbb{N}^{*}$. L'espace des bracelets $\widehat{\mathcal{C} r_{n}}$ peut être vu comme sous-ensemble de l'espace produit $\mathcal{H}^{3 \cdot 2^{n-1}}=\mathcal{H}^{S_{n}}$, puisque $\operatorname{card}\left(S_{n}\right)=3 \cdot 2^{n-1}$. On le munit de la trace de la topologie produit sur $\mathcal{H}^{3 \cdot 2^{n-1}}$. Les inclusions

$$
i_{n}: \widehat{\mathcal{C} r_{n}} \rightarrow \widehat{\mathcal{C} r_{n+1}}
$$

sont des plongements. Les espaces $\widehat{\mathcal{B} r}, \widehat{\mathcal{C} r}, \mathcal{B} r$ et $\mathcal{C} r$ sont ainsi munis de la topologie faible.

\section{I.4 L'espace de Teichmüller universel}

Cette section fournit une introduction des objets "universels" définis dans [Pen2].

Soit $F_{g}^{s}=F$ une surface de genre $g$ avec $s$ points marqués. On suppose que la caractéristique d'Euler de la surface $\chi(F)=2-2 g-s$ est négative. On note $\mathcal{T}_{g}^{s}$ l'espace de Teichmüller de la surface. Dans [Pen1], l'auteur définit $\widetilde{\mathcal{T}_{g}^{s}}$, espace de Teichmüller décoré de $F$. Il s'agit d'un $\mathbb{R}_{+}^{s}$-fibré sur $\mathcal{T}_{g}^{s}$, dont la fibre correspond aux collections d'horocycles en les points marqués de la surface $F$. L'espace $\widetilde{\mathcal{T}_{g}^{s}}$ peut se voir comme l'ensemble des tesselations décorées de $\mathbb{D}^{2}$ invariantes par le groupe fondamental de la surface.

A la recherche d'analogues universels, R.Penner considère l'espace de Teichmüller universel (resp. décoré) Tess (resp. Tess). Celui-ci est universel au sens qu'il contient tous les espaces de Teichmüller (resp. décorés) $\widetilde{\mathcal{T}_{g}^{s}}$. C'est un modèle de nature topologique. Il contient strictement l'espace de Teichmüller universel de Bers (plus classique):

$$
T(1)=P S L_{2} \mathbb{R} \backslash\left\{\text { homéomorphismes quasi-symétriques de } \mathbb{D}^{2}\right\}=P S L_{2} \mathbb{R} \backslash Q S
$$

\section{I.4.a Tesselations et tesselations décorées}

Définition I.4.1. (Tesselation)

Une tesselation $\tau^{\prime}$ est une collection dénombrable et localement finie de géodésiques, appelées arêtes de la tesselation, formant une triangulation idéale de $\mathbb{D}^{2}$. Une tesselation $\tau$ est dite marquée si elle est munie d'une arête orientée distinguée $e \in \tau$. On notera alors:

$$
\text { Tess }^{\prime}=\{(\tau, e) \text { tesselations marquées }\}
$$

Dorénavant, une tesselation sera toujours munie d'une arête orientée distinguée. On oubliera donc le mot "marquée". Si il n'y a pas de marquage, on parlera plutôt de triangulation.

Un exemple de tesselation est la triangulation de Farey munie de l'arête orientée $\left(\frac{0}{1} ; \frac{1}{0}\right)$. On la note $\tau_{*}$. Elle joue le rôle de point-base de l'espace topologique Tess'. 
Définition I.4.2. (Tesselation décorée)

Une tesselation décorée $\tilde{\tau}$ est la donnée d'une tesselation (marquée) $\tau$ et d'un horocycle en chaque sommet de $\tau$ tels que la famille d'horocycles est localement finie dans $\mathbb{D}^{2}$. L'ensemble des tesselations décorées est noté $\widetilde{T e s s}$.

Puisque $\tau_{*}^{(0)}=\widehat{\mathbb{Q}}$, on peut décorer la tesselation de Farey par le bracelet de Ford $b^{F}$ introduit au paragraphe I.1.d. On obtient ainsi une tesselation décorée $\tilde{\tau}_{*}$, appelée tesselation décorée de Farey-Ford. Elle joue le rôle de point base de l'espace $\widetilde{\text { Tess }}$.

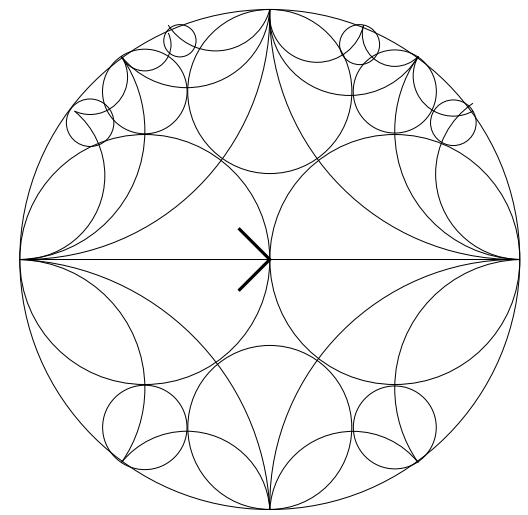

FIG. I.7 - Tesselation décorée de Farey-Ford.

On notera $\tau^{(0)} \subset \mathbb{S}^{1}$ l'ensemble des extrémités des éléments de $\tau$ (dense dans $\mathbb{S}^{1}$ par définition) et $\tau^{(2)}$ l'ensemble des triangles complémentaires de $\tau$ dans $\mathbb{D}^{2}$. Distinguer une arête orientée de $\tau$ revient également à distinguer une configuration ordonnée $x(\tau)=\left(x_{i}\right)_{i=1,3}$ qui correspond aux sommets d'un unique triangle $T \in \tau^{(2)}$, tel que $\left(x_{1} ; x_{2}\right)$ soit l'arête orientée distinguée de $\tau$. Le groupe $P S L_{2} \mathbb{R}$ agit à gauche par isométries sur Tess' et $\widetilde{T e s s}$. On prendra la normalisation suivante:

$$
\text { Tess }=\left\{\tau^{\prime} \in \text { Tess }^{\prime} \mid x(\tau)=\left(\frac{0}{1}, \frac{1}{0},-\frac{1}{1}\right)\right\}
$$

La même normalisation est adoptée pour l'espace $\widetilde{\text { Tess. }}$ L'espace Tess (resp. $\widetilde{\text { Tess }}$ ) est appelé l'espace de Teichmüller universel de Penner (resp. espace de Teichmüller universel décoré. On rappelle sans démonstration des propriétés combinatoires des tesselations. 


\section{I.4.b Rigidité combinatoire des tesselations}

Soit $\left(\tau_{i}\right)_{i \in<1,2>}$ un couple de tesselations distinguées par les triplets $\left(x_{i}, y_{i}, z_{i}\right)_{i=1,2}$, alors les trois propriétés suivantes sont vérifiées:

1. Il existe une unique bijection:

$$
\begin{aligned}
& f^{(0)}: \quad \tau_{1}^{(0)} \quad \longrightarrow \quad \tau_{2}^{(0)} \\
& \left(x_{1}, y_{1}, z_{1}\right) \quad \longmapsto \quad\left(x_{2}, y_{2}, z_{2}\right) \\
& (x, y, z) \in \tau_{1}^{(2)} \longmapsto f^{(0)} \cdot(x, y, z) \in \tau_{2}^{(2)}
\end{aligned}
$$

2. $f^{(0)}$ induit une bijection:

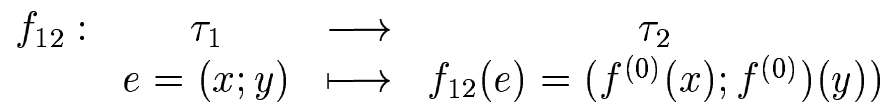

3. Les ensembles $\mathbb{Q}^{\prime}$ et $\tau$ sont en bijection.

Pour se convaincre de ces propriétés, il suffit de se rappeler de la combinatoire mise en jeu dans la construction d'une triangulation à partir d'un triangle $T_{0}$ (voir I.1.b), en commençant par trianguler le demi-disque se trouvant à gauche de l'arête orientée distinguée.

\section{I.4.c Topologies de Tess et de $\widetilde{\text { Tess }}$}

Reprenons la tesselation de Farey $\tau_{*}$. Les propriétés combinatoires précédentes montrent que pour toute tesselation $\tau$, il existe une unique bijection entre $\tau_{*}^{(0)}$ et $\tau^{(0)}$ qui fournit une application $f_{\tau}^{(0)}: \tau_{*}^{(0)} \rightarrow \mathbb{S}^{1}$. L'application $\tau \mapsto f_{\tau}^{(0)}$ ainsi construite fournit un plongement de $T e s s^{\prime}$ dans $\left(\mathbb{S}^{1}\right)^{\tau_{*}^{(0)}}$ muni de la topologie compacte-ouverte. Notons que la topologie compacte-ouverte s'identifie à la topologie produit lorsque $\tau_{*}^{(0)}$ est muni de la topologie discrète. On prend la topologie induite pour Tess'.

Pour la topologie de $\widetilde{\text {Tess}^{\prime}}$, le même raisonnement permet de plonger $\widetilde{\text {Tess}^{\prime}}$ dans l'espace produit $\mathcal{H}^{\tau_{*}^{(0)}}$ muni de la topologie produit.

Puisque $\tau_{*}^{(0)}=\widehat{\mathbb{Q}}$ est dense dans $\mathbb{S}^{1}$, l'application $f_{\tau}^{(0)}$ se prolonge en un homéomorphisme du cercle préservant l'orientation. On peut alors formuler le théorème suivant dû à Penner.

Théorème I.1 (Penner [Pen2]). Il existe un homéomorphisme $P S L_{2}(\mathbb{R})$-équivariant entre $\mathrm{Homeo}^{+}\left(\mathbb{S}^{1}\right)$ et Tess' qui donne au quotient un homéomorphisme entre PSL $(\mathbb{R}) \backslash$ Homeo $^{+}\left(\mathbb{S}^{1}\right)$ et Tess. 


\section{I.5 Liens entre les différents contextes géométriques}

On relie ici les homéomorphismes de classe CPP à l'espace de Teichmüller universel décoré. Plus précisément, l'espace $P S L_{2} \mathbb{R} \backslash C P P$, muni de la topologie $\mathcal{C}^{1}$ s'injecte continument dans l'espace $\widetilde{\text { Tess }}$ (proposition I.6). On exhibe ensuite un homéomorphisme entre l'espace des bracelets $\mathcal{C} r$ et l'espace $\mathcal{G} r$.

\section{I.5.a Une injection continue naturelle de $\mathcal{G} r$ dans $\widetilde{\text { Tess }}$}

Remarquons d'abord que $C P P$ est un sous-espace de $\mathrm{Homeo}^{+}\left(\mathbb{S}^{1}\right)$. Par conséquent, on peut plonger $P S L_{2}(\mathbb{R}) \backslash C P P$ dans Tess grâce au théorème I.1 pour la topologie $\mathcal{C}^{0}$ sur l'espace $C P P$.

Notons $\Psi$ la projection qui consiste à oublier les décorations. On cherche une application $s$ tel que le diagramme suivant soit commutatif:

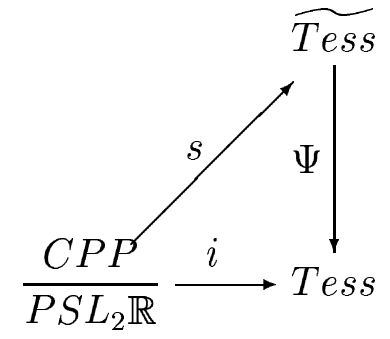

Proposition I.6. Il existe une injection continue $s$ de $P S L_{2}(\mathbb{R}) \backslash C P P$ pour la topologie de la métrique $d_{C P P}$ dans $\widetilde{T e s s}$ qui est une section de $\Psi$ au dessus de $P S L_{2}(\mathbb{R}) \backslash C P P$.

Preuve. - Soit $g \in P S L_{2}(\mathbb{R}) \backslash C P P$, c'est à dire que $g$ fixe point par point la configuration $\left(\frac{0}{1}, \frac{1}{0},-\frac{1}{1}\right)$. On définit $g \cdot \tilde{\tau}_{*}$ comme suit. Soit $e \in \tau_{*}$ dont les extrémités sont $x$ et $y$. On note $g(e)$ la géodésique d'extrémités $g(x)$ et $g(y)$. D'après [Pen2], la collection $\{g(e)\}_{e \in \tau_{*}}$ forme une tesselation qui est dans Tess car $g$ est normalisée. Cette collection donne la tesselation notée $g \cdot \tau_{*}$ (c'est l'image de $g$ par l'homéomorphisme réalisé dans le théorème I.1).

D'autre part, grâce à l'action de $C P P$ sur les horocycles, on peut définir sur $g \cdot \tau_{*}$ la décoration suivante:

$$
\forall z=g(x) \in\left(g \cdot \tau_{*}\right)^{(0)} \text {, où } x \in \tau_{*}^{(0)} \text {, on pose } h_{z}^{\prime}=g\left(h_{x}^{F}\right)
$$

où $h_{x}^{F}$ est l'horocycle basé en $x$ du bracelet de Ford. La tesselation $g \cdot \tau_{*}$ munie de cette décoration fournit un élément $g \cdot \tilde{\tau}_{*} \in \widetilde{\text { Tess. }}$.

On a ainsi défini une application $s: P S L_{2}(\mathbb{R}) \backslash C P P \rightarrow \widetilde{\text { Tess }}$ qui, par construction, est une section de $\Psi$ puisque $g \cdot \tau_{*}$ représente la tesselation décorée $g \cdot \tilde{\tau}_{*}$ à laquelle on enlève la décoration.

Il reste à montrer que $s$ est continue. D'après le lemme A.2. de [Mal-Pen], l'horocycle $f \cdot h_{x}^{F}$ est exactement l'horocycle de diamètre euclidien $\left|f^{\prime}(x)\right| d_{x}$ dans le modèle 
de $\mathbb{H}^{2}$, où $d_{x}$ est le diamètre de l'horocycle de Ford $h_{x}^{F}$ basé en $x \neq \infty$. Lorsque $x=\infty$, $f \cdot h_{\infty}^{F}$ est l'horocycle en $\infty$ ( $f$ est normalisé) de hauteur $\frac{1}{\left|f^{\prime}(\infty)\right|}$.

Par conséquent, pour tout $x \in \widehat{\mathbb{Q}}$, l'application

$$
s_{x}: f \in P S L_{2} \mathbb{R} \backslash C P P \rightarrow f \cdot h_{x} \in \mathcal{H}
$$

est continue. On en déduit que l'application $s$ est continue également.

Puisque $\mathcal{G} r$ est un sous-espace métrique de l'espace $C P P$, on obtient ainsi le:

Corollaire I.2. L'espace $\mathcal{G} r$ s'injecte continument dans $\widetilde{T e s s}$ par s.

\section{I.5.b Un homéomorphisme entre $\mathcal{C} r$ et $\mathcal{G} r$}

On construit dans cette section un homéomorphisme, dû à Greenberg [Gre4], entre $\mathcal{C} r$ et $\mathcal{G} r$, pour la topologie faible. Commençons par un lemme décrivant une action partielle de $C P P$ sur l'ensemble des bracelets.

Lemme 1.1. Si $f \in C P P$ et $b=\left(h_{i}\right)_{i=1, n}$ est un bracelet tels que bk $(f) \subset\left(x_{i}\right)_{i=1, n}$, Alors la collection $f \cdot b=\left(f\left(h_{i}\right)\right)_{i=1, n}$ est encore un bracelet.

Preuve. - Il suffit de montrer que $d\left(h_{i}, h_{i+1}\right)=d\left(f \cdot h_{i}, f \cdot h_{i+1}\right) \forall i \in \mathbb{Z} / n \mathbb{Z}$, puisque le premier terme est nul. Comme il n'y a pas de point de coupure entre $x_{i}$ et $x_{i+1}$, on a l'égalité des germes $f_{x_{i}}^{r}=f_{x_{i+1}}^{l}=f_{i}$ où $f_{i}$ est une isométrie. Par conséquent, on a $d\left(h_{i}, h_{i+1}\right)=d\left(f_{x_{i}}^{r}\left(h_{i}\right), f_{x_{i+1}}^{l}\left(h_{i+1}\right)\right)=d\left(f \cdot h_{i}, f \cdot h_{i+1}\right)$.

Le lien entre les bracelets et les applications de classe CPP apparait clairement dans la proposition suivante.

Proposition I.7. Soient $b=\left(h_{x_{i}}\right)_{i=1, n}$ et $b^{\prime}=\left(h_{y_{i}}^{\prime}\right)_{i=1, n}$ deux bracelets. Alors il existe un unique $f \in C P P$ tel que:

$$
b k(f) \subset b k(b) \text { et } f \cdot b=b^{\prime} .
$$

Preuve. - Par triple transitivité de l'action de $P S L_{2} \mathbb{R}$ sur le cercle, pour tout $i \in \mathbb{Z} / n \mathbb{Z}$, il existe un unique $f_{i} \in P S L_{2} \mathbb{R}$ vérifiant les égalités:

$$
f_{i}\left(x_{i}\right)=y_{i}, f_{i}\left(x_{i+1}\right)=y_{i+1} \text { et } f_{i}\left(h_{x_{i}}\right)=h_{y_{i}}^{\prime}
$$

On définit $f$ par morceaux de sorte que $f_{\mid\left[x_{i} ; x_{i+1}\right]}=\left(f_{i}\right)_{\mid\left[x_{i} ; x_{i+1}\right]}$ pour tout $i \in \mathbb{Z} / n \mathbb{Z}$. L'application $f$ est bien définie et continue puisque $f_{i}\left(x_{i+1}\right)=y_{i+1}^{\prime}=f_{i+1}\left(x_{i+1}\right)$. Elle transforme bijectivement chaque intervalle $\left[x_{i} ; x_{i+1}\right]$ en l'intervalle $\left[y_{i} ; y_{i+1}\right]$. C'est donc un homéomorphisme du cercle projectif par morceaux.

Puisque $f_{i}$ est un élément de $P S L_{2} \mathbb{R}$ agissant sur l'intervalle $\left[x_{i} ; x_{i+1}\right]$ et que les horocycles basés en ces points sont tangents, les horocycles images sont aussi tangents. Par conséquent, les horocycles $h_{y_{i+1}}^{\prime}$ et $f_{i}\left(h_{x_{i+1}}\right)$ sont deux horocycles tangents à $h_{y_{i}}^{\prime}$ et 
basés au même point $y_{i+1}$. Ils sont nécessairement égaux. Ceci montre en particulier que le décalage $D_{x_{i+1}} f=f_{i+1}^{-1} \circ f_{i}$ est un élément parabolique fixant $x_{i}$. On en déduit que $f$ est de classe $\mathcal{C}^{1}$ en $x_{i+1}$ pour tout $i \in \mathbb{Z} / n \mathbb{Z}$.

Finalement, l'application $f$ est un élément de $C P P$ uniquement déterminé par la condition $f \cdot b=b^{\prime}$, et par construction $b k(f) \subset b k(b)$, ce qui montre la proposition.

Ce résultat doit etre pensé comme une sorte de simple transitivité de l'action de $C P P$ sur les bracelets.

Corollaire I.3. L'application:

$$
\begin{aligned}
\psi_{n}: \mathcal{G} r_{n} & \longrightarrow \mathcal{C} r_{n} \\
f & \longmapsto f \cdot b_{n}^{F}
\end{aligned}
$$

réalise un homéomorphisme entre $\mathcal{G} r_{n}$ et $\mathcal{C} r_{n}$. Cet homéomorphisme se prolonge en un homéomorphisme $\psi$ entre $\mathcal{G} r$ et $\mathcal{C} r$ pour la topologie faible.

Preuve. - La bijectivité de $\psi_{n}$ provient directement de la proposition I.7. L'application $\psi_{n}$ est continue puisque $f \in \mathcal{G} r_{n} \mapsto f \cdot h_{x}^{F} \in \mathcal{H}$ est continue pour tout $x \in S_{n}$. Il reste à montrer la continuité de $\psi_{n}^{-1}$. Puisque les espaces $\mathcal{G} r_{n}$ et $\mathcal{C} r_{n}$ sont métrisables, il suffit de montrer que pour toute suite $\left(f_{p}\right)_{p \in \mathbb{N}}$ de $\mathcal{G} r_{n}$ et pour tout $f \in \mathcal{G} r_{n}$ telles que $\psi_{n}\left(f_{p}\right)$ converge vers $\psi_{n}(f)$, alors $f_{p}$ converge vers $f$ dans $\mathcal{G} r_{n}$.

Supposons donc que la suite de bracelets $\left(f_{p} \cdot b_{n}^{F}\right)_{p \geq 0}$ converge vers le bracelet $f \cdot b_{n}^{F}$. Soient $x$ et $y$ deux points consécutifs de $S_{n}$. L'intervalle $[x ; y]$ contient un unique $z \in S_{n+1} \backslash S_{n}$. Puisque les applications $f_{p}$ et $f$ sont $P S L_{2} \mathbb{R}$ sur l'intervalle $[x ; y]$, la proposition I.5 entraine que la suite d'horocycles $\left(f_{p} \cdot h_{z}^{F}\right)_{p \geq 0}$ converge vers l'horocycle $f \cdot h_{z}^{F}$, où $h_{z}^{F}$ est l'horocycle de Ford basé en $z$. En particulier la suite de fonction $\left(f_{p}\right)_{p \in \mathbb{N}}$ converge vers $f$ sur le triplet $\{x ; y ; z\}$. Notons $A_{p}$ (resp. $\left.A\right)$ la matrice de $\left(f_{p}\right)_{\mid[x ; y]}$ (resp. $\left.f_{\mid[x ; y]}\right)$. La proposition 4.5.4. dans [Bea] montre que la suite de matrices $A_{p}$ converge vers la matrice $A$.

Ceci entraine que la suite $f_{p}$ converge pour la métrique $d_{C P P}$ vers $f$ sur l'intervalle $[x ; y]$. Ceci permet de conclure que $\psi_{n}^{-1}$ est continue.

La proposition I.3 entraine directement que $\psi$ est un homéomorphisme pour la topologie faible.

\section{I.6 Le groupe de Thompson}

\section{I.6.a Historique}

Le groupe $P L_{2}\left(\mathbb{S}^{1}\right)$ est défini par Richard Thompson en 1965. C'est un des premiers exemple de groupe infini, simple, de présentation fini [Tho]. Voici la définition originale de ce groupe. Elle interviendra notamment dans la section IV.6.b. 
Définition I.6.1. (Groupe de Thompson: version PL)

Le groupe $P L_{2}\left(\mathbb{S}^{1}\right)$ est l'ensemble des homéomorphismes $f$ de $\mathbb{R} / \mathbb{Z}$ vérifiant les propriétés suivantes:

(1) $f$ est affine par morceaux, avec un nombre fini de morceaux dans $\mathbb{R} / \mathbb{Z}$.

(2) Les points de discontinuité de $f$ sont dyadiques (c.a.d. de la forme $\frac{a}{2^{b}}$ où $a$ et $b$ sont des entiers relatifs).

(3) Les dérivées à gauche et à droite sont des puissances de 2 en tout point.

(4) $f(0)$ est un nombre dyadique.

On définit également les sous-groupes de $P L_{2}\left(\mathbb{S}^{1}\right)$ suivants:

$$
\begin{gathered}
F=\left\{\gamma \in P L_{2}\left(\mathbb{S}^{1}\right) \mid \gamma(0)=0\right\} \\
F^{\prime}=\left\{\gamma \in P L_{2}\left(\mathbb{S}^{1}\right) \mid \text { le germe de } \gamma \text { en } 0 \text { est trivial }\right\}
\end{gathered}
$$

L'ensemble $P L_{2}\left(\mathbb{S}^{1}\right)$ est un groupe muni de la loi de composition des applications. Il s'agit là d'une définition de type géométrique. La notation $F^{\prime}$ n'est pas fortuite, puisque le groupe ainsi défini est aussi le sous-groupe dérivé de $F$. Ce groupe apparait alors dans divers domaines des mathématiques comme en théorie de l'homotopie ([Dyd] en 1977), en homologie des groupes ([Bro-Geo] en 1984), dans les systèmes dynamiques et en cohomologie des groupes ([Ghy-Ser] en 1987, [Bri] en 1997). Enfin P. Greenberg a commencé une étude plus géométrique ([Gre4] en 1992) du groupe $P L_{2}\left(\mathbb{S}^{1}\right)$ à l'aide des homéomorphismes projectifs par morceaux. Pour un historique complet des différents groupes de Thompson, on pourra se référer à [Bri] et [Can-Flo-Par]. Dans cette thèse, on reprend l'étude géométrique du groupe de Thompson commencée par P. Greenberg. Il s'agit donc de penser le groupe $P L_{2}\left(\mathbb{S}^{1}\right)$ comme un groupe discret agissant "convenablement" sur des espaces toplogiques recélant si possible des structures invariantes par le groupe. Deux définitions du groupe de Thompson vont être particulièrement utilisées dans la suite, l'une de type géométrique, et l'autre de type combinatoire. Nous explicitons maintenant ces deux définitions.

\section{I.6.b Définitions}

\section{Le groupe de Thompson $P P S L_{2} \mathbb{Z}$}

Voici à présent la définition du groupe de Thompson $T$ en contexte projectif par morceaux.

Définition I.6.2. (Groupe de Thompson: version CPP)

Le groupe $P P S L_{2} \mathbb{Z}$ est l'ensemble des homéomorphismes $f$ de $\mathbb{S}^{1}$ tels que: il existe une suite finie $x_{1}<\cdots<x_{n}<x_{1}$ d'éléments de $\widehat{\mathbb{Q}}$ tels que $f$ est la restriction d'un élément de $P S L_{2} \mathbb{Z}$, noté $f_{i}$ sur chaque intervalle $\left[x_{i} ; x_{i+1}\right]$.

Proposition I.8. PPSL $L_{2} \mathbb{Z}$ est un sous-groupe du groupe des difféomorphismes du cercle de classe $\mathcal{C}^{1}$. 
Preuve. - L'invariance de $P P S L_{2} \mathbb{Z}$ par composition et par passage à l'inverse provient du fait que l'ensemble des rationnels $\widehat{\mathbb{Q}}$ est stable par $P S L_{2} \mathbb{Z}$. Pour montrer qu'un élément $f$ de $P P S L_{2} \mathbb{Z}$ est de classe $\mathcal{C}^{1}$ en $x \in \mathbb{S}^{1}$, on peut supposer, quitte à conjuguer $f$ par un élément de $P S L_{2} \mathbb{Z}$, que $x=0$, puis que $f$ fixe 0 .

Notons pour $i \in\{l ; r\} f_{0}^{i}(z)=\frac{a_{i} z+b_{i}}{c_{i} z+d_{i}}$ avec la condition $a_{i} d_{i}-b_{i} c_{i}=1$. On a alors $0=f_{0}^{i}(0)=\frac{b_{i}}{d_{i}}$ ce qui entraine l'égalité $d_{r}= \pm d_{l}= \pm 1$ puisque $a_{i} d_{i}=1$. Puis, on a $\left(f_{0}^{i}\right)^{\prime}(0)=\frac{1}{d_{i}{ }^{2}}=1$ et $f$ est bien de classe $\mathcal{C}^{1}$ au voisinage de 0 .

Considérons à présent la bijection $i$ entre $\widehat{\mathbb{Q}}$ et $\mathbb{Q}(2)$ donnée en section I.1.c. Par densité de $\widehat{\mathbb{Q}}$ dans $\mathbb{R} \cup\{\infty\}$ et de $\mathbb{Q}(2)$ dans $\mathbb{R} / \mathbb{Z}$, l'application $i$ est prolongé en un homéomorphisme de $\mathbb{R} \cup\{\infty\}$ vers $\mathbb{R} / \mathbb{Z}$, noté $i$ aussi. On peut trouver une démonstration du résultat suivant dans [Imb].

Proposition I.9. Le conjugué de PPSL $L_{2} \mathbb{Z}$ par $i$ est exactement le groupe de Thompson $P L_{2}\left(\mathbb{S}^{1}\right)$.

\section{Le groupe de Ptolémée}

Rappelons qu'une tesselation marquée $\delta$ ou plus simplement tesselation est une triangulation maximale du disque de Poincaré équipée d'une arête orientée distinguée. Une tesselation rationnelle est une tesselation dont l'ensemble des sommets est exactement $\widehat{\mathbb{Q}}=\tau_{*}^{(0)}$.

Nous reprenons l'article de R. Penner [Pen3] pour définir le groupe de Ptolémée en trois étapes: groupoïde, monoïde, et enfin groupe.

Soit $\tau$ une tesselation et $\gamma$ une de ses arêtes. On définit une tesselation $\tau_{\gamma}$ selon deux cas:

(1) si $\gamma$ est l'arête distinguée, on la remplace par la seconde diagonale $\gamma *$ du quadrilatère défini par $\tau$ et contenant $\gamma$, et on oriente $\gamma *$ de sorte que $(\gamma ; \gamma *)$ forme un angle orienté positivement.

(2) si $\gamma$ est différente de l'arête distinguée, on la remplace également par $\gamma *$ et on prend pour arête orientée de $\tau_{\gamma}$ celle de $\tau$.

Les autres arêtes sont preservées.

Cette opération s'appelle mouvement élémentaire le long de $\gamma \in \tau$.

Définition I.6.3. (Groupoïde de Ptolémée)

Soit $P t^{\prime}$ la catégorie suivante:

- Objets: les tesselations marquées

- Morphismes: un morphisme de $\tau$ à $\delta$ est une suite finie $\tau=\tau_{1}, \cdots, \tau_{n}=\delta$ telle que $\tau_{i+1}$ est obtenu par mouvement élémentaire le long de $\gamma_{i} \in \tau_{i}$. En passant aux objets modulo $P S L_{2} \mathbb{R}$, on obtient le groupoïde de Ptolémée $P t$.

Il y a une façon canonique de passer d'un groupoïde à un groupe, via une structure de monoïde, lorsque les objets de cette catégorie sont isomorphes. Rappelons qu'ils le sont par les applications caractéristiques $f_{\tau}: \tau_{*} \rightarrow \tau$, définies à partir de leur arête 
distinguée. Prenons $\tau_{*}$ pour objet base. On note $M$ le monoïde libre sur $\tau_{*}$ (ce sont les mots $q_{n} \cdots q_{1}$ où les $q_{i}$ sont des éléments de $\tau_{*}$ ). Si $q \in \tau_{*}$, notons $q \cdot \tau$ la tesselation obtenue après mouvement élémentaire de $\tau$ le long de $f_{\tau}(q) \in \tau$.

On a ainsi une action à gauche de $M$ sur Tess' définie par

$$
\left(q_{n} \cdots q_{1}\right) \cdot \tau=q_{n} \cdot\left(\cdots q_{2} \cdot\left(q_{1} \cdot \tau\right) \cdots\right)
$$

qui passe au quotient en une action sur l'espace Tess.

Soit $K$ le sous-monoïde de $M$ constitué des éléments qui agissent sur Tess' en préservant $\tau_{*}$. Le groupe de Ptolémée universel $G$ est défini comme le quotient de $M$ par $K$. Pour montrer que $G$ est un groupe, il suffit de voir que $K$ est suffisamment grand pour pouvoir trouver un inverse à tous les éléments de $M / K$. Par exemple, si $\alpha$ est le mouvement élémentaire le long de l'arête distinguée de $\tau_{*}, \alpha^{4} \in K$. Par conséquent le mot $\alpha^{3}$ est un inverse de $\alpha$ dans $G$.

Considérons alors $\gamma \in G$, et $\tau_{\gamma}=\gamma \cdot \tau_{*}$. D'après le théorème I.1, il existe un unique homéomorphisme $f_{\gamma}$ associé à $\tau_{\gamma}$. Cette construction fournit un morphisme de $G$ dans Homeo $^{+}\left(\mathbb{S}^{1}\right)$. D'après une remarque de M.Kontsevich, on a le:

Théorème I.2. (Imbert [Imb]) La représentation $G \rightarrow \mathrm{Homeo}^{+}\left(\mathbb{S}^{1}\right)$ qui à $\gamma$ associe $f_{\gamma}$ réalise un isomorphisme sur le groupe $T$.

Nous utiliserons cette représentation du groupe de Thompson $T$ dans les chapitres III et IV.

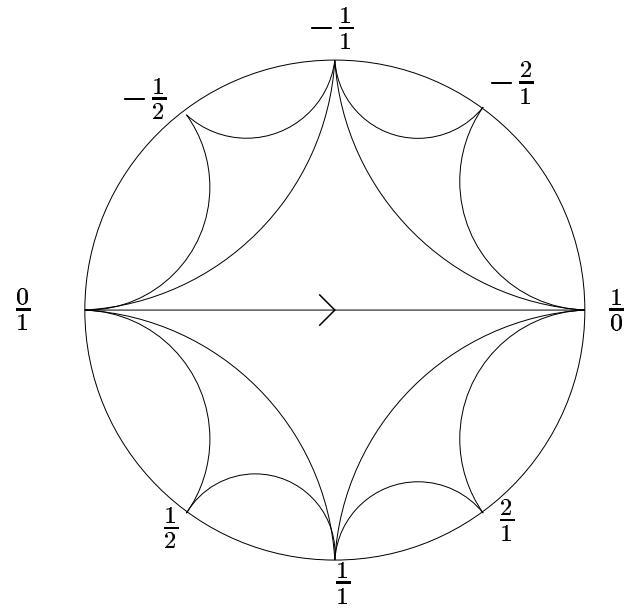

FIG. I.8 - Tesselation de Farey. 


\section{Chapitre II}

\section{Les systèmes de coordonnées sur $\mathcal{G} r$ et $\mathcal{B} r$}

L'espace de Teichmüller $\mathcal{T}_{g}$ d'une surface de genre $g \geq 2$ possède de nombreux systèmes de coordonnées [Abi]. Par exemple, les coordonnées de Fenchel-Nielsen fournissent un homéomorphisme entre $\mathcal{T}_{g}$ et $\mathbb{R}^{6 g-6}$. Ce qui prouve au passage que l'espace $\mathcal{T}_{g}$ est contractile. Ces coordonnées permettent aussi de comprendre l'action du groupe $M C_{g}$ des difféotopies de la surface sur l'espace de Teichmuller, appelé aussi "Mapping Class Group". Le quotient est l'espace des modules de la surface (espace des structures conformes à difféomorphismes conformes près).

Dans le cadre universel, l'espace de Teichmüller universel de Bers:

$$
T(1)=P S L_{2} \mathbb{R} \backslash\left\{\text { homéomorphismes quasi-symétriques de } \mathbb{S}^{1}\right\}=P S L_{2} \mathbb{R} \backslash Q S
$$

est plongé sur un domaine convexe holomorphe dans un espace de Banach complexe. En particulier $T(1)$ est contractile [Ear-Eel].

Pour l'étude de l'espace $\mathcal{G} r$, on le plonge dans un espace de paramètres, afin de montrer qu'il est contractile. Plus précisément dans ce chapitre, nous décrivons deux systèmes de coordonnées sur $\mathcal{G} r$ :

- Les coordonnées log-lambda.

- Les coordonnées horocycliques.

Dans la section II.1, on s'intéresse au premier système. Celui-ci est défini sur l'espace des tesselations décorées $\widetilde{\text { Tess }}\left[\right.$ Pen2]. Il se restreint à l'espace $P S L_{2} \mathbb{R} \backslash C P P$ via le plongement de la proposition I.6. Dans ce système de coordonnées, l'espace $\mathcal{G} r$ prend une formulation très simple. C'est une sorte de limite directe $\lim _{n \rightarrow \infty} \mathbb{R}^{3\left(2^{n}-1\right)}$ (théorème principal II.2). La contractibilité de l'espace $\mathcal{G} r$ (dont une esquisse de preuve est donnée dans [Gre4]) en est une conséquence immédiate. Les techniques utilisant les déformations log-lambda seront adaptées à l'étude de l'espace des bracelets propres $\mathcal{B}_{n}$. On donnera une nouvelle démonstration de la contractibilité de $\mathcal{B}_{n}$ (théorème II.4).

Dans la section II.2, on étudie le système des coordonnées horocycliques, à l'origine décrit dans l'appendice "Géographie des bracelets" de [Gre3]. Nous donnons une démonstration du "Résultat" A.1 de cet appendice (proposition II.3). 
Un des intérets de ce système, est la définition d'une métrique $d^{b}$, dans la section II.3. Celle-ci fournit une autre topologie à l'espace $\mathcal{G} r$. Nous verrons dans le chapitre III que $d^{b}$ est invariante par le groupe de Thompson $T$.

\section{II.1 Coordonnées log-lambda}

Les coordonnées log-lambda ont été introduites par Penner [Pen1] en 1987. Elle fournissent, une paramêtrisation de l'espace de Teichmûller décoré d'une surface piquée. Dans [Pen2], le même auteur étend ces coordonnées au cas universel $\widetilde{\text { Tess. Elles }}$ fournissent un système de coordonnées affines global de l'espace de Teichmüller universel décoré $\widetilde{\text { Tess }}$ (théorème II.1). Dans ce système de coordonnées, on peut montrer que l'espace $\widetilde{\text { Tess }}$ (introduit en I.4) est connexe par arcs [Pen2]. Ce système de coordonnées amène des questions intéressantes. La suivante semble la plus naturelle: Quel est le lieu des points de $\widetilde{\text { Tess }}$ dans ce système de coordonnées?

En restriction à l'espace $\mathcal{G} r$, via le plongement $s$ du corollaire I.2, on donne le lieu exact de $\mathcal{G} r$ dans les coordonnées log-lambda (théorème II.2). On en déduit alors la contractibilité de $\mathcal{G} r$.

Enfin toujours à l'aide des coordonnées log-lambda, on redémontre la contractibilité de l'espace des $(n+1)$-bracelets propres modulo $P S L_{2} \mathbb{R}, \mathcal{B}_{n}$ (théorème II.4).

\section{II.1.a Les coordonnées sur l'espace $\widetilde{\text { Tess }}$}

Soit $e=(x ; y)$ une géodésique de $\mathbb{D}^{2}$ décorée, c'est à dire portant un couple d'horocycles $h=\left(h_{x}, h_{y}\right)$ (la décoration) en ses extrémités.

Plus généralement, soit $I^{(0)}$ un sous-ensemble du cercle décoré par une collection d'horocycles $h=\left(h_{x}\right)_{x \in I^{(0)}}$ et notons $I^{(1)}$ l'ensemble des géodésiques de $\mathbb{D}^{2}$ dont les extrémités sont dans $I^{(0)}$. Lorsque $\tilde{\tau}$ est une tesselation décorée, avec $I^{(0)}=\tau_{*}^{(0)}$, alors l'ensemble des arêtes de $\tau$ est contenu dans l'ensemble $I^{(1)}$.

Définition II.1.1. (Lambda-longueur)

La quantité:

$$
\Lambda(h ; e)=\sqrt{2 \cdot \exp \left(-\frac{d_{h}\left(h_{x}, h_{y}\right)}{2}\right)}
$$

s'appelle la lambda-longueur de $h$ le long de $e$.

De même, si $I^{(0)} \subset \mathbb{S}^{1}$ est décoré par $h=\left(h_{x}\right)_{x \in I^{(0)}}$, et $e=(x ; y)$ un élément de $I^{(1)}$, la quantité $\Lambda(h ; e)$ s'appelle la lambda-longueur de $h$ le long de $e$.

Dans le cas particulier d'une tesselation décorée $\tilde{\tau}$, contenant une arête $e$, cette quantité sera notée $\Lambda(\tilde{\tau} ; e)$ et appelée lambda-longueur de $\tilde{\tau}$ le long de $e$.

Les lambda-longueurs permettent de définir l'application: 


$$
\begin{aligned}
& E: \widetilde{\text { Tess }}: \longrightarrow \mathbb{R}_{+}^{\tau_{*}} \\
& \tilde{\tau} \quad \longmapsto E_{\tilde{\tau}}: \tau_{*} \longrightarrow \mathbb{R}_{+} \\
& e \longmapsto \Lambda\left(\tilde{\tau}, f_{\tau}(e)\right)
\end{aligned}
$$

où $f_{\tau}$ est l'application caractéristique de $\tau_{*}$ vers $\tau$ construite au paragraphe I.4.b. On a le théorème suivant:

Théorème II.1 (Penner, [Pen2]). L'application E réalise un plongement de $\widetilde{\text { Tess }}$ dans $\mathbb{R}_{+}^{\tau_{*}}$, muni de la topologie produit.

Cela munit ainsi $\widetilde{\text { Tess }}$ d'un système de coordonnées affines global, les coordonnées log-lambda (ou coordonnées de Penner), portées par les arêtes de la tesselation de Farey.

Dans les coordonnées de Penner, la tesselation décorée $\tilde{\tau}_{*}$ correspond à l'application constante égale à $\sqrt{2}$. Dans le paragraphe I.5.a, nous avons plongé $\mathcal{G} r$ dans l'espace $\widetilde{T e s s}$. Nous sommes en mesure à présent d'identifier l'espace $\mathcal{G} r$ dans le système des coordonnées de Penner. Cela nous permettra ensuite de montrer sa contractibilité.

Avant de continuer, nous citons un lemme important qui donne l'injectivité de l'application $E$ dans la démonstration du théorème II.1. Nous donnons une preuve géométrique de ce lemme, démontré par Penner de façon analytique.

Lemme II.1. Soit $h_{1}, h_{3}$ deux horocycles de $\mathbb{D}^{2}$ et $\lambda_{3}, \lambda_{1}$ deux réels strictement positifs. Alors, il existe un unique horocycle $h_{2}$ (basé en $x_{2}$ ) à droite de l'arête orientée $\left(x_{1} ; x_{3}\right)$ tel que les lambda-longueurs des arêtes du triangle $\left(x_{1} ; x_{2} ; x_{3}\right)$ pour la décoration $\left(h_{1}, h_{2}, h_{3}\right)$ vérifient:

$$
\Lambda_{3}=\lambda_{3}, \Lambda_{1}=\lambda_{1}
$$

où $\Lambda_{i}$ est la lambda-longueur de l'arête opposée à l'horocycle $h_{i}$.

Preuve. - La démonstration est similaire à celle de la proposition I.5. Elle est contenue dans la figure II.1:
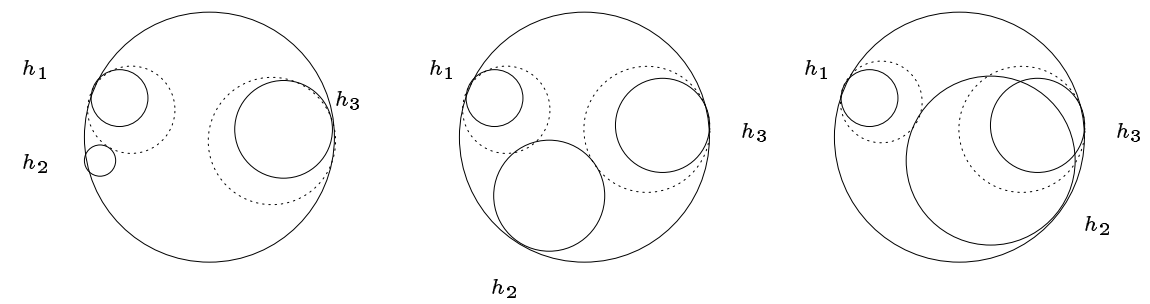

Fig. II.1 - Unicité de $h_{2}$.

L'horocycle $h_{1}^{\prime}$ (resp. $h_{3}^{\prime}$ ) déssiné avec une ligne discontinue et basé en $x_{1}$ (resp. en $x_{3}$ ) représente le lieu des points à distance $\lambda_{3}$ de $h_{1}$ (resp. $\lambda_{1}$ de $h_{3}$ ). Notons $\left(h_{x}\right)_{x \in] x_{1} ; x_{3}[}$ la famille d'horocycles définie de façon unique par la condition que $h_{x}$ est tangent à $h_{1}^{\prime}$. Lorsque $x$ se déplace de $x_{1}$ à $x_{3}$, le rayon euclidien de $h_{x}$ augmente de sorte qu'il existe un unique $x=x_{2}$ tel que $h_{x}$ soit tangent à $h_{3}^{\prime}$. L'horocycle $h_{x_{2}}$ est ainsi l'unique horocycle vérifiant les conditions du lemme. 


\section{II.1.b Une coordonnée $=$ Une déformation log-lambda}

Soit $\tau$ une tesselation du disque de Poincaré et $e=\left(x_{1} ; x_{3}\right)$ une arête de $\tau$. L'arête $e$ est bord de deux triangles de la tesselation dont l'union forme un quadrilatère $Q_{\tau, e}$ de $\mathbb{D}^{2}$. L'ensemble des sommets du quadrilatère forme la configuration ordonnée $\left(x_{1}, x_{2}, x_{3}, x_{4}\right)$. On note alors $\widetilde{\theta}_{t, \tau, e}$ la déformation log-lambda, définie au paragraphe I.2.b, relativement au quadrilatère $Q_{\tau, e}$ et de rapport $t$. La figure suivante représente une telle déformation dans le plan hyperbolique.

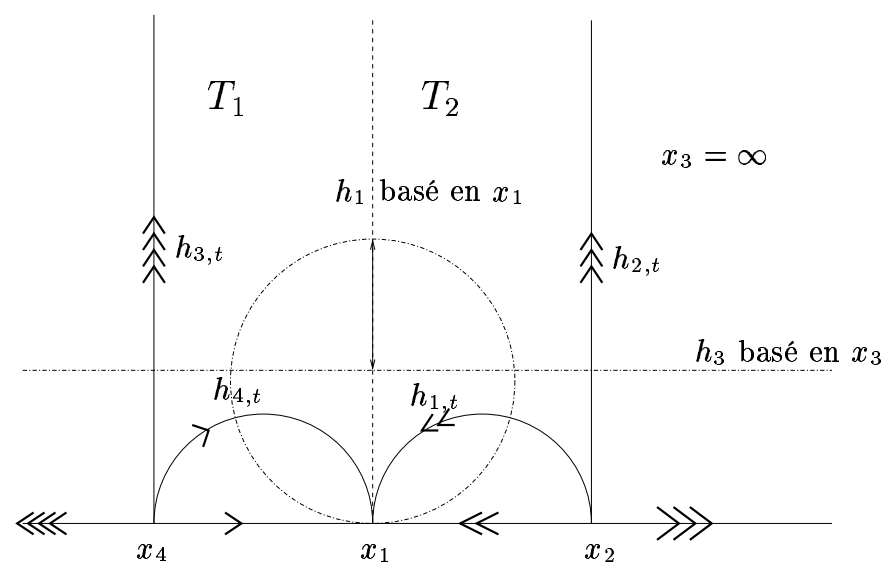

FIG. II.2 - Déformation log-lambda.

La proposition suivante précise la nature des déformations log-lambda. En particulier, le point 2 . fournit un lien entre coordonnées log-lambda et déformations loglambda.

Proposition II.1. Les déformations log-lambda vérifient les propriétés suivantes:

1. $\left(\widetilde{\theta}_{t, \tau, e}\right)_{t \in \mathbb{R}_{+}^{*}}$ est un sous-groupe à un paramètre de CPP isomorphe à $\mathbb{R}_{+}^{*}$. Si $\tau=\tau_{*}$, alors ce sous-groupe de CPP est en fait dans $\widehat{\mathcal{G} r}$.

2. Si e est une arête de $\tilde{\tau}$, en terme de lambda-longueurs en posant $\Lambda_{t}\left(e^{\prime}\right)=\Lambda\left(\widetilde{\theta}_{t, \tau_{*}, e}\right.$. $\left.\tilde{\tau} ; \widetilde{\theta}_{t, \tau_{*}, e}\left(e^{\prime}\right)\right)$, on a les égalités:

$$
\begin{gathered}
\text { 1. } \Lambda_{t}(e)=t \Lambda(\tilde{\tau}, e) \\
\text { 2. } \Lambda_{t}\left(e^{\prime}\right)=\Lambda\left(\tilde{\tau}, e^{\prime}\right) \quad \forall e^{\prime} \in \tau \backslash\{e\}
\end{gathered}
$$

Preuve. -

1. Ceci résulte de la définition des déformations log-lambda.

2. Quitte à conjuguer la déformation par un élément de $P S L_{2}(\mathbb{R})$ envoyant le couple $\left(x_{1} ; x_{3}\right)$ sur $(0 ; \infty)$, on peut supposer $x_{1}=0$ et $x_{3}=\infty$.

L'application $\widetilde{\theta}_{t, \tau, e}$ agit comme l'application $z \mapsto t^{2} z$ sur l'ensemble des horocycles $h_{1}$ basés en $x_{1}$ et comme $z \mapsto \frac{z}{t^{2}}$ sur l'ensemble des horocycles $h_{3}$ basés en $x_{3}$. Si 
$h_{i}^{t}=\widetilde{\theta}_{t, \tau, e}\left(h_{i}\right)$ pour $i \in<1 ; 4>$ on a:

$$
d\left(h_{1}^{t}, h_{3}^{t}\right)=\int_{\frac{a_{3}}{t^{2}}}^{t^{2} a_{1}} \frac{d y}{y}=2 \log t^{2}+\left(\log a_{1}-\log a_{3}\right)
$$

On en déduit que:

$$
\begin{gathered}
\Lambda_{t}(e)=\sqrt{2 \exp \left(-\frac{d\left(h_{1}^{t}, h_{3}^{t}\right)}{2}\right)}=t \sqrt{2 \exp \left(-\frac{\log a_{1}-\log a_{3}}{2}\right)}= \\
=t \sqrt{2 \exp \left(-\frac{d\left(h_{1}, h_{3}\right)}{2}\right)}=t \Lambda(\tilde{\tau}, e)
\end{gathered}
$$

Par ailleurs, si $e^{\prime} \in \tau \backslash\{e\}$ ses extrémités sont dans un intervalle sur lequel $\widetilde{\theta}_{t, \tau, e}$ agit comme $P S L_{2}(\mathbb{R})$, donc $\Lambda_{t}\left(e^{\prime}\right)=\Lambda(\tilde{\tau}, e)$.

\section{II.1.c Le théorème principal: lambda-coordonnées sur $\mathcal{G} r$}

Soit $\left(S_{n}\right)_{n \in \mathbb{N} *}$ la filtration de $\widehat{\mathbb{Q}}$ introduite au paragraphe I.1.b. Elle définit une filtration $\tau_{*}=\cup_{n \geq 2}\left(\tau_{*}\right)_{n}$ en posant:

$$
\left(\tau_{*}\right)_{n}=\left\{e=(x ; y) \in \tau_{*} \mid(x, y) \in\left(S_{n-1}\right)^{2}\right\} \quad \forall n \geq 2
$$

Si $\Lambda$ est un élément de l'espace des paramètres $\mathbb{R}_{+}^{*} \tau_{*}$, on note:

$$
\operatorname{Supp}(\Lambda)=\left\{e \in \tau_{*}^{(0)} \mid \Lambda(e) \neq \sqrt{2}\right\} \subset \tau_{*}^{(0)}
$$

le support de $\Lambda$. On définit alors les sous-espaces de $\mathbb{R}_{+}^{*} \tau_{*}$ suivants:

$$
\begin{gathered}
\left(\mathbb{R}_{+}^{*}\right)_{\sqrt{2}}^{\tau_{*}}=\left\{\Lambda \in \mathbb{R}_{+}^{*} \tau_{*} \mid \operatorname{card}(\operatorname{Supp}(\Lambda))<\infty\right\} \\
\left(\mathbb{R}_{+}^{*}\right)_{\sqrt{2}}^{\left(\tau_{*}\right)_{n}}=\left\{\Lambda \in \mathbb{R}_{+}^{* \tau_{*}} \mid \operatorname{Supp}(\Lambda) \subset\left(\tau_{*}\right)_{n}\right\}
\end{gathered}
$$

L'espace $\left(\mathbb{R}_{+}^{*}\right)_{\sqrt{2}}^{\left(\tau_{*}\right)_{n}}$ est homéomorphe à l'espace euclidien réel de dimension $\operatorname{card}\left(\left(\tau_{*}\right)_{n}\right)=3\left(2^{n-1}-1\right)$. L'espace $\left(\mathbb{R}_{+}^{*}\right)_{\sqrt{2}}^{\tau_{*}}$ est ensemblistement la limite directe des espaces $\left(\mathbb{R}_{+}^{*}\right)_{\sqrt{2}}^{\left(\tau_{*}\right)_{n}}$, via les applications d'inclusion $\left(\mathbb{R}_{+}^{*}\right)_{\sqrt{2}}^{\left(\tau_{*}\right)_{n}} \subset\left(\mathbb{R}_{+}^{*}\right)_{\sqrt{2}}^{\left(\tau_{*}\right)_{n+1}}$. On le munit de la topologie faible.

Soit $\Lambda: \mathcal{G} r \rightarrow \mathbb{R}_{+}^{*} \tau_{*}$ l'application composée $E \circ s$ (définies respectivement en I.5.a et II.1.a) et $\Lambda_{n}$ sa restriction à $\mathcal{G} r_{n}$.

Théorème II.2. L'application $\Lambda$ réalise un homéomorphisme de $\mathcal{G} r$ sur $\left(\mathbb{R}_{+}^{*}\right)_{\sqrt{2}}^{\tau_{*}}$, munis de la topologie faible. 
Preuve. - On commence par démontrer l'égalite $\Lambda(\mathcal{G} r)=\left(\mathbb{R}_{+}^{*}\right)_{\sqrt{2}}^{\tau_{*}}$ grâce aux filtrations respectives de $\mathcal{G} r$ et $\left(\mathbb{R}_{+}^{*}\right)_{\sqrt{2}}^{\tau_{*}}$. Montrons le:

Lemme II.2. L'image de $\mathcal{G} r_{n}$ par $\Lambda_{n}$ est exactement $\left(\mathbb{R}_{+}^{*}\right)_{\sqrt{2}}^{\left(\tau_{*}\right)_{n}}$.

Preuve. - On procède par inclusions réciproques.

- $\subset$ : Si $e=(x ; y) \in \tau_{*} \backslash\left(\tau_{*}\right)_{n}$, alors ses extrémités sont contenues dans un intervalle $\left[x_{i} ; x_{i+1}\right]$ délimité par deux points consécutifs de $S_{n}$. De plus, la restriction d'un élément $f$ de $\mathcal{G} r_{n}$ sur cet intervalle coïncide avec un élément $h_{i}$ de $P S L_{2} \mathbb{R}$. Par conséquent, $d_{h}\left(f \cdot h_{x}^{F} ; f \cdot h_{y}^{F}\right)=d_{h}\left(h_{i}\left(h_{x} F\right) ; h_{i}\left(h_{y}^{F}\right)\right)=d_{h}\left(h_{x}^{F} ; h_{y}^{F}\right)$. Il s'en suit l'égalité $\Lambda(f)(e)=\sqrt{2}$ et celle-ci est vraie pour tout couple $(f ; e) \in \mathcal{G} r_{n} \times \tau_{*} \backslash\left(\tau_{*}\right)_{n}$. Formulé autrement, on a $\Lambda\left(\mathcal{G} r_{n}\right) \subset\left(\mathbb{R}_{+}^{*}\right)_{\sqrt{2}}^{\left(\tau_{*}\right)_{n}}$.

- $\supset$ : Pour l'inclusion réciproque, on définit un ordre naturel sur $\tau_{*}$ par récurrence sur $n$ en partant de l'arête distinguée et en se déplacant sur le cercle (en suivant l'orientation du cercle à partir 0 ).

- Le rang 1:

$$
e_{1}=\left(\frac{0}{1} ; \frac{1}{0}\right)<e_{2}=\left(\frac{1}{0} ;-\frac{1}{1}\right)<e_{3}=\left(-\frac{1}{1} ; \frac{0}{1}\right)
$$

- Du rang $n$ au rang $n+1$ :

Supposons l'ordre construit sur $\left(\tau_{*}\right)_{n}$. L'ensemble $\left(\tau_{*}\right)_{n+1} \backslash\left(\tau_{*}\right)_{n}$ forme un $3 \cdot 2^{n-1}$ gone hyperbolique, dont l'ensemble des sommets est la configuration ordonnée $S_{n}=$ $\left(v_{1}, \cdots, v_{3 \cdot 2^{n-1}}\right)$ définie par $v_{1}=0$. Les arêtes de ce polygone sont les géodésiques $a_{i}=\left(v_{i} ; v_{i+1}\right)$ pour $i \in<1 ; 3 \cdot 2^{n-1}>\left(\right.$ avec la convention $\left.v_{3 \cdot 2^{n-1}+1}=v_{1}\right)$. Ainsi $\left(\tau_{*}\right)_{n+1} \backslash\left(\tau_{*}\right)_{n}$ est muni de l'ordre suivant: $a_{i}<a_{j}$ si et seulement si $i<j$. Pour l'ordre sur $\left(\tau_{*}\right)_{n+1}$, on stipule que le plus grand élément de $\left(\tau_{*}\right)_{n}$ (l'hypothèse de récurrence suppose un ordre sur $\left(\tau_{*}\right)_{n}$ ) est plus petit que $a_{1}$, ce qui fournit un ordre naturel à l'ensemble $\left(\tau_{*}\right)_{n+1}$.

Passons à la démonstration de l'inclusion réciproque. Soit $\left(t_{1} \sqrt{2}, \cdots, t_{3\left(2^{n-1}-1\right)} \sqrt{2}, \sqrt{2}, \cdots\right)$ un élément de $\left(\mathbb{R}_{+}^{*}\right)_{\sqrt{2}}^{\left(\tau_{*}\right)_{n}}$. Muni de cet ordre naturel, on construit par récurrence sur l'ordre (noté $k$ ) de $\left(\tau_{*}\right)_{n}$ une suite d'applications $\left(f_{i}\right)_{i \in<1 ; k>} \in C P P^{k}$ tels que: $g_{k}=f_{k} \circ \cdots f_{1} \in \mathcal{G} r_{n}$ et $\Lambda\left(g_{k}\right)=\left(t_{1} \sqrt{2}, \cdots, t_{k} \sqrt{2}, \sqrt{2}, \cdots\right)$. - Le rang 1: Soit $g_{1}$ le normalisé de la déformation log-lambda $\widetilde{\theta}_{t_{1}, \tau_{*}, e_{1}}$. D'après l'assertion 3 de la proposition II.1, on obtient $\Lambda\left(g_{1}\right)\left(e_{1}\right)=t_{1} \sqrt{2}$ et $\Lambda\left(g_{1}\right)(e)=\sqrt{2}$ si $e \neq e_{1}$. - Le passage du rang $k$ au rang $k+1$ : Supposons donc avoir construit une suite d'applications $\left(f_{i}\right)_{i \in<1 ; k>}$ vérifiant l'hypothèse de récurrence, on définit $f_{k+1}$ comme le nor-

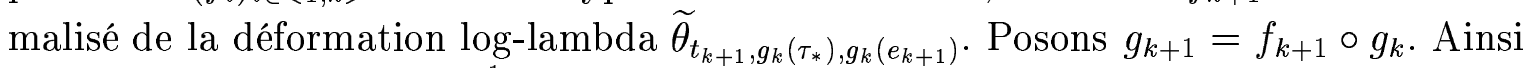
on a $b k\left(g_{k+1}\right) \subset b k\left(g_{k}\right) \cup g_{k}^{-1}\left(b k\left(f_{k+1}\right)\right)$. Par hypothèse de récurrence, on a $b k\left(g_{k}\right) \subset S_{n}$ et puisque le quadrilatère associé à $e$ a ses extrémités dans $S_{n}$, on a $b k\left(f_{k+1}\right) \subset g_{k}\left(S_{n}\right)$. On en déduit que $g_{k+1}$ est dans $\mathcal{G} r_{n}$. Il reste à calculer les coordonnées log-lambda de $g_{k+1}$.

Par définition, on a:

$$
\Lambda\left(g_{k+1}\right)(e)=\Lambda\left(f_{k+1} \circ g_{k}\right)(e)=\Lambda\left(\left(f_{k+1} \circ g_{k}\right)\left(\tilde{\tau}_{*}\right) ;\left(f_{k+1} \circ g_{k}\right)(e)\right)
$$


D'après l'assertion 3. de la proposition II.1, le dernier terme est égal à:

$$
\Lambda\left(g_{k} \cdot \tilde{\tau}_{*} ; g_{k}(e)\right) \text { si } g_{k}(e) \neq g_{k}\left(e_{k+1}\right)
$$

et

$$
t_{k+1} \Lambda\left(g_{k} \cdot \tilde{\tau}_{*} ; g_{k}(e)\right) \text { si } g_{k}(e)=g_{k}\left(e_{k+1}\right)
$$

On obtient ainsi, par hypothèse de récurrence sur les coordonnées de $g_{k}$,

$$
\Lambda\left(g_{k+1}\right)=\left(t_{1} \sqrt{2}, \cdots, t_{k} \sqrt{2}, t_{k+1} \sqrt{2}, \sqrt{2}, \cdots\right)
$$

ce qui montre que l'assertion est vérifiée au rang $k+1$ et conclut la démonstration du lemme.

Revenons à la preuve du théorème. Puisque $\left(\mathbb{R}_{+}^{*}\right)_{\sqrt{2}}^{\tau_{*}}$ est la limite directe des espaces $\left(\mathbb{R}_{+}^{*}\right)_{\sqrt{2}}^{\left(\tau_{*}\right)_{n}}$, on en déduit la surjectivité de l'application $\Lambda$. De plus, on sait déjà que $\Lambda$ est injective. C'est donc une bijection de $\mathcal{G} r$ vers $\left(\mathbb{R}_{+}^{*}\right)_{\sqrt{2}}^{\tau_{*}}$.

Montrons que $\Lambda_{n}$ fournit un homéomorphisme entre $\mathcal{G} r_{n}$ et $\left(\mathbb{R}_{+}^{*}\right)_{\sqrt{2}}^{\left(\tau_{*}\right)_{n}}$. Puisque chaque composante $\Lambda_{n}(e): f \in \mathcal{G} r_{n} \mapsto \Lambda(f)(e) \in \mathbb{R}_{+}^{*}$ est continue pour $e \in\left(\tau_{*}\right)_{n}$, l'application $\Lambda_{n}$ est également continue. Supposons maintenant que $\left(f_{p}\right)_{p \in \mathbb{N}}$ et $f$ sont des éléments de $\mathcal{G} r_{n}$ tels que $\Lambda_{n}\left(f_{p}\right)$ converge vers $\Lambda_{n}(f)$. Comme $\Lambda\left(f_{p}\right)(e)=\sqrt{2}=\Lambda(f)(e)$ pour tout $e \notin\left(\tau_{*}\right)_{n}, \Lambda\left(f_{p}\right)$ converge vers $\Lambda(f)$ pour la topologie produit de $\mathbb{R}_{+}^{*} \tau_{*}$. Par ailleurs, l'application $E: \widetilde{\text { Tess }} \rightarrow \mathbb{R}_{+}^{*} \tau_{*}$ est ouverte (théorème II.1.a). Ce qui implique que $s\left(f_{p}\right)$ converge vers $s(f)$. De plus, l'application $\widetilde{T e s s} \rightarrow$ Tess, qui consiste en l'oubli de la décoration est continue. On déduit alors du théorème I.1 que la suite de fonction $\left(f_{p}\right)$ converge uniformément vers $f$ sur le cercle. L'argument utilisant la proposition 4.5.4. dans [Bea], dans la démonstration du corollaire I.3 entraine la convergence en classe $\mathcal{C}^{1}$, ce qui montre que $\Lambda_{n}^{-1}$ est continue.

La proposition I.3 permet de conclure que $\Lambda$ est un homéomorphisme.

Corollaire II.1. L'espace $\mathcal{G} r$ muni de la topologie faible est contractile.

Preuve. - Cela découle directement de la proposition I.1 et du théorème II.2.

On retrouve ainsi le résultat énoncé dans [Gre4] pour lequel P. Greenberg donne seulement une esquisse de preuve. J'aimerais préciser ici que ce théorème est en fait le point de départ de cette thèse.

\section{II.1.d Les coordonnées sur les espaces de bracelets}

Ici, nous utilisons les coordonnées log-lambda dans le cadre de la géométrie des bracelets.

Dans [Gre3], l'auteur relie certains espaces de bracelets aux polytopes de Stasheff (ou associahédrons). Les associahedrons $\left(K_{n}\right)_{n \in \mathbb{N}}$, ont été introduit en 1963 dans [Sta]. Ces polytopes ont été construit afin de caractériser, les espaces dont le type d'homotopie sont ceux d'espaces de lacets. A l'origine, le polytope $K_{n}$ est décrit en termes 
de parenthésages possibles de $n$ lettres cycliquement ordonnées. De ce point vue, ce polytope est fortement relié au manque d'associativité de la composition des lacets. Notons enfin que le complexe de Stasheff est contractile.

Rappelons que $\widehat{\mathcal{B}_{n}}$ est l'espace des $(n+1)$-bracelets propres et $\mathcal{B}_{n}$ le sous-espace de $\widehat{\mathcal{B}_{n}}$ des bracelets $b=\left(h_{1} ; \cdots ; h_{n+1}\right)$ tels que $h_{1}$ (resp. $\left.h_{n+1}\right)$ est l'horocycle de Ford basé en 0 (resp. $\infty$ ). L'espace $\mathcal{B}_{n}$ est homéomorphe au quotient de $\widehat{\mathcal{B}_{n}}$ par $P S L_{2} \mathbb{R}$.

On a le théorème suivant:

Théorème II.3 (Greenberg [Gre3]). Il existe un homéomorphisme entre $\mathcal{B}_{n}$ et $K_{n}$.

En particulier, l'espace $\mathcal{B}_{n}$ est contractile.

\section{Contractibilité de l'espace $\mathcal{B}_{n}$}

Il est naturel de se demander s'il est possible de retrouver la contractibilité de l'espace $\mathcal{B}_{n}$ à l'aide d'objets tels que les déformations log-lambda, puisque celles-ci semblent naturellement adaptées aux espaces de bracelets. C'est le but de ce paragraphe. On exhibe ici une paramétrisation de l'espace $\mathcal{B}_{n}$ en termes de coordonnées log-lambda.

Soit $A_{n}=\left[\sqrt{2}, \infty\left[{ }^{n-2} \subset \mathbb{R}^{n-2}\right.\right.$. Si $b=\left(h_{1} ; \cdots ; h_{n+1}\right) \in \mathcal{B}_{n}$, notons $\lambda_{i}(b)$ la lambdalongueur de la collection $b$ le long de l'arête $\left(x_{1} ; x_{i}\right)$ pour $i \in<3 ; n>$. On a le:

\section{Théorème II.4. L'application}

$$
\begin{array}{rlc}
\Phi_{n}: \mathcal{B}_{n} & \rightarrow & \mathbb{R}^{n-2} \\
b & \mapsto & \left(\lambda_{i}(b)\right)_{i \in<3, n>}
\end{array}
$$

fournit un homéomorphisme sur un sous-espace contractile de $A_{n}$.

Preuve. - L'application $\Phi_{n}$ est à valeurs dans $A_{n}$ puisque les éléments de $\mathcal{B}_{n}$ sont des bracelets propres. Montrons que l'application est injective. Soit $\lambda=\left(\lambda_{3}, \cdots, \lambda_{n}\right)=$ $\Phi_{n}\left(b^{\prime}\right)=\Phi_{n}(b)$ où $b=\left(h_{1}, \cdots, h_{n+1}\right)$ (resp. $\left.b^{\prime}=\left(h_{1}^{\prime}, \cdots, h_{n+1}^{\prime}\right)\right)$ est un élément de $\mathcal{B}_{n}$. La normalisation de $\mathcal{B}_{n}$ entraine que $h_{1}=h_{1}^{\prime}$ et $h_{n+1}=h_{n+1}^{\prime}$. Puis, le résultat d'unicité du lemme II.1, appliqué aux triplets $\left(h_{1}, h_{n}, h_{n+1}\right)$ et $\left(h_{1}, h_{n}^{\prime}, h_{n+1}\right)$, entraine l'égalité $h_{n}=h_{n}^{\prime}$. En appliquant toujours le lemme II.1, on montre par récurrence que $b=b^{\prime}$ et l'application $\Phi_{n}$ est injective.

La continuité et la continuité inverse de $\Phi_{n}$ (restreinte au but) proviennent du corollaire I.3 et du théorème II.2. Ainsi, $\Phi_{n}$ est un homéomorphisme sur son image.

Démontrons que $\mathcal{B}_{n}$ est contractile par récurrence sur $n . \mathcal{B}_{2}$ est contractile car il est réduit à un point. Supposons que l'espace $\mathcal{B}_{n}$ est contractile et définissons le sous-espace de $\mathcal{B}_{n+1}$ suivant:

$$
\mathcal{B}_{n}^{\prime}=\left\{b \in \mathcal{B}_{n+1} \mid \lambda_{n+1}(b)=\sqrt{2}\right\}
$$

Celui-ci est homéomorphe à l'espace $\mathcal{B}_{n}$. Il suffit donc de retracter par déformation $\mathcal{B}_{n+1} \operatorname{sur} \mathcal{B}_{n}^{\prime}$. 
Soit $b \in \mathcal{B}_{n+1}$ et $\theta_{t}^{b}$ la déformation log-lambda le long de l'arête $\left(x_{1} ; x_{n+1}\right)$ relativement au quadrilatère dont les sommets sont les points-base des horocycles $h_{1}, h_{n}, h_{n+1}$ et $h_{n+2}$ associés à $b$. Si $h_{t}^{b}$ est la transformation hyperbolique coïncidant avec $\theta_{t}^{b}$ sur l'intervalle $[\infty ; 0]$ (attention à l'orientation), on définit l'application suivante:

$$
\begin{array}{ccc}
\phi:[0 ; 1] \times \mathcal{B}_{n+1} & \rightarrow & \mathcal{B}_{n+1} \\
(t, b) & \mapsto \quad\left(\left(h_{a(t, b)}^{b}\right)^{-1} \circ \theta_{a(t, b)}^{b}\right) \cdot b
\end{array}
$$

où $a(t, b)=\left[\frac{\sqrt{2}}{\lambda_{n+1}(b)}\right]^{t}$ est une fonction continue sur $[0 ; 1] \times \mathcal{B}_{n+1}$ puisque $\lambda_{n+1}(b)>0$ sur $\mathcal{B}_{n+1}$. La figure II.3 montre une telle déformation. La déformation modifié $\left(h_{a(t, b)}^{b}\right)^{-1} \circ$ $\theta_{a(t, b)}^{b}$ est restreinte à l'identité sur l'intervalle [ $\left.\infty ; 0\right]$. Par conséquent, ce chemin est composé de bracelets normalisés, il est bien à valeurs dans $\mathcal{B}_{n+1}$.

L'application est continue et vérifie l'égalité $\lambda_{n+1}(\phi(1 ; b))=\sqrt{2}$ par définition de la fonction $a$ et d'après la proposition II.1. Ainsi $\phi(1, b) \in \mathcal{B}_{n}^{\prime}$ pour tout $b \in \mathcal{B}_{n+1}$. De plus, $\phi(0, b)=b$. On a prouvé que $\phi$ fournit une rétraction par déformation de $\mathcal{B}_{n+1}$ sur $\mathcal{B}_{n}^{\prime}$, ce qui termine la preuve.

Corollaire II.2. L'espace $\mathcal{B} r$ est contractile pour la topologie faible.

Preuve. - Le théorème II.4 implique en particulier que les espaces de bracelets $\mathcal{B} r_{n}$ sont contractiles. Un analogue de la proposition I.1 permet de conclure.

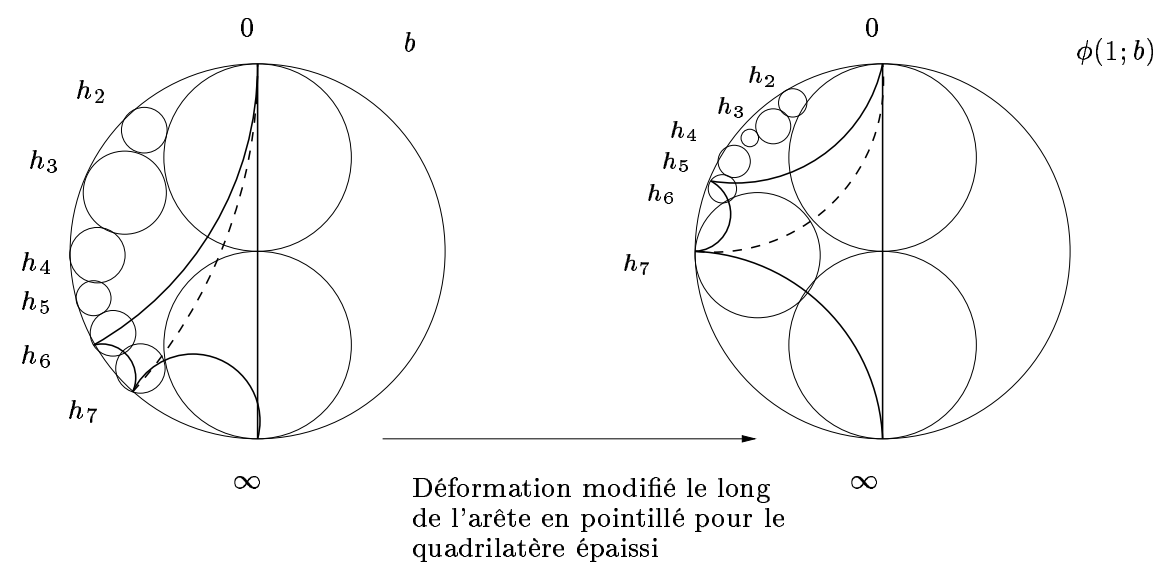

FIG. II.3 - Déformation log-lambda modifié.

\section{II.2 Coordonnées horocycliques}

Dans cette section, on fournit un autre système de coordonnées sur $\mathcal{G} r$, qui se définit dans le modèle des bracelets. Ces coordonnées ont été introduites dans [Gre3]. 
Elles sont spécifiques à la géométrie des bracelets. Nous montrons qu'elles sont liées au coordonnées log-lambda. Avant de les définir, nous rappelons un autre système de coordonnées de $\widehat{\text { Tess, }}$ qui fournit le lien entre coordonnées log-lambda et coordonnées horocycliques.

\section{II.2.a Les $h$-longueurs sur $\widetilde{\text { Tess }}$}

Soit $\delta$ une tesselation rationnelle du disque de Poincaré (par exemple $\tau_{*}$ ), munie d'une arête orientée distinguée. Notons $\epsilon(\delta)$ l'ensemble des angles formés par les triangles de la tesselation $\delta$. Notons

$$
I: \mathbb{R}_{+}^{* \delta} \rightarrow \mathbb{R}_{+}^{* \epsilon(\delta)}
$$

l'application qui à $\Lambda \in \mathbb{R}_{+}^{*} \delta$ associe l'application $h$ définie par:

$$
h(E)=\frac{\Lambda(e)}{\sqrt{2} \Lambda(c) \Lambda(d)}
$$

où les arêtes $c, d, e$ et les angles $C, D, E$ sont représentés sur la figure suivante:

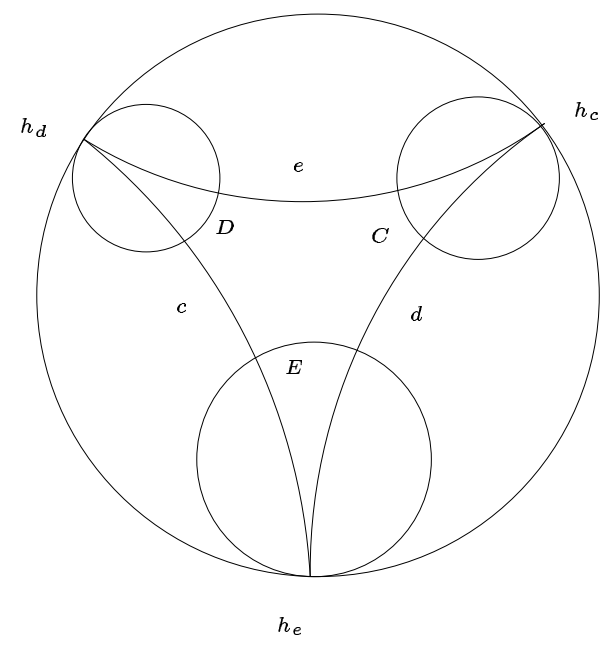

FIG. II.4 - Triangle geodésique décoré.

L'application $I$ est injective puisque l'on a l'égalité $h(C) h(D)=\frac{1}{2 \Lambda(e)^{2}}$. L'appellation h-longueur vient du lemme suivant:

Lemme II.3. Avec les notations de la figure précédente, $h(E)$ coïncide avec la moitié de la longueur hyperbolique du segment horocyclique sur $h_{e}$ délimité par les arêtes c et $d$.

La démonstration de ce lemme se trouve dans [Pen1], par exemple. Nous ne décrirons ni $\widetilde{T e s s}$, ni $\mathcal{G} r$ dans ce nouveau système de coordonnées. Remarquons simplement que pour un tel système de coordonnées associé à la tesselation de Farey et restreint à $\mathcal{G} r$, les coordonnées sont égales à $\frac{1}{2}$ sauf pour un nombre fini de termes. 


\section{II.2.b Longueur horocyclique}

Rappelons que $\widehat{\mathcal{C}_{n}}$ est l'espace des $(n+1)$-bracelets et $\mathcal{C}_{n}$ son normalisé défini à la section I.3.b. Soit $b=\left(h_{i}\right)_{i \in<1 ; n+1>} \in \widehat{\mathcal{C}_{n}}$.

Définition II.2.1. (Coordonnées horocycliques)

Soit $l_{i}=l_{i}(b)$ la longueur hyperbolique du segment de $h_{i}$ délimité par les arêtes $f_{i-1}=\left(x_{i-1} ; x_{i}\right)$ et $f_{i}=\left(x_{i} ; x_{i+1}\right) . l_{i}$ s'appelle la i-ème longueur horocyclique du bracelet $b$.

Remarquons que les fonctions $l_{i}$ sont invariantes par $P S L_{2} \mathbb{R}$. On a une proposition clef, portant sur les 3-bracelets.

Proposition II.2. Si $b=\left(h_{i}\right)_{i \in<1 ; 3>}$ est un bracelet constitué de trois horocycles, alors ses longueurs horocycliques sont toutes égales à 1 .

Preuve. - En effet, on peut supposer, quitte à appliquer une isométrie à $b$, se placer dans le cas où le point-base de $h_{1}\left(\right.$ resp. $\left.h_{2}, h_{3}\right)$ est $\frac{0}{1}$ (resp. $\left.\frac{1}{1}, \frac{1}{0}\right)$. On calcule alors la longueur horocyclique $l_{3}(b)$ :

$$
l_{3}(b)=\int_{I} \frac{d x}{y}=\int_{0}^{1} d x=1 \text { où } I=\{1 j+x, x \in[0 ; 1]\}
$$

Par 3-transitivité de $P S L_{2} \mathbb{R}$, il en est de même pour les deux autres longueurs horocycliques de $b$.

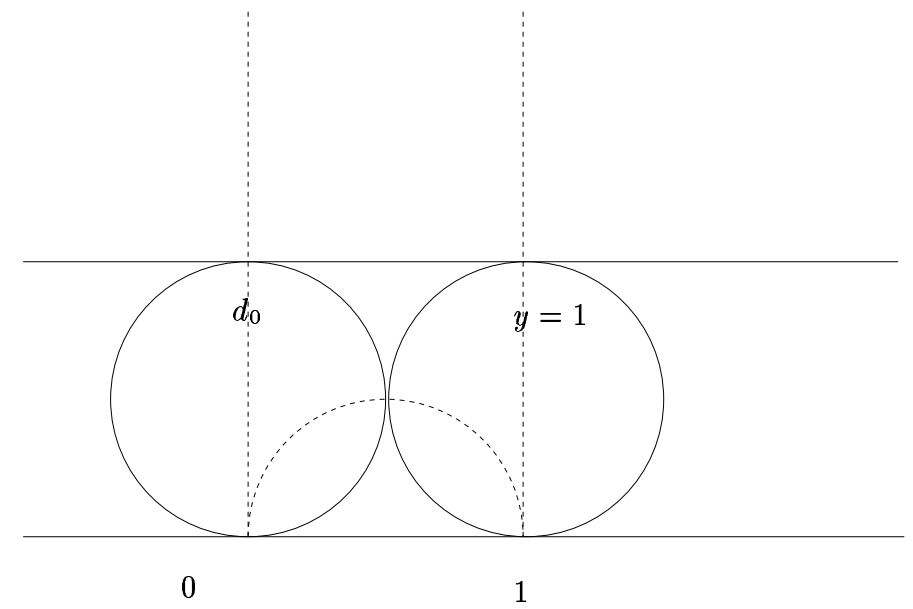

FIG. II.5 - Un triplet d'horocycles tangents deux à deux.

On remarque que la définition II.2.1 n'a plus de sens pour des collections d'horocycles quelconques. En effet, elle requiert que deux horocycles consécutifs de la collection soient tangents.

Pour obtenir les longueurs horocycliques en fonctions des lambda-longueurs, on normalise ces dernières. Pour $i \in<3, n>$, notons $e_{i}$ la géodesique d'extrémités $x_{1}$ et 
$x_{i}$ et $\sigma_{i}$ la lambda-longueur de $b$ le long de $e_{i}$ divisé par $\sqrt{2}$. Voici les formules reliant les coordonnées horocycliques aux coordonnées log-lambda.

Lemme II.4. 1. Si $n=3$, on a $l_{1}=l_{3}=\frac{2}{\sigma_{3}}$ et $l_{2}=l_{4}=\sigma_{3}$.

2. Puis dans le cas général:

$$
\begin{aligned}
& -l_{1}=\frac{1}{\sigma_{3}}+\sum_{j=2}^{n-2} \frac{1}{\sigma_{j+1} \sigma_{j+2}}+\frac{1}{\sigma_{n}} \\
& -l_{2}=\sigma_{3} \\
& -l_{3}=\frac{1+\sigma_{4}}{\sigma_{3}} \\
& -l_{i}=\frac{\sigma_{i-1}+\sigma_{i+1}}{\sigma_{i}} \forall i \in<4, n-1> \\
& -l_{n}=\frac{1+\sigma_{n-1}}{\sigma_{n}} \\
& -l_{n+1}=\sigma_{n}
\end{aligned}
$$

Preuve. - On traite directement le cas général. Soit $b \in \mathcal{C}_{n}$ et $P$ le $(n+1)$-gone hyperbolique dont l'ensemble des sommets correspond à $b k(b)$, (ensemble des pointsbase de $b$ ). Les géodésiques $e_{i}$ décomposent $P$ en $n-1$ triangles $T_{1}, \cdots, T_{n-1}$. Cela donne une triangulation idéale de $P$, qui décompose chaque horocycle de la façon suivante. $\forall(i, j) \in<1, n+1>\times<1, n-1>$, on note $l_{i}^{j}$ la longueur hyperbolique du segment $h_{i} \cap T_{j}$, de sorte que $l_{i}=\sum_{j=1}^{k_{i}} l_{i}^{j}$. Le lemme II.3 appliqué à chaque triangle $T_{j}$ donne:

$-T_{1}: l_{1}^{1}=\frac{1}{\sigma_{3}}, l_{2}^{1}=\sigma_{3}, l_{3}^{1}=\frac{1}{\sigma_{3}}$

$-T_{j}: l_{1}^{j}=\frac{1}{\sigma_{j+1} \sigma_{j+2}}, l_{j+1}^{j}=\frac{\sigma_{j+2}}{\sigma_{j+1}}, l_{j+2}^{j}=\frac{\sigma_{j+1}}{\sigma_{j+2}}$

$-T_{n-1}: l_{1}^{n-1}=\frac{1}{\sigma_{n}}, l_{n}^{n-1}=\frac{1}{\sigma_{n}}, l_{n+1}^{n-1}=\sigma_{n}$

et les formules demandées en découlent par sommations.

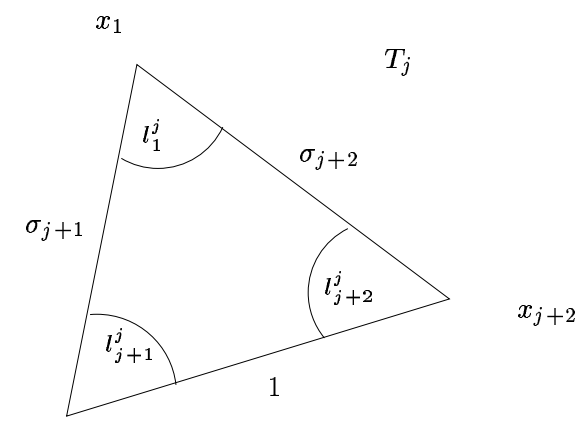

$x_{j+1}$

FIG. II.6 - Triangle $T_{j}$.

Notons $r_{n}$ l'application suivante:

$$
\begin{array}{rlrl}
r_{n}: & \mathbb{R}_{+}^{* n-2} & \rightarrow & \mathbb{R}_{+}^{*} n+1 \\
\left(\sigma_{3}, \cdots, \sigma_{n}\right) & \mapsto \quad\left(\frac{1}{\sigma_{3}}+\sum_{j=2}^{n-2} \frac{1}{\sigma_{j+1} \sigma_{j+2}}+\frac{1}{\sigma_{n}}, \sigma_{3}, \frac{1+\sigma_{4}}{\sigma_{3}}, \cdot, \frac{\sigma_{i-1}+\sigma_{i+1}}{\sigma_{i}}, \cdot \frac{1+\sigma_{n-1}}{\sigma_{n}}, \sigma_{n}\right)
\end{array}
$$

On en déduit la proposition suivante: 
Proposition II.3. L'application:

$$
\begin{array}{rlc}
p_{n}: \mathcal{C}_{n} & \rightarrow & \mathbb{R}_{+}^{* n+1} \\
b & \mapsto & \left(l_{1}(b), \cdots, l_{n+1}(b)\right)
\end{array}
$$

est un plongement dont l'image est paramétrée par $r_{n}\left(\mathbb{R}_{+}^{*}{ }^{n-2}\right)$.

Preuve. - La démonstration du théorème II.2 s'adapte sans difficulté (en déformant successivement le long des arêtes $e_{i}=\left(x_{1} ; x_{i}\right)$ pour $\left.i \in<3 ; n>\right)$ pour montrer que l'application

$$
\sigma: b \in \mathcal{C}_{n} \mapsto\left(\sigma_{i}(b)\right)_{i \in<3 ; n>} \in \mathbb{R}_{+}^{* n-2}
$$

est un homéomorphisme. Il suffit donc de montrer que l'application:

$$
r_{n}: \begin{array}{ccc}
\mathbb{R}_{+}^{* n-2} & \rightarrow & \mathbb{R}_{+}^{* n+1} \\
\left(\sigma_{3}, \cdots, \sigma_{n}\right) & \mapsto & \left(l_{1}, \cdots, l_{n+1}\right)
\end{array}
$$

est un homéomorphisme sur son image.

L'application $r_{n}$ est de classe $\mathcal{C}^{1}$, d'après les formules du lemme II.4. Elle est injective, puisque $\sigma_{3}$ est uniquement déterminé par $l_{2}$, puis $\sigma_{4}$ par $l_{3}$ et $l_{2}=\sigma_{3}$, etc.

C'est de plus une immersion. En effet, quitte à permuter cycliquement les coordonnées au but, par l'application

$$
\left(l_{1}, \cdots, l_{n+1}\right) \mapsto\left(l_{2}, \cdots, l_{n+1}, l_{1}\right)
$$

un calcul rapide montre que sa Jacobienne est constituée d'un bloc $(n-2) \times(n-2)$ triangulaire supérieur dont les éléments diagonaux sont, $1, \sigma_{3}^{-1}, \ldots, \sigma_{n-1}^{-1}$, et d'un bloc $(n-2) \times 3$.

Il reste à montrer que l'application $p_{n}$ est propre, c'est à dire que l'image réciproque d'un compact est un compact. Soit $K$ un compact de l'espace d'arrivée et $L$ sa préimage. Supposons que $L$ n'est pas compact. Il existe donc une suite définie par $\sigma^{k}=\left(\sigma_{i}^{k}\right)_{i \in<1 ; n>}$ dans $K$ dont l'image par $r_{n}$ est non bornée, c'est à dire qu'il n'existe pas de constante $A>1$ telle que la suite soit incluse dans le $(n-2)$-cube $\left[A^{-1} ; A\right]^{n-2}$. On va montrer que la suite image $r_{n}\left(\sigma^{k}\right)$ est non bornée (dans le sens précédent), contredisant ainsi la compacité de $K$. Soit $i_{0} \in<3, n>$ le plus petit entier tel que la suite réelle $\left(\sigma_{i}^{k}\right)_{k \in \mathbb{N}}$ soit non bornée.

Deux cas se présentent quitte à considérer une sous-suite de $\left(\sigma^{k}\right)_{k \in \mathbb{N}}$ :

- Cas 1: $\lim _{k \rightarrow \infty} \sigma_{i_{0}}^{k}=0$

Si $i_{0}=3$, alors la suite $l_{2}^{k}$ tend vers 0 , ce qui implique que $r_{n}\left(\sigma^{k}\right)$ n'est pas bornée dans $\mathbb{R}_{+}^{*}{ }^{n+1}$. Si $i_{0}>3$, considérons la suite $l_{i_{0}}^{k}=\frac{\sigma_{i_{0}-1}^{k}+\sigma_{i_{0}+1}^{k}}{\sigma_{i_{0}}^{k}}$. Puisque $\sigma_{i_{0}+1}^{k}>0$ pour tout $k$, on a la minoration $l_{i_{0}}^{k}>\frac{\sigma_{i_{0}-1}^{k}}{\sigma_{i_{0}}^{k}}$, ce qui entraine que $l_{i_{0}}^{k}$ tend vers $+\infty$, car le numérateur est minoré par une constante positive et le dénominateur tend vers 0 par hypothèse sur $i_{0}$. On en déduit que la suite $r_{n}\left(\sigma^{k}\right)$ n'est pas bornée.

- Cas 2: $\lim _{k \rightarrow \infty} \sigma_{i_{0}}^{k}=+\infty$ 
Si $i_{0}=3$, alors la suite image est non bornée puisque $l_{2}^{k}=\sigma_{3}^{k}$. Si $i_{0}>3$, considérons cette fois-ci la suite $l_{i_{0}-1}^{k}=\frac{\sigma_{i_{0}-2}^{k}+\sigma_{i_{0}}^{k}}{\sigma_{i_{0}-1}^{k}}$. Celle-ci tend vers $+\infty$ puisque les suites $\sigma_{i_{0}-2}^{k}$ et $\sigma_{i_{0}-1}^{k}$ sont majorées par hypothése sur $i_{0}$. On en déduit que la suite $r_{n}\left(\sigma^{k}\right)$ n'est pas bornée, ce qui montre que $r_{n}$ est propre.

Finalement l'application $r_{n}$ est une immersion injective et propre. C'est donc un homéomorphisme sur son image.

- Remarque: Les deux derniers résultats apparaissaient déjà dans l'appendice de [Gre3], mais sans démonstration. La méthode des log-lambda coordonnées nous a permis d'en donner des preuves transparentes.

Considérons pour $n \geq 1$, l'application suivante:

$$
\begin{array}{rlc}
q_{n}: \mathcal{G} r_{n} & \rightarrow & \left(\mathbb{R}_{+}^{*}\right)^{S_{n}} \\
f & \mapsto\left(l_{x}\left(f \cdot b_{n}^{F}\right)\right)_{x \in S_{n}}
\end{array}
$$

où $b_{n}^{F}$ est le bracelet de Ford basé en $S_{n}$ et $l_{x}\left(f \cdot b_{n}^{F}\right)$ la longueur horocyclique de $f \cdot b_{n}^{F}$ au point-base $f(x)$.

Munissons l'espace $\left(\mathbb{R}_{+}^{*}\right)^{S_{n}}$ de la métrique supérieure:

$$
d_{n}^{b}\left(l, l^{\prime}\right)=\sup _{x \in S_{n}}\left|l_{x}-l_{x}^{\prime}\right|
$$

La démonstration du lemme II.4 s'adapte mot pour mot pour montrer la:

Proposition II.4. L'application $q_{n}: \mathcal{G} r_{n} \rightarrow\left(\mathbb{R}_{+}^{*}\right)^{S_{n}}$ est un plongement.

Pour l'instant, les coordonnées horocycliques ne sont définies que sur les espaces filtrant $\mathcal{G} r$ et non sur $\mathcal{G} r$ tout entier, contrairement aux coordonnées log-lambda. Pour donner un système sur l'espace "total" $\mathcal{G} r$, il faut donc comprendre comment se traduisent les inclusions $i_{n}: \mathcal{G} r_{n} \subset \mathcal{G} r_{n+1}$ en terme des coordonnées horocycliques. Soit $n \geq 1$ et $j_{n}:\left(\mathbb{R}_{+}^{*}\right)^{S_{n}} \rightarrow\left(\mathbb{R}_{+}^{*}\right)^{S_{n+1}}$ l'application définie par:

$$
j_{n}\left(l_{1}, \cdots, l_{3 \cdot 2^{n-1}}\right)=\left(l_{1}+2,1, l_{2}+2,1, \cdots, 1, l_{3 \cdot 2^{n-1}}+2,1\right)
$$

Proposition II.5. Le diagramme:

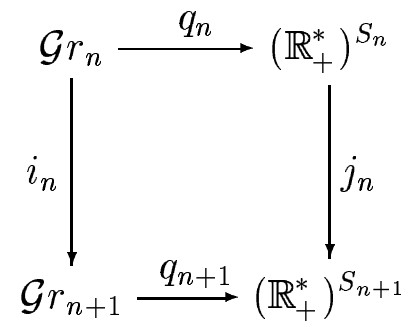

est commutatif. 
Preuve. - Il suffit d'appliquer la proposition II.2 pour chaque ajout de l'image par $f$ d'un horocycle de Ford basé en un point de $S_{n+1} \backslash S_{n}$.

Notons $\mathbb{R}_{+}^{*}(\widehat{\mathbb{Q}})$ la limite directe $\lim _{j_{n}}\left(\mathbb{R}_{+}^{*}\right)^{S_{n}}$. Grâce aux propositions II.4 et I.3, on obtient:

Corollaire II.3. L'application suivante:

$$
\begin{aligned}
& q: \quad \mathcal{G} r \rightarrow \mathbb{R}_{+}^{*}(\widehat{\mathbb{Q}}) \\
& f \in \mathcal{G} r_{n} \mapsto q_{n}(f)
\end{aligned}
$$

est définie et donne un homéomorphisme de $\mathcal{G} r$ sur son image.

\section{II.3 Une métrique sur $\mathcal{G} r$}

Remarquons que les applications $j_{n}:\left(\left(\mathbb{R}_{+}^{*}\right)^{S_{n}} ; d_{n}^{b}\right) \rightarrow\left(\left(\mathbb{R}_{+}^{*}\right)^{S_{n+1}} ; d_{n+1}^{b}\right)$ sont des isométries. Par conséquent, on peut munir $\mathbb{R}_{+}^{*}(\widehat{\mathbb{Q}})$ de la métrique $d^{b}$ définie par:

$$
d^{b}\left(l ; l^{\prime}\right)=d_{n}^{b}\left(l ; l^{\prime}\right)
$$

pour $\left(l, l^{\prime}\right) \in\left(\left(\mathbb{R}_{+}^{*}\right)^{S_{n}}\right)^{2}$.

La métrique pull-back de $d^{b}$ par l'application $q$ définit une nouvelle métrique sur l'espace $\mathcal{G} r$, appelée métrique horocyclique. On la note aussi $d^{b}$. Par définition, si $f$ et $g$ sont des éléments de $\mathcal{G} r_{n}$, on a $d^{b}(f ; g)=d_{n}^{b}\left(q_{n}(f) ; q_{n}(g)\right)$.

\section{II.4 Quelques questions de topologie}

Cette annexe traite succintement de problèmes de topologie qui sont apparus lors de la réalisation de ce travail. Nous allons citer six topologies apparaissant naturellement sur l'espace $\mathcal{G} r$ selon le contexte dans lequel on travaille. Nous essaierons ensuite de les comparer. Les résultats obtenus sont très partiels. Cependant, à l'aide de suites bien choisies, nous sommes en mesure de distinguer la plupart de ces topologies. Nous définissons ces topologies, en suivant notre étude sur l'espace $\mathcal{G} r$.

- La topologie en classe $\mathcal{C}^{1}:$ L'espace $\mathcal{G} r$ a été défini comme un sous-espace de l'ensemble des difféomorphismes de classe $\mathcal{C}^{1}$. Il est donc naturel de lui associer la métrique suivante:

$$
d_{1}(f ; g)=\operatorname{Max}\left\{\sup _{x \in \mathbb{S}^{1}} d(f(x) ; g(x)) ; \sup _{x \in \mathbb{S}^{1}}\left|f^{\prime}(x)-g^{\prime}(x)\right|\right\} \quad \forall(f, g) \in \mathcal{G} r^{2}
$$


- La topologie en classe $\mathcal{C}^{0}$ : On pourrait également le munir de la topologie $\mathcal{C}^{0}$ métrisée par:

$$
d_{0}(f ; g)=\sup _{x \in \mathbb{S}^{1}} d(f(x) ; g(x)) \quad \forall(f, g) \in \mathcal{G} r^{2}
$$

Celle-ci est moins fine que la topologie $\mathcal{C}^{1}$ sur l'espace des difféomorphismes de classe $\mathcal{C}^{1}$. Une question se pose tout de même. Qu'en est-il en restriction à l'espace $\mathcal{G} r$. En effet, la rigidité des éléments de $\mathcal{G} r$, pourrait entrainer que la convergence en classe $\mathcal{C}^{0}$ entraine celle en classe $\mathcal{C}^{1}$ (comme dans le cas des fonctions holomorphes où la convergence uniforme sur tout compact entraine la convergence uniforme sur tout compact de toutes les dérivées).

- La topologie faible: Par ailleurs, dans la définition I.2.3, nous avons filtré l'espace $\overline{\mathcal{G} r}$, et l'avons muni de la topologie faible. Notons $\mathcal{F}$ cette topologie. C'est la plus fine des topologies que l'on a définies sur $\mathcal{G} r$. En particulier, cette topologie vérifie la propriété suivante qui découle de la définition. Si $f \in \mathcal{G} r_{n}$ et $\left(f_{p}\right)_{p \geq 0}$ une suite de $\mathcal{G} r$ convergente vers $f$ pour la topologie faible, alors il existe $p_{0}$ tel que $\forall p \geq p_{0}$, on a $f_{p} \in \mathcal{G} r_{n}$ et la suite $\left(f_{p}\right)_{p \geq p_{0}}$ converge vers $f$ dans $\mathcal{G} r_{n}$ muni de sa topologie.

- Les métriques $d^{\Lambda}$ et $d^{b}$ : Dans la seconde partie, sont apparus deux systèmes de coordonnées sur l'espace $\mathcal{G} r$. Plus précisément, nous avons construit les deux applications suivantes qui sont injectives:

$$
\Lambda: \mathcal{G} r \rightarrow\left(\mathbb{R}_{+}^{*}\right)_{\sqrt{2}}^{\tau_{*}} \text { et } q: \mathcal{G} r \rightarrow \mathbb{R}_{+}^{*}(\widehat{\mathbb{Q}})
$$

Le pull-back de la métrique supérieure (resp. $\left.d^{b}\right) \operatorname{sur}\left(\mathbb{R}_{+}^{*}\right)_{\sqrt{2}}^{\tau_{*}}\left(\operatorname{resp} . \mathbb{R}_{+}^{*}(\widehat{\mathbb{Q}})\right.$ ) définit une nouvelle métrique notée $d^{\Lambda}$ (resp. $d^{b}$ ) sur l'espace $\mathcal{G} r$. Ces deux métriques définissent alors deux nouvelles topologies sur $\mathcal{G} r$.

- La métrique de Teichmüller: Enfin, l'espace $\mathcal{G} r$ est également un sous-espace de l'espace de Teichmüller universel de Bers $T(1)=P S L_{2} \mathbb{R} \backslash H o m e o_{q s}^{+}\left(\mathbb{S}^{1}\right)$ des homéomorphismes quasi-symétriques du cercle. Cet espace est muni de la métrique de Teichmüller $d_{\mathcal{T}}$ (voir [Gar-Lak] par exemple).

\section{II.4.a Les hyperboliques généralisés}

Pour distinguer certaines de ces topologies, nous construisons des suites dans l'espace $\mathcal{G} r$ à l'aide des éléments hyperboliques généralisés définis à la section I.2.b.

Soit $n \geq 1$. On note $S_{n}=\left(u_{i}^{n}\right)_{i \in<1 ; 3 \cdot 2^{n-1}>}$ de sorte que $u_{1}^{n}=0$ et que $u_{i}^{n}<u_{j}^{n}$ si $i<j$. Considérons l'élément hyperbolique généralisé $\theta_{n}^{t} \in \mathcal{G} r_{n}$ pour $n \geq 2$ dont la restriction à l'intervalle $\left[u_{i}^{n} ; u_{i+1}^{n}\right]$ coïncide avec la transformation hyperbolique d'axe $\left(u_{i}^{n} ; u_{i+1}^{n}\right)$ et dont la dérivée au point $u_{i}^{n}$ est égale à $t$ si $i$ est impair et $\frac{1}{t}$ si $i$ est pair.

L'élément $\theta_{n}^{t}$ vérifie les propriétés suivantes qui découlent immédiatement de la définition.

1. $b k\left(\theta_{n}^{t}\right)=S_{n}$. 
2. $\forall x \in S_{n-1}, \theta^{\prime t}(x)=t$.

3. $\frac{1}{t} \leq \theta_{n}^{\prime t}(x) \leq t$.

4. Pour toute suite de nombres réels $\left(t_{n}\right)_{n \in \mathbb{N}}$, la suite $\theta_{n}^{t_{n}}$ converge uniformément vers l'application identité du cercle.

Les trois premiers points découlent de la définition de $\theta_{n}^{t}$. Le dernier point provient $\mathrm{du}$ fait suivant: si $x \in\left[u_{i}^{n} ; u_{i+1}^{n}\right]$, alors il en est de même pour $\theta_{n}^{t_{n}}(x)$. Ainsi, pour la distance sphérique sur le cercle, on a $d_{\mathbb{S} 1}\left(\theta_{n}^{t_{n}}(x) ; x\right) \leq d_{\mathbb{S} 1}\left(u_{i+1}^{n} ; u_{i}^{n}\right)=\frac{1}{3 \cdot 2^{n}}$. On a donc $d_{0}\left(\theta_{n}^{t_{n}} ; i d\right)=\frac{1}{3 \cdot 2^{n}}$.

\section{II.4.b Leurs coordonnées}

Nous donnons les formules des coordonnées (log-lambda et horocycliques) des éléments construits ci-dessus.

\section{Coordonnées log-lambda}

Pour tout $n \geq 1$ et $t>0$, on a:

$$
\Lambda\left(\theta_{n}^{t}\right)(e)=\sqrt{2} \text { si } e \notin\left(\tau_{*}\right)_{n} \text { et } \Lambda\left(\theta_{n}^{t}\right)(e)=\frac{\sqrt{2}}{t} \text { si } e \in\left(\tau_{*}\right)_{n}
$$

\section{Coordonnées horocycliques}

Ici, les coordonnées dépendent du rang de la filtration sur lequel on se trouve. On obtient les coordonnées suivantes:

$$
\begin{gathered}
q_{2}(i d)=(3,1,3,1,3,1) \\
q_{3}(i d)=(5,1,3,1,5,1,3,1,5,1,3,1)
\end{gathered}
$$

puis au rang $n$ :

$$
q_{n}(i d)=(2 n-1,1,2 n-3,1, \cdots, 5,1,3,1)
$$

Pour les hyperboliques généralisés, nous avons:

$$
\begin{gathered}
q_{2}\left(\theta_{2}^{t}\right)=\left(3 t, \frac{1}{t}, 3 t, \frac{1}{t}, 3 t, \frac{1}{t}\right) \\
q_{3}\left(\theta_{3}^{t}\right)=\left(5 t, \frac{1}{t}, 3 t, \frac{1}{t}, 5 t, \frac{1}{t}, 3 t, \frac{1}{t}, 5 t, \frac{1}{t}, 3 t, \frac{1}{t}\right)
\end{gathered}
$$

puis au rang $n$ :

$$
q_{n}\left(\theta_{n}^{t}\right)=\left((2 n-1) t, \frac{1}{t},(2 n-3) t, \frac{1}{t}, \cdots, \frac{1}{t}, 5 t, \frac{1}{t}, 3 t, \frac{1}{t}\right)
$$




\section{II.4.c Calcul des distances}

On obtient ainsi la distance de $\theta_{n}^{t}$ à l'identité dans chaque système de coordonnées. Posons $d^{\Lambda}(f ; g)=\sup _{e \in \tau_{*}}|\Lambda(f)(e)-\Lambda(g)(e)|$.

$$
\begin{gathered}
d^{\Lambda}\left(\theta_{n}^{t} ; i d\right)=\sqrt{2}\left|1-\frac{1}{t}\right| \\
d^{b}\left(\theta_{n}^{t} ; i d\right)=(2 n-1)|t-1| \\
d_{0}\left(\theta_{n}^{t} ; i d\right)=\frac{1}{3 \cdot 2^{n}}
\end{gathered}
$$

et pour finir:

$$
d_{1}\left(\theta_{n}^{t} ; i d\right)=\operatorname{Max}\left\{\frac{1}{3 \cdot 2^{n}} ;|t-1| ;\left|\frac{1}{t}-1\right|\right\}
$$

\section{II.4.d Les convergences}

Définissons la suite $\left(u_{n}=\theta_{n}^{t_{n}}\right)_{n \geq 2}$ pour une suite à termes strictement positifs $\left(t_{n}\right)_{n \geq 2}$. Nous donnons des critères de convergence de la suite $\left(u_{n}\right)_{n \geq 2}$ en fonction du comportement de la suite $\left(t_{n}\right)_{n \geq 2}$.

Pour toute suite $\left(t_{n}\right)_{n \geq 2}$, la suite $\left(u_{n}\right)_{n \geq 2}$ converge vers l'identité pour la métrique $d_{0}$.

La suite $\left(u_{n}\right)_{n \geq 2}$ converge vers l'identité pour la métrique $d_{1}$ si et seulement si la suite $\left(t_{n}\right)_{n \geq 2}$ tend vers 1 .

La suite $\left(u_{n}\right)_{n \geq 2}$ converge vers l'identité pour la métrique $d^{b}$ si et seulement si $\left((2 n-1)\left(t_{n}^{2}-1\right)\right)_{n \geq 2}$ tend vers 0 .

La suite $\left(u_{n}\right)_{n \geq 2}$ converge vers l'identité pour la métrique $d^{\Lambda}$ si et seulement si $\left(t_{n}\right)_{n \geq 2}$ tend vers 1 .

Grace à ces critères de convergences, on en déduit que les topologies des métriques $d_{0}, d_{1}$ et $d^{b}$ sont deux à deux distinctes. Il en est de même pour les métriques $d_{0}, d^{\Lambda}$ et $d^{b}$.

Par contre, ces exemples ne distinguent pas les topologies des métriques $d^{\Lambda}$ et $d_{1}$. Pour ces deux métriques, nous avons le résultat suivant:

Proposition II.6. L'application identité de $\mathcal{G} r$ est continue pour la métrique $d_{1}$ à la source et la métrique $d^{\Lambda}$ au but.

Preuve. - Avant de commencer la preuve, rappelons deux résultats permettant d'exprimer les lambda-longueurs en fonction des dérivées. Ces formules sont calculées dans [Mal-Pen].

Soient $h_{0}$ (resp. $\left.h_{1}\right)$ un horocycle de $\mathbb{H}^{2}$ basé en $x_{0} \in \mathbb{R}$ (resp. $x_{1} \in \mathbb{R}$ ) et de diamètre euclidien $d_{0}$ (resp. $d_{1}$ ). Le lemme A.1. dans [Mal-Pen] donne la lambda-longueur de la décoration $h=\left(h_{0} ; h_{1}\right)$ le long de l'arête $e=\left(x_{0} ; x_{1}\right)$ :

$$
\Lambda(h ; e)=\sqrt{\frac{2}{d_{0} d_{1}}}\left|x_{0}-x_{1}\right|
$$


Considérons $h \in P S L_{2} \mathbb{R}$ et $h_{0}$ basé en un point $x_{0} \in \mathbb{R}$, tels que $h\left(x_{0}\right)$ soit dans $\mathbb{R}$. D'après le lemme A.2. du même article, l'horocycle image $h\left(h_{0}\right)$, basé en $h\left(x_{0}\right)$ a pour diamêtre euclidien $\left|h^{\prime}\left(x_{0}\right)\right| d_{0}$.

Soit $f \in C P P$ tel que $f(\infty)=\infty$ et $e=(x ; y)$ une arête dont les extrémités sont dans $\mathbb{R}$. Les deux résultats combinés, on obtient la formule suivante:

$$
\frac{\Lambda(f(h) ; f(e))}{\Lambda(h ; e)}=\frac{\left|f\left(x_{0}\right)-f\left(x_{1}\right)\right|}{\left|x_{0}-x_{1}\right|} \sqrt{\frac{1}{f^{\prime}\left(x_{0}\right) f^{\prime}\left(x_{1}\right)}}
$$

Venons-en à la démonstration. Fixons $f \in \mathcal{G} r$ et $\epsilon>0$. Pour simplifier le calcul, on se place sur l'intervalle $[0 ; 1]$. On pose $\tau_{*}^{0}=\left\{e=(x ; y) \in \tau_{*} \mid(x, y) \in[0 ; 1]^{2}\right\}$. On va montrer qu'il existe $\alpha>0$ tel que:

$$
d_{1}(f ; g)<\alpha \Longrightarrow \sup _{e \in \tau_{*}^{0}}\left|\frac{\Lambda(f)(e)-\Lambda(g)(e)}{\sqrt{2}}\right|<\epsilon \quad \forall g \in \mathcal{G} r
$$

Soit $e=(p ; q) \in \tau_{*}^{0}$ avec $p<q$. D'après la formule II.3, appliquée à l'arête $e$ décoré par le bracelet de Ford, on a:

$$
\frac{\Lambda(f)(e)-\Lambda(g)(e)}{\sqrt{2}}=\left[\frac{f(q)-f(p)}{q-p}\right] \sqrt{\frac{1}{f^{\prime}(p) f^{\prime}(q)}}-\left[\frac{g(q)-g(p)}{q-p}\right] \sqrt{\frac{1}{g^{\prime}(p) g^{\prime}(q)}}
$$

Puisque $f$ et $g$ sont de classe $\mathcal{C}^{1}$, il existe deux réels $x_{p, q}^{f}$ et $x_{p, q}^{g}$ dans l'intervalle $[p ; q]$ tels que $f^{\prime}\left(x_{p, q}^{f}\right)=\frac{f(q)-f(p)}{q-p}$ et $g^{\prime}\left(x_{p, q}^{g}\right)=\frac{g(q)-g(p)}{q-p}$. Ce qui entraine, couplé à une inégalité triangulaire, l'inégalité:

$$
\left|\frac{\Lambda(f)(e)-\Lambda(g)(e)}{\sqrt{2}}\right| \leq\left|\frac{f^{\prime}\left(x_{p, q}^{f}\right)}{\sqrt{f^{\prime}(p) f^{\prime}(q)}}-\frac{f^{\prime}\left(x_{p, q}^{g}\right)}{\sqrt{f^{\prime}(p) f^{\prime}(q)}}\right|+\left|\frac{f^{\prime}\left(x_{p, q}^{g}\right)}{\sqrt{f^{\prime}(p) f^{\prime}(q)}}-\frac{g^{\prime}\left(x_{p, q}^{g}\right)}{\sqrt{g^{\prime}(p) g^{\prime}(q)}}\right|
$$

Par uniforme continuité de $f^{\prime}$ sur $[0 ; 1]$ et comme $\left|x_{p, q}^{g}-x_{p, q}^{f}\right|<q-p$, on peut trouver un sous-ensemble fini $A_{f}$ de $\tau_{*}^{0}$ tel que:

$$
\forall e \in \tau_{*}^{0} \backslash A_{f} \quad\left|f^{\prime}\left(x_{p, q}^{g}\right)-f^{\prime}\left(x_{p, q}^{f}\right)\right|<\frac{\epsilon}{2 \inf _{x \in[0 ; 1]} f^{\prime}(x)}
$$

(la dérivée de $f$ ne s'annulant pas). Puisque $f^{\prime}(p)$ et $f^{\prime}(q)$ sont supérieurs à cette borne inférieure, on obtient la majoration du premier terme à droite de l'inégalité II.5:

$$
\left|\frac{f^{\prime}\left(x_{p, q}^{f}\right)}{\sqrt{f^{\prime}(p) f^{\prime}(q)}}-\frac{f^{\prime}\left(x_{p, q}^{g}\right)}{\sqrt{f^{\prime}(p) f^{\prime}(q)}}\right| \leq \frac{\epsilon}{2} \quad \forall e \in \tau_{*}^{0} \backslash A_{f}
$$

Concernant le second membre, comme la dérivée de $f$ est minorée par une constante strictement positive, on peut trouver $\alpha>0$ tel que $\forall(x, y, z, f) \in[0 ; 1]^{3} \times B_{d_{1}}(f ; \alpha)$, on a la majoration:

$$
\left|\frac{f^{\prime}(x)}{\sqrt{f^{\prime}(y) f^{\prime}(z)}}-\frac{g^{\prime}(x)}{\sqrt{g^{\prime}(y) g^{\prime}(z)}}\right| \leq \frac{\epsilon}{2}
$$


En substituant II.6 et II.7 (appliquée aux réels $x=x_{p, q}^{g}, y=p$ et $z=q$ ) dans l'inégalité II.5, on obtient:

$$
\left|\frac{\Lambda(f)(e)-\Lambda(g)(e)}{\sqrt{2}}\right|<\epsilon \quad \forall(g, e) \in B_{d_{1}}(f ; \alpha) \times \tau_{*}^{0} \backslash A_{f}
$$

Comme l'ensemble $A_{f}$ est fini, l'application $g \in \mathcal{G} r \mapsto(\Lambda(g))_{e \in A_{f}} \in\left(\mathbb{R}_{+}^{*}\right)^{\operatorname{card}\left(A_{f}\right)}$ est continue pour la norme $d_{1}$ à la source. On en déduit donc, quitte à diminuer $\alpha$, que l'inégalité II.8 est vérifiée pour tout élément de $\tau_{*}^{0}$, ce qui montre l'assertion II.4.

Le même raisonnement peut s'appliquer aux trois intervalles $[1 ; \infty],[\infty ;-1]$ et $[-1 ; 0]$. Enfin, il reste l'arête orientée $e_{0}=(0 ; \infty)$ seule arête de Farey dont les extrémités ne sont pas contenues dans un des quatre intervalles considérés. L'application $g \in \mathcal{G} r \mapsto \Lambda(g)\left(e_{0}\right) \in \mathbb{R}_{+}^{*}$ est continue. Finalement il existe $\alpha>0$ tel que pour tout $(e, g) \in \tau_{*} \times B_{d_{1}}(f ; \alpha)$, on a l'inégalité II.8, ce qui montre la proposition. 


\section{Chapitre III}

\section{Géométrie du groupe de Thompson}

Ce chapitre est consacré à l'étude de l'action du groupe de Thompson $T$ sur l'espace contractile $\mathcal{G} r$. Après avoir défini cette action (III.1), nous l'étudions dans le système des coordonnées log-lambda (III.2).

Les sections III.3 et III.4 traitent de l'étude des orbites dans les deux systèmes de coordonnées. L'action du groupe est discrète (proposition III.3). En comparaison à [Gre4], notre démonstration est nouvelle. Elle utilise les coordonnées log-lambda. On donnera une autre démonstration dans le cadre des coordonnées horocycliques. De plus, le système des coordonnées horocycliques fait apparaitre $T$ comme groupe d'isométries (proposition III.4). On déduit alors une propriété de discontinuité faible (proposition III.6) établie différemment dans [Gre4].

La section III.5 reprend l'étude des stabilisateurs de l'action, faite dans [Gre4], par une méthode plus directe, puisqu'elle évite de recourir au théorème de Denjoy sur le nombre de rotation et à un théorème de lissage du groupe $T$ [Ghy-Ser]. Puis, après une rapide discussion (III.6) sur l'analogie entre l'action de $T$ sur $\mathcal{G} r$ et celle du Mapping Class Group sur l'espace de Teichmüller d'une surface, nous obtenons la contractibilité faible de $\mathcal{G} r$ privé de l'orbite de l'identité (proposition III.8).

Nous terminons ce chapitre en donnant un classifiant du groupe $T$ (corollaire III.2). Ceci étend la méthode de [Gre4], dans lequel un classifiant de $F^{\prime}$ était exhibé (corollaire III.1).

\section{III.1 L'action du groupe de Thompson sur $\mathcal{G} r$ et $\mathcal{C} r$}

Avant de définir l'action du groupe de Thompson $T$ sur l'espace $\mathcal{G} r$, nous donnons le point-clef de l'étude de cette action.

Lemme III.1. Le bracelet de Ford $b^{F}=\left(h_{x}^{F}\right)_{x \in \widehat{\mathbb{Q}}}$ est globalement invariant par l'action de $T$ à gauche. En d'autres termes, on a:

$$
\gamma\left(h_{x}^{F}\right)=h_{\gamma(x)}^{F} \forall(x, \gamma) \in \widehat{\mathbb{Q}} \times T
$$


Preuve. - Puisque $P S L_{2} \mathbb{Z}$ fixe le bracelet de Ford, il en est de même pour $T=$ $P P S L_{2} \mathbb{Z}$.

Nous aurons besoin de connaitre l'ensemble des points de coupure et le décalage de la composé de deux homéomorphismes du cercle projectifs par morceaux.

Lemme III.2. $\forall(x, u, v) \in \mathbb{S}^{1} \times C P P^{2}$, on $a$ :

$$
\begin{array}{lcc}
b k(u \circ v) & \subset & v^{-1} b k(u) \cup b k(v) \\
D_{x}(u \circ v) & =\left(v_{x}^{r}\right)^{-1} \circ D_{v x} u \circ\left(v_{x}^{l}\right)
\end{array}
$$

La preuve est immédiate. En particulier, si $h \in P S L_{2} \mathbb{R}$, et $f \in C P P$, alors on a les égalités $b k(h \circ f)=b k(f)$ et $b k(f \circ h)=h^{-1}(b k(f))$ pour les points de coupure et les égalités $D_{x}(h \circ f)=D_{x} f$ et $D_{x}(f \circ h)=h^{-1} D_{h(x)} h$ pour les décalages.

Définition III.1.1. (Action de T)

Le groupe $T$ agit librement par composition à droite sur l'espace $\widehat{\mathcal{G} r}$ puisque:

$$
\forall(f, \gamma) \in \widehat{\mathcal{G r}} \times T, b k(f \circ \gamma) \subset b k(\gamma) \cup \gamma^{-1}(b k(f)) \subset \widehat{\mathbb{Q}}
$$

Cette action par composition passe alors en une action sur l'espace quotient $\mathcal{G} r$. De plus, elle transite par l'homéomorphisme $\psi^{-1}: \mathcal{C} r \rightarrow \mathcal{G} r$ (corollaire I.3) en une action sur $\mathcal{C} r$, provenant de l'action à gauche de $C P P \operatorname{sur} \mathcal{H}$ (corollaire I.1).

L'action étant définie dans le langage CPP, comment se traduit-elle dans les différents systèmes de coordonnées?

\section{III.2 L'action sur les coordonnées log-lambda}

Considérons $\Delta_{\mathbb{Q}}$, l'ensemble des géodésiques du disque de Poincaré dont les extrémités sont dans $\widehat{\mathbb{Q}}$. Avec les notations de la section II.1, on a $\Delta_{\mathbb{Q}}=\widehat{\mathbb{Q}}^{(1)}$. Le groupe de Thompson agit sur $\Delta_{\mathbb{Q}}$ puisqu'il fixe $\widehat{\mathbb{Q}}$. Plus précisément, cette action est donnée par la formule:

$$
\gamma \cdot(x ; y)=(\gamma(x) ; \gamma(y))
$$

Soit $f$ un élément de $\widehat{\mathcal{G r}}$. La collection d'horocycles $f \cdot b^{F}$ est portée par l'ensemble $f(\widehat{\mathbb{Q}})$. Si $e \in \Delta_{\mathbb{Q}}$, alors $f(e)$ est un élément de $f\left(\widehat{\mathbb{Q}}^{(1)}\right)$ et le nombre $\Lambda\left(f \cdot b^{F} ; f(e)\right)$ a été défini comme la lambda-longueur de la collection $f \cdot b^{F}$ le long de l'arête $f(e)$. Notons $\Lambda(f ; e)$ cette lambda-longueur. On a ainsi associé un paramètre sur $\widehat{\mathcal{G r}}$ à chaque élément de $\Delta_{\mathbb{Q}}$. Remarquons que ce paramètre est invariant par l'action à gauche de $P S L_{2} \mathbb{R}$.

Proposition III.1. Soit $f \in \widehat{\mathcal{G r}}, \gamma \in T$ et $e \in \Delta_{\mathbb{Q}}$. Alors on a l'égalité suivante des lambda-longueurs:

$$
\Lambda(f ; \gamma \cdot e)=\Lambda(f \circ \gamma ; e)
$$


Preuve. - Par définition, on a

$$
\begin{gathered}
\Lambda(f ; \gamma \cdot e)=\Lambda\left(f \cdot b^{F} ; f(\gamma \cdot e)\right) \\
\Lambda(f \circ \gamma ; e)=\Lambda\left((f \circ \gamma) \cdot b^{F} ; f \circ \gamma(e)\right)
\end{gathered}
$$

De plus, $f(\gamma \cdot e)=f \circ \gamma(e)$ et d'après le lemme III.1, on a $f \cdot b^{F}=f \circ \gamma \cdot b^{F}$, ce qui entraine l'égalité des membres de droite et la proposition.

Considérons à présent l'espace des tesselations rationnelles:

$$
T e s s_{\mathbb{Q}}^{\prime}=\left\{\tau \in T e s s^{\prime} \mid \tau^{(0)}=\widehat{\mathbb{Q}}\right\}
$$

Comme $\tau_{*}$, tout élément $\tau$ de $T e s s_{\mathbb{Q}}^{\prime}$ fournit un système de coordonnées sur $\mathcal{G} r$. On le note $\Lambda^{\tau}$. Il provient de l'application $P S L_{2} \mathbb{R}$-invariante:

$$
\Lambda^{\tau}: f \in \widehat{\mathcal{G r}} \mapsto \Lambda^{\tau}(f)=(\Lambda(f ; e))_{e \in \tau} \in \mathbb{R}_{+}^{* \tau}
$$

Par exemple, le système $\Lambda^{\tau_{*}}$ correspond exactement au système $\Lambda$ de la section II.1.b.

Le groupe de Thompson $T$ agit à gauche sur $T e s s_{\mathbb{Q}}^{\prime}$. Cette action correspond, en termes de coordonnées log-lambda, à des changements de coordonnées. Plus exactement:

Lemme III.3. Soit $f \in \mathcal{G} r, \gamma \in T$ et $e \in \tau_{*}$. On a les égalités suivantes des coordonnées log-lambda:

$$
\Lambda(f \cdot \gamma)(e)=\Lambda^{\tau_{*}}(f \cdot \gamma)(e)=\Lambda^{\gamma \cdot \tau_{*}}(f)(\gamma \cdot e)
$$

Preuve. - Soit $h \in P S L_{2} \mathbb{R}$ tel que $h \circ f \circ \gamma=f \cdot \gamma$. Puisque $\Lambda^{\tau_{*}}$ est $P S L_{2} \mathbb{R}$-invariante, on a:

$$
\Lambda(f \cdot \gamma)(e)=\Lambda^{\tau_{*}}(h \circ f \circ \gamma)(e)=\Lambda^{\tau_{*}}(f \circ \gamma)(e)
$$

Par ailleurs, d'après la proposition III.1

$$
\Lambda^{\tau_{*}}(f \circ \gamma)(e)=\Lambda(f \circ \gamma ; e)=\Lambda(f ; \gamma \cdot e)
$$

Le dernier terme est par définition $\Lambda^{\gamma \cdot \tau_{*}}(f)(\gamma \cdot e)$, ce qui termine la preuve.

Afin de comprendre l'intéret de cette observation, rappelons la formule de Ptolémée portant sur les coordonnées log-lambda. Soit $a, b, c, d$ les arêtes d'un quadrilatère idéal du disque de Poincaré $Q$, tel que $a$ et $c$ sont des cotés opposés. Notons $e$ et $f$ les deux diagonales de ce quadrilatère. Supposons le quadrilatère décoré par une collection d'horocycles notée $h$. Rappelons que $\Lambda(h ; x)$ est la lambda- longueur de $h$ le long de $x$, pour une arête dont les extrémités sont des sommets de $Q$.

Proposition III.2. (Formule de Ptolémée [Pen1]) On a l'égalité

$$
\Lambda(h ; e) \Lambda(h ; f)=\Lambda(h ; a) \Lambda(h ; c)+\Lambda(h ; b) \Lambda(h ; d)
$$


Muni de cette formule, nous pouvons calculer les coordonnées log-lambda de $f \cdot \gamma$ en fonction des coordonnées de $f$ lorsque $\gamma$ correspond à un petit nombre de mouvements élémentaires dans le modèle du groupe de Ptolémée (définition I.6.3).

Il vient une autre conséquence de l'invariance du bracelet de Ford par l'action du groupe $T$. Rappelons que $\widehat{\mathcal{B} r}$ est la limite directe des espaces de bracelets propres $\left(\widehat{\mathcal{B} r_{n}}\right)_{n \geq 1}$ introduite en I.3. Vu comme sous-espace de $\mathcal{G} r$, il s'identifie aux éléments $f \in \mathcal{G} r$ vérifiant la propriété:

$$
\forall(x ; y) \in \widehat{\mathbb{Q}}^{2} \mid f\left(h_{x}^{F}\right) \text { et } f\left(h_{y}^{F}\right) \text { ne s'intersectent pas transversalement. }
$$

Cette propriété est conservée par l'action par composition à gauche de $P S L_{2} \mathbb{R}$.

Lemme III.4. L'action du groupe $T$ se restreint aux sous-espace des bracelets propres $\mathcal{B} r$.

Preuve. - Soient $f \in \mathcal{B} r$ et $\gamma \in T$. Quelque soit $x \in \widehat{\mathbb{Q}}$, l'horocycle de la décoration de $(f \circ \gamma) \cdot \tilde{\tau}_{*}$, (basé en $\left.(f \circ \gamma)(x)\right)$ est exactement l'horocycle $f\left(h_{\gamma(x)}^{F}\right)\left(h_{\gamma(x)}^{F}\right.$ est l'horocycle de Ford basé en $\gamma(x))$. Ainsi, si $f \in \widehat{\mathcal{G} r}$ vérifie la propriété III.2, il en est de même pour $f \circ \gamma$. Finalement $f \cdot \gamma$ est un élément de $\mathcal{B} r$.

- Attention: Signalons que le groupe de Thompson n'a aucune raison d'agir en préservant les filtrations de $\mathcal{B} r$ et $\mathcal{G} r$ définies à partir de la filtration de $\widehat{\mathbb{Q}}$.

\section{III.3 Etude des orbites}

Nous montrons dans cette partie que les orbites de l'action de $T$ sur $\mathcal{G} r$ sont discrètes. On fixe dorénavant un élément $g \in \mathcal{G} r_{p}$ et on étudie son orbite.

Le raisonnement consiste à trouver des conditions vérifiées par les coordonnées log-lambda sur l'orbite de $g$ à partir des coordonnées de $g$, afin de montrer que les orbites sont discrètes. On commence par traiter le cas $g=i d$, afin d'illustrer l'idée de la démonstration. Notons $\left(\mathbb{N}_{+}^{*}\right)_{\sqrt{2}}^{\tau_{*}}$ le sous-espace de $\left(\mathbb{R}_{+}^{*}\right)_{\sqrt{2}}^{\tau_{*}}$ suivant:

$$
\left(\mathbb{N}_{+}^{*}\right)_{\sqrt{2}}^{\tau_{*}}=\left\{\Lambda \in\left(\mathbb{R}_{+}^{*}\right)_{\sqrt{2}}^{\tau_{*}} \mid \Lambda(e) \in \mathbb{N}^{*} \sqrt{2}\right\}
$$

C'est un sous-espace discret de $\mathcal{G} r$. Notons

$$
\left(\Delta_{\mathbb{Q}}\right)_{n}=\left\{e=(x ; y) \in \Delta_{\mathbb{Q}} \mid(x, y) \in S_{n}\right\}
$$

Lemme III.5. L'orbite de l'identité est contenue dans l'ensemble $\left(\mathbb{N}_{+}^{*}\right)_{\sqrt{2}}^{\tau_{*}}$.

Preuve. - On commence par montrer l'assertion suivante par récurrence sur l'indexation de la filtration de $\widehat{\mathbb{Q}}$.

$$
\mathcal{P}(n): \text { " } \forall e \in\left(\Delta_{\mathbb{Q}}\right)_{n} \text { on a } \Lambda\left(b^{F} ; e\right) \in \mathbb{N}^{*} \sqrt{2} \text { " }
$$


- Le rang 1: Par définition du bracelet de Ford, $\Lambda\left(b^{F} ; e\right)=\sqrt{2}$, si $e$ a ses extrémités dans $\overline{S_{1}}$.

- Du rang $n$ au rang $n+1$ : Supposons l'assertion vraie au rang $n$. Soit $e=(x ; y) \in$ $\left(\Delta_{\mathbb{Q}}\right)_{n+1}$. On utilise la formule de Ptolémée (III.1) dans la situation décrite par la figure III.1. Les points $a, b$ désignent les points de $S_{n}$, voisins immédiat dans $S_{n+1}$ du point $x \in S_{n+1}$. En particulier, le triangle $(a ; x ; b)$ est un triangle de Farey. On procède en deux cas:

$\star$ Cas 1: $x \in S_{n+1} \backslash S_{n}$ et $y \in S_{n}$ : Lorsque $y \in S_{n}$, l'égalité de Ptolémée donne

$$
\Lambda\left(b^{F} ; e\right) \Lambda\left(b^{F} ; f\right)=\Lambda\left(b^{F} ; e_{1}\right) \Lambda\left(b^{F} ; e_{3}\right)+\Lambda\left(b^{F} ; e_{2}\right) \Lambda\left(b^{F} ; e_{4}\right)
$$

Puisque $(a ; x ; b)$ est un triangle de Farey, les lambda longueurs $\Lambda\left(b^{F} ; f\right), \Lambda\left(b^{F} ; e_{1}\right)$ et $\Lambda\left(b^{F} ; e_{2}\right)$ sont égales à $\sqrt{2}$. Par hypothèse de récurrence, il existe deux entiers non nuls $n_{3}$ et $n_{4}$ tels que $\Lambda\left(b^{F} ; e_{i}\right)=n_{i} \sqrt{2}$ pour $i \in\{3 ; 4\}$. On obtient donc $\Lambda\left(b^{F} ; e\right)=$ $\left(n_{3}+n_{4}\right) \sqrt{2}$.

$\star$ Cas 2: $x \in S_{n+1} \backslash S_{n}$ et $y \in S_{n+1} \backslash S_{n}$ : Puisque $a$ et $b$ sont dans $S_{n}$, le cas précédent entraine l'existence de deux entiers positifs $n_{3}$ et $n_{4}$ tels que $\Lambda\left(b^{F} ; e_{3}\right)=n_{3} \sqrt{2}$ et $\Lambda\left(b^{F} ; e_{4}\right)=n_{4} \sqrt{2}$. La formule de Ptolémée donne encore $\Lambda\left(b^{F} ; e\right)=\left(n_{3}+n_{4}\right) \sqrt{2}$.

On a ainsi montré que pour tout $e \in \Delta_{\mathbb{Q}}$, on a $\Lambda\left(b^{F} ; e\right) \in \mathbb{N}^{*} \sqrt{2}$. Pour conclure le lemme, prenons $e \in \tau_{*}$ et $\gamma \in T$. Puisque $\gamma(e)$ est une arête rationnelle, on sait que $\Lambda\left(b^{F} ; \gamma(e)\right) \in \mathbb{N}^{*} \sqrt{2}$. D'après le lemme III.3, la quantité précédente est égale à $\Lambda(f \cdot \gamma)(e)$, ce qui termine la démonstration du lemme.
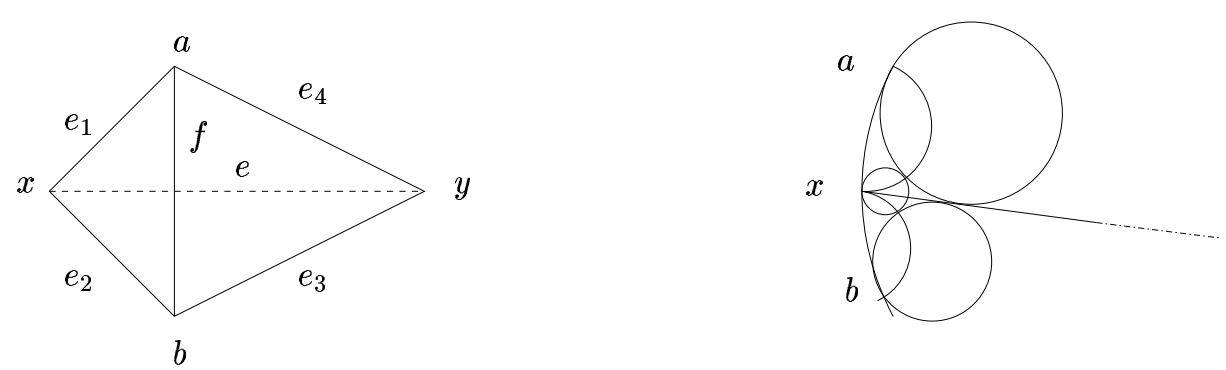

FIG. III.1 - Formule de Ptolémée.

Traitons à présent le cas général. Pour tout sous-ensemble fini $A$ de $\mathbb{R}_{+}^{*}$, tel que $A=\left\{a_{0}=\sqrt{2} ; a_{1} ; \cdots ; a_{k}\right\}$, on définit le sous-ensemble de $\mathbb{R}_{+}^{*}$ suivant

$$
N(A)=\left\{\sum_{i=0}^{k} n_{i} a_{i} \mid\left(n_{i}\right)_{i \in<0 ; k>} \in(\mathbb{N})^{k+1} \backslash\{0\}\right\}
$$

Remarquons que $N(A)$ est discret dans $\mathbb{R}_{+}^{*}$. A partir de $N(A)$, on définit également l'ensemble:

$$
\left(\mathbb{R}_{+}^{*}\right)_{A}^{\tau_{*}}=\left\{\Lambda \in\left(\mathbb{R}_{+}^{*}\right)_{\sqrt{2}}^{\tau_{*}} \mid \Lambda(e) \in N(A) \quad \forall e \in \tau_{*}\right\}
$$


Ce sous-ensemble de $\left(\mathbb{R}_{+}^{*}\right)_{\sqrt{2}}^{\tau_{*}}$ est stable pour l'addition et discret pour la topologie faible (mais aussi pour la métrique supérieure de l'espace des paramètres).

Attention: la notation $\left(\mathbb{R}_{+}^{*}\right)_{A}^{\tau_{*}}$ ne correspond pas à la notation $\left(\mathbb{R}_{+}^{*}\right)_{\sqrt{2}}^{\tau_{*}}$ avec $A=$ $\{\sqrt{2}\}$.

Proposition III.3. L'image de l'orbite de g par $\Lambda$ est inclue dans $\left(\mathbb{R}_{+}^{*}\right)_{A}^{\tau_{*}}$. En particulier $T$ agit sur $\mathcal{G} r$ avec orbites discrètes.

Preuve. - On utilise un argument similaire à la démonstration faite dans le cas de l'identité.

Supposons que $g \in \mathcal{G} r_{p}$. L'ensemble des géodésiques dont les sommets sont inclus dans $S_{p}$ est fini. On le note $\left\{e_{1} ; \cdots ; e_{k}\right\}$ et on définit $a_{k}$ comme étant la lambda-longueur de l'arête $g \cdot e_{k}$ relativement à la décoration de $g \cdot \tilde{\tau}_{*}$. On note $a_{0}=\sqrt{2}$.

On montre par récurrence l'assertion suivante pour $n \geq p$ :

$$
\mathcal{P}(n):^{\prime \prime} \forall e \in\left(\Delta_{\mathbb{Q}}\right)_{n} \Lambda\left(g\left(b^{F}\right) ; g(e)\right) \in N(A)^{\prime \prime}
$$

L'assertion est vraie au rang $n=p$, par définition des ensembles $\left\{a_{1} ; \cdots a_{k}\right\}$ et $N(A)$.

Supposons la vraie au rang $n \geq p$ et prenons $e=(x ; y)$ une arête dont les extrémités sont dans $S_{n+1}$. Deux cas se présentent:

- Cas 1: $x \in S_{n+1} \backslash S_{n}$ et $y \in S_{n}$ Reprenons la situation de la figure III.1. Puisque

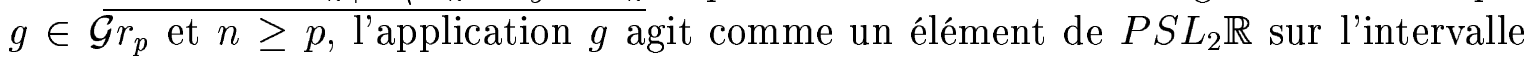
$[a ; b]$ contenant $x$. Ainsi, on a les égalités

$$
\Lambda\left(g\left(b^{F}\right) ; g(f)\right)=\Lambda\left(g\left(b^{F}\right) ; g\left(e_{1}\right)\right)=\Lambda\left(g\left(b^{F}\right) ; g\left(e_{2}\right)\right)=\sqrt{2}
$$

Puis, par hypothèse de récurrence, il existe deux suites d'entiers positifs $\left(n_{i}^{3}\right)_{i \in<0 ; k+1>}$ et $\left(n_{i}^{4}\right)_{i \in<0 ; k+1>}$ non tous nuls tels que:

$$
\Lambda\left(g\left(b^{F}\right) ; g\left(e_{3}\right)\right)=\sum_{i=0}^{k} n_{i}^{3} a_{i} \quad \text { et } \quad \Lambda\left(g\left(b^{F}\right) ; g\left(e_{4}\right)\right)=\sum_{i=0}^{k} n_{i}^{4} a_{i}
$$

La formule de Ptolémée entraine que $\Lambda\left(g\left(b^{F}\right) ; g(e)\right)=\sum_{i=0}^{k}\left(n_{i}^{3}+n_{i}^{4}\right) a_{i}$.

- Cas 2: $x \in S_{n+1} \backslash S_{n}$ et $y \in S_{n+1} \backslash S_{n}$ L'argument est le même que dans le cas de l'orbite de l'identité. Ceci termine la récurrence.

Finalement, pour toute arête $e \in \Delta_{\mathbb{Q}}$, on a $\Lambda\left(g\left(b^{F}\right) ; g(e)\right) \in N(A)$.

Soient $e \in \tau_{*}$ et $\gamma \in T$. D'après le lemme III.3 et par définition de $\Lambda^{\gamma \cdot \mathcal{\tau}_{*}}$, on a

$$
\Lambda(g \cdot \gamma)(e)=\Lambda(g ; \gamma(e))=\Lambda\left(g \cdot b^{F} ; f(\gamma(e))\right)
$$

Puisque $\gamma(e) \in \Delta_{\mathbb{Q}}$, le dernier terme est dans $N(A)$, ce qui prouve que $\Lambda\left(\mathcal{O}_{T}(g)\right) \subset$ $\left(\mathbb{R}_{+}^{*}\right)_{A}^{\tau_{*}}$. Finalement, le groupe $T$ agit avec orbites discrètes car $\Lambda$ est un homéomorphisme. 


\section{III.4 Le groupe $T$ est un groupe d'isométries}

Ici, on étudie l'action du groupe $T$ dans le système des coordonnées horocycliques. Nous allons montrer que $T$ est un sous-groupe des isométries de $\mathcal{G} r$ pour la métrique horocyclique $d^{b}$ définie à la section II.3. Avant cela, on introduit la notion d'extensions de bracelets.

Soit $\widehat{\mathcal{C}_{n}}$ l'espace des $(n+1)$-bracelets. On définit deux types d'applications sur l'union disjointe $\widehat{\mathcal{C}}=\coprod \widehat{\mathcal{C}_{n}}$, les rotations et les extensions de bracelets.

Soit $n \geq 2$ et $T_{n}$ l'application suivante:

$$
\begin{aligned}
& T_{n}: \hat{\mathcal{C}_{n}} \quad \rightarrow \quad \widehat{\mathcal{C}_{n}} \\
& \left(h_{i}\right)_{i \in \mathbb{Z} /(n+1) \mathbb{Z}} \mapsto\left(h_{i+1}\right)_{i \in \mathbb{Z} /(n+1) \mathbb{Z}}
\end{aligned}
$$

L'application $T_{n}$ définit un homéomorphisme de l'espace $\mathcal{C}_{n}$ d'ordre $n+1$. En particulier, elle définit naturellement une action de $\mathbb{Z} /(n+1) \mathbb{Z}$ sur l'espace $\widehat{\mathcal{C}_{n}}$. C'est notre première application cherchée.

Si maintenant $n \geq 2$ et $i \in \mathbb{Z} /(n+1) \mathbb{Z}$, on définit l'application:

$$
\begin{aligned}
& \begin{array}{llll}
\exp _{i}^{n}: & \widehat{\mathcal{C}_{n}} & \rightarrow & \widehat{\mathcal{C}_{n+1}}
\end{array} \\
& b=\left(h_{i}\right)_{i \in \mathbb{Z} /(n+1) \mathbb{Z}} \mapsto b_{i}^{n}=\left(h_{1} ; \cdots ; h_{i} ; h^{\prime} ; h_{i+1} ; \cdots ; h_{n+1}\right)
\end{aligned}
$$

où $h^{\prime}$ désigne l'unique horocycle de $\mathbb{D}^{2}$ tangent à $h_{i}$ et $h_{i+1}$ et basé dans l'intervalle orienté $] x_{i} ; x_{i+1}$ [ donné par la proposition I.5. La figure III.2 indique une rotation de bracelet suivie d'une extension.

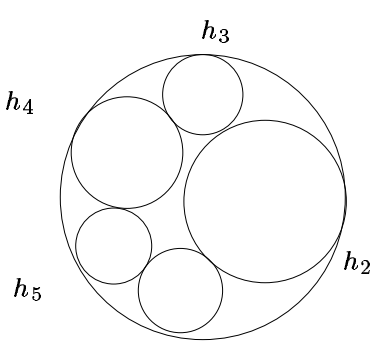

$h_{1}$

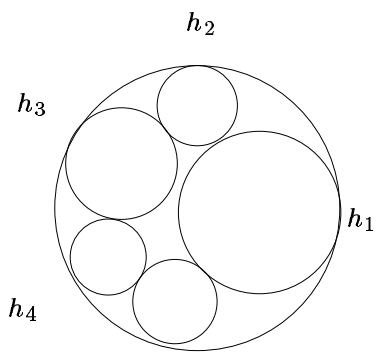

$h_{5}$

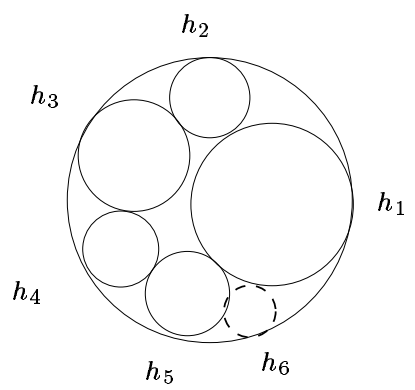

$b$

$T_{n}(b)$

$\exp _{n}^{n}\left(T_{n}(b)\right)$

FIG. III.2 - Une rotation de bracelet suivie d'une extension.

Définition III.4.1. (Extension élémentaire de bracelet) Un bracelet $b^{\prime}$ est appelé extension élémentaire du bracelet $b$ si et seulement si il existe un couple $(i, k)$ tel que $\exp _{i}^{n} \circ T_{n}^{k}(b)=b^{\prime}$ ou bien si $T_{n}^{k}(b)=b^{\prime}$. 
Définition III.4.2. (Extension de bracelet) On dit que le bracelet $b^{\prime}$ est une extension de $b$ si et seulement si il existe une suite finie de bracelets $b=b_{0}, \cdots, b_{k}=b^{\prime}$ telle que pour tout $i, b_{i+1}$ est une extension élémentaire de $b_{i}$.

Pour chaque $n \geq 2$, on possède sur $\widehat{\mathcal{C}_{n}}$ la pseudo-métrique $e_{n}$ définie par:

$$
e_{n}\left(b ; b^{\prime}\right)=S u p_{i \in<1 ; n+1>}\left|l_{i}(b)-l_{i}\left(b^{\prime}\right)\right|
$$

Celle-ci correspond à la métrique $d_{p}^{b} \operatorname{sur} \mathcal{C} r_{p}$ lorsque $n=3 \cdot 2^{p-1}-1$ après quotient par $P S L_{2} \mathbb{R}$. Nous sommes en mesure de montrer la:

Proposition III.4. Le groupe $T$ agit isométriquement sur l'espace $\mathcal{G} r$ muni de la métrique $d^{b}$.

Preuve. - Montrons d'abord que les extensions sont des isométries pour les pseudométriques $\left(e_{n}\right)_{n \geq 2}$. Il suffit de montrer les égalités suivantes pour tout couple $\left(b, b^{\prime}\right)$ d'éléments de $\widehat{\mathcal{C}_{n}}$ :

$$
\begin{gathered}
e_{n}\left(b ; b^{\prime}\right)=e_{n}\left(T_{n}(b) ; T_{n}\left(b^{\prime}\right)\right) \\
e_{n}\left(b ; b^{\prime}\right)=e_{n}\left(\exp _{n}^{i}(b) ; \exp _{n}^{i}\left(b^{\prime}\right)\right) \quad \forall i \in<1 ; n>
\end{gathered}
$$

L'égalité III.3 est évidente puisque $T_{n}$ agit en décalant les horocycles vers la droite. Plus exactement, on a $l_{i}\left(T_{n}(b)\right)=l_{i+1}(b) \quad \forall i \in \mathbb{Z} / n \mathbb{Z}$.

En vertu de la proposition II.2, les coordonnées horocycliques de $\exp _{i}^{n}(b)$ sont: $\left(l_{1}(b), \cdots, l_{i}(b)+1,1, l_{i+1}(b)+1, \cdots, l_{n}(b)\right)$ et on obtient l'égalité III.4. Ainsi, on en déduit que pour toute composition d'extensions élémentaires $a=a_{1} \circ \cdots \circ a_{k}$ partant de $\widehat{\mathcal{C}_{n}}$, et tout couple de bracelets $\left(b, b^{\prime}\right)$ dans $\widehat{\mathcal{C}_{n}}$, on a

$$
e_{p}\left(a(b) ; a\left(b^{\prime}\right)\right)=e_{n}\left(b ; b^{\prime}\right)
$$

où $p$ est un entier tel que $a\left(\widehat{\mathcal{C}_{n}}\right) \subset \widehat{\mathcal{C}_{p}}$.

Considérons maintenant $(f, g) \in \mathcal{G} r_{n}, \gamma \in T$ et $p$ un entier tel que $b k(\gamma) \cup \gamma^{-1}\left(S_{n}\right) \subset$ $S_{p}$. D'après le lemme III.1, $\gamma \cdot b_{p}^{F}$ est une extension de $b_{n}^{F}$ par une application $a$. Puisque $p$ a été choisi pour que $f \circ \gamma$ et $g \circ \gamma$ soient dans $\mathcal{G} r_{p}$, on en déduit que $(f \circ \gamma) \cdot b_{p}^{F}$ et $(g \circ \gamma) \cdot b_{p}^{F}$ sont des bracelets, et qu'ils sont extensions par $a$ des bracelets $f \cdot b_{n}$ et $g \cdot b_{n}$. On en déduit que

$$
e_{3 \cdot 2^{p}-1}\left((f \circ \gamma) \cdot b_{p}^{F} ;(g \circ \gamma) \cdot b_{p}^{F}\right)=e_{3 \cdot 2^{n}-1}\left(f \cdot b_{n}^{F} ; g \cdot b_{n}^{F}\right)
$$

soit encore $d^{b}(f \cdot \gamma ; g \cdot \gamma)=d^{b}(f ; g)$.

Une autre propriété intéressante vérifiée par la métrique $d^{b}$ est la:

Proposition III.5. Le groupe $T$ agit avec orbites discrètes sur l'espace $\mathcal{G} r$ muni de la métrique $d^{b}$.

Preuve. - Nous avons besoin de quelques notations. Si $l \in \mathbb{R}$, on note

$$
Z(l)=\{\delta l+k \mid(\delta, k) \in\{0 ; 1\} \times \mathbb{Z}\} \subset \mathbb{R}
$$


et si $l_{1}, \cdots l_{n}$ sont des réels, on notera

$$
Z\left(l_{1} ; \cdots ; z_{n}\right)=\coprod_{i=1}^{n} Z\left(l_{i}\right)
$$

Remarquons que l'ensemble $Z\left(l_{1} ; \cdots ; l_{n}\right)$ est un sous-ensemble discret de $\mathbb{R}$. Donc, il existe $\epsilon>0$ tel que pour tout couple $\left(l, l^{\prime}\right) \in Z\left(l_{1} ; \cdots ; l_{n}\right)^{2}$, l'inégalité $\left|l-l^{\prime}\right|<\epsilon$ entraine que $l=l^{\prime}$.

Soit $f$ un élément de $\mathcal{G} r_{n}$. Rappelons que l'image de $f$ par l'application $q_{n}: \mathcal{G} r_{n} \rightarrow$ $\left(\mathbb{R}_{+}^{*}\right)^{S_{n}}$ est $q_{n}(f)=\left(l_{x}\left(f \cdot b_{n}^{F}\right)\right)_{x \in S_{n}}$ où $b_{n}^{F}$ est le sous-bracelet de $b^{F}$ basé en $S_{n}$. Notons $Z=Z\left(\left(l_{x}\left(f \cdot b_{n}^{F}\right)\right)_{x \in S_{n}}\right)$.

Supposons que l'orbite de $f$ n'est pas discrète. Alors il existe $\gamma \in T$ tel que $d^{b}(f ; f \cdot \gamma)<\epsilon$. Prenons $p$ suffisamment grand tel que $f$ et $f \cdot \gamma$ sont deux éléments de $\mathcal{G} r_{p}$.

On va montrer que si $x \in S_{p}$, alors les longueurs horocycliques $l_{x}\left(f \cdot b_{p}^{F}\right)$ et $l_{x}((f \cdot$ $\left.\gamma) \cdot b_{p}^{F}\right)$ sont deux éléments de $Z$. Ceci impliquera par définition de $\epsilon$, relativement à l'ensemble $Z$, les égalités $l_{x}\left(f \cdot b_{p}^{F}\right)=l_{x}\left((f \cdot \gamma) \cdot b_{p}^{F}\right)$ pour tout $x \in S_{p}$. Autrement dit, on aura $q_{p}(f)=q_{p}(f \cdot \gamma)$ puis $f=f \cdot \gamma$ car $q_{p}$ est injective.

Soit $m>0$ tel que $\gamma \in \mathcal{G} r_{m}$. Puisque $\gamma$ préserve le bracelet de Ford $b^{F}$, la collection $\gamma \cdot b_{m}^{F}$ est un sous-bracelet du bracelet de Ford. Etant fini, il existe $p \geq 1$ tel que $b_{p}^{F}$ soit une extension de $\gamma \cdot b_{m}^{F}$. Par ailleurs, quitte à prendre $m$ et $p$ plus grand, on peut supposer que $b k(f) \in b k\left(\gamma \cdot b_{m}^{F}\right)$ (rappelons que pour un bracelet $b, b k(b)=$ $\{$ points-base de $b\})$. Ainsi, le bracelet $f\left(b_{p}^{F}\right)$ est une extension du bracelet $f\left(\gamma \cdot b_{m}^{F}\right)=$ $(f \cdot \gamma) \cdot b_{m}^{F}$. Les formules d'extensions élémentaires de bracelets entrainent l'implication suivante pour un bracelet $b=\left(h_{1}, \cdots h_{n}\right)$ :

$$
\text { Si } l_{i}(b) \in Z \quad \forall i \in<1 ; n>\quad \text { alors } \quad l_{i}\left(T_{n}(b)\right) \in Z \text { et } l_{j}\left(\exp _{i}^{n}(b)\right) \in Z
$$

ceci pour tout couple $(i ; j) \in<1 ; n>\times<1 ; n+1>$. Puisque $f\left(b_{p}^{F}\right)$ et $f\left(\gamma \cdot b_{m}^{F}\right)$ sont des extensions du bracelet $f\left(b_{n}^{F}\right)$, on en déduit les égalités $l_{x}\left(f \cdot b_{p}^{F}\right)=l_{x}\left((f \cdot \gamma) \cdot b_{p}^{F}\right)$ pour tout $x \in S_{p}$, ce qui démontre la proposition.

On déduit des deux résultats précédents la propriété suivante, qui s'apparente à une propriété de discontinuité de l'action de $T$ sur $\mathcal{G} r$ dans un sens faible.

Proposition III.6. L'action de $T$ sur $\mathcal{G} r$ possède la propriété suivante:

$$
\forall f \in \mathcal{G} r, \exists V_{f} \text { un voisinage ouvert de } f \mid V_{f} \cdot \gamma \cap V_{f} \neq \emptyset \Rightarrow \gamma \text { fixe } f \text {. }
$$

Preuve. - Supposons que la propriété de l'énoncé ne soit pas réalisée pour un certain $f \in \mathcal{G} r$. La suite d'ensembles $\left(V_{p}=B_{d^{b}}\left(f ; \frac{1}{p}\right)\right)_{p \geq 1}$ est une suite de voisinages ouverts de $f$ pour la topologie faible grâce au lemme II.4. Il existe donc une suite $\left(\gamma_{p}\right)_{p \in \mathbb{N}}$ d'éléments de $T$ telle que $f \cdot \gamma_{p} \neq f$ et $V_{p} \cdot \gamma_{p} \cap V_{p} \neq \emptyset$. Soit $f_{p}$ un élément de cette intersection non vide. En appliquant l'inégalité triangulaire pour la métrique $d^{b}$, on obtient: $d^{b}\left(f ; f \cdot \gamma_{p}\right)<d^{b}\left(f ; f_{p}\right)+d^{b}\left(f_{p} ; f \cdot \gamma_{p}\right)$. Le premier terme est inférieur à $\frac{1}{p}$, 
puisque $f_{p} \in V_{p}$. Le second terme est égal à $d^{b}\left(f_{p} \cdot \gamma_{p}^{-1} ; f\right)$ car $T$ agit par isométries (proposition III.4). Mais $f_{p} \cdot \gamma_{p}^{-1} \in V_{p}$ par définition de $f_{p}$. Ceci entraine l'inégalité $d^{b}\left(f ; f \cdot \gamma_{p}\right)<\frac{2}{p}$ qui contredit la proposition III.5.

\section{III.5 Etude des stabilisateurs}

Pour l'étude des stabilisateurs, nous suivons la démarche de [Gre4]. On va montrer qu'il en existe deux types. Plus exactement, en les points de l'orbite de l'identité, les stabilisateurs sont conjugués à $P S L_{2} \mathbb{Z}$ dans $T$. Par contre, en dehors de cette orbite, les stabilisateurs sont finis et on obtient même une borne supérieure de leur cardinal. Ce résultat nous incite à penser $T$ comme un mapping-class groupe associé à l'espace de "Teichmüller" $\mathcal{G} r$. En effet, dans le cadre des espaces de Teichmüller classique, le mapping-class groupe agit sur l'espace de Teichmüller avec stabilisateurs finis.

Pour étudier les stabilisateurs de l'action de $T$, on procède par une suite de lemmes et propositions.

Lemme III.6. Si $\gamma \in P S L_{2} \mathbb{Z}$ est d'ordre infini et fixe un ensemble fini $A$, alors $A$ possède au plus deux éléments.

Preuve. - Puisque $P S L_{2} \mathbb{Z}$ est un sous-groupe discret de $P S L_{2} \mathbb{R}$, il ne contient pas d'élément elliptique d'ordre infini. Donc, $\gamma$ est soit parabolique, soit hyperbolique. Par conséquent, si $x \in A \backslash F i x(\gamma)$, alors on a $\gamma^{n} x \neq \gamma^{p} x$ pour tout $n \neq p \in \mathbb{Z}$. Ceci est absurde puisque $A$ est fixé et fini. On en déduit que $A$ est inclus dans l'ensemble Fix $(\gamma)$ qui contient 1 ou 2 éléments selon le type d'isométrie de $\gamma$.

Lemme III.7. Si $\gamma \in P S L_{2} \mathbb{Z}$ est d'ordre infini et fixe un élément $f \in \mathcal{G} r$, alors cet élément est l'identité.

Preuve. - Si $f \cdot \gamma=f$, alors il existe $h \in P S L_{2} \mathbb{R}$ tel que $f \circ \gamma=h \circ f$. Puisque $\gamma \in P S L_{2} \mathbb{Z}$, on a $b k(\gamma)=\emptyset$, ce qui fournit la suite d'égalités:

$$
\gamma^{-1} b k(f)=b k(f \circ \gamma)=b k(h \circ f)=b k(f)
$$

car $h \in P S L_{2} \mathbb{R}$. Par conséquent, l'élément $\gamma$ fixe l'ensemble fini $b k(f)$ qui contient donc au plus deux éléments d'après le lemme précédent. On conclut avec la proposition III.7 suivante.

Proposition III.7. Si $f$ est un homéomorphisme de classe CPP, alors il a au moins 4 points de coupure où bien c'est un élément de $P S L_{2} \mathbb{R}$.

Preuve. - Un élément de CPP ne peut avoir qu'un seul point de coupure, puisqu'il serait alors constitué d'un seul morceau. Supposons qu'il ait deux points de coupure. Quitte à composer à gauche et à droite par des éléments de $P S L_{2} \mathbb{R}$, on peut supposer que ses points de coupure sont 0 et $\infty$ et qu'ils sont fixés. Chaque morceau est du type $z \mapsto k z$ et a pour dérivée $k$ en 0 . La condition $\mathcal{C}^{1}$ force le coéfficient $k$ a être le même pour chaque morceau. Ainsi, l'élément $f$ est dans $P S L_{2} \mathbb{R}$. 
Enfin, supposons que cet élément $f$ ait trois points de coupure. On peut également supposer que ses points de coupure sont $0, \infty$ et 1 , et qu'ils sont fixés point par point. Notons $t>0$ la dérivée de $f$ en 0 . Puisque $f$ est l'hyperbolique d'axe $(0 ; \infty)$ sur l'intervalle $[\infty ; 0]$, sa dérivée en $\infty$ est $\frac{1}{t}$. Le même raisonnement sur l'intervalle $[0 ; 1]$ entraine que la dérivée en 1 est égale à $\frac{1}{t}$. Puis, appliqué sur l'intervalle $[1 ; \infty]$, il donne $f^{\prime}(\infty)=t$. Ceci entraine que $t=1$. Donc $f$ est l'identité.

- Remarque: Cette proposition montre l'importance des déformations log-lambda. Ce sont les éléments de $C P P \backslash P S L_{2} \mathbb{R}$ ayant le plus petit nombre de points de coupure.

On s'intéresse à présent à élément quelconque de $T$, toujours d'ordre infini.

Lemme III.8. Si $\gamma \in T$ est d'ordre infini et fixe $f \in \mathcal{G} r$, alors $f$ est dans l'orbite de l'identité.

Preuve. - Nous allons montrer que $D_{x} f \in P S L_{2} \mathbb{Z}$ (le décalage de $f$ en $x$ ) pour tout $x \in \mathbb{S}^{1}$. Ceci impliquera alors l'existence d'un élément $l \in P S L_{2} \mathbb{R}$ tel que $l \circ f \in T$.

La démonstration se décompose en deux cas. Pour les définir, on introduit l'ensemble fini suivant, fixé par $\gamma$ :

$$
A=\bigcap_{n \in \mathbb{Z}} \gamma^{n} b k(f)
$$

Soit $h \in P S L_{2} \mathbb{R}$ tel que $h \circ f=f \circ \gamma$.

- cas 1: l'ensemble $A$ est vide

On déduit du lemme III.2 que $D_{x} f=\left(\gamma_{x}^{r}\right)^{-1} \circ D_{\gamma x} f \circ \gamma_{x}^{l}$. Puis par recurrence, on trouve une suite de couples $\left(a_{n}, b_{n}\right)_{n \in \mathbb{Z}}$ d'éléments de $P S L_{2} \mathbb{Z}$ telle que:

$$
\forall n \in \mathbb{Z} D_{x} f=a_{n} \circ D_{\gamma^{n} x} f \circ b_{n}
$$

Soit $x \in \mathbb{S}^{1}$ et par hypothèse $n \in \mathbb{Z}$ tels que $x \notin \gamma^{-n} b k(f)$. Grâce à l'égalité précédente, on obtient $D_{x} f=a_{n} \circ b_{n} \in P S L_{2} \mathbb{Z}$.

- cas 2: L'ensemble $A$ est non vide

Puisque $f$ conjugue $\gamma$ à $h$, ce dernier fixe l'ensemble fini et non vide $f(A)$. L'élément $\gamma$ est d'ordre infini par hypothèse. Supposons que son conjugué $h$ soit un élément elliptique. Etant d'ordre infini, ses orbites sont denses sur le cercle. Ceci contredit que l'ensemble $f(A)$ est à la fois fini et fixé par $h$. Par conséquent l'élément $h$ est soit parabolique, soit hyperbolique. Cela implique que $\operatorname{card}(A) \leq 2$.

Supposons $\operatorname{card}(A)=2$. Dans ce cas, $h$ est de type hyperbolique et sa dérivée en chacun de ses points fixes est non égale à 1 . Après conjugaison par $f$ qui est de classe $\mathcal{C}^{1}$, la même condition est vérifiée par $\gamma$. Les germes de $\gamma$ de chaque côté de ses points fixes sont donc des éléments hyperboliques de $P S L_{2} \mathbb{Z}$. Or, un élément hyperbolique de $P S L_{2} \mathbb{Z}$ ne peut avoir pour point fixe un rationnel, ce qui contredit que $A \subset \widehat{\mathbb{Q}}$.

On est ramené au cas $\operatorname{card}(A)=1$. Quitte à changer de représentant dans la classe de $f$, on peut supposer que $f$ est l'identité à gauche de $x$ unique élément de $A$. Notons alors $b k(f)=\left(x_{i}\right)_{i=1, n}$ avec $x=x_{1}<x_{2}<\ldots<x_{n}<x_{1}$ et $f=f_{i}$ à gauche de $x_{i}$ de 
sorte que $f_{1}=i d$. Puisque $x_{2} \notin A$, il existe $n_{2}$ tel que $\gamma^{-n_{2}} \cdot x_{2}$ ne soit pas un point de coupure de $f$. Par le même argument sur le décalage, on en déduit qu'il existe deux éléments $a_{n_{2}}$ et $b_{n_{2}}$ de $P S L_{2} \mathbb{Z}$ tels que $D_{x_{2}} f=a_{n_{2}} \circ b_{n_{2}} \in P S L_{2} \mathbb{Z}$.

L'argument se répète alors en chaque $x_{i}$ puisque $x_{i} \notin A$. On obtient ainsi que $f_{i} \in P S L_{2} \mathbb{Z}$ pour tout $i \in<1, n>$ et ce nouveau représentant est un élément du groupe $T$.

On peut résumer la situation dans le théorème suivant:

Théorème III.1. Soit $f \in \mathcal{G} r$ et $\gamma$ un élément de $T$ fixant $f$. Soit $\gamma$ est d'ordre fini, soit il est conjugué dans $T$ à un élément de $P S L_{2} \mathbb{Z}$. Dans ce dernier cas, $f$ est dans l'orbite de l'identité.

Preuve. - Supposons que $\gamma$ est d'ordre infini. D'après le lemme III.8, il existe $l \in P S L_{2} \mathbb{R}$ tel que $g=l \circ f \in T$. Ayant de plus $h \circ f=f \circ \gamma$, où $h \in P S L_{2} \mathbb{R}$, on en déduit que $g \gamma g^{-1}=l h l^{-1} \in P S L_{2} \mathbb{R} \cap T=P S L_{2} \mathbb{Z}$ et donc $g$ conjugue $\gamma$ dans $T$ à un élément de $P S L_{2} \mathbb{Z}$. Le lemme III.8 montre que $f$ est dans l'orbite de l'identité.

- Remarque: Dans la démonstration du lemme III.8, nous avons introduit l'ensemble $A$ afin d'éviter d'avoir recours à un résultat de Ghys et Sergiescu (utilisé dans [Gre4]) concernant le nombre de rotations d'un élément du groupe de Thompson. Dans [Ghy-Ser], les auteurs montrent que le nombre de rotation d'un élément de $T$ est toujours rationnel. Ce résultat est en fait le corollaire d'un théorème de lissage du groupe $T$, couplé à un théorème de Denjoy. Nous avons préféré ici ne pas avoir recours à ce résultat.

Grâce au théorème III.1, nous savons qu'un élément de $T$ fixant un point en dehors de l'orbite de l'identité, est nécessairement d'ordre fini. On a mieux grâce au résultat suivant.

Théorème III.2. Si $f$ n'est pas dans l'orbite de l'identité, alors le stabilisateur de $f$ est un groupe cyclique fini, d'ordre inférieur ou égal à $b k(f)$.

Preuve. - Montrons d'abord que les éléments de stab $(f)$ ont un ordre borné par $b k(f)$. On en déduira le théorème par un argument de densité sur le cercle.

Soit $\gamma \in \operatorname{stab}(f)$. D'après le théorème III.1, il est d'ordre fini égal au cardinal des orbites du groupe cyclique $\langle\gamma\rangle$ sur $\mathbb{S}^{1}$. Il suffit donc d'exhiber un élément $x$ de $b k(f)$ dont l'orbite par $\langle\gamma\rangle$ est contenue dans $b k(f)$. Supposons qu'il n'en existe pas. Alors, pour tout $x$ dans $b k(f)$, il existe $k_{x}$ dans $\mathbb{Z}$ tel que $\gamma^{k_{x}}(x) \notin b k(f)$. Ce qui entraine en particulier les égalités:

$$
D_{f(x)} \gamma^{k} f^{-1}=D_{f(x)} f \gamma^{k} f^{-1}=i d
$$

(la deuxième égalité traduit simplement que le conjugué de $\gamma^{k}$ par $f$ est dans $P S L_{2} \mathbb{R}$ ). Le lemme III.2 donne l'égalité:

$$
D_{f(x)} \gamma^{k} f^{-1}=\left(\left(f^{-1}\right)_{f(x)}^{r}\right)^{-1} \circ D_{x} \gamma^{k} \circ\left(f^{-1}\right)_{f(x)}^{l}
$$

L'égalité III.5 entraine alors que $D_{x} f=\left[D_{x} \gamma^{k}\right]^{-1}$ est un élément de $P S L_{2} \mathbb{Z}$ pour tout $x \in b k(f)$. Ce qui contredit l'hypothèse que $f$ n'est pas dans l'orbite de l'identité. 
Montrons maintenant que stab $(f)$ est conjugué à un sous-groupe de $\mathbb{S}^{1}$. Soit $\gamma, \gamma^{\prime}$ deux éléments de stab $(f)$ et $h, h^{\prime}$ les deux isométries correspondantes. Ce sont les conjugués de $\gamma$ et $\gamma^{\prime}$ par $f$. Par conséquent, le commutateur de $h$ et $h^{\prime}$ est le conjugué par $f$ du commutateur de $\gamma$ et $\gamma^{\prime}$. En particulier $\left[\gamma, \gamma^{\prime}\right]$ stabilise aussi $f$ et donc est d'ordre fini. Il en est de mème pour $\left[h, h^{\prime}\right]$.

On affirme que $\left[h, h^{\prime}\right]$ est l'identité. En effet, puisque $h$ est d'ordre fini, il est elliptique. Le théorème 7.39.2 dans [Bea] montre que le commutateur d'un élément elliptique avec un élément quelconque de $P S L_{2} \mathbb{R}$ est soit hyperbolique, soit l'identité. On en déduit que $f$ conjugue stab $(f)$ à un sous-groupe de $\mathbb{S}^{1}$, vu comme $S O_{2}(\mathbb{R}) /\left\{ \pm I_{2}\right\}$.

Les sous-groupes de $\mathbb{S}^{1}$ sont denses où finis. Or, dans le cas de $s t a b(f)$, il n'y a pas d'éléments proches de l'identité. Plus exactement, l' arc ouvert délimité par les angles 0 et $\frac{2 \pi}{\operatorname{card}(b k(f))}$ n'intersecte pas $s t a b(f)$, puisque les éléments de $s t a b(f)$ sont d'ordre au plus $b k(f)$. On en déduit que $s t a b(f)$ est un sous-groupe fini de $\mathbb{S}^{1}$. Il est cyclique fini et d'ordre plus petit ou égal à $\operatorname{card}(b k(f))$.

\section{III.6 Analogie avec les espaces de Teichmüller}

Comme nous l'avons signalé précédemment, l'étude de $T$ sur l'espace contractile $\mathcal{G} r$ fait penser à celle du mapping-class group sur l'espace de Teichmüller d'une surface de Riemann. Cependant, dans le cas présent, nous avons vu que les points de l'orbite de l'identité de $\mathcal{G} r$ ont leurs stabilisateurs conjugués au groupe modulaire $P S L_{2} \mathbb{Z}$. Une idée pour éviter ce problème consiste à étudier l'espace $\mathcal{G} r^{\sim}$ que l'on définit comme l'espace $\mathcal{G} r \backslash \mathcal{O}_{T}(i d)$. Dans [Gre4], l'auteur montre que l'espace $\mathcal{G} r \sim$ est faiblement contractile. A l'aide des coordonnées log-lambda, nous avons un résultat plus précis:

Proposition III.8. L'espace $\mathcal{G} r^{\sim}$ est faiblement contractile pour la topologie faible.

Preuve. - Puisque l'orbite de l'identité est discrète, il suffit de montrer que l'espace $\mathcal{G} r^{*}=\mathcal{G} r \backslash\{i d\}$ est faiblement contractile. Le théorème II.2, montre, modulo le changement de paramêtre $x \mapsto \log \left(\frac{x}{\sqrt{2}}\right)$ sur chaque coordonnée, que $\mathcal{G} r^{*}$ est homéomorphe à l'espace $\mathbb{R}^{(\infty)} \backslash\{0\}$, introduit au pargraphe I.1.e. Cet espace est contractile d'après la proposition I.2.

Cette proposition et le théorème III.1 fournissent le corollaire suivant.

Corollaire III.1. L'espace $\mathcal{G} r / F^{\prime}$ est un espace $K\left(F^{\prime}, 1\right)$. Si $H$ est un sous-groupe de $T$ sans torsion, alors $\mathcal{G} r^{\sim} / H$ est un espace $K(H, 1)$.

Preuve. - Considérons la première assertion. Tout d'abord, le groupe $F^{\prime}$ ne possède pas d'éléments d'ordre fini. En effet, puisque les éléments de $F^{\prime}$ sont égaux à l'identité au voisinage de $\infty$, le groupe $F^{\prime}$ se plonge dans le groupe des homéomorphismes croissants de $\mathbb{R}$. Celui-ci est sans torsion. Il est à présent facile de montrer que $F^{\prime}$ agit librement sur l'espace $\mathcal{G} r$. D'aprés le théorème III.1, et ce qui précède, si $\gamma \in F^{\prime}$ fixe un élément de $\mathcal{G} r$, il est nécessairement conjugué dans $T$ à un élément de $P S L_{2} \mathbb{Z}$. 
Mais alors cet élément a son germe en $g(\infty)$, où $g$ est l'élément de $T$ conjugant $\gamma$ dans $T$, égal à l'identité. Etant dans $P S L_{2} \mathbb{R}$, cet élément est l'identité et $\gamma$ aussi. De plus, $F^{\prime}$ agissant librement, la proposition III.6 montre que cette action est proprement discontinue. Puisque $\mathcal{G} r$ est contractile (corollaire II.1), l'espace quotient $\mathcal{G} r / F^{\prime}$ est effectivement un espace $K\left(F^{\prime} ; 1\right)$.

Pour la deuxième assertion, toujours d'après le théorème III.1 et par définition de $\mathcal{G} r^{\sim}$, si $\gamma \in H$ fixe un élément de $\mathcal{G} r^{\sim}$, il est d'ordre fini, mais ceci est impossible car on a supposé $H$ sans torsion. La proposition III.8 permet de conclure la deuxième assertion.

L'analogie entre l'action du mapping-class group sur l'espace de Teichmüller et celle de $T$ sur $\mathcal{G} r$ entraine une question intéressante sur la métrique $d^{b}$. En effet, dans le cas classique, l'espace de Teichmuller $\mathcal{T}_{g}^{s}$ d'une surface $F=F_{g}^{s}$ est muni de la métrique de Teichmüller, notée $d_{\mathcal{H}}$, et celle-ci est invariante par l'action du mapping-class group $M C_{g}^{s}$. Dans [Roy], l'auteur montre le:

Théorème III.3. (Royden [Roy]) Le groupe des isométries de $\mathcal{T}_{g}^{s}$ est le mapping-class group. $M C_{g}^{s}$.

Ainsi, les isométries de l'espace de Teichmüller sont réalisées par l'action du groupe

Dans le cadre universel, il existe un résultat analogue que nous rappelons ici. L'espace de Teichmüller de Bers T(1) est muni d'une structure presque-complexe, c'est à dire d'une famille lisse d'opérateurs $J=\left(J_{x}\right)_{x \in T(1)}$ sur les espaces tangents $\left(T_{x} T(1)\right)_{x \in T(1)}$ telle que $J_{x}^{2}=-I d$. Un difféomorpisme de $T(1)$ est un automorphisme (presque-complexe) si et seulement si sa dérivée commute avec $J$. Notons que pour $f \in Q S$ (le groupe des homéomorphismes quasi-symétriques du cercle) l'application:

$$
\rho_{f}:[h] \in T(1) \mapsto[h \circ f] \in T(1)
$$

est un automorphisme de $T(1)$. Autrement dit, on a un plongement du groupe $Q S$ dans $A u t_{J}(T(1))$.

Théorème III.4. (Gardiner, Harvey [Gar-Har]) Le groupe QS coïncide exactement avec Aut A $_{\text {(T) }}(1)$.

Revenons dans notre cadre du groupe de Thompson. Une question se pose alors.

- Question: Le groupe des isométries de $\mathcal{G} r$ muni de $d^{b}$ est-il égal au groupe $T$ ?

\section{III.7 Un classifiant explicite pour $T$.}

Le corollaire III.1 fournit un classifiant explicite du groupe $F^{\prime}$, en contexte $C P P$. Par contre, $\mathcal{G} r / T$ n'est pas un espace classifiant pour le groupe $T$. Il faut supprimer les stabilisateurs de l'action. Ce qui nous incite à se placer sur $\widehat{\mathcal{G r}}$ sur lequel l'action de $T$ 
est libre. Puisque $\widehat{\mathcal{G r}}$ a le type d'homotopie du cercle ( $\mathcal{G} r$ est contractile), on le déplie dans l'espace des difféomorphismes de $\mathbb{R}$.

Considérons le revêtement universel du cercle $p: t \in \mathbb{R} \mapsto[t] \in \mathbb{R} / \mathbb{Z}$.

Si $\tilde{f}$ est un homéomorphisme de $\mathbb{R}$, vérifiant l'égalité

$$
\tilde{f}(x+1)=\tilde{f}(x)+1 \quad \forall x \in \mathbb{R}
$$

alors il se projète en un homéomorphisme du cercle $f$ du cercle définie par $f([t])=$ $[\tilde{f}(t)]$.

Définition III.7.1. Soit

$$
\widetilde{\text { оотео }^{+}}\left(\mathbb{S}^{1}\right)=\{\tilde{f}: \mathbb{R} \rightarrow \mathbb{R} \mid \tilde{f}(x+1)=\tilde{f}(x)+1 \quad \forall x \in \mathbb{R}\}
$$

le revêtement universel de $\mathrm{Homeo}^{+}\left(\mathbb{S}^{1}\right)$ (celui-ci a le type d'homotopie du cercle). Si $A$ est un sous-ensemble de $\operatorname{Homeo}^{+}\left(\mathbb{S}^{1}\right)$, on note $\tilde{A}$ son relevé dans homeo+ $\left(\mathbb{S}^{1}\right)$. De cette façon, on définit l'espace $\widetilde{\widehat{\mathcal{G r}}}$ et le groupe $\widetilde{T}$.

Cela donne la suite exacte courte de groupes $0 \rightarrow \mathbb{Z} \rightarrow \widetilde{T} \rightarrow T \rightarrow 0$. Celle-ci est utilisée pour détecter un classifiant du groupe $T$. Pour trouver un tel classifiant, on commence par le:

Lemme III.9. L'espace $\widetilde{\widehat{\mathcal{G} r}}$ est contractile. De plus, le groupe $\widetilde{T}$ agit sur $\widetilde{\widehat{\mathcal{G r}}}$ et donne au quotient un espace homéomorphe à l'espace $\widehat{\mathcal{G} r} / T$.

Preuve. - Tout d'abord la fibration

$$
P S L_{2} \mathbb{R} \rightarrow \widehat{\mathcal{G r}} \rightarrow \mathcal{G} r
$$

indique que $\widehat{\mathcal{G r}}$ est homéomorphe au produit $\mathcal{G} r \times P S L_{2} \mathbb{R}$, puisque $\mathcal{G} r$ est contractile (corollaire II.1). Ainsi, le revêtement universel de $\widehat{\mathcal{G} r}$ est homéomorphe au produit $\mathcal{G} r \times \widetilde{P S L_{2} \mathbb{R}}$ qui est contractile.

Pour la deuxième assertion du lemme, l'application composée

$$
\widetilde{\widehat{\mathcal{G}} r} \rightarrow \widehat{\mathcal{G} r} \rightarrow \widehat{\mathcal{G} r} / T
$$

est continue et surjective et passe au quotient en une application continue

$$
\phi: \tilde{f}[\widetilde{T}] \in \widetilde{\widehat{\mathcal{G}} r} / \widetilde{T} \mapsto f[T] \in \widehat{\mathcal{G} r} / T
$$

avec les notations évidentes sur les classes d'équivalence.

Pour l'injectivité, supposons que $\phi(\tilde{f}[\widetilde{T}])=\phi(\tilde{g}[\widetilde{T}])$. Il existe $\gamma \in T$ tel que $g=f \circ \gamma$. Soit $\tilde{\gamma}$ un relevé de $\gamma$ dans $\widetilde{T}$ et $k \in \mathbb{Z}$ tel que $\tilde{g}=\widetilde{f \circ \gamma}+k$. Ayant $\widetilde{f \circ \gamma}+k=\tilde{f} \circ(\tilde{\gamma}+k)$, on en déduit que $\tilde{f}[\widetilde{T}]=\tilde{g}[\widetilde{T}]$, car $\tilde{\gamma}+k \in \widetilde{T}$. Ce qui finit la démonstration du lemme. 
La fibration en cercle

$$
P S O_{2}(\mathbb{R})=\mathbb{S}^{1} \rightarrow \widehat{\mathcal{G} r} / T \times E \mathbb{S}^{1} \rightarrow \widehat{\mathcal{G} r} / T \times \mathbb{S}^{1} E \mathbb{S}^{1}
$$

donne alors le corollaire suivant.

Corollaire III.2. L'espace $\widehat{\mathcal{G r}} / T$ est un classifiant du groupe $\widetilde{T}$. L'espace $\widehat{\mathcal{G r}} / T \times_{\mathbb{S}^{1}} E \mathbb{S}^{1}$ est un classifiant du groupe $T$.

Preuve. - Puisque $\widetilde{T}$ agit proprement discontinument et librement sur l'espace contractile $\widetilde{\widehat{\mathcal{G}} r}$, on déduit du lemme III.9 que $\widehat{\mathcal{G} r} / T$ est un classifiant de $\tilde{T}$. La fibration III.6 donne la suite exacte longue d'homotopie

$$
\begin{aligned}
& \rightarrow \quad 0 \quad \rightarrow \quad \pi_{i}\left(\widehat{\mathcal{G} r} / T \times_{\mathbb{S}^{1}} E \mathbb{S}^{1}\right) \rightarrow 0 \\
& \rightarrow \quad \ldots \quad \rightarrow \pi_{2}\left(\widehat{\mathcal{G} r} / T \times_{\mathbb{S}^{1}} E \mathbb{S}^{1}\right) \rightarrow \pi_{1}\left(\mathbb{S}^{1}\right) \\
& \rightarrow \pi_{1}\left(\widehat{\mathcal{G} r} / T \times E \mathbb{S}^{1}\right) \rightarrow \pi_{1}\left(\widehat{\mathcal{G} r} / T \times_{\mathbb{S}^{1}} E \mathbb{S}^{1}\right) \rightarrow 0
\end{aligned}
$$

On en déduit que $\pi_{i}\left(\widehat{\mathcal{G} r} / T \times_{\mathbb{S}^{1}} E \mathbb{S}^{1}\right)=0 \forall i \geq 2$. Par ailleurs d'après ce qui précède $\pi_{1}\left(\widehat{\mathcal{G} r} / T \times E \mathbb{S}^{1}\right)=\widetilde{T}$. On en déduit la suite exacte courte

$$
0 \rightarrow \pi_{1}\left(\mathbb{S}^{1}\right) \rightarrow \widetilde{T} \rightarrow \pi_{1}\left(\widehat{\mathcal{G} r} / T \times_{\mathbb{S}^{1}} E \mathbb{S}^{1}\right) \rightarrow 0
$$

qui couplée avec la suite exacte

$$
0 \rightarrow \mathbb{Z} \rightarrow \widetilde{T} \rightarrow T \rightarrow 0
$$

donne l'égalité $\pi_{1}\left(\widehat{\mathcal{G} r} / T \times_{\mathbb{S}^{1}} E \mathbb{S}^{1}\right)=T$. En effet, le générateur du $\pi_{1}$ de la fibre correspond au lacet $\mathrm{PSO}_{2}(\mathbb{R}) \subset \widehat{\mathcal{G} r}$. Celui-ci se relève dans $\widehat{\widehat{\mathcal{G} r}}$ en un chemin $\rho:[0 ; 1] \rightarrow$ $\widetilde{\widehat{\mathcal{G r}}}$ vérifiant $\rho(s, x)=x+s$ pour tout $(s, x) \in[0 ; 1] \times \mathbb{R}$. Ainsi, on a $\rho(1, x)=\rho(0, x) \circ c$ où $c: x \in \mathbb{R} \mapsto x+1 \in \mathbb{R}$ est le générateur du centre de $\widetilde{T}$, ce qui montre que le $\pi_{1}$ de la fibre est exactement le centre de $\widetilde{T}$ et conclut la démonstration. 


\section{Chapitre IV}

\section{Homologie du groupe de Thompson et espaces de lacets}

Ce dernier chapitre donne une application de la géométrie CPP, portant sur l'homologie des groupes de Thompson $F^{\prime}, T$ et $\tilde{T}$. Homologiquement, les classifiants de ces groupes sont des espaces de lacets de la sphère $\mathbb{S}^{3}$. Nous tentons de développer une version géométrique du théorème suivant dans le contexte CPP.

Théorème IV.1. (Ghys-Sergiescu, [Ghy-Ser]) Il existe des applications continues

$$
\begin{aligned}
& B F^{\prime} \stackrel{a}{\longrightarrow} \Omega \mathbb{S}^{3} \\
& B \widetilde{T} \stackrel{b}{\longrightarrow} \Lambda \mathbb{S}^{3} \\
& B T \stackrel{c}{\longrightarrow} \mathcal{L} \mathbb{S}^{3}
\end{aligned}
$$

qui induisent des isomorphismes en homologie entière.

La démonstration initiale de ce théorème se situe dans le contexte PL et utilise des méthodes de topologie algébrique provenant de la théorie des feuilletages. Bien que de nature géométrique, les applications $a b$ et $c$ sont difficiles à décrire. Nous effectuerons un survol géométrique succint de la démonstration originale de ce théorème dans la section IV.1.

Nous construisons ensuite des nouveaux modèles (géométriquement transparents) des applications continues $a b$ et $c$, à partir de la géométrie CPP. L'idée initiale de la construction se trouve dans [Gre4] et utilise le modèle de James $J \mathbb{S}^{1}$ de l'espace des lacets pointés $\Omega \mathbb{S}^{2}$. L'auteur construit une application $\varphi$ de $B F^{\prime}$ à valeurs dans $J \mathbb{S}^{1}$ qui se relève (via la fibration de Hopf) en une application $\tilde{\varphi}$ dans $\Omega \mathbb{S}^{3}$ qui est un isomorphisme en homologie. On expliquera sa démarche dans la section IV.2.

Pour adapter la construction de $\varphi$ aux applications $b$ et $c$, nous développons, en section IV.4, un nouveau modèle (intéressant en soi) de $\Lambda \mathbb{S}^{3}$, noté $M \mathbb{S}^{2}$ (théorème IV.7 et corollaire IV.2) en "relevant" le modèle de Cohen de $\Lambda \mathbb{S}^{2}$ (section IV.3 et [Coh]). Ce dernier possède deux versions définies respectivement à l'aide des espaces de configurations ordonnées de points du cercle (pour la version notée $\left(L \mathbb{S}^{1}\right)_{\bullet}$ ), et d'intervalles 
disjoints deux à deux du cercle (pour la version notée $L \mathbb{S}^{1}$ ). Nous définissons donc deux espaces combinatoires, $M \mathbb{S}^{2}$ et $\left(M \mathbb{S}^{2}\right)$., faiblement homotopiquement équivalents. Le corollaire IV.2 fournit une équivalence faible d'homotopie entre $M \mathbb{S}^{2}$ (le modèle des intervalles) et $\Lambda \mathbb{S}^{3}$.

Dans la section IV.5, nous donnons une application $\psi$ de $\widehat{\mathcal{G r}} / T$ (classifiant de $\widetilde{T})$ à valeurs dans le modèle $\left(M \mathbb{S}^{2}\right)$. (corollaire IV.3). Notre construction de $\psi$ n'est pas complètement explicite dans la mesure où elle fait intervenir la primitive $\theta$ d'un cocycle nul $[\mu] \in H^{1}\left(T ; \mathcal{C}^{0}\left(\widehat{\mathcal{G} r} ; \mathbb{S}^{1}\right)\right)$, que nous ne savons pas calculer. La nullité de $[\mu]$ est expliquée au corollaire IV.5 de la section IV.6.

Pour calculer l'homologie de $\psi$, on utilise le fait que celle-ci est $\mathbb{S}^{1}$-équivariante pour les actions du cercle, par composition à gauche de $P S O_{2}(\mathbb{R})$ sur $\widehat{\mathcal{G r}}$ et par rotation à la source sur $\Lambda \mathbb{S}^{3}$. L'équivariance utilise la condition ( $\mathbb{S}^{1}$-INV) de l'introduction portant $\operatorname{sur} \theta$. Les calculs entrepris pour donner une formule de $\theta$ et la géométrie CPP (sousjacente à $\psi$ ) indiquent la pertinence de cette condition que nous supposerons vraie, bien que non démontrée.

Puis, en utilisant l'homologie de $\widetilde{T}$ [Ghy-Ser], on montre dans la section IV.6 que $\psi$ donne un isomorphisme en homologie (théorème IV.9). On a ainsi construit une version quasiment explicite de l'application $b$ du théorème IV.1. Le procédé de construction de Borel (section IV.5.d) appliqué à $\psi: B \widetilde{T} \rightarrow \Lambda \mathbb{S}^{3}$ ( $\psi$ est $\mathbb{S}^{1}$-équivariante sous l'hypothèse précédente) donne $\psi \times_{\mathbb{S}^{1}} i d: B T \rightarrow \mathcal{L S}^{3}$. Celle-ci fournit une interprétation de $c$ (corollaire IV.7).

Le dernier paragraphe est consacré au cas de $F^{\prime}$. Une autre démonstration est donnée, ne dépendant que de la connaissance de la structure additive de l'homologie de $F$ ([Bro-Geo], [Ste]), et faisant appel à des techniques plus élémentaires que celles utilisées dans [Ghy-Ser].

\section{IV.1 Le théorème de Ghys et Sergiescu}

\section{IV.1.a Les espaces de lacets}

Afin d'interpreter le théorème IV.1, nous utilisons des modèles combinatoires des espaces de lacets. Rappelons la définition des différents types d'espaces de lacets.

On munit $\mathbb{S}^{1}=\mathbb{R} / \mathbb{Z}$ du point-base 0 qui correspond au nombre rationnel $\frac{0}{1}$ sur la droite projective réelle, via la fonction tangente:

$$
\tan (\pi \cdot): x \in \mathbb{R} / \mathbb{Z} \mapsto \tan (\pi x) \in \mathbb{R} \cup \infty
$$

Rappelons que $E \mathbb{S}^{1}=\lim _{n \rightarrow \infty} \mathbb{S}^{2 m+1}$ est l'espace total du $\mathbb{S}^{1}$-fibré universel (défini à homotopie près), de base $B \mathbb{S}^{1}=\lim _{m \rightarrow \infty} \mathbb{C P}^{m}$. Ce fibré est limite directe de la suite des $\mathbb{S}^{1}$-fibrés de Hopf

$$
\mathbb{S}^{1} \rightarrow \mathbb{S}^{2 m+1} \rightarrow \mathbb{C P}^{m}
$$


sous les inclusions $\mathbb{S}^{2 m+1} \subset \mathbb{S}^{2 m+3}$. L'action du cercle sur $E \mathbb{S}^{1}$ se fait par multiplication scalaire en restriction aux sphères.

Définition IV.1.1. (Espaces de lacets)

Soit $(X, *)$ un espace topologique pointé. On définit les espaces suivants:

$$
\begin{gathered}
\Omega X=\left\{f: \mathbb{S}^{1} \rightarrow X \mid \mathrm{f} \text { est continue et } f(0)=*\right\} \\
\Lambda X=\left\{f: \mathbb{S}^{1} \rightarrow X \mid \text { f est continue }\right\}
\end{gathered}
$$

Le cercle agit par rotation à la source sur $\Lambda \mathbb{S}^{1}$. On note

$$
\mathcal{L} X=\Lambda X \times_{\mathbb{S}^{1}} E \mathbb{S}^{1}
$$

le quotient de $\Lambda X \times \mathbb{S}^{1}$ par l'action diagonale de $\mathbb{S}^{1}$. Ces trois espaces sont appelés respectivement: espace des lacets pointés sur $X$, espace des lacets libres sur $X$, et espace des lacets libres non paramétrés sur $X$.

On munit ces espaces de la topologie de la convergence uniforme pour $\Omega X$ et $\Lambda X$, et de la topologie quotient sur $\mathcal{L} X$.

\section{IV.1.b Un survol géométrique de la démonstration de Ghys et Sergiescu}

Afin de mieux comprendre le théorème IV.1, enoncé et démontré par des méthodes quantitatives, nous allons en donner une explication géométrique, en terme de feuilletages. En effet, nous envisageons les groupes de Thompson $F^{\prime}, T$ et $\tilde{T}$ comme groupes discrets de difféomorphismes du cercle.

Or, ceux-ci apparaissent naturellement dans l'étude des feuilletages de codimension 1 avec structure transverse sur les variétés différentiables. Nous rappellerons notamment un feuilletage de Reeb sur la sphere $\mathbb{S}^{3}$ transversalement linéaire par morceaux. La démonstration de l'équivalence d'homologie $a$ se décompose en deux étapes:

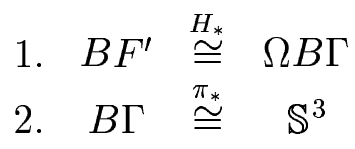

où $\Gamma$ est un pseudo-groupe à définir, et $\stackrel{H_{*}}{\cong}\left(\right.$ resp. $\left.\stackrel{\pi_{*}}{\cong}\right)$ signifie équivalence en homologie (resp. équivalence d'homotopie). Pour chacune, nous rappelons le résultat algébrique quantitatif, puis une interpretation géométrique qualitative.

Faisons au préalable, un bref rappel sur les notions de feuilletage muni d'une structure transverse et de pseudo-groupe. On se place dans le cadre de la codimension 1.

Une famille $\Gamma$ d'homéomorphismes locaux du cercle (ou de la droite réelle) est un pseudo-groupe si elle contient l'identité et est stable pour les opérations suivantes:

1. restriction à un ouvert de la source 


\section{2. composition}

3. passage à l'inverse

4. réunion

L'exemple type de pseudo-groupe dans notre situation est le pseudo-groupe $\Gamma\left(\mathbb{S}^{1}\right)$ des homéomorphismes locaux de $\mathbb{S}^{1}$ qui sont de la forme $x \mapsto 2^{n} x+\frac{p}{2^{q}}$ par morceaux, à points de coupure dyadiques. Un autre exemple, plus connu, est celui de tous les difféomorphismes locaux de $\mathbb{R}$ de classe $\mathcal{C}^{\infty}$. On le note $\Gamma^{\infty}$. Rappelons enfin qu'il existe une notion d'espace classifiant associé à un pseudo-groupe. Par définition, $B \Gamma$ est le classifiant du groupoïde $\pi_{\Gamma}$ des gèrmes associés à $\Gamma$.

Un feuilletage de codimension 1 , muni d'une $\Gamma$-structure sur une variété différentiable $M^{n}$ est une partition localement de la forme $\mathbb{R}^{n-1} \times \mathbb{R}$ de $M^{n}$ en sous-variétés de codimension 1, connexes, appelées feuilles et dont les applications de transitions sont de type $\Gamma$. Nous renvoyons à [God] pour un panorama très large sur l'étude des feuilletages.

\section{Première étape}

Dans [Ghy-Ser], les auteurs s'inspirent d'un théorème fondamental dû à Mather [Mat], qui établit une équivalence d'homologie entre le classifiant du groupe discret Dif $f_{c}^{\infty}(\mathbb{R})$ des difféomorphismes $\mathcal{C}^{\infty}$ à support compact et l'espace des lacets sur le classifiant du pseudo-groupe $\Gamma^{\infty}$. Une version analogue de ce résultat existe pour les groupes $F^{\prime}$ et $\widetilde{T}$.

Théorème IV.2. (Ghys-Sergiescu [Ghy-Ser] 1987) Il existe des équivalences d'homologie entre l'espace $B F^{\prime}$ (resp. $\left.B \widetilde{T}\right)$ et $\Omega B \Gamma\left(\mathbb{S}^{1}\right)$ (resp. $\Lambda B \Gamma\left(\mathbb{S}^{1}\right)$ ).

Nous décrivons l'esquisse de la construction d'une telle équivalence pour le groupe $F^{\prime}$. Notons que $F^{\prime}$ s'identifie à un sous-groupe discret de Homeo $_{c}^{+}([0 ; 1[$ ) (l'indice c signifie à support compact). Le groupe $\mathbb{Z}^{2}$ se plonge dans $F^{\prime}$ par l'application qui à $(1,0)$ (resp. $(1,0))$ associe l'élément $u$ (resp. $v$ ) de $F^{\prime}$ explicité au paragraphe IV.6.b. On a ainsi une représentation $h: \Pi_{1}(\mathbb{T}) \rightarrow$ Homeo $_{c}^{+}(] 0 ; 1[)$. La suspension de $h$ est alors l'espace quotient $E_{h}=\mathbb{R}^{2} \times[0,1] / \mathbb{Z}^{2}$ où $\mathbb{Z}^{2}$ agit à gauche par translations et à droite par la représentation $h . E_{h}$ possède le feuilletage horizontal $\mathcal{F}=\left(\mathbb{R}^{2} \times\{x\}\right)_{x \in[0,1]} / \mathbb{Z}^{2}$ trivial en dehors d'un compact. Il passe ainsi au quotient en un feuilletage sur la suspension du tore $\Sigma \mathbb{T}^{2}$. Ce feuilletage singulier est muni d'une $\Gamma$-structure puisque la suspension $h$ est à valeur dans $P L_{2}\left(\mathbb{S}^{1}\right)$. Il est donc classifié par un élément de $\left[\Sigma \mathbb{T}^{2}, B \Gamma\right]=\left[B \mathbb{Z}^{2}, \Omega B \Gamma\right]$ $([X, Y]$ désignant l'ensemble des classes d'homotopie d'applications continues de $X$ vers $Y)$. Une construction analogue donnerait un élément de $\left[B F^{\prime}, \Omega B \Gamma\right]$ fournissant l'équivalence d'homologie recherchée.

\section{Deuxième étape}

Il s'agit d'interpreter le résultat suivant du à Greenberg. 
Théorème IV.3. (Greenberg [Gre1] 1987) Le classifiant de $\Gamma$ a le type d'homotopie de la sphère $\mathbb{S}^{3}$.

La démonstration originale de ce résultat est difficile et fait appel à une étude poussée des pseudo-groupes. Cependant, nous allons en donner une interprétation géométrique à l'aide de l'holonomie d'un feuilletage de Reeb sur la sphère $\mathbb{S}^{3}$ en s'inspirant de [Tsu].

Soit $f_{+}$(resp. $f_{-}$) l'homothétie de rapport 2 (resp. $\frac{1}{2}$ ) de $\mathbb{R}^{3}$. Le feuilletage horizontal $\mathcal{F}_{h}$ de $\mathbb{R}^{3}$ est laissé invariant par $f_{+}$et $f_{-}$. Sa restriction à $\mathbb{R}_{+}^{3} \backslash(0,0,0)$ se projète en un feuilletage de Reeb $\mathcal{F}_{+}\left(\right.$resp. $\left.\mathcal{F}_{-}\right)$du tore solide $\mathbb{R}_{+}^{3} \backslash(0,0,0) /<f_{+}>$(resp. $\left.\mathbb{R}_{+}^{3} \backslash(0,0,0) /<f_{-}>\right)$. Ces deux tores solides se recollent en leur bord pour donner un feuilletage de codimension 1 de la sphère $\mathbb{S}^{3}$ muni d'une $\Gamma$-structure transverse. Il donne lieu ainsi à un élément de $\left[\mathbb{S}^{3}, B \Gamma\right]$ qui fournit l'équivalence d'homotopie annoncée.

\section{IV.2 Le cas du groupe $F^{\prime}$}

On décrit ici de façon sommaire l'idée utilisé par P. Greenberg pour retrouver, dans le cadre de la géométrie CPP, l'équivalence d'homologie $a$ du théorème IV.1.

Dans la suite du texte, les indices des $n$-uplets $\left(x_{1}, \cdots, x_{n}\right)$ seront définis modulo $n$.

\section{IV.2.a Le modèle de James}

Soit $(X, *)$ un espace topologique pointé supposé connexe. On note $\Sigma X$ la suspension réduite de $X$ :

$$
\Sigma X=\frac{X \times[0 ; 1]}{\{*\} \times[0 ; 1] \coprod X \times\{0 ; 1\}}
$$

La projection d'un élément $(x, t) \in X \times[0 ; 1]$ sera notée $x \wedge t$.

On munit $\coprod_{n \geq 1} X^{n}$ de la relation d'équivalence engendrée par les relations:

$$
\left(x_{1}, \cdots, x_{i-1}, *, x_{i+1}, \cdots, x_{n}\right) \sim\left(x_{1}, \cdots, x_{i-1}, x_{i+1}, \cdots, x_{n}\right)=x^{\hat{i}} \quad \forall i \in<1 ; n>
$$

Le modèle de James de l'espace $X$ est par définition l'espace quotient:

$$
J X=\coprod_{n \in \mathbb{N}} X^{n} / \sim
$$

Il est muni du quotient de la topologie faible. La classe d'un élément $x=\left(x_{i}\right)_{i \in<1 ; n>}$ sera notée $\bar{x}$ ou $\left[x_{1}, \cdots, x_{n}\right]$.

Théorème IV.4. (James [Jam] 1955) Le modèle JX est homotopiquement équivalent à $\Omega \Sigma X$, l'espace des lacets pointés sur la suspension réduite de $X$. 
Cette équivalence d'homotopie est réalisée par une application naturelle $j: J X \rightarrow$ $\Omega \Sigma X$ que nous explicitons rapidement pour $X=\mathbb{S}^{n}$. L'égalité $\Sigma \mathbb{S}^{n}=\mathbb{S}^{n+1}$ permet de plonger $\mathbb{S}^{n}$ dans $\Omega \mathbb{S}^{n+1}$ par l'application $\lambda$ définie par:

$$
\lambda(x): t \in \mathbb{S}^{n} \mapsto x \wedge t \in \Sigma \mathbb{S}^{n}
$$

La figure IV.1 donne l'image du chemin $\lambda(x)$ sur la sphère.

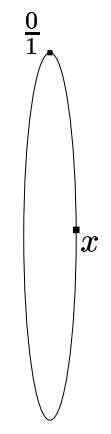

$\mathbb{S}^{1}$

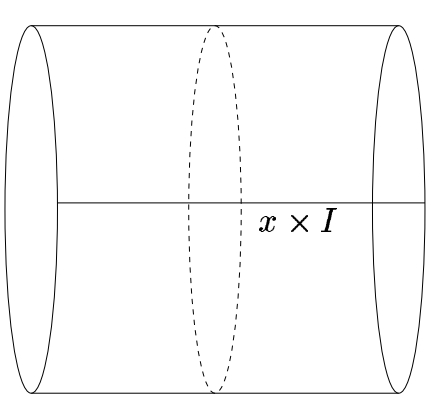

$\mathbb{S}^{1} \times I$

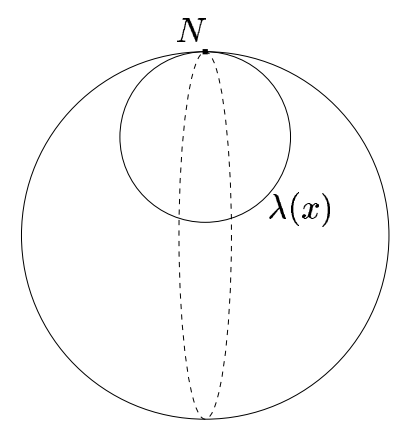

$$
\mathbb{S}^{2}=\mathbb{S}^{1} \times I / \sim
$$

FIG. IV.1 - Le lacet $\lambda(x)$.

On définit alors

$$
j:\left(x_{1}, \cdots, x_{k}\right) \in J \mathbb{S}^{n} \mapsto \lambda\left(x_{k}\right) \circ \cdots \circ \lambda\left(x_{1}\right) \in \Omega \mathbb{S}^{n+1}
$$

où o désigne la composition des chemins. Pour que $j$ soit continue, il faut paramêtrer $\lambda\left(x_{i}\right)$ de sorte que la longueur de ce lacet devienne nulle lorsque $x_{i}$ tend vers le pointbase de $\mathbb{S}^{n}$. On paramêtrise donc $j\left(x_{1}, \cdots, x_{k}\right)$ en stipulant que le lacet $\lambda\left(x_{i}\right)$ est parcouru en un temps $\frac{t_{i}}{\sum_{j=1}^{k} t_{j}}$ où $t_{i}=d_{\mathbb{S}^{n}}\left(x_{i} ; *\right)$.

\section{IV.2.b Une application $\widetilde{\varphi}: B F^{\prime} \rightarrow \Omega \mathbb{S}^{3}$}

Nous définissons d'abord une application $\varphi: B F^{\prime} \rightarrow J \mathbb{S}^{1}$ en utilisant la paramêtrisation horocyclique de $\mathcal{G} r$ définie au corollaire II.3. Soit $n \geq 1$. Considérons l'application

$$
\varphi_{n}: f \in \mathcal{G} r_{n} \mapsto\left(\left[l_{i}\left(f \cdot b_{n}\right)\right]\right)_{i \in<1 ; 3 \cdot 2^{n-1}>} \in\left(\mathbb{S}^{1}\right)^{3 \cdot 2^{n-1}}
$$

où $l_{i}\left(f \cdot b_{n}\right)$ est la $i$-ème longueur horocyclique du bracelet $f \cdot b_{n}$ introduite dans la définition II.2.1. Les prolongements au but $\varphi_{n}: \mathcal{G} r_{n} \rightarrow J \mathbb{S}^{1}$ sont compatibles avec la filtration $\mathcal{G} r_{n} \stackrel{i_{n}}{\subset} \mathcal{G} r_{n+1}$ de $\mathcal{G} r$ et définissent ainsi une application totale $\varphi: \mathcal{G} r \rightarrow J \mathbb{S}^{1}$ invariante par $F^{\prime}$. L'application quotient est aussi notée $\varphi$.

La fibration de Hopf $\mathbb{S}^{1} \rightarrow \mathbb{S}^{3} \rightarrow \mathbb{S}^{2}$ induit la fibration des espaces de lacets $\Omega \mathbb{S}^{1} \rightarrow \Omega \mathbb{S}^{3} \rightarrow \Omega \mathbb{S}^{2}$, dont l'espace total a le type d'homotopie du revêtement universel de $\Omega \mathbb{S}^{2}$. 
Soit $\widetilde{\varphi}: \mathcal{G} r / F^{\prime} \rightarrow \Omega \mathbb{S}^{3}$ un relevé de $\varphi: \mathcal{G} r / F^{\prime}: \rightarrow \Omega \mathbb{S}^{2}$. Dans [Gre4], P.Greenberg donne une esquisse de démonstration du résultat suivant:

Théorème IV.5. L'application $\widetilde{\varphi}$ induit un isomorphisme en homologie entière.

- Remarques:

- Il s'agit bien d'une interprétation de l'application $a$, puisque $\mathcal{G} r / F^{\prime}$ est un $K\left(F^{\prime}, 1\right)$-espace, d'après le corollaire III.1.

- Greenberg utilise le point de vue des bracelets de la géométrie CPP, et notamment le corollaire I.3.

\section{IV.3 Modèle(s) de Cohen de $\Lambda \Sigma X$}

Nous donnons la construction du modèle de Cohen de l'espace des lacets libres sur la suspension réduite d'un espace connexe $X$. Ce modèle se définit à l'aide des configurations d'intervalles du cercle dont nous rappelons la définition. Seul le cas $X=\mathbb{S}^{1}$ (modèle $\Lambda \mathbb{S}^{2}$ ) sera utilisé par la suite.

\section{IV.3.a Configurations ordonnées sur une courbe réelle}

Définition IV.3.1. (Configurations ordonnées)

Une configuration ordonnée de $n$ points du cercle (resp.de $\mathbb{R}$ ) est une suite finie $\left(x_{i}\right)_{i \in<1 ; n>}$ de $\mathbb{S}^{1}(\operatorname{resp} . \mathbb{R})$ vérifiant:

$$
\left.x_{1}<x_{2}<\cdots<x_{n}<x_{1} \text { (resp. } x_{1}<x_{2}<\cdots<x_{n}<x_{1}+1\right)
$$

De même, une suite finie d'intervalles $\left(a_{i}\right)_{i \in<1 ; n>}$ non dégénérés (d'interieurs non vides) de $\mathbb{S}^{1}$ (resp. $\mathbb{R}$ ) est dite configuration ordonnée d'intervalles si ces intervalles sont d'intérieurs deux à deux disjoints et si l'ensemble de ses milieux $\left(m\left(a_{i}\right)\right)_{i \in<1 ; n>}$ forme une configuration ordonnée de points. On définit les ensembles suivants:

$$
\begin{aligned}
F_{0}(n) & =\left\{n-\text { configurations ordonnées de points de } \mathbb{S}^{1}\right\} \\
\widetilde{F_{0}(n)} & =\{n-\text { configurations ordonnées de points de } \mathbb{R}\} \\
F_{0}\left(\mathbb{S}^{1}, n\right) & =\left\{n-\text { configurations ordonnées d'intervalles de } \mathbb{S}^{1}\right\} \\
\widetilde{F_{0}\left(\mathbb{S}^{1}, n\right)} & =\{n-\text { configurations ordonnées d'intervalles de } \mathbb{R}\}
\end{aligned}
$$

Pour deux intervalles $a$ et $b$ de $\mathbb{R}$, on notera $a<b$ si $\stackrel{\circ}{\cap} \cap \circ=\emptyset$ et si $x<y$ pour tout couple d'éléments de $a \times b$. D'autre part, si $r \in \mathbb{R}, a+r$ représente l'intervalle translaté de $a$ par $r$. On notera aussi $r+\left(a_{1}, \cdots, a_{n}\right)=\left(a_{1}+r, \cdots a_{n}+r\right)$ si $r \in \mathbb{R}$. L'espace $F_{0}(n)$ (resp. $\left.\widetilde{F_{0}(n)}\right)$ est muni de la trace de la topologie produit sur $\left(\mathbb{S}^{1}\right)^{n}$ (resp. $\left.\mathbb{R}^{n}\right)$. 
Les espaces $F_{0}\left(\mathbb{S}^{1}, n\right)$ et $\widetilde{F_{0}\left(\mathbb{S}^{1}, n\right)}$ sont munis de la topologie compacte-ouverte, vus comme sous-espaces respectifs de $\mathcal{C}^{0}\left(\coprod_{n} I, \mathbb{S}^{1}\right)$ et de $\mathcal{C}^{0}\left(\coprod_{n} I, \mathbb{R}\right)$.

Le groupe $\mathbb{Z}$ agit sur $\widetilde{F_{0}(n)}$ (resp. $\widehat{F_{0}\left(\mathbb{S}^{1}, n\right)}$ ) en décalant les intervalles (resp. les points) d'un cran vers la droite:

$$
\overline{1} \cdot\left(a_{1}, \cdots a_{n}\right)=\left(a_{2}, \cdots, a_{n}, a_{1}+1\right)
$$

L'espace $F_{0}(n)$ (resp. $\left.F_{0}\left(\mathbb{S}^{1}, n\right)\right)$ a le type d'homotopie du cercle et son revêtement universel est exactement $\widehat{F_{0}(n)}$ (resp. $\left.\widetilde{F_{0}\left(\mathbb{S}^{1}, n\right)}\right)$. Le groupe des automorphismes du revêtement correspond au sous-groupe $n \mathbb{Z} \subset \mathbb{Z}$ de l'action précédente. Notons que cette action donne au quotient une action de $\mathbb{Z} / n \mathbb{Z}$ sur $F_{0}(n)$ et $F_{0}\left(\mathbb{S}^{1}, n\right)$ qui consiste à permuter cycliquement les $n$-configurations ordonnées sur le cercle.

Enfin, les espaces de configurations d'intervalles se surjectent sur ceux des points via l'application qui consiste à prendre les milieux de ces intervalles. Si $a$ est une $n$-configuration ordonnée d'intervalles du cercle (resp. de $\mathbb{R}$ ), on note $M i l^{n}(a)$ (resp. $\left.\widetilde{M i l^{n}}(a)\right)$ la configuration des milieux associés, de sorte que l'on possède le diagramme commutatif suivant:

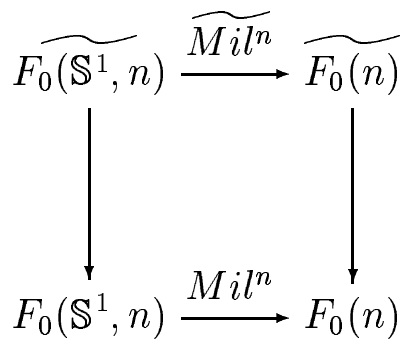

et celui-ci est équivariant pour les actions de $\mathbb{Z}$ en haut et de $\mathbb{Z} / n \mathbb{Z}$ en bas.

\section{IV.3.b Le modèle de $\Lambda \Sigma X$}

Le groupe cyclique $\mathbb{Z} / n \mathbb{Z}$ agit sur le produit $(X)^{n} \times F_{0}\left(\mathbb{S}^{1}, n\right)$ comme suit:

$$
\gamma \cdot(x, a)=\left(x^{\gamma}, a^{\gamma}\right)
$$

où $x^{\gamma}=\left(x_{1+\gamma}, \cdots, x_{\gamma}\right)$ (les indices étant compris modulo $\left.n\right)$. On note

$$
B_{n}(X)=\frac{(X)^{n} \times F_{0}\left(\mathbb{S}^{1}, n\right)}{\mathbb{Z} / n \mathbb{Z}}
$$

où bien $(X)^{n} \times_{\mathbb{Z} / n \mathbb{Z}} F_{0}\left(\mathbb{S}^{1}, n\right)$.

$\mathrm{R}$. Cohen considère l'union disjointe

$$
B(X)=\coprod_{n \in \mathbb{N}^{*}}(X)^{n} \times_{\mathbb{Z} / n \mathbb{Z}} F_{0}\left(\mathbb{S}^{1}, n\right)
$$


munie de la relation d'équivalence engendrée par les relations:

$$
(x, a) \sim \pi_{i}^{n}(x, a)=\left(x_{1}, \cdot, x_{i-1}, x_{i+1}, \cdot, x_{n}, a_{1}, \cdot, a_{i-1}, a_{i+1}, \cdots a_{n}\right)=\left(x^{\hat{i}}, a^{\hat{i}}\right)
$$

lorsque $(x, a) \in A_{i}^{n}=\left\{(x, a) \in B_{n}(X) \mid x_{i}=0\right\}$. Remarquons au passage que les ensembles $\left(A_{i}^{n}\right)_{i \in<1 ; n>}$ ne dépendent pas de $i$ après quotient par $\mathbb{Z} / n \mathbb{Z}$. On note cet ensemble $B_{n}^{0}(X)$. Le quotient de l'union disjointe est noté $L X$ et s'appelle modèle de Cohen (des intervalles) de l'espace $X$. La classe d'un élément $(x, a)$ se note $[x, a]$. Puisque seul le cas $X=\mathbb{S}^{1}$ est utilisé, on notera pour simplifier $B_{n}=B_{n}\left(\mathbb{S}^{1}\right)$ et $B=B\left(\mathbb{S}^{1}\right)$.

Théorème IV.6. (Cohen [Coh] 1985) Soit X un espace connexe. Alors il existe une application naturelle $c: L X \rightarrow \Lambda \Sigma X$ qui fournit une équivalence d'homotopie. Ainsi, l'espace des lacets libres sur la sphère $\mathbb{S}^{2}$ est homotopiquement équivalent au complexe $\coprod\left(\mathbb{S}^{1}\right)^{n} \times_{\mathbb{Z} / n \mathbb{Z}} F_{0}\left(\mathbb{S}^{1}, n\right) / \sim$.

Nous explicitons rapidement l'application $c$. Soit $a=\left(a_{1}, \cdots, a_{n}\right) \in F_{0}\left(\mathbb{S}^{1}, n\right)$. Notons

$$
\pi(a): \mathbb{S}^{1} \rightarrow \bigvee_{i=1}^{n} \mathbb{S}^{1}
$$

l'application qui envoie chaque intervalle $a_{i}$ sur le $i$-ème cercle du bouquet $\vee_{i=1}^{n} \mathbb{S}^{1}$ de manière affine, en préservant l'orientation et de degré 1, et qui envoie tous les points non contenus dans l'union des $a_{i}$ sur le point-base du bouquet. Considérons ensuite

$$
c_{n}: X^{n} \times_{\mathbb{Z} / n \mathbb{Z}} F_{0}\left(\mathbb{S}^{1}, n\right) \rightarrow \Lambda \Sigma X
$$

définie comme étant la composition:

$$
c_{n}([x, a]): \mathbb{S}^{1} \stackrel{\pi(a)}{\longrightarrow} \vee_{i=1}^{n} \mathbb{S}^{1} \stackrel{\vee_{i=1}^{n} \alpha\left(x_{i}\right)}{\longrightarrow} \mathbb{S}^{1} \wedge X=\Sigma X
$$

où $\alpha(x)$ a été définie en IV.2.a. Pour chaque $n$, on a $c_{n}=c_{n-1} \circ \pi_{i \mid A_{i}^{n}}^{n}$ de sorte que l'on peut définir $c: L X \rightarrow \Lambda \Sigma X$ par $c([x, a])=c_{n}(x, a)$ si $(x, a)$ est un représentant de $[x, a]$ dans $B_{n}$. C'est l'application du théorème IV.6.

Remarquons que le modèle $L X$ possède un analogue naturel en remplaçant les configurations d'intervalles en configurations de points. Nous noterons ainsi

$$
\left(B_{n}\right) \bullet(X)=X^{n} \times_{\mathbb{Z} / n \mathbb{Z}} F_{0}(n)
$$

et $(L X)_{\bullet}$, l'analogue de $L X$ pour l'union disjointe des $\left(B_{n}\right)_{\bullet}$. Les applications $\left(M i l^{n}\right)_{n \geq 0}$ sont compatibles avec les applications $\pi_{i}^{n}$ et $\left(\pi_{i}^{n}\right)$. (l'analogue pour les configurations de points) et donnent à la limite $M i l: L X \rightarrow(L X)$. En suivant [Coh], on peut montrer que l'application $M i l$ fournit une équivalence faible d'homotopie. Malheureusement, il semble difficile d'obtenir un inverse global (à homotopie près) explicite de Mil. Les deux modèles seront nécessaires. Celui des intervalles est lié aux espaces de lacets, celui des points à la géométrie CPP. 
Pour finir, on décrit un relevé de l'application composée $y: F_{0}\left(\mathbb{S}^{1}, n\right) \rightarrow \mathbb{S}^{1}$, définie par $y(a)=I(\pi(a)(0))$, où $I: \vee_{i=1}^{n} \mathbb{S}^{1} \rightarrow \mathbb{S}^{1}$ est l'application qui identifie chaque cercle du bouquet isométriquement à un seul cercle, et qui identifie le point-base du bouquet à 0 .

A chaque configuration ordonnée d'intervalles $a=\left(a_{1}, \cdots, a_{n}\right) \in \widetilde{F_{0}\left(\mathbb{S}^{1}, n\right)}$, on note $s_{a}=S u p\left(a_{n}\right)$ et $k_{a}$ le plus grand entier inferieur strictement à $s_{a}$. On définit

$$
r_{a_{i}}^{n}: a_{i} \rightarrow\left[-n k_{a}+i-1 ;-n k_{a}+i\right]
$$

l'unique application affine bijective et croissante de $a_{i}$ sur $\left[-n k_{a}+i-1 ;-n k_{a}+i\right]$. On définit le réel $\tilde{y}(a)$ selon la position de $k_{a}$ par rapport à la configuration d'intervalles $a$. Si $k_{a} \in a_{i}$, on pose $\tilde{y}(a)=r_{a_{i}}^{n}\left(k_{a}\right)$. Si $k_{a}$ est coïncé entre les intervalles $a_{i}$ et $a_{i+1}$, on pose $\tilde{y}(a)=-n k_{a}+i$.

Lemme IV.1. L'application $\tilde{y}: \widetilde{F_{0}\left(\mathbb{S}^{1}, n\right)} \rightarrow \mathbb{R}$ est continue et donne le diagramme commutatif suivant:

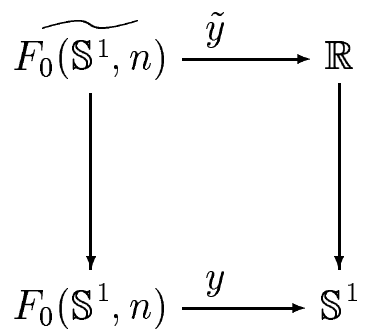

Si $\gamma$ est le générateur de l'action de $\mathbb{Z}$ sur $\widehat{F_{0}\left(\widetilde{\mathbb{S}^{1}}, n\right)}$ par décalage des intervalles d'un cran vers la droite, on a l'égalité $\tilde{y}(\gamma \cdot a)=\tilde{y}(a)-1$.

Preuve. - Le point intéressant de la continuité se trouve lorsque $s_{a}$ se rapproche supérieurement de $k_{a}$. Dans ce cas, $\tilde{y}(a)=r_{a_{n}}^{n}\left(k_{a}\right)$ converge vers $-n k_{a}+n$ par construction de $r_{a_{n}}^{n}$. Notons $a^{\prime}$ une configuration limite de sorte que $k_{a^{\prime}}=k_{a}-1$ par définition de $k_{a}$ et de $k_{a^{\prime}}$. Puisque $k_{a^{\prime}}$ est coïncé entre les intervalles $a_{n}^{\prime}-1$ et $a_{1}^{\prime}$, on a $\tilde{y}\left(a^{\prime}\right)=-n k_{a^{\prime}}=-n k_{a}+n$ ce qui montre que $\tilde{y}(a)$ tend vers $\tilde{y}\left(a^{\prime}\right)$.

La commutativité du diagramme et la derniére égalité proviennent des faits suivants: l'application $\pi(a)$ envoie un intervalle $a_{i}$ de manière affine et de degré 1 sur le cercle unité de sorte que les extrémités de $a_{i}$ sont envoyées sur 0 et l'application $r_{a_{i}}^{n}$ envoie $a_{i}$ de manière affine sur un intervalle de longueur 1 de sorte que les extrémités de $a_{i}$ sont envoyées sur deux entiers consécutifs. On conclut en remarquant qu'étant donné deux intervalles réels $A$ et $B$, il existe une unique transformation affine croissante envoyant bijectivement $A$ sur $B$.

\section{IV.4 Un nouveau modèle de $\Lambda \mathbb{S}^{3}$}

Dans la suite du texte, un $\mathbb{S}^{1}$-espace principal (où succintement $\mathbb{S}^{1}$-espace) désigne un espace topologique muni d'une action continue et libre du cercle. Un $\mathbb{S}^{1}$-fibré principal $E \longrightarrow B$ (où simplement $\mathbb{S}^{1}$-fibré, voire fibré en cercles) est un $\mathbb{S}^{1}$-espace 
principal localement trivial, où de façon équivalente, $E \longrightarrow B$ admet une section locale en tout point de $B$. Un morphisme d'espaces principaux est par définition une application continue $\mathbb{S}^{1}$-équivariante. Enfin, si $E \rightarrow B$ est un fibré principal et $B^{\prime} \stackrel{c}{\rightarrow} B$ une application continue, le pull-back de $E$ par $c$ est noté $E_{c}$, ou bien $B^{\prime} \times_{B} E$.

\section{IV.4.a Stratégie de construction}

Afin de capter entièrement l'homologie du groupe $T$, nous souhaitons construire une application de $B \widetilde{T}$ vers $\Lambda \mathbb{S}^{3}$. L'application de Greenberg de $B F^{\prime}$ vers $\Omega \mathbb{S}^{3}$ indique que le modèle de Cohen appliqué à $\mathbb{S}^{2}$ est mieux adapté à la géométrie des bracelets que le modèle appliqué à $\mathbb{S}^{3}=\Sigma \mathbb{S}^{2}$. Cependant, $\Lambda \mathbb{S}^{3}$ n'est pas le revêtement universel de $\Lambda \mathbb{S}^{2}$.

Notons les applications d'évaluation au point-base de $\mathbb{S}^{1}$ :

$$
e v^{i}: f \in \Lambda \mathbb{S}^{i} \mapsto f(0) \in \mathbb{S}^{i} \quad i \in\{2 ; 3\}
$$

La fibration de Hopf $h: \mathbb{S}^{3} \rightarrow \mathbb{S}^{2}$ donne lieu à l'application $H: f \in \Lambda \mathbb{S}^{3} \mapsto h \circ f \in \Lambda \mathbb{S}^{2}$. Ces quatre applications fournissent le diagramme commutatif suivant:

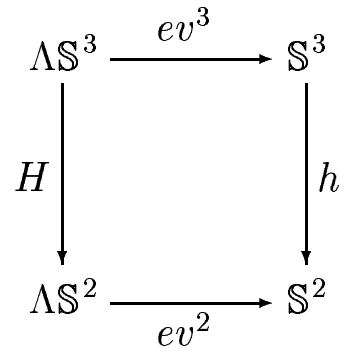

qui factorise en la composée

$$
\Lambda \mathbb{S}^{3} \stackrel{\left(H, e v^{3}\right)}{\longrightarrow} \Lambda \mathbb{S}^{2} \times \mathbb{S}^{2} \mathbb{S}^{3} \stackrel{h=e v^{2}}{\longrightarrow} \mathbb{S}^{2}
$$

où

$$
\Lambda \mathbb{S}^{2} \times_{\mathbb{S}^{2}} \mathbb{S}^{3}=\left\{(f, x) \in \Lambda \mathbb{S}^{2} \times \mathbb{S}^{3} \mid f(0)=h(x)\right\} \subset \Lambda \mathbb{S}^{2} \times \mathbb{S}^{3}
$$

est le pullback du fibré de Hopf par $e v^{2}$. Il s'agit donc d'un $\mathbb{S}^{1}$-fibré principal au dessus de $\Lambda \mathbb{S}^{2}$, dont le revêtement universel n'est autre que $\Lambda \mathbb{S}^{3}$ et a pour groupe de revêtement $\mathbb{Z}$.

Notre stratégie est donc la suivante. On détermine un modèle $K \mathbb{S}^{2}$ pour l'espace $\Lambda \mathbb{S}^{2} \times_{\mathbb{S}^{2}} \mathbb{S}^{3}$, en relevant en quelque sorte le modèle de Cohen $L \mathbb{S}^{1}$ de $\Lambda \mathbb{S}^{2}$. Comme pour ce dernier, nous développons deux versions de $K \mathbb{S}^{2}$, en utilisant configurations ordonnées de points et d'intervalles sur $\mathbb{R}$. En passant au revêtement universel, on en déduit rapidement un modèle de $\Lambda \mathbb{S}^{3}$.

L'idée de la construction de $K \mathbb{S}^{2}$ repose sur les $\mathbb{S}^{1}$-fibrés $\left(\mathcal{H}_{n}\right)_{n \geq 1}$ au dessus des tores $\left(\mathbb{T}^{2 n}\right)_{n \geq 1}$, construis à partir des groupes de Heisenberg de dimension paire. Une version combinatoire du fibré de Hopf dont nous donnons maintenant la construction, provient du fibré $\mathcal{H}_{1}$. 


\section{IV.4.b Du fibré de Heisenberg au fibré de Hopf}

Notons $\left(H_{2 n+1}, *\right)$ le groupe de Heisenberg réel. Il s'agit de l'espace $\mathbb{R}^{n} \times \mathbb{R}^{n} \times \mathbb{R}$ muni du produit:

$$
(x, y, z) *(p, q, r):=(x+p, y+q, z+r+x \cdot q)
$$

où $x \cdot q$ représente le produit scalaire des vecteurs $x$ et $q$. Le réseau des entiers $Z_{2 n+1}=\mathbb{Z}^{n} \times \mathbb{Z}^{n} \times \mathbb{Z}$ (noté de cette façon pour ne pas faire de confusion avec le groupe commutatif $\mathbb{Z}^{2 n+1}$ ) est un sous-groupe discret de $H_{2 n+1}$. L'espace des classes d'équivalence à droite $\mathcal{H}_{n}=H_{2 n+1} / Z_{2 n+1}$ est la variété de Heisenberg. Remarquons que $\mathcal{H}_{n}$ est un $\mathbb{S}^{1}$-fibré au dessus du tore $\mathbb{T}^{2 n}$, non trivial car

$$
\Pi_{1}\left(\mathbb{T}^{2 n} \times \mathbb{S}^{1}\right)=\mathbb{Z}^{2 n+1} \neq Z_{2 n+1}=\Pi_{1}\left(\mathcal{H}_{n}\right)
$$

L'action sur les fibres s'effectue par translation sur le facteur $z$.

Le modèle de Cohen utilise la suspension réduite du cercle que l'on verra maintenant comme le quotient:

$$
\Sigma \mathbb{S}^{1}=\mathbb{S}^{2}=\mathbb{T}^{2} / \Xi
$$

où $\Xi=\{0\} \times \mathbb{S}^{1} \amalg \mathbb{S}^{1} \times\{0\}$ est le bouquet de cercle à deux pétales plongé dans le tore. On notera $\pi: \Xi \rightarrow\{0\} \times\{0\}$ et $e c:(x, y) \in \mathbb{T} \rightarrow x \wedge y \in \Sigma \mathbb{S}^{1}$ l'application de recollement (ou écrasement, d'où la notation) associée. Nous construisons un fibré $\widehat{\Sigma \mathbb{S}^{1}} \rightarrow \Sigma \mathbb{S}^{1}$ à partir du fibré de Heisenberg $\mathcal{H}_{1} \rightarrow \mathbb{T}^{2}$ en identifiant isomorphiquement les fibres au-dessus des génératrices de $\mathbb{T}^{2}$.

Soit $(m, n) \in \mathbb{Z}^{2}, U_{m}=\{m\} \times \mathbb{R}^{2}$ et $V_{n}=\mathbb{R} \times\{n\} \times \mathbb{R}$ de sorte que

$$
\widetilde{C_{0}}=\cup_{(m, n) \in \mathbb{Z}^{2}} U_{m} \cup V_{n} \subset \mathbb{R}^{3}
$$

est invariant par $Z_{3}$ et donne au quotient $C_{0}=H e^{-1}(\Xi)$. Considérons l'application:

$$
\begin{array}{rccc}
\widetilde{\Pi}: & \widetilde{C_{0}} & \rightarrow & \mathbb{S}_{0}^{1} \\
(m, y, z) \in U_{m} & \mapsto & \widetilde{\Pi_{1}}(m, y, z)=[z] \\
(x, n, z) \in V_{n} & \mapsto & \widetilde{\Pi}_{2}(x, n, z)=[z-n x]
\end{array}
$$

Celle-ci est bien définie puisque $\widetilde{\Pi_{1}}$ et $\widetilde{\Pi_{2}}$ coïncident sur $U_{m} \cap V_{n}$. De plus, pour tout $Y_{m}=(m, y, z) \in U_{m}, X_{n}=(x, n, z) \in V_{n}$ et $A=(a, b, c) \in Z_{3}$, on a les égalités dans $\mathbb{R}^{3}$ :

$$
\begin{gathered}
\widetilde{\Pi_{1}}\left(Y_{m}\right) * A=\widetilde{\Pi_{1}}\left(Y_{m} * A\right) *(0,0,-b m) \\
\widetilde{\Pi_{2}}\left(X_{n}\right) * A=\widetilde{\Pi_{2}}\left(X_{n} * A\right) *(a, b, a b+a m)
\end{gathered}
$$

qui entrainent la $Z_{3}$-invariance de $\widetilde{\Pi}$. Notons $\Pi: C_{0} \rightarrow \mathbb{S}_{0}^{1}$ l'application quotient. Considérons alors l'espace $\widehat{\Sigma \mathbb{S}^{1}}$ construit en recollant $\mathbb{S}_{0}^{1}$ à $\mathcal{H}_{1}$ par $\Pi$, c'est à dire:

$$
\widehat{\Sigma \mathbb{S}^{1}}=\frac{\mathcal{H}_{1} \coprod \mathbb{S}_{0}^{1}}{\left\{X \sim \Pi(X), X \in C_{0}\right\}}=\mathcal{H}_{1} \coprod_{\Pi} \mathbb{S}_{0}^{1}
$$


On note $E C: \mathcal{H}_{1} \rightarrow \widehat{\Sigma \mathbb{S}^{1}}$ l'application de recollement associée. Si $X$ et $Y$ sont deux éléments de $\mathcal{H}_{1}$ ayant même image par $\widetilde{\Pi}$, alors ils ont même image par $\pi \circ \mathrm{He}$. Ceci permet de définir une application quotient $\widehat{H e}: \widehat{\Sigma \mathbb{S}^{1}} \rightarrow \Sigma \mathbb{S}^{1}$ vérifiant le diagramme commutatif:

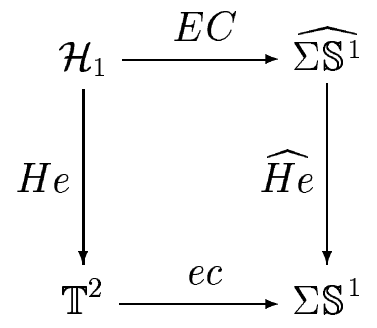

Lemme IV.2. L'application $\widehat{H e}: \widehat{\Sigma \mathbb{S}^{1}} \rightarrow \Sigma \mathbb{S}^{1}$ est la projection d'un $\mathbb{S}^{1}$-fibré principal isomorphe au fibré de Hopf.

Preuve. - Puisque l'application $\Pi$ est $\mathbb{S}^{1}$-équivariante, l'action du cercle sur $\mathcal{H}_{1}$ passe au quotient en une action libre sur $\widehat{\Sigma \mathbb{S}^{1}}$. C'est donc un $\mathbb{S}^{1}$-espace principal et l'application $E C$ est un morphisme d'espaces principaux. Il reste à montrer que $\widehat{\Sigma \mathbb{S}^{1}}$ est localement trivial.

Remarquons que l'application $E C$ est un homéomorphisme $\mathbb{S}^{1}$-équivariant en restriction au complémentaire de $C_{0}$, et d'image le complémentaire de la fibre au dessus du point-base $N$ de $\Sigma \mathbb{S}^{1}$. Il fournit ainsi une trivialisation de $\widehat{\Sigma \mathbb{S}^{1}}$ sur le complémentaire de $N$ dans $\Sigma \mathbb{S}^{1}$. Il suffit donc de vérifier la trivialité au voisinage de $N$.

Celle-ci provient d'une trivialisation locale de $\mathcal{H}_{1}$ sur un voisinage de $\Xi$ compatible avec les relations $\pi$ et $\Pi$ que nous construisons maintenant. L'application $\varphi_{\Xi}=(H e, \Pi): H e^{-1}(\Xi) \rightarrow \Xi \times \mathbb{S}_{0}^{1}$ définit une trivialisation du fibré $\mathcal{H}_{1}$ au dessus de $\Xi$. Elle vérifie de plus la relation de compatibilité avec les recollements: $\varphi_{\Xi} \circ \Pi=\pi \circ \varphi_{\Xi}$. Soit $U$ un voisinage ouvert de $\Xi$ qui se rétracte par déformation sur $\Xi$, de sorte que l'on peut prolonger $\varphi_{\Xi}$ en un homéomorphisme $\mathbb{S}^{1}$-équivariant $\varphi_{U}: H e^{-1}(U) \rightarrow U \times \mathbb{S}^{1}$. Celui-ci est compatible avec $\Pi$ et $\pi$ puisqu'il coïncide avec $\varphi_{\Xi}$ sur $H e^{-1}(\Xi)$ qui est exactement le "support" de $\Pi$. Il passe donc aux quotients pour donner un $\mathbb{S}^{1}$-isomorphisme entre $E C(U)$ et $e c(U) \times \mathbb{S}^{1}$. C'est une trivialisation locale au voisinage du point-base de $\Sigma \mathbb{S}^{1}$.

On en déduit que $\widehat{\Sigma \mathbb{S}^{1}}$ est un $\mathbb{S}^{1}$-fibré. L'application $e c$ induit un isomorphisme entre $H_{2}\left(\mathbb{T}^{2}\right)$ et $H_{2}\left(\Sigma \mathbb{S}^{1}\right)$. Notons $\sigma$ (resp. $\gamma$ ) le générateur de $H_{2}\left(\mathbb{T}^{2}\right)\left(\right.$ resp. $H_{2}\left(\Sigma \mathbb{S}^{1}\right)$ ). Par naturalité de la classe d'Euler, on obtient:

$$
<e\left(\widehat{\Sigma \mathbb{S}^{1}}\right), \gamma>=<e\left(\mathcal{H}_{1}\right), \sigma>=1
$$

On en déduit que les classes d'Euler des fibrés $\widehat{\Sigma \mathbb{S}^{1}}$ et $\mathbb{S}^{3}$ coïncident. Ces deux fibrés en cercle sont donc isomorphes. 


\section{IV.4.c Le modèle de $\Lambda \mathbb{S}^{2} \times_{\mathbb{S}^{2}} \mathbb{S}^{3}$}

Dans la suite du texte, si $r \in \mathbb{R}$, on note $r_{n}=(r, r, \cdots, r) \in \mathbb{R}^{n}$.

Commençons par l'étude de $\Lambda \mathbb{S}^{2} \times_{\mathbb{S}^{2}} \mathbb{S}^{3}$. On remarque d'abord que pour chaque $n$, l'espace $B_{n}=\left(\mathbb{S}^{1}\right)^{n} \times_{\mathbb{Z} / n \mathbb{Z}} F_{0}(n)$ est inclus dans le quotient du tore $\left(\mathbb{S}^{1}\right)^{n} \times\left(\mathbb{S}^{1}\right)^{n}$ par l'action diagonale de $\mathbb{Z} / n \mathbb{Z}$. Un $\mathbb{S}^{1}$-fibré naturel et non trivial au dessus de $\mathbb{T}^{2 n}$ est la variété de Heisenberg $\mathcal{H}_{n}$. On va par conséquent construire un modèle de $\Lambda \mathbb{S}^{2} \times_{\mathbb{S}^{2}}$ $\mathbb{S}^{3}$ composé de morceaux ressemblant aux variétés de Heisenberg, recollés par des applications de projections, à l'instar du modèle de Cohen.

Une sous-variété de $\mathcal{H}_{n}$ tordue par $\mathbb{Z} / n \mathbb{Z}$

Considérons à présent

$$
H_{2 n+1}^{+}=\mathbb{R}^{n} \times \widetilde{F_{0}(n)} \times \mathbb{R}
$$

Le sous-groupe

$$
Z_{n+2}^{+}=\left\{(x, y, z) \in Z_{2 n+1} \mid y_{i}=y_{j} \forall(i, j)\right\}
$$

de $Z_{2 n+1}$ agit proprement discontinument et librement par multiplication à droite sur $H_{2 n+1}^{+}$et donne une variété lisse au quotient.

Définition IV.4.1. On note $\mathcal{K}_{n}$ le quotient de $H_{2 n+1}^{+}$par $Z_{n+2}^{+}$.

On possède une action de $\mathbb{Z} / n \mathbb{Z}$ sur $\mathcal{K}_{n}$ qui est la suivante. Considérons l'application:

$$
\begin{aligned}
& \gamma_{n}: H_{2 n+1}^{+} \quad \rightarrow \quad H_{2 n+1}^{+} \\
& (x, y, z) \mapsto\left(\left(x_{2}, \cdots, x_{n}, x_{1}\right),\left(y_{2}, \cdots, y_{n}, y_{1}+1\right), z+x_{1}\right)
\end{aligned}
$$

Un calcul simple montre que pour $X=(x, y, z) \in H_{2 n+1}^{+}$et $A=(p, q, r) \in Z_{n+2}^{+}$on a l'égalité:

$$
\gamma_{n}(X * A)=\gamma_{n}((x, y, z) *(p, q, r))=\gamma_{n}(x, y, z) *\left(p^{\overline{1}}, q, r\right)
$$

où $\overline{1}$ est la classe de 1 modulo $n$.

Ainsi, l'application $\gamma_{n}$ passe au quotient en une application, toujours notée $\gamma_{n}$ de $\mathcal{K}_{n}$ dans lui-même. La condition IV.1 implique que $\gamma_{n}$ est d'ordre au moins $n$. De plus, on a l'égalité:

$$
\gamma_{n}^{n}(x, y, z)=(x, y, z) *\left(0_{n}, 1_{n}, 0\right)
$$

ce qui montre que $\gamma_{n}^{n}$ est l'identité sur l'espace $\mathcal{K}_{n}$. L'application $\gamma_{n}$ fournit l'action suivante:

$$
\begin{array}{ccccc}
\mathbb{Z} / n \mathbb{Z} & \times & \mathcal{K}_{n} & \rightarrow & \mathcal{K}_{n} \\
(\bar{i} & , & X) & \mapsto & \bar{i} \cdot X=\gamma_{n}^{i}(X)
\end{array}
$$

Définition IV.4.2. On note $E_{n}$ le quotient de $\mathcal{K}_{n}$ par l'action de $\mathbb{Z} / n \mathbb{Z}$.

Cet espace est muni de la topologie quotient. 


\section{Recollement des sous-variétés tordues}

Nous sommes en mesure de construire le modèle de $\Lambda \mathbb{S}^{2} \times_{\mathbb{S}^{2}} \mathbb{S}^{3}$ en se plaçant sur l'union disjointe

$$
K=\coprod_{n \geq 1} E_{n}
$$

On munit cet espace de la relation d'équivalence $\sim$ engendrée par les relations $\sim_{i}^{n}$ définies comme suit.

Si $x=\left(x_{1}, \cdots, x_{i-1}, 0, x_{i+1}, \cdots, x_{n}\right)$, et $X=(x, y, z) \in E_{n}$, on pose:

$$
(x, y, z) \sim_{i}^{n}\left(x^{\hat{i}}, y^{\hat{i}}, z\right):=\Pi_{n}^{i}(X)
$$

où $\left(x^{\hat{i}}, y^{\hat{i}}, z\right)$ est un élément de $E_{n-1}$.

Définition IV.4.3. (Modèle combinatoire de $\Lambda \mathbb{S}^{2} \times_{\mathbb{S}^{2}} \mathbb{S}^{3}$ )

On note:

$$
K \mathbb{S}^{2}=K / \sim
$$

et $p$ la projection de $K$ sur $K \mathbb{S}^{2}$. La classe d'équivalence de $(x, y, z) \in K$ sera notée $[x, y, z]$.

Notons enfin les égalités, pour $X=[x, y, z]$ vérifiant $x_{i}=0$,

$$
\Pi_{n}^{i-1}\left(\gamma_{n} \cdot X\right)=\gamma_{n-1} \cdot \Pi_{n}^{i}(X) \text { si } i \geq 2
$$

et

$$
\Pi_{n}^{n}\left(\gamma_{n} \cdot X\right)=\Pi_{n}^{1}(X) *(0,0,-1) \text { si } i=1
$$

Cette remarque nous permet dans les calculs de quotienter et de faire agir des permutations cycliques sans se soucier du sens dans lequel s'effectue cette double opération.

On munit $K$ de la topologie faible et $K \mathbb{S}^{2}$ de la topologie quotient. Nous avons ainsi défini un espace topologique combinatoire utilisant $\widehat{F_{0}(n)}$. La même manipulation en remplaçant $\widetilde{F_{0}(n)}$ par $\widetilde{F_{0}\left(\mathbb{S}^{1}, n\right)}$ conduit à un autre espace combinatoire. Pour distinguer ces deux modèles, les espaces et les applications relatifs au modèle des points seront indexés par un point gras en bas. Par exemple, on note $\left(H_{2 n+1}^{+}\right)_{\bullet}=$ $\mathbb{R}^{n} \times \widetilde{F_{0}(n)} \times \mathbb{R}$ et $H_{2 n+1}^{+}=\mathbb{R}^{n} \times \widehat{F_{0}\left(\mathbb{S}^{1}, n\right)} \times \mathbb{R}$.

Le fibré combinatoire $\Lambda \mathbb{S}^{2} \times_{\mathbb{S}^{2}} \mathbb{S}^{3} \rightarrow \Lambda \mathbb{S}^{2}$

Soit $n \geq 1$. On possède les projections suivantes:

$$
\begin{gathered}
p_{n}:(x, \tilde{a}, z) \in H_{2 n+1}^{+} \mapsto[x, a] \in\left(\mathbb{S}^{1}\right)^{n} \times F_{0}\left(\mathbb{S}^{1}, n\right) \\
\left(p_{n}\right)_{\bullet}:(x, y, z) \in\left(H_{2 n+1}^{+}\right) \bullet \mapsto[x, y] \in\left(\mathbb{S}^{1}\right)^{n} \times F_{0}(n)
\end{gathered}
$$


Proposition IV.1. L'application $p: K \mathbb{S}^{2} \rightarrow L \mathbb{S}^{1}\left(\right.$ resp. $p_{\bullet}:\left(K \mathbb{S}^{2}\right)$.) définie par $p(X)=p_{n}(X)$ (resp. $p_{\bullet}(X)=\left(p_{n}\right) \cdot(X)$ si $X \in E_{n}$ (resp. $X \in\left(E_{n}\right)$. est définie et continue.

Preuve. $-\mathrm{Si}(X, A) \in H_{2 n+1}^{+} \times Z_{n+2}^{+}$, alors on a par définition de $p_{n}$ de la loi $*$, et $\gamma_{n}$, les égalités $p_{n}(X * A)=p_{n}(X)$ et $p_{n}\left(\gamma_{n} \cdot X\right)=\gamma_{n} \cdot p_{n}(X)$. Par conséquent, $p_{n}$ passe aux quotients en une application continue $p_{n}: E_{n} \rightarrow B_{n}$.

Les applications $p_{n}$ sont compatibles avec les relations d'équivalence $\pi_{n}^{i}$ en bas et $\Pi_{n}^{i}$ en haut. En effet, si $x_{i}=0$, on a l'égalité $p_{n+1}\left(\Pi_{n}^{i}(X)\right)=\pi_{n}^{i}\left(p_{n}(X)\right)$. L'application $p$ est donc bien définie. De plus, chaque application $p_{n}$ est continue et puisque $K \mathbb{S}^{2}$ et $L \mathbb{S}^{1}$ sont munis de la topologie faible, l'application $p$ est continue. Les mêmes arguments s'appliquent à $p_{\text {. }}$.

Notons que les applications $\left(M i l^{n}\right)_{n \geq 0}$ fournissent le diagramme commutatif suivant:

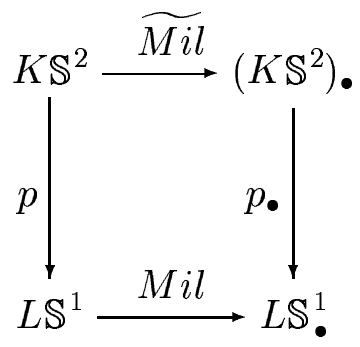

On relie maintenant le modèle des intervalles $K \mathbb{S}^{2}$ à l'espace $\Lambda \mathbb{S}^{2} \times_{\mathbb{S}^{2}} \mathbb{S}^{3}$, via le fibré $\widehat{\Sigma \mathbb{S}^{1}}$. Soit $X=(x, a, z) \in H_{2 n+1}^{+}$tel que $k_{a} \in a_{i}\left(k_{a}\right.$ a été défini à la fin de IV.3.b) et posons

$$
\widehat{d_{n}}(X)=\left[x_{i}, \tilde{y}(a),-z+k_{a} \sum_{k=1}^{n}\left[x_{k}-x_{i}\right]+\sum_{k=1}^{i-1}\left[x_{i}-x_{k}\right]\right]
$$

Notons $a_{i}^{+}$(resp. $a_{i}^{-}$) la borne supérieure (resp. inférieure) de l'intervalle $a_{i}$. La définition de $\widetilde{\Pi_{2}}$ et la continuité de la fonction $\tilde{y}$ impliquent que $\widehat{d_{n}}(X)$ converge vers $\left[0,0,-z+k_{a} \sum_{k=1}^{n} x_{k}-\sum_{k=1}^{i} x_{k}\right]$ lorsque $a_{i}^{+}$tend vers $k_{a}$, mais aussi lorsque $a_{i+1}^{-}$ tend vers $k_{a}$. On pose donc

$$
\widehat{d_{n}}(X)=\left[0,0,-z+k_{a} \sum_{k=1}^{n} x_{k}-\sum_{k=1}^{i} x_{k}\right]
$$

si l'entier $k_{a}$ est dans l'intervalle $\left[a_{i}^{+} ; a_{i+1}^{-}\right]$.

Lemme IV.3. L'application $\widehat{d_{n}}$ est continue sur $H_{2 n+1}^{+}$et passe aux quotients successifs par $Z_{n+2}^{+}$et $\mathbb{Z} / n \mathbb{Z}$. Pour tout couple $(n, i) \in \mathbb{N}^{*} \times\langle 1 ; n\rangle$, on a l'égalité $\widehat{d_{n-1}} \circ \Pi_{i}^{n}=\widehat{d_{n}}$, ce qui fournit une application continue $\widehat{d}: K \mathbb{S}^{2} \rightarrow \widehat{\Sigma \mathbb{S}^{1}}$ donnant le 
diagramme commutatif:

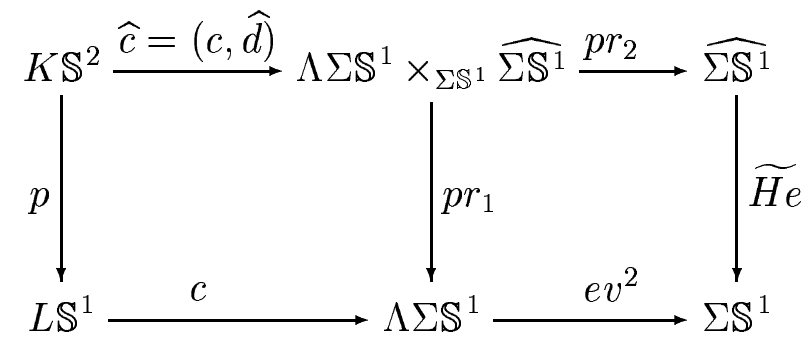

Preuve. - Par construction, l'application $\widehat{d}_{n}$ est continue sauf éventuellement aux points $X=(x, a, z)$ vérifiant $s_{a}=\operatorname{Sup}\left(a_{n}\right) \in \mathbb{Z}$. Le point intéressant se trouve encore lorsque $s_{a}$ tend vers un entier $p$ supérieurement. Si $s_{a}=p+\varepsilon$ avec $\varepsilon$ positif et proche de 0 , on a $k_{a}=p \in a_{n}$. On a donc

$$
\widehat{d_{n}}(X)=\left[x_{n}, \tilde{y}(a),-z+p \sum_{k=1}^{n}\left[x_{k}-x_{n}\right]+\sum_{k=1}^{n-1}\left[x_{n}-x_{k}\right]\right]
$$

Lorsque $\varepsilon$ tend vers 0 , l'expression de droite tend vers $\left[0,0,-z+(p-1) \sum_{k=1}^{n} x_{k}\right]$. Lorsque $s_{a}=p$, on a $k_{a}=s_{a}-1=p-1$, et dans ce cas, on a

$$
\widehat{d_{n}}(X)=\left[0,0,-z+(p-1) \sum_{k=1}^{n} x_{k}\right]
$$

ce qui montre la continuité de $\widehat{d_{n}}$.

En utilisant essentiellement les égalités $\tilde{y}(a+q)=\tilde{y}(a)-n q, k_{a+q}=k_{a}+q$ pour $q \in \mathbb{Z}$ et la projection $\widetilde{\Pi_{2}}$, on obtient sans difficulté l'invariance de $\widehat{d}_{n}$ par l'action de $Z_{n+2}^{+}$. De même, l'égalité $\tilde{y}(\gamma \cdot a)=\tilde{y}(a)-1$ (où $\gamma$ est générateur de $\mathbb{Z} / n \mathbb{Z}$ ) entraine l'invariance par $\mathbb{Z} / n \mathbb{Z}$.

La relation $\widehat{d_{n-1}} \circ \Pi_{i}^{n}=\widehat{d_{n}}$ provient de la définition de $\widetilde{\Pi_{1}}$. Enfin, la commutativité des diagrammes est une conséquence de celle du diagramme énoncé au lemme IV.1.

Corollaire IV.1. $K \mathbb{S}^{2}$ est l'espace total d'un $\mathbb{S}^{1}$-fibré principal au dessus de $L \mathbb{S}^{1}$.

Notons que $\Lambda \mathbb{S}^{2} \times_{\mathbb{S}^{2}} \mathbb{S}^{3} \rightarrow \Lambda \mathbb{S}^{2}$ est isomorphe au fibré $\Lambda \Sigma \mathbb{S}^{1} \times_{\Sigma \mathbb{S}^{1}} \widehat{\Sigma \mathbb{S}^{1}} \rightarrow \Lambda \Sigma \mathbb{S}^{1}$. L'action de la fibre sur $\Lambda \mathbb{S}^{2} \times_{\mathbb{S}^{2}} \mathbb{S}^{3}$ (resp. sur $\Lambda \Sigma \mathbb{S}^{1} \times_{\Sigma \mathbb{S}^{1}} \widehat{\Sigma \mathbb{S}^{1}}$ ) se fait uniquement sur $\mathbb{S}^{3}$ par l'action sur le fibré de Hopf (resp. sur $\widehat{\Sigma \mathbb{S}^{1}} \operatorname{par}[s] \cdot[x, y, z]=[x, y, z-s]$ ).

Preuve. - Quitte à considérer l'action du cercle $[x, y, z] \cdot[s]=[x, y, z-s]$ sur $\widehat{\Sigma \mathbb{S}^{1}}$, on peut supposer que les applications $\widehat{d_{n}}$ et $\widehat{d}$ sont $\mathbb{S}^{1}$-équivariantes. Elles fournissent donc des isomorphismes en restriction aux fibres $p_{n}^{-1}(x)$ (resp. $p^{-1}(x)$ ). 
Fixons $n \geq 0$. Puisque $\widehat{H e} \circ \widehat{d_{n}}=c_{n} \circ p_{n}$, on a le diagramme suivant:

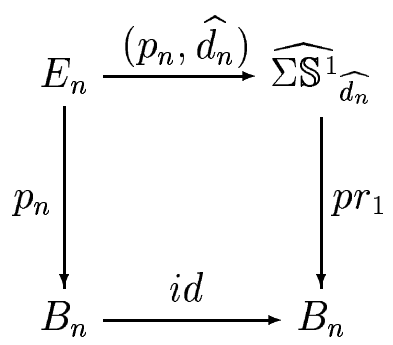

où $p r_{1}$ est la projection sur le premier facteur. La flèche de droite est un $\mathbb{S}^{1}$-fibré et nous souhaitons qu'il en soit de même pour celle de gauche. Considérons une trivialisation locale de $\widehat{\Sigma \mathbb{S}^{1}}{\widehat{d_{n}}}_{\text {. Soit }} U_{n} \subset B_{n}$ un ouvert trivialisant du fibré pull-back $\widehat{\Sigma \mathbb{S}^{1}}{\widehat{d_{n}}}_{\text {. Il }}$. existe un homéomorphisme $\mathbb{S}^{1}$-équivariant $\phi_{U_{n}}: p r_{1}^{-1}\left(U_{n}\right) \rightarrow U_{n} \times \mathbb{S}^{1}$. Le diagramme

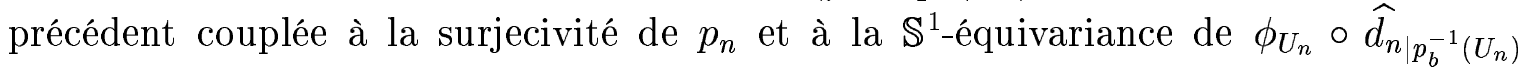
entraine que $\phi_{U_{n}} \circ \widehat{d_{n}}: p_{n}^{-1}\left(U_{n}\right) \rightarrow U_{n} \times \mathbb{S}^{1}$ est un homéomorphisme tel que $p r_{1} \circ\left(\phi_{U_{n}} \circ\right.$ $\left.\widehat{d_{n}}\right)=p_{n}$, de sorte que c'est une trivialisation $\mathbb{S}^{1}$-équivariante de $E_{n}$ au dessus de $U_{n}$. On en déduit que $E_{n}$ est un $\mathbb{S}^{1}$-fibré principal au dessus de $B_{n}$.

Pour montrer que $K \mathbb{S}^{2}$ est un $\mathbb{S}^{1}$-fibré principal, on procède de manière analogue. Il faut considérer un ouvert trivialisant $U=\coprod U_{n} / \sim$ du fibré $\left(\Lambda \Sigma \mathbb{S}^{1} \times_{\Sigma \mathbb{S}^{1}} \widehat{\Sigma \mathbb{S}^{1}}\right)_{\widehat{c}}$, tel que chaque $U_{n}$ soit trivialisant pour le fibré $E_{n}$. On conclut grâce à la $\mathbb{S}^{1}$-équivariance des applications de recollement $\Pi_{n}^{i}$.

Le résultat suivant peut se montrer directement en utilisant les suites exactes en homotopie associées aux fibrés $K \mathbb{S}^{2}$ et $\Lambda \mathbb{S}^{2} \times_{\mathbb{S}^{2}} \mathbb{S}^{3}$. En effet, l'application de Cohen $c$ est une équivalence d'homotopie. Le lemme du serpent permettrait de conclure que $\hat{c}$ l'est également. Nous préférons cependant donner une preuve invoquant les classes d'Euler de chacuns des deux fibrés. La motivation de cette preuve est le point 3. que l'on utilisera pour montrer (corollaire IV.5) la nullité du cocycle $\mu$ introduit dans la proposition IV.4.

Théorème IV.7. L'espace total du fibré en cercle $K \mathbb{S}^{2} \rightarrow L \mathbb{S}^{1}$ est faiblement homotopiquement équivalent à celui de $\Lambda \mathbb{S}^{2} \times_{\mathbb{S}^{2}} \mathbb{S}^{3} \rightarrow \Lambda \mathbb{S}^{2}$.

Preuve. - L'argument est algébrique et porte sur les classes d'Euler de ces deux fibrés. En effet, les bases de ces deux fibrés sont homotopiquement équivalents d'après le théorème IV.6. Par ailleurs, un $\mathbb{S}^{1}$-fibré $p: E \rightarrow B$ est classifié par sa classe d'Euler $e(E)$ qui est un élément de $H^{2}(B ; \mathbb{Z})$. Il suffit donc de montrer que le pull-back de $e\left(\Lambda \mathbb{S}^{2} \times_{\mathbb{S}^{2}} \mathbb{S}^{3}\right)$ par l'application $c$ coïncide avec $e\left(K \mathbb{S}^{2}\right)$, c'est à dire de prouver l'égalité $e\left(K \mathbb{S}^{2}\right)=c^{*} e\left(\Lambda \mathbb{S}^{2} \times_{\mathbb{S}^{2}} \mathbb{S}^{3}\right)$. Si $E \rightarrow B$ est un fibré en cercle de classe d'Euler $e(E)$, on notera également $e(E): H_{2}(B) \rightarrow \mathbb{Z}$ un représentant de $e(E) \in H^{2}(B ; \mathbb{Z})$, qui consiste en une application de $\mathbb{Z}$-modules. L'évaluation de $e(E)$ sur un cycle $u$, est notée $<e(E), u>$.

Connaissant l'homologie de $\Lambda \mathbb{S}^{2}$, on exhibe un représentant explicite de $e\left(\Lambda \mathbb{S}^{2} \times_{\mathbb{S}^{2}}\right.$ 
$\left.\mathbb{S}^{3}\right)$. La fibration $\Omega \mathbb{S}^{2} \stackrel{i}{\rightarrow} \Lambda \mathbb{S}^{2} \stackrel{e v^{2}}{\rightarrow} \mathbb{S}^{2}$ donne la suite exacte en homologie:

$$
0 \rightarrow H_{2}\left(\Omega \mathbb{S}^{2}\right)=\mathbb{Z} \stackrel{i_{2}}{\rightarrow} H_{2}\left(\Lambda \mathbb{S}^{2}\right) \stackrel{e v_{2}^{2}}{\rightarrow} H_{2}\left(\mathbb{S}^{2}\right)=\mathbb{Z} \rightarrow 0
$$

où $i_{2}(x)=(x, 0)$ et $e v_{2}^{2}(x, y)=y$.

On va montrer successivement les affirmations suivantes:

1. $\operatorname{Ker} e\left(\Lambda \mathbb{S}^{2} \times_{\mathbb{S}^{2}} \mathbb{S}^{3}\right)=H_{2}\left(\Omega \mathbb{S}^{2}\right)$.

2. $\operatorname{Ker} c^{*} e\left(\Lambda \mathbb{S}^{2} \times_{\mathbb{S}^{2}} \mathbb{S}^{3}\right)=i_{2}^{c o m b}\left(H_{2}\left(J \mathbb{S}^{1}\right)\right)$.

3. $\operatorname{Ker} e\left(K \mathbb{S}^{2}\right) \supset i_{2}^{c o m b}\left(H_{2}\left(J \mathbb{S}^{1}\right)\right)$.

4. $c^{*} e\left(\Lambda \mathbb{S}^{2} \times_{\mathbb{S}^{2}} \mathbb{S}^{3}\right)=e\left(K \mathbb{S}^{2}\right)$.

Point 1: Puisque $\Lambda \mathbb{S}^{2} \times_{\mathbb{S}^{2}} \mathbb{S}^{3}$ est le pull-back du fibré de Hopf, on a $e\left(\Lambda \mathbb{S}^{2} \times_{\mathbb{S}^{2}} \mathbb{S}^{3}\right)=$ $e\left(\mathbb{S}^{3}\right) \circ e v_{2}^{2}$ en tant qu'éléments de $H_{0} m_{\mathbb{Z}}\left(H_{2}\left(\Lambda \mathbb{S}^{2}\right), \mathbb{Z}\right)$. De la suite exacte IV.8 On déduit $\operatorname{Ker}\left[e\left(\Lambda \mathbb{S}^{2} \times_{\mathbb{S}^{2}} \mathbb{S}^{3}\right)\right]=H_{2}\left(\Omega \mathbb{S}^{2}\right)$, ce qui montre le point 1 .

Point 2: Le point 1. implique le point 2. de la manière suivante. Considérons le diagramme commutatif suivant:

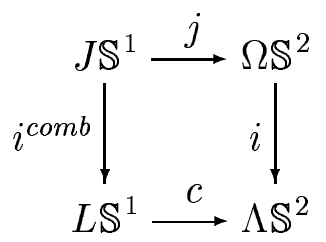

où $i$ est l'inclusion et $i^{c o m b}$ est l'application définie par:

$$
i^{c o m b}(x)=\left(x,\left(\left[u_{k}(x) ; u_{k+1}(x)\right]\right)_{k \in<0 ; n-1>}\right)
$$

où $u_{i}(x)=\frac{\sum_{k=1}^{i} d\left(x_{k} ; *\right)}{\sum_{k=1}^{n} d\left(x_{k} ; *\right)}$. Il donne en homologie de degré 2 l'égalité $i_{2} \circ j_{2}=c_{2} \circ i_{2}^{c o m b}$. Or $c_{2}$ est un isomorphisme de $\mathbb{Z}$-modules. On en déduit que 1 . implique 2.

Point 3: Pour montrer le point 3. considérons le pull-back du fibré $K \mathbb{S}^{2}$ par l'application $i^{\text {comb }}$ :

$$
I \mathbb{S}^{2}=\left\{(u,[x, a, z]) \in J \mathbb{S}^{1} \times K \mathbb{S}^{2} \mid i^{c o m b}(u)=([x],[a])\right\}
$$

Remarquons que l'application $i^{\text {comb }}$ est continue à valeurs dans $\left(\mathbb{S}^{1}\right)^{n} \times \widetilde{F_{0}\left(\mathbb{S}^{1}, n\right)}$, (les intervalles $\left[u_{i}(u) ; u_{i+1}(u)\right]$ forment une partition de $\left.[0 ; 1]\right)$. On note $\tilde{a}(u)$ cette configuration ordonnée d'intervalles. L'application

$$
k:(u,[z]) \in J \mathbb{S}^{1} \times \mathbb{S}^{1} \mapsto(u,[x, \tilde{a}(u), z]) \in I \mathbb{S}^{2}
$$

est alors un morphisme de $\mathbb{S}^{1}$-fibré au dessus de $J \mathbb{S}^{1}$ et admet pour réciproque

$$
l:(u,[x, y, z]) \in I \mathbb{S}^{2} \mapsto(u,[z]) \in J \mathbb{S}^{1} \times \mathbb{S}^{1}
$$


ce qui démontre que $I \mathbb{S}^{2}$ est le fibré trivial sur $J \mathbb{S}^{1}$. On a ainsi $i^{c o m b}\left(e\left(K \mathbb{S}^{2}\right)\right)=$ $e\left(I \mathbb{S}^{2}\right)=0$, ce qui implique que si $x=i_{2}^{c o m b}(y) \in H_{2}\left(J \mathbb{S}^{1}\right)$, alors $e\left(K \mathbb{S}^{2}\right)(x)=e\left(K \mathbb{S}^{2}\right) \circ$ $i_{2}(y)=0$, et ceci démontre le point 3 .

Point 4: Pour le dernier point, on évalue la classe d'Euler de chaque fibré sur le 2-cycle non nul représentant le tore $\mathbb{T}^{2}=\mathbb{S}^{1} \times F_{0}(1) \rightarrow L \mathbb{S}^{1}$ dans $H_{2}\left(L \mathbb{S}^{1}\right)=\mathbb{Z} \oplus \mathbb{Z}$. Notons ce cycle $u=(p, q)$.

- Calcul de $<c^{*} e\left(\Lambda \mathbb{S}^{2} \times_{\mathbb{S}^{2}} \mathbb{S}^{3}\right), u>$ :

L'application composée

$$
L \mathbb{S}^{1} \stackrel{c}{\rightarrow} \Lambda \mathbb{S}^{2} \stackrel{e v^{2}}{\rightarrow} \mathbb{S}^{2}
$$

restreinte à $\mathbb{T}^{2}$ est de degré 1 , puisque elle contracte les cercles $\mathbb{S}^{1} \times\{0\}$ et $\{0\} \times \mathbb{S}^{1}$ sur le pole nord $N$ de la sphère, et envoie le complémentaire de leur union homéomorphiquement sur $\mathbb{S}^{2} \backslash\{N\}$. Elle envoie donc le générateur $u$ de $H_{2}\left(L \mathbb{S}^{1}\right)$ sur le générateur $w$ de $H_{2}\left(\mathbb{S}^{2}\right)$. Par naturalité de la classe d'Euler, le diagramme commutatif de $\mathbb{S}^{1}$-fibrés:

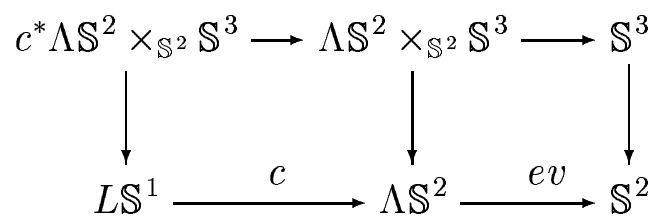

donne l'égalité $<c^{*} e\left(\Lambda \mathbb{S}^{2} \times_{\mathbb{S}^{2}} \mathbb{S}^{3}\right), u>=<e\left(\mathbb{S}^{3}\right), w>=1$ puisque $w$ est générateur de $H_{2}\left(\mathbb{S}^{2}\right)$.

- Calcul de $<e\left(K \mathbb{S}^{2}\right), u>$ :

La restriction du fibré $K \mathbb{S}^{2} \rightarrow L \mathbb{S}^{1}$ au tore $\mathbb{S}^{1} \times F_{0}(1)$ est exactement le fibré de Heisenberg $\mathcal{H}_{1}=H_{3} / Z_{3}$. Sa classe d'Euler évaluée sur le cycle $u$.

Notons $A=c^{*} e\left(\Lambda \mathbb{S}^{2} \times_{\mathbb{S}^{2}} \mathbb{S}^{3}\right)$ et $B=e\left(K \mathbb{S}^{2}\right)$ les applications de $\mathbb{Z}$-modules. Il existe $\left(a_{1}, a_{2}, b_{1}, b_{2}\right) \in \mathbb{Z}^{4}$ tel que:

$$
A(x, y)=a_{1} x+a_{2} y \text { et } B(x, y)=b_{1} x+b_{2} y
$$

Les points 2. et 3 . impliquent successivement $a_{1}=0$ et $b_{1}=0$ puisque $H_{2}\left(J \mathbb{S}^{1}\right)=\mathbb{Z} \oplus 0$. Puisque $\langle A, u\rangle=1=\langle B, u\rangle$, on obtient $a_{2}=b_{2}$ donc $A=B$. On en déduit le point 4. ce qui prouve le théorème.

Proposition IV.2. L'espace $\left(K \mathbb{S}^{2}\right)$. est un $\mathbb{S}^{1}$-fibré principal au dessus de $\left(L \mathbb{S}^{1}\right)$. faiblement homotopiquement équivalent à $\Lambda \mathbb{S}^{2} \times \mathbb{S}^{2} \mathbb{S}^{3}$.

Preuve. - Montrons que l'action du cercle est libre sur $\left(K \mathbb{S}^{2}\right)$. Soit $X \in\left(K \mathbb{S}^{2}\right)$. et $s \in \mathbb{R}$ tel que $X \cdot[s]=X$. Soit $X_{m}$ et $X_{n}$ deux représentants de $X$ dans $\left(E_{m}\right)$. et $\left(E_{n}\right)_{\text {. }}$. Les éléments $X_{m}$ et $X_{n}$ ont nécessairement le même nombre d'éléments $x_{i}$ non nuls. Soit $X_{m}^{k}\left(\operatorname{resp} . X_{n}^{k}\right)$ l'image de $X_{m}$ (resp. $\left.X_{n}\right)$ après quotients successifs par des projections de type $\Pi_{n}^{i}$, où $k$ est le nombre communs de coordonnées $x_{i}$ non-nuls. Notons $X_{m}=\left[x_{1}, \cdots, x_{m}, y_{1}, \cdots, y_{m}, z\right]$ et $X_{n}=\left[x_{1}^{\prime}, \cdots, x_{n}^{\prime}, y_{1}^{\prime}, \cdots, y_{n}^{\prime}, z^{\prime}\right]$. Quitte à permuter cycliquement par $\mathbb{Z} / m \mathbb{Z}$ et $\mathbb{Z} / n \mathbb{Z}$, et à changer $z$ et $z^{\prime}$, on peut supposer que $x_{1}=x_{1}^{\prime}$. Puisque les applications $\Pi_{n}^{i}$ ne change pas la composante en $z$, les éléments 
$X_{m}^{k}$ et $X_{n}^{k}$ ne dépendent pas de l'ordre dans lequel s'effectue les projections. De plus, ces projections sont $\mathbb{S}^{1}$-équivariantes, ce qui implique l'égalité $X_{m}^{k}=X_{n}^{k} \cdot[s]$ dans $\left(E_{k}\right)_{\bullet}$, et entraine (l'action est libre sur chaque $\left.\left(E_{p}\right)_{\bullet}\right)$ que $[s]=0$. L'espace $K \mathbb{S}_{\bullet}^{2}$ est donc un $\mathbb{S}^{1}$-espace principal.

Pour montrer que $K \mathbb{S}_{\text {. }}^{2}$ est un fibré principal, il reste à montrer sa trivialité locale. On filtre $K \mathbb{S}_{\bullet}^{2}\left(\operatorname{resp} . L \mathbb{S}_{\bullet}^{1}\right)$ par les sous-espaces $\left(\left(F_{m}\right)_{\bullet}\right)_{m \geq 0}\left(\operatorname{resp} .\left(\left(D_{m}\right)_{\bullet}\right)_{m \geq 0}\right.$ définies par $\left(F_{m}\right)_{\bullet}=\coprod_{n=0}^{m}\left(E_{n}\right)_{\bullet} / \sim_{m}$ (resp. $\left.\left(D_{m}\right) \bullet=\coprod_{n=0}^{m}\left(B_{n}\right)_{\bullet} / \sim_{m}\right)$, où $\sim_{m}$ est la trace de la relation d'équivalence $\sim$ sur les unions finies respectives. Puis on montre, par récurrence sur $m$, que $\left(F_{m}\right)$ • est un fibré principal de base $\left(D_{m}\right)_{\bullet} . K \mathbb{S}_{\bullet}^{2} \rightarrow L \mathbb{S}_{\bullet}^{1}$ apparait comme la limite inductive des $\mathbb{S}^{1}$-fibrés principaux $\left(F_{m}\right)_{\bullet} \rightarrow\left(D_{m}\right)$. sous les inclusions $\left(F_{m}\right) . \subset\left(F_{m+1}\right)$.. Le passage du rang $n$ au rang $n+1$ se traite de manière analogue à la preuve du lemme IV.2, en utilisant le diagramme commutatif:

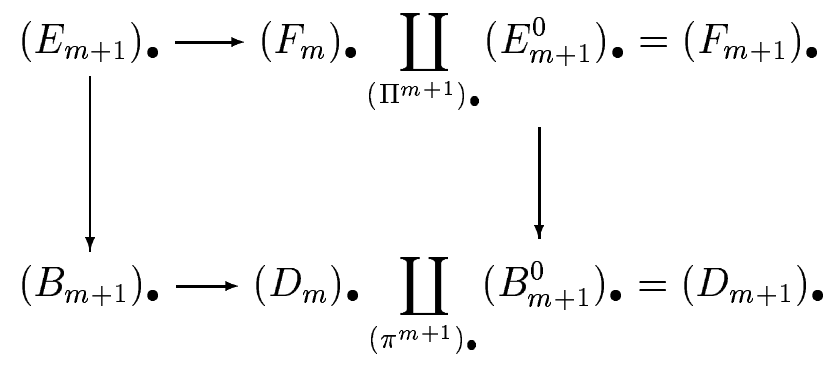

et en remarquant que l'application de recollement $\left(\Pi^{m+1}\right)$. est $\mathbb{S}^{1}$-équivariante et donne au quotient $\pi_{\bullet}^{m+1}$.

De plus, l'application $\widetilde{M i l}$ fournit une équivalence faible d'homotopie entre $K \mathbb{S}^{2}$ et $K \mathbb{S}_{\text {. }}^{2}$ On conclut alors grâce au théorème IV.7.

La démonstration précédente n'est pas complètement détaillée. Remarquons cependant que le corollaire IV.1 va dans le sens du résultat. En effet, celui-ci dit que le pull-back de la flèche $\left(K \mathbb{S}^{2}\right) \bullet \rightarrow\left(L \mathbb{S}^{1}\right) \bullet$ par l'application $M i l$ (voir le diagramme après la proposition IV.1) est un $\mathbb{S}^{1}$-fibré principal.

\section{IV.4.d Le modèle de $\Lambda \mathbb{S}^{3}$}

On commence par une proposition.

Proposition IV.3. $\Pi_{1}\left(K \mathbb{S}^{2}\right)=\Pi_{1}\left(\Lambda \mathbb{S}^{2} \times_{\mathbb{S}^{2}} \mathbb{S}^{3}\right)=\mathbb{Z}$

Preuve. - La première égalité découle du théorème précédent. Pour la deuxième, on construit un quotient de type $\Lambda \mathbb{S}^{3} / \mathbb{Z}$ homéomorphe à $\Lambda \mathbb{S}^{2} \times \mathbb{S}^{2} \mathbb{S}^{3}$.

Considérons l'action naturelle de $\mathbb{Z}$ sur $\Lambda \mathbb{S}^{3}$ suivante. Soit $k \in \mathbb{Z}$, et $f$ un élément de $\Lambda \mathbb{S}^{3}$. On définit alors

$$
k \cdot f: \exp (i x) \in \mathbb{S}^{1} \rightarrow k \cdot f(x)=\exp (i k x) f(\exp (i x)) \in \mathbb{S}^{3}
$$

où $\mathbb{S}^{1}$ est vu comme le cercle unité dans le plan complexe et $\mathbb{S}^{3}$ la sphère unité de $\mathbb{C}^{2}$. 
Cette action est libre et proprement discontinue puisque l'inégalité triangulaire couplée aux égalités $\operatorname{Sup}_{x \in \mathbb{S}^{1}}|1-\exp (i k x)|=2$ pour $k \neq 0$, implique $k \cdot B_{\infty}(f ; 1) \cap$ $B_{\infty}(f ; 1)=\emptyset$. Comme $\Lambda \mathbb{S}^{3}$ est simplement connexe, on obtient $\Pi_{1}\left(\Lambda \mathbb{S}^{3} / \mathbb{Z}\right)=\mathbb{Z}$.

Par ailleurs, l'application $f \in \Lambda \mathbb{S}^{3} \mapsto(h \circ f, f(0)) \in \Lambda \mathbb{S}^{2} \times_{\mathbb{S}^{2}} \mathbb{S}^{3}$ est $\mathbb{S}^{1}$-invariante et donne au qoutient un homéomorphisme entre $\Lambda \mathbb{S}^{3} / \mathbb{Z}$ et $\Lambda \mathbb{S}^{2} \times_{\mathbb{S}^{2}} \mathbb{S}^{3}$ ce qui entraine la seconde égalité.

Rappelons que $x \cdot y$ désigne le produit scalaire euclidien dans $\mathbb{R}^{n}$. Posons

$$
M \mathbb{S}^{2}=\left\{([x, y, z], t) \in K \mathbb{S}^{2} \times \mathbb{R} \mid\left[x \cdot 1_{n}\right]=[t]\right\} \subset K \mathbb{S}^{2} \times \mathbb{R}
$$

et $\left(M \mathbb{S}^{2}\right)$. son analogue défini à partir de $\left(K \mathbb{S}^{2}\right)$. On a alors le corollaire suivant.

Corollaire IV.2. Les espaces $M \mathbb{S}^{2}$ et $\left(M \mathbb{S}^{2}\right)$. sont faiblement homotopiquement équivalents à l'espace $\Lambda \mathbb{S}^{3}$.

Preuve. - Par construction, l'espace $M \mathbb{S}^{2}$ est un revêtement de groupe $\mathbb{Z}$ au dessus de $K \mathbb{S}^{2}$. Mais d'après la proposition IV.3, $\Pi_{1}\left(K \mathbb{S}^{2}\right)$ est aussi $\mathbb{Z}$. On en déduit que $M \mathbb{S}^{2}$ est le revêtement universel de $K \mathbb{S}^{2}$. Le théorème IV.7 implique alors que $M \mathbb{S}^{2}$ est homotopiquement équivalent au revêtement universel de $\Lambda \mathbb{S}^{2} \times_{\mathbb{S}^{2}} \mathbb{S}^{3}$ qui est $\Lambda \mathbb{S}^{3}$.

\section{IV.5 Une application de $B \widetilde{T}$ dans $\Lambda \mathbb{S}^{3}$}

En suivant la stratégie de P.Greenberg décrite au paragraphe IV.2, nous utilisons les modèles de $B \widetilde{T}$ et de $\Lambda \mathbb{S}^{3}$ que sont respectivement $\widehat{\mathcal{G r}} / T$ et $\left(M \mathbb{S}^{2}\right)$. pour construire l'application désirée.

\section{IV.5.a Fonctions de décalage}

Soit $f \in \widehat{\mathcal{G r}}$ et $x \in b k(f) \subset \widehat{\mathbb{Q}}$. Rappelons que $D_{x} f=\left(f_{x}^{r}\right)^{-1} f_{x}^{l}$ est une transformation parabolique (fixant $x$ ) qui mesure le défaut pour $f$ d'être $P S L_{2} \mathbb{R}$ au voisinage de $x$. Puisque $P S L_{2} \mathbb{Z}$ agit transitivement sur $\widehat{\mathbb{Q}}$, il existe $\gamma \in P S L_{2} \mathbb{Z}$ envoyant $x$ sur $\infty$. Le conjugué $\gamma D_{x} f \gamma^{-1}$ est un parabolique en $\infty$. Il est donc de la forme $z \in \mathbb{R} \cup\{\infty\} \mapsto z+d_{x}(f) \in \mathbb{R} \cup\{\infty\}$. La partie de translation $d_{x}(f)$ ne dépend pas de $\gamma \in P S L_{2} \mathbb{Z}$, car les éléments hyperboliques de $P S L_{2} \mathbb{Z}$ ne fixent pas de rationnels.

Définition IV.5.1. (Fonctions de décalage)

Soit $x \in \widehat{\mathbb{Q}}$, la fonction $d_{x}: \widehat{\mathcal{G r}} \rightarrow \mathbb{R}$ est appelée fonction de décalage en $x$. C'est une fonction continue qui mesure quantitativement le défaut d'être $P S L_{2} \mathbb{R}$ au voisinage de $x$.

Le lemme suivant donne une formule de dérivation (déjà formulée dans [Gre4]) pour cette famille de fonctions et la relie aux coordonnées horocycliques.

Lemme IV.4. Pour tout $(f, \gamma, x) \in \widehat{\mathcal{G r}} \times T \times \widehat{\mathbb{Q}}$, on a l'égalité:

$$
d_{x}(f \circ \gamma)=d_{\gamma \cdot x}(f)+d_{x}(\gamma)
$$


De plus, si $f \in \widehat{\mathcal{G} r_{n}}$ et $x \in S_{n}$, on a l'égalité

$$
d_{x}(f)=l_{x}\left(f \cdot b_{n}\right)-l_{x}\left(b_{n}\right)
$$

Enfin la fonction somme:

$$
\sigma: f \in \widehat{\mathcal{G r}} \mapsto \sum_{x \in b k(f)} d_{x}(f) \in \mathbb{R}
$$

est continue et invariante par le groupe $T$.

Preuve. - Par définition, nous avons $D_{x}(f \circ \gamma)=\left(\gamma_{x}^{r}\right)^{-1} D_{\gamma \cdot x}(f) \gamma_{x}^{l}$. En prenant $p_{1} \in P S L_{2} \mathbb{Z}$ tel que $p_{1} \cdot x=\infty$ et $p_{2}=p_{1} \circ\left(\gamma_{x}^{r}\right)^{-1}$, on a les égalités

$$
p_{1} D_{x}(f \circ \gamma) p_{1}^{-1}=t_{d_{x}(f \circ \gamma)} \quad, \quad p_{2} D_{\gamma \cdot x}(f) p_{2}^{-1}=t_{d_{\gamma \cdot x}(f)} \quad \text { et } \quad p_{1} D_{x}(\gamma) p_{1}^{-1}=t_{d_{x}(\gamma)}
$$

où $t_{a}$ désigne la translation $z \mapsto z+a$. En écrivant $D_{x}(f \circ \gamma)=D_{x}(\gamma)\left(\gamma_{x}^{l}\right)^{-1} D_{\gamma \cdot x}(f) \gamma_{x}^{l}$, on obtient par unicité de la conjugaison par $P S L_{2} \mathbb{Z}$,

$$
t_{d_{x}(f \circ \gamma)}=t_{d_{\gamma \cdot x}(f)} \circ t_{d_{x}(\gamma)}
$$

On en déduit ainsi l'égalité IV.9.

Pour la seconde égalité, soit $x \in S_{n}$ et notons $x^{-}$et $x^{+}$les voisins immédiats à gauche et à droite de $x$ parmis les éléments de $S_{n}$. Considérons $P \in P S L_{2} \mathbb{Z}$ et $Q \in P S L_{2} \mathbb{R}$ tels que $P(x)=\infty$ et $Q(f(x))=\infty$. L'élément $Q f P^{-1}$ est un élément de $\widehat{\mathcal{G r}}$ puisque $P \in P S L_{2} \mathbb{Z}$. De sorte que $D_{\infty}\left(Q f P^{-1}\right)$ est bien défini et égal à $P D_{x} f P^{-1}$ puisque $Q \in P S L_{2} \mathbb{R}$. Ainsi, on a $D_{\infty}\left(Q f P^{-1}\right)=t_{d_{x}(f)}$.

De plus, $Q f P^{-1}$ fixe l'infini. Il est donc du type $z \mapsto a^{2} z+d^{+} a$ (resp. $z \mapsto a^{2} z+$ $d^{-} a$ ) sur l'intervalle $\left[\infty ; P\left(x^{+}\right)\right]$(resp. $\left[P\left(x^{-}\right) ; \infty\right]$ ). On calcule alors explicitement que $D_{\infty}\left(Q f P^{-1}\right)=t_{\frac{d^{+}-d^{-}}{a}}$, ce qui entraine $d_{x}(f)=\frac{d^{+}-d^{-}}{a}$.

Calculons les coordonnées horocycliques de $f$ et de $i d$ en $x$. Puisque $P$ est un élément de $P S L_{2} \mathbb{Z}$, on a:

$$
l_{x}\left(b_{n}\right)=l_{\infty}\left(P \cdot b_{n}\right)=\int_{P x^{-}}^{P x^{+}} \frac{d y}{h}=\frac{P\left(x^{+}\right)-P\left(x^{-}\right)}{h}
$$

où $h$ est la hauteur de l'horocycle $P \cdot h_{x}^{F}$ basé en l'infini. De même, on a:

$$
l_{x}\left(f \cdot b_{n}\right)=l_{x}\left(Q f \cdot b_{n}\right)=\int_{Q f\left(x^{+}\right)}^{Q f\left(x^{-}\right)} \frac{d y}{a^{2} h}=\frac{P\left(x^{+}\right)-P\left(x^{-}\right)}{h}+\frac{d^{+}-d^{-}}{a h}
$$

puisque $Q \in P S L_{2} \mathbb{R}$ et que $Q f P^{-1}$ transforme l'horocycle de hauteur $h$ en l'horocycle de hauteur $a^{2} h$.

On obtient donc $l_{x}\left(f \cdot b_{n}\right)-l_{x}\left(b_{n}\right)=\frac{d_{x}(f)}{h}$. Enfin, $P$ étant dans $P S L_{2} \mathbb{Z}$, l'horcocycle $P\left(h_{x}^{F}\right)$ est de Ford. Etant basé en $\infty$, il est à la hauteur 1 sur le demi-plan de Poincaré, ce qui entraine le résultat. 
Concernant la fonction somme, celle-ci est continue pour la topologie faible de $\widehat{\mathcal{G r}}$. Pour l'invariance par $T$, il suffit de remarquer que $\sigma$ est un morphisme de groupes en vertu de la formule IV.9, puis que cette fonction vaut 0 , évaluée sur les deux générateurs $\alpha$ et $\beta$ de $T$, vus en introduction (voir [Loc-Sch] pour une présentation de $T$ ayant pour système de générateurs $\{\alpha ; \beta\}$ et cinq relations).

\section{IV.5.b Construction de $\Phi: \widehat{\mathcal{G} r} \rightarrow\left(K \mathbb{S}^{2}\right)$.}

Soit $f \in \widehat{\mathcal{G} r}$ et $\bar{x}=\left(x_{1}, \cdots, x_{n}\right)$ une indexation de ses points de coupure. Notons $f(\bar{x})$ la configuration ordonnée des images par $f$ de ses points de coupures après application de la fonction $[\tan (\pi \cdot)]^{-1}$ (donnée en IV.1.a). Ainsi, $f(\bar{x})=\left(f\left(x_{1}\right), \cdots, f\left(x_{n}\right)\right)$ est un élément de $F_{0}(n)$. De même, on note en abrégé $d_{\bar{x}}(f)=\left(d_{x_{1}} f, \cdots, d_{x_{n}} f\right) \in \mathbb{R}^{n}$ et $\left[d_{\bar{x}}(f)\right]$ son projeté sur le tore $\mathbb{T}^{n}$.

Notons $\phi(f)=\left[d_{\bar{x}}(f), f(\bar{x})\right] \in\left(B_{n}\right)_{\text {. }}$. Cette définition ne dépend pas du premier point de coupure choisi car $\left(B_{n}\right)$ • est le quotient de $\left(\mathbb{S}^{1}\right)^{n} \times F_{0}(n)$ par $\mathbb{Z} / n \mathbb{Z}$. De plus, l'ajout de faux points de coupure $x^{\prime}$ (dans ce cas $d_{x^{\prime}} f=0$ ) ne change pas la définition de $\phi(f)$ vu comme élément de $\left(L \mathbb{S}^{1}\right)$. On a donc défini une application $\phi: \widehat{\mathcal{G} r} \rightarrow\left(L \mathbb{S}^{1}\right)$.

Lemme IV.5. $\phi$ est continue et invariante par $T$.

Preuve. - Pour la continuité, il suffit de montrer que $\forall n, \phi_{n}=\phi_{\mid \widehat{\mathcal{G r}}{ }_{n}}$ est continue, mais ceci est vrai puisque chaque fonction de coordonnée $f \in \widehat{\mathcal{G} r_{n}} \mapsto d_{x} f \in \mathbb{R}$ et $f \in \widehat{\mathcal{G} r_{n}} \mapsto f(x) \in \mathbb{S}^{1}$ pour $x \in S_{n}$ est continue.

Pour la $T$-invariance, soit $(f, \gamma) \in \widehat{\mathcal{G} r} \times T$. On peut supposer, quitte à ajouter de faux points de coupure, avoir $b k(f \circ \gamma)=\gamma^{-1}(b k(f)) \cup b k(\gamma)=\left(y_{1}, \cdots, y_{k}\right)$. Si $y_{i} \notin \gamma^{-1}(b k(f))$, on a $n_{y_{i}}(f \circ \gamma)=n_{y_{i}}(\gamma) \in \mathbb{Z}$. On peut donc simplifier l'expression de $\phi(f)$, en utilisant la relation d'équivalence sur $\left(L \mathbb{S}^{1}\right)$. jusqu'à obtenir

$$
\phi(f \circ \gamma)=\left[d_{\gamma y_{i_{1}}}(f), \cdot, d_{\gamma y_{i_{s}}}(f), f \circ \gamma\left(y_{i_{1}}\right), \cdots, f \circ \gamma\left(y_{i_{s}}\right)\right]
$$

avec $\gamma^{-1} \cdot b k(f)=\left(y_{i_{1}}, \cdots, y_{i_{s}}\right)$. Après le changement d'indices $y=\gamma^{-1} \cdot x$, on obtient $\phi(f \circ \gamma)=\phi(f)$.

Nous souhaitons relever cette application à $\left(K \mathbb{S}^{2}\right)_{\text {. }}$. Remarquons que l'application $\phi$, à $\operatorname{card}(b k(f))=n$ fixé, transite par l'espace $\mathbb{R}^{n} \times_{\mathbb{Z} / n \mathbb{Z}} F_{0}(n)$ qui a le type d'homotopie du cercle. Un $\mathbb{S}^{1}$-fibré au dessus de cet espace est donc trivial. Par exemple le fibré

$$
E_{n}^{\prime}=\mathbb{Z} / n \mathbb{Z} \backslash\left[\mathbb{R}^{n} \times \widetilde{F_{0}(n)} \times \mathbb{R}\right] / Z_{2}
$$

(où l'action de $Z_{2}$ est donnée par $\left.[x, y, z] *(q, r)=\left[x, y+q, z+r+q \sum_{1}^{n} x_{i}\right]\right)$ admet pour section trivial l'application:

$$
[x, y] \in \mathbb{R}^{n} \times_{\mathbb{Z} / n \mathbb{Z}} F_{0}(n) \mapsto[x, \tilde{y}, x \cdot \tilde{y}] \in E_{n}^{\prime}
$$

où $\tilde{y}$ est un relevé de $y$ et où $x \cdot \tilde{y}$ désigne le produit scalaire entre $x$ et $\tilde{y}$. 
On note $\widetilde{f(\bar{x})} \in \widetilde{F_{0}(n)}$ un relevé à priori quelconque de $f(\bar{x})$. A l'aide de la remarque précédente, on définit l'application suivante:

$$
\begin{array}{rlc}
\Phi: \widehat{\mathcal{G} r} & \rightarrow & \left(K \mathbb{S}^{2}\right) \\
f & \mapsto \quad\left[d_{\bar{x}}(f), \widetilde{f(\bar{x})}, d_{\bar{x}}(f) \cdot \widetilde{f(\bar{x})}\right]
\end{array}
$$

Cette application ne dépend pas du relevé choisi, ni même de l'ordre d'indexation les points de coupure de $f$. Pour le voir, il suffit de remplacer dans l'expression de $\Phi(f)$ le terme $\widetilde{f(\bar{x})} \operatorname{par} \gamma \cdot \widetilde{f(\bar{x})}$ où $\gamma$ agit sur $\widetilde{F_{0}(n)}$ comme suit:

$$
\gamma \cdot\left(y_{1}, \cdots, y_{n}\right)=\left(y_{2}, \cdots, y_{n}, y_{1}+1\right)
$$

On peut également ajouter des faux points de coupure, ce qui n'altére pas le produit scalaire et est donc compatible avec les projections $\left(\Pi_{i}^{n}\right)_{\bullet}$. Rappelons que l'action du cercle relative au $\mathbb{S}^{1}$-fibré $\left(K \mathbb{S}^{2}\right)$. est donnée par la formule $[x, y, z] \cdot[s]=[x, y, z+s]$.

Proposition IV.4. L'application $\Phi$ est un relevé continu de $\phi$ et vérifie la formule suivante:

$$
\Phi(f \circ \gamma)=\Phi(f) \cdot \mu(\gamma)(f)
$$

où

$$
\mu(\gamma)(f)=\sum_{y \in b k(\gamma)} d_{y}(\gamma) f(\gamma y) \in \mathbb{S}^{1}
$$

Preuve. - Remarquons d'abord que l'expression de $\mu(\gamma)(f)$ est bien définie sur le cercle $\mathbb{S}^{1}=\mathbb{R} / \mathbb{Z}$ car les nombre $d_{x}(\gamma)$ sont des entiers. La continuité de $\Phi$ se traite de même manière que celle de $\phi$, en notant que, puisque l'application ne dépend pas des relevés choisis, on peut prendre ceux-ci de sorte qu'ils dépendent continument de $f$ localement. L'égalité $p_{\bullet} \circ \phi=\Phi$ est immédiate.

Il reste à montrer la formule IV.11. Quitte à ajouter des points de coupure, on peut supposer que $\Phi(f \circ \gamma)=\left[d_{\bar{z}}(f \circ \gamma), \widehat{f \circ \gamma(\bar{z})}, d_{\bar{z}}(f \circ \gamma) \cdot \widetilde{f \circ \gamma(\bar{z})}\right]$ où $b k(\gamma) \cup \gamma^{-1} b k(f)=$ $\left(z_{1}, \cdots, z_{p}\right)=\bar{z}$. D'après la formule IV.9, on a

$$
d_{\bar{z}}(f \circ \gamma) \cdot \widetilde{f \circ \gamma(\bar{z})}=\sum_{z \in \gamma^{-1} \cdot b k(f)} d_{\gamma \cdot z}(f) \widehat{f \circ \gamma(z)}+\sum_{z \in b k(\gamma)} d_{z}(\gamma) \widehat{f \circ \gamma(z)}
$$

Notons $\widetilde{f\left(x_{1}\right)}<\widetilde{f\left(x_{2}\right)}<\cdots<\widetilde{f\left(x_{n}\right)}$ un relevé de $f(\bar{x})$ où $\bar{x}$ est une configuration ordonnée des points de coupure de $f$, et $\widetilde{f \circ \gamma}\left(y_{1}\right)<\cdots<\widetilde{f \circ \gamma}\left(y_{n}\right)$ un relevé de $(f \circ \gamma)(\bar{y})$ où $\bar{y}$ est une configuration ordonnée de l'ensemble $\gamma^{-1} b k(f)$. Puisque $\gamma$ présèrve l'orientation, il existe un entier $k$ tel que $\gamma y_{i}=x_{k+i}$ pour tout $i \in \mathbb{Z} / n \mathbb{Z}$. On obtient alors:

$$
\widetilde{f \circ \gamma\left(y_{i}\right)}=\left\{\begin{array}{ccc}
\widetilde{f\left(x_{i+k}\right)} & \text { si } & 1 \leq i<n-k \\
f\left(x_{i+k}\right)+1 & \text { si } & n-k+1 \leq i \leq n
\end{array}\right.
$$


Revenons à $\Phi$. On peut supprimer, dans l'expression de $\Phi(f \circ \gamma)$, les termes relatifs aux points n'appartenant pas à $\gamma^{-1} b k(f)$, car en ces points, le décalage de $f \circ \gamma$ est un entier. On obtient donc, après projections successives par les $\Pi_{i}^{n}$, l'expression suivante:

$$
\Phi(f \circ \gamma)=\left[\begin{array}{c}
d_{\gamma y_{1}}(f), \cdots, \widehat{d_{\gamma y_{n}}(f)}, \widehat{f \circ \gamma\left(y_{1}\right)}, \cdots, \widetilde{f \circ \gamma\left(y_{n}\right)}, \\
\sum_{1}^{n} d_{\gamma y_{i}}(f) \widetilde{f \circ \gamma\left(y_{i}\right)}+\sum_{z \in b k(\gamma)} d_{z}(\gamma) \widetilde{f \circ \gamma(z)}
\end{array}\right]
$$

En remplaçant $\widetilde{f \circ \gamma\left(y_{i}\right)}$ par son expression en fonction de $\widetilde{f\left(x_{i+k}\right)}$, on obtient:

$$
\Phi(f \circ \gamma)=\left[\begin{array}{c}
d_{x_{k+1}} f, \cdots, d_{x_{k}} f, \widetilde{f\left(x_{k+1}\right)}, \cdots, \widetilde{f\left(x_{n}\right)}, \widetilde{f\left(x_{k}\right)}+1, \widetilde{f\left(x_{k}\right)}+1, \\
\sum_{1}^{n} d_{x_{i}}(f) \widetilde{f\left(x_{i}\right)}+\sum_{i=1}^{k} d_{x_{i}}(f)
\end{array}\right] \cdot[\mu(\gamma)(f)]
$$

Mais le premier terme du membre de droite est exactement $\gamma_{n}^{k} \cdot \Phi(f)$, où $\gamma_{n}$ est le générateur de l'action de $\mathbb{Z} / n \mathbb{Z}$ sur $\left(\mathcal{K}_{n}\right)_{\text {. }}$. On en déduit la proposition.

Le défaut d'invariance de l'application $\Phi$, mesuré par $\mu(\gamma)(f)$ s'interprète très naturellement dans le problème de classification des fibrés en cercles sur $\widehat{\mathcal{G r}} / T$. Nous rappelons maintenant le contexte général de ce problème.

\section{IV.5.c Nature cohomologique du problème de relèvement de $\phi$}

Soit $p: \widetilde{X} \rightarrow X$ un revêtement de groupe $T$ agissant à droite sur $\widetilde{X}$. L'espace des applications continues de $\widetilde{X}$ dans $\mathbb{S}^{1}$ (appelons le $M$ ) est un groupe abélien muni d'une action à gauche de $T$ définie par:

$$
\gamma \cdot s: f \in \widetilde{X} \mapsto s(f \cdot \gamma) \in \mathbb{S}^{1} \quad \forall(f, \gamma) \in \widehat{\mathcal{G} r} \times T
$$

Il s'agit en particulier d'un $T$-module à gauche. On peut alors définir la cohomologie du groupe $T$ à coéfficients dans $M$. Nous prendrons le point de vue des cochaines nonhomogènes. Par exemple, l'espace des 1-cochaines est $C^{1}(T ; M)=\operatorname{Map}(T ; M)$. Un 1-cocycle est une 1-cochaine vérifiant $\mu\left(\gamma_{1} \gamma_{2}\right)=\gamma_{1} \cdot \mu\left(\gamma_{2}\right)+\mu\left(\gamma_{1}\right)$. Un 1-cobord est une 1-cochaine admettant une primitive, c'est à dire une 0-cochaine $s \in C^{0}(T ; M)=M$ telle que $\gamma \cdot s-s=\mu(\gamma)$. Un cobord est nécessairement un cocycle. Le premier groupe de cohomologie $H^{1}(T ; M)$ est l'ensemble des cocycles quotienté par les cobords.

Théorème IV.8. Il existe une correspondance biunivoque entre $H^{1}(T ; M)$ et l'ensemble des $\mathbb{S}^{1}$-fibrés sur $X$ dont le pull-back sur $\widetilde{X}$ est trivial.

Une manière de construire un tel fibré à partir d'un cocycle $\mu$ est de suspendre le fibré trivial $\widetilde{X} \times \mathbb{S}^{1}$. Plus précisément, le groupe $T$ agit sur ce produit. L'action est donné par:

$$
\gamma \cdot(x, s) \in T \times\left(\widetilde{X} \times \mathbb{S}^{1}\right) \mapsto(x \cdot \gamma, \mu(\gamma)(x)+s) \in \widetilde{X} \times \mathbb{S}^{1}
$$


Le quotient $\widetilde{X} \times_{T} \mathbb{S}^{1}$ est un fibré en cercle sur $X$ dont le pull-back par l'application de revêtement n'est autre que le produit $\widetilde{X} \times \mathbb{S}^{1}$.

Réciproquement, si l'on se donne un tel fibré $E \rightarrow X$. Soit $s: \widetilde{X} \rightarrow p^{*}(E)$ une section du pull-back de $E$ (trivial par hypothèse). L'application $s$ n'est pas invariante par $T$ si $E$ n'est pas trivial. Soit $(\gamma, x) \in T \times \tilde{X}$. Puisque $x \cdot \gamma$ et $x$ ont même image par $p$, leurs images par $s$ sont sur la même fibre. Notons $\mu(\gamma)(x)=s(x \cdot \gamma)-s(x)$. Cela définit un cocycle $\mu$ dans $H^{1}(T ; M)$, qui ne dépend pas de la section $s$. On peut montrer que ces deux constructions sont inverses l'une de l'autre.

Revenons à notre problème initial. Il s'agit d'un cas concret du problème précédent pour $\widehat{\mathcal{G} r}=\widetilde{X}$ et $\widehat{\mathcal{G r}} / T=X$. L'application $\Phi$ est une section du fibré pull-back $\phi^{*}\left(\left(K \mathbb{S}^{2}\right) \bullet\right)$. La méthode précédente (cas général), pour construire le cocycle $\mu$ correspond exactement à la façon dont nous avons calculé le défaut d'invariance de $\Phi$.

Lemme IV.6. L'application $\mu$ est un 1-cocycle.

Preuve. - Il suffit de montrer que $d \mu=0$. Soit $\left(\gamma_{1}, \gamma_{2}, f\right) \in T^{2} \times \widehat{\mathcal{G r}}$. Par définition de $\mu$, on a $\mu\left(\gamma_{1}, \gamma_{2}\right)(f)=\sum_{x \in \widehat{\mathbb{Q}}} d_{x}\left(\gamma_{1} \gamma_{2}\right) f \circ \gamma_{1} \gamma_{2}(x)$. En utilisant l'égalité IV.9 appliquée à $\gamma_{1} \circ \gamma_{2}$, on obtient:

$$
\mu\left(\gamma_{1}, \gamma_{2}\right)(f)=\sum_{x \in \widehat{\mathbb{Q}}} d_{\gamma_{2} \cdot x}\left(\gamma_{1}\right) f \circ \gamma_{1}\left(\gamma_{2} \cdot x\right)+\sum_{x \in \widehat{\mathbb{Q}}} d_{x}\left(\gamma_{2}\right)\left(f \circ \gamma_{1}\right) \circ \gamma_{2}(x)
$$

Le premier membre de droite est égal à $\mu\left(\gamma_{1}\right)(f)$ après avoir effectué le changement de variable $y=\gamma_{2} \cdot x$. Le second est exactement $\mu\left(\gamma_{2}\right)$ évalué en $f \circ \gamma_{1}$, ce qui démontre que $\mu\left(\gamma_{1} \gamma_{2}\right)(f)=\gamma_{1} \cdot \mu\left(\gamma_{2}\right)(f)+\mu\left(\gamma_{1}\right)(f)$. On a ainsi $d \mu=0$.

Nous allons montrer dans la section IV.6.b que ce cocycle est nul. Soit $\theta$ une primitive de $\mu$, c'est à dire un élément du $T$-module $M$ tel que:

$$
\mu(\gamma)(f)=s(f \circ \gamma)-s(f) \quad \forall(f, \gamma) \in \widehat{\mathcal{G} r} \times T
$$

Considérons l'application modifiée $\Phi_{\theta}=\Phi \cdot \theta: \widehat{\mathcal{G r}} \rightarrow\left(K \mathbb{S}^{2}\right)$. définie par:

$$
\Phi_{\theta}(f)=\left[d_{\bar{x}}(f), \widetilde{f(\bar{x})}, d_{\bar{x}}(f) \cdot \widetilde{f(\bar{x})}+\theta(f)\right]
$$

La formule IV.11 dans la proposition IV.4 entraine que pour toute primitive $\theta$, l'application $\Phi_{\theta}$ est invariante par $T$.

On a ainsi, par passage au quotient, définie une application continue de $\widehat{\mathcal{G r}} / T$ à valeurs dans le modèle (des points) de $\Lambda \mathbb{S}^{2} \times_{\mathbb{S}^{2}} \mathbb{S}^{3}$.

\section{IV.5.d Procédé de construction de Borel}

Si $\Gamma$ est un groupe topologique opérant continuement sur un espace topologique $X$, on peut convertir son action en une action libre sur un espace $X^{\prime}$ homotopiquement équivalent à $X$. Considérons le $\Gamma$-fibré universel $E \Gamma \rightarrow B \Gamma$ (défini à homotopie près). 
C'est à dire que tout $\Gamma$-fibré s'obtient comme pull-back de ce fibré. Le groupe $\Gamma$ agit librement sur $E \Gamma$ pour donner au quotient $B \Gamma=E \Gamma / \Gamma$, le classifiant homotopique du groupe $\Gamma$. Soit $X^{\prime}=X \times E \Gamma$ sur lequel $\Gamma$ agit diagonalement. L'action de $\Gamma$ sur le second terme assure que $\Gamma$ agit librement sur ce produit, et puisque $E \Gamma$ est contractile, $X^{\prime}$ est homotopiquement équivalent à $X$. Ce procédé s'appelle procédé de construction de Borel et permet donc de contourner dans certaines situations les problèmes de symétries apparaissant lors de l'étude d'actions de groupes.

\section{IV.5.e La $\mathbb{S}^{1}$-équivariance de $\psi$}

La fonction somme $\sigma$ (lemme IV.4), permet de relever l'application $\Phi$ à $\left(M \mathbb{S}^{2}\right)$. par l'application continue:

$$
\begin{aligned}
\psi: \widehat{\mathcal{G} r} & \rightarrow\left(M \mathbb{S}^{2}\right) . \\
f & \mapsto(\Phi), \sigma(f))
\end{aligned}
$$

Pour calculer l'homologie de $\widetilde{T}$ puis celle de $T$ à partir de celle de $\widetilde{T}$, il faut quotienter $B \widetilde{T}$ par une action de $\mathbb{S}^{1}$. Celle-ci n'étant pas libre, on applique la construction de Borel.

Le cercle $\mathbb{S}^{1}=P S O_{2}(\mathbb{R}) \subset P S L_{2} \mathbb{R}$ agit sur $\widehat{\mathcal{G r}}$ par composition à gauche. De plus, l'application $\tan (\pi \cdot): x \in \mathbb{R} / \mathbb{Z} \rightarrow \tan (\pi x) \in \mathbb{R} \cup\{\infty\}$ est $\mathbb{S}^{1}$-équivariante pour les actions du cercle par translation $t \mapsto t+s$ sur $\mathbb{R} / \mathbb{Z}$ et par $P S O_{2}(\mathbb{R})$ sur $\mathbb{R} \cup\{\infty\}$. L'action passe au quotient $\widehat{\mathcal{G r}} / T$ :

$$
\begin{array}{lcccc}
\mathbb{S}^{1} & \times & \widehat{\mathcal{G}} r & \rightarrow T & \widehat{\mathcal{G} r} / T \\
([s] & , & f) & \mapsto & {[s] \cdot f=\rho_{[s]} \circ f}
\end{array}
$$

où

$$
\rho_{[s]}=\left[\begin{array}{cc}
\cos (\pi s) & -\sin (\pi s) \\
\sin (\pi s) & \cos (\pi s)
\end{array}\right] \in P S O_{2}(\mathbb{R})
$$

Puisque $\left(M \mathbb{S}^{2}\right)$. est homotopiquement équivalent à l'espace des lacets de la sphère $\mathbb{S}^{3}$, il possède l'action naturelle du cercle suivante:

$$
\begin{array}{ccccc}
\mathbb{S}^{1} & \times & \left(M \mathbb{S}^{2}\right) \cdot & \rightarrow & \left(M \mathbb{S}^{2}\right) \\
([s] & , & ([x, y, z], u) & \mapsto & ([x, y+s, z+s u], u)
\end{array}
$$

Celle-ci est bien définie. En effet, si $s^{\prime}-s=k \in \mathbb{Z}$ et $([x, y, z], u) \in\left(M \mathbb{S}^{2}\right)$, on a

$$
\left[s^{\prime}\right] \cdot([x, y, z], u)=([x, y+s, z+s u] *(0, k, 0), u)=[s] \cdot([x, y, z], u)
$$

car $u=\sum_{1}^{n} x_{i}$ et cette dernière condition est préservée par l'action de $\mathbb{S}^{1}$.

Lemme IV.7. L'application $\psi: \widehat{\mathcal{G} r} \rightarrow\left(M \mathbb{S}^{2}\right)$. est $\mathbb{S}^{1}$-équivariante. 
Preuve. - Celle-ci provient directement de la $\mathbb{S}^{1}$-équivariance de la fonction $\tan (\pi \cdot)$ couplée à l'invariance des fonctions de décalage pour l'action de $P S L_{2} \mathbb{R}$ sur $\widehat{\mathcal{G r}}$ par composition à gauche.

Concernant la version modifiée de $\psi$, on admet l'hypothèse suivante sur la primitive $\theta$ du cocycle $\mu$. On donnera une preuve de la nullité de $[\mu]$ à la fin de IV.6.b (corollaire IV.5).

- $\left(\mathbb{S}^{1}\right.$-INV): "Il existe une primitive $\theta$ de $\mu$, invariante pour l'action du cercle".

Corollaire IV.3. L'application $\psi_{\theta}=\left(\Phi_{\theta}, \sigma\right)$ est $\mathbb{S}^{1}$-équivariante et invariante par $T$.

Preuve. - L'invariance de $\theta$ par $\mathbb{S}^{1}$ couplée à la $\mathbb{S}^{1}$-équivariance de $\psi$ entraine celle de $\psi_{\theta}$. L'invariance par $T$ provient de celles de $\Phi \cdot \theta$ et de $\sigma: \widehat{\mathcal{G} r} \rightarrow \mathbb{R}$.

Muni de l'application $\mathbb{S}^{1}$-équivariante $\psi_{\theta}: \widehat{\mathcal{G r}} / T \rightarrow \Lambda \mathbb{S}^{3}$, on définit l'application continue suivante:

$$
\begin{aligned}
& \psi_{\theta} \times_{\mathbb{S}^{1}} i d: \widehat{\mathcal{G} r} / T \times_{\mathbb{S}^{1}} E \mathbb{S}^{1} \rightarrow \quad \mathcal{L S}^{3} \\
& (f, x) \quad \mapsto \quad\left(\psi_{\theta}(f), x\right)
\end{aligned}
$$

Dans la suite du texte, on omettra l'indice $\theta$ dans $\psi_{\theta}$.

\section{IV.6 L'isomorphisme en homologie entière}

Cette section est entièrement consacré à la démonstration du:

Théorème IV.9. L'application $\psi$ donne un isomorphisme en homologie entière.

\section{IV.6.a Stratégie: la connaissance des structures de $H_{*}$}

L'idée de démonstration est la suivante. Puisque nous savons déjà que les homologies du groupe $\widetilde{T}$ et de l'espace des lacets $\Lambda \mathbb{S}^{3}$ sont isomorphes, il suffit de trouver des générateurs libres de $H_{*}(B \widetilde{T})$, dont les images par $\psi_{*}$ sont les générateurs libres de $H_{*}\left(\Lambda \mathbb{S}^{3}\right)$. A première vue, cette stratégie semble quelque peu insatisfaisante puisque elle utilise la connaissance d'un tel isomorphisme. Cependant, le but de cette partie n'est pas tant une nouvelle démonstration du théorème IV.1, qu'une interprétation géométrique, via les outils de la géométrie $\mathrm{CPP}$, du résultat. Rappelons la structure de l'homologie de $\Lambda \mathbb{S}^{3}$. Puisque $\Lambda \mathbb{S}^{3}$ est homéomorphe au produit $\Omega \mathbb{S}^{3} \times \mathbb{S}^{3}$, (la sphère $\mathbb{S}^{3}$ est muni d'une structure de groupe) la formule de Kunneth donne:

$$
H_{*}\left(\Lambda \mathbb{S}^{3}\right)=H_{*}\left(\Omega \mathbb{S}^{3}\right) \otimes H_{*}\left(\mathbb{S}^{3}\right)
$$


Puisque $\Omega \mathbb{S}^{3}$ est muni d'une opération produit continue, la composition des chemins (on dit qu'il s'agit d'un H-espace), son homologie bénéficie d'une structure algébrique supplémentaire, le produit de Pontryagin. Il s'agit de la composition des applications

$$
H_{*}\left(\Omega \mathbb{S}^{3}\right) \otimes H_{*}\left(\Omega \mathbb{S}^{3}\right) \stackrel{\times}{\rightarrow} H_{*}\left(\Omega \mathbb{S}^{3} \times \Omega \mathbb{S}^{3}\right) \stackrel{\circ}{\rightarrow} H_{*}\left(\Omega \mathbb{S}^{3}\right)
$$

où $\times$ désigne le produit croisé en homologie et $\circ$ la composition des chemins dans l'espace $\Omega \mathbb{S}^{3}$.

Ainsi, via le modèle de James $J \mathbb{S}^{2}$ dont la $\mathrm{H}$-structure est définie par la concaténation des mots, on obtient que $H_{*}\left(\Omega \mathbb{S}^{3}\right)=\mathbb{Z}[u]$ est l'anneau des polynomes à coefficients dans $\mathbb{Z}$, ayant pour générateur, l'image du générateur de $H_{2}\left(\mathbb{S}^{2}\right)$ par l'application $\mathbb{S}^{2} \rightarrow J \mathbb{S}^{2}$. Finalement, on obtient les groupes d'homologie de $\Lambda \mathbb{S}^{3}$ (et ceux de $\widetilde{T})$ :

$$
H_{n}\left(\Lambda \mathbb{S}^{3} ; \mathbb{Z}\right)=\mathbb{Z}=H_{n}(B \widetilde{T} ; \mathbb{Z}) \quad \forall n \geq 1
$$

Nous adoptons la démarche suivante.

En IV.6.b, on construit un tore $\mathbb{T}^{2}$ dans l'espace $\widehat{\mathcal{G r}} / T$, dont la classe en homologie $\sigma$ est le générateur de $H_{2}(\widehat{\mathcal{G r}} / T)$.

En IV.6.c, on montre que $\psi_{2}(\sigma)$ est le générateur de $H_{2}\left(\left(M \mathbb{S}^{2}\right) \bullet\right)$, en utilisant le produit de Pontryagin.

En IV.6.d, on prouve, par un procédé de saturation par la fibre, pour les $\mathbb{S}^{1}$ fibrations (obtenues par le procédé de Borel) associées aux espaces $\widehat{\mathcal{G} r} / T$ et $\left(M \mathbb{S}^{2}\right)$, que $\psi$ envoie le générateur de $H_{3}(\widehat{\mathcal{G r}} / T)$ sur celui de $H_{3}\left(\left(M \mathbb{S}^{2}\right)_{\bullet}\right)$.

En IV.6.e, on montre pour tout $n$ que $\psi$ envoie le générateur de $H_{2 n}(B \widetilde{T} ; \mathbb{Z}$ ) (resp. $\left.H_{2 n+1}(B \widetilde{T} ; \mathbb{Z})\right)$ sur celui de $H_{2 n}\left(\Lambda \mathbb{S}^{3} ; \mathbb{Z}\right)$ (resp. $\left.H_{2 n+1}\left(\Lambda \mathbb{S}^{3} ; \mathbb{Z}\right)\right)$, ce qui conclut la démonstration.

\section{IV.6.b Le générateur de $H_{2}(B \widetilde{T})$}

Rappelons que l'espace quotient $\widehat{\mathcal{G} r} / T$ est un classifiant de $\widetilde{T}$ (corollaire III.2). Nous construisons maintenant un plongement du tore $\mathbb{T}^{2}$ dans $\widehat{\mathcal{G r}} / T$ dont le cycle repésentant $u$ est un générateur de $H_{2}(\widehat{\mathcal{G} r} / T)$.

Nous utilisons ici les normalisations pour $\mathcal{G} r$ et $F^{\prime}$ suivantes:

$$
\mathcal{G} r=\left\{f \in \mathcal{G} r \mid f_{0}^{r}=i d\right\} \text { et } F^{\prime}=\left\{\gamma \in T \mid \gamma_{0}^{l}=\gamma_{0}^{r}=i d\right\}
$$

Notons que l'action de $F^{\prime} \subset T$ par composition à droite sur $\widehat{\mathcal{G r}}$ fixe le nouveau normalisé $\mathcal{G} r$. On obtient ainsi l'application $\rho: \mathcal{G} r / F^{\prime} \rightarrow \widehat{\mathcal{G} r} / T$ qui induit au niveau des groupes fondamentaux l'inclusion $F^{\prime} \subset \widetilde{T}$.

Soit $I=[1 ; 2]$. Considérons les arêtes de Farey suivantes:

$$
e_{0}=(\infty ;-2) \quad e_{0}^{\prime}=(2 ; \infty) \quad e_{1}=(-2 ;-1) \quad e_{1}^{\prime}=(1 ; 2)
$$


Pour $s \in I$, on note $g_{s}=h_{s}^{-1} \circ \theta_{s, \tau_{*}, e_{1}}$, la déformation log-lambda modifiée le long de l'arête $e_{1}$. C'est à dire que $h_{t}$ est le morceau hyperbolique de $\theta_{s, \tau_{*}, e_{1}}$ sur l'intervalle $[-1 ; \infty]$. On note ensuite pour $(s, t) \in I^{2}, g_{s, t}^{\prime}=h_{s, t}^{\prime} \circ \theta_{t, g_{s} \cdot \tau_{*}, g_{s} \cdot e_{1}^{\prime}}$ la déformation log-lambda modifiée le long de $g_{s} \cdot e_{1}^{\prime}$ par rapport à la tesselation $g_{s} \cdot \tau_{*}$. L'élément hyperbolique $h_{s, t}^{\prime}$ correspond à la restriction de $\theta_{t, g_{s} \cdot \tau_{*}, g_{s} \cdot e_{1}^{\prime}}$ sur l'intervalle $\left[g_{s}(\infty) ; g_{s}(1)\right]=[\infty ; 1]$.

Notons $\tilde{\mu}(s, t)$ l'élément composé $g_{s, t}^{\prime} \circ g_{s}$. Il s'agit d'un élément de classe CPP. L'ensemble des points de coupure de $\tilde{\mu}$ est contenu dans $\left\{1 ; \frac{3}{2} ; 2 ; \infty ;-2 ;-\frac{3}{2} ;-1\right\}$ d'après le lemme III.2. C'est donc un élément de $\widehat{\mathcal{G} r}$.

D'autre part, notons $g$ (resp. $g^{\prime}$ ) l'élément de $T$ correspondant, via le théorème I.2, au mouvement élémentaire le long de l'arête $e_{0}$ (resp. $e_{0}^{\prime}$ ). Il s'agit d'un élément constitué de quatre morceaux et dont les points de coupure sont $-1,-\frac{3}{2},-2$ et $\infty$ (resp. $\infty, 2, \frac{3}{2}$ et 1 ). Par exemple, l'élément $g$ envoie le quadruplet $\left(\infty,-2,-\frac{3}{2},-1\right)$ $\operatorname{sur}(\infty,-3,-2,-1)$.

Lemme IV.8. Dans l'espace $\widehat{\mathcal{G} r}$, on a les égalités suivantes:

$$
\tilde{\mu}(s, 2)=\tilde{\mu}(s, 1) \circ g^{\prime} \quad \tilde{\mu}(2, t)=\tilde{\mu}(1, t) \circ g \quad \forall(s, t) \in I^{2}
$$

Preuve. - Nous montrons les deux faits suivants. Ces applications sont normalisées au sens défini précédemment et leurs coordonnées log-lambda portées par la tesselation de Farey coïncident. On en déduit alors les égalités dans $\widehat{\mathcal{G} r}$.

Pour tout homéomorphisme du cercle, on note $\operatorname{Supp}(f)=\left\{x \in \mathbb{S}^{1} \mid f(x) \neq x\right\}$ son support. Par construction, les supports de $\tilde{\mu}(s, t), g$ et $g^{\prime}$ sont:

$$
\operatorname{Supp}(\tilde{\mu}(s, t))=[-1 ; 1] \quad \operatorname{Supp}(g)=[-1 ; \infty] \quad \operatorname{Supp}\left(g^{\prime}\right)=[\infty ; 1]
$$

Par conséquent, pour tout $(s, t) \in I^{2}$, l'applications $\tilde{\mu}(s, t)$ est normalisée. De même, $g$ et $g^{\prime}$ appartiennent à $F^{\prime}$. Ainsi, les composées $\tilde{\mu}(s, t) \circ g$ et $\tilde{\mu}(s, t) \circ g^{\prime}$ sont également normalisées. Il reste à cacluler leurs coordonnées log-lambda.

Soit $e \in \tau_{*}$. Par définition, on a $\Lambda(\tilde{\mu}(s, t))(e)=\Lambda\left(\left(g_{s, t}^{\prime} \circ g_{s}\right) \cdot b^{F} ;\left(g_{s, t}^{\prime} \circ g_{s}\right) \cdot e\right)$. Grâce au lemme II.1, appliqué à la déformation le long de $g_{s} \cdot e_{1}^{\prime}$, on obtient:

$$
\begin{aligned}
& \Lambda\left(\left(g_{s, t}^{\prime} \circ g_{s}\right) \cdot b^{F} ;\left(g_{s, t}^{\prime} \circ g_{s}\right) \cdot e\right)=\Lambda\left(g_{s} \cdot b^{F} ; g_{s}(e)\right) \text { si } g_{s}(e) \neq g_{s}\left(e^{\prime} 1\right) \\
& \Lambda\left(\left(g_{s, t}^{\prime} \circ g_{s}\right) \cdot b^{F} ;\left(g_{s, t}^{\prime} \circ g_{s}\right) \cdot e\right)=t \Lambda\left(g_{s} \cdot b^{F} ; g_{s}(e)\right) \text { si } g_{s}(e)=g_{s}\left(e^{\prime} 1\right)
\end{aligned}
$$

Puis en l'appliquant à la déformation $g_{s}$ le long de $e_{0}$, on obtient:

$$
\begin{gathered}
\Lambda\left(g_{s} \cdot b^{F} ; g_{s}(e)\right)=\Lambda\left(b^{F} ; e\right) \text { si } e \neq e_{1} \\
\Lambda\left(g_{s} \cdot b^{F} ; g_{s}(e)\right)=s \Lambda\left(b^{F} ; e\right) \text { si } e=e_{1}
\end{gathered}
$$

Puisque $e_{1} \neq e_{1}^{\prime}$, et que $\Lambda\left(b^{F} ; e\right)=\sqrt{2}$ pour tout $e \in \tau_{*}$, on en déduit les coordonnées log-lambda:

$$
\Lambda(\tilde{\mu}(s, t))\left(e_{1}\right)=s \sqrt{2} \quad \Lambda(\tilde{\mu}(s, t))\left(e_{1}^{\prime}\right)=t \sqrt{2} \quad \text { et } \Lambda(\tilde{\mu}(s, t))(e)=\sqrt{2} \forall e \neq e_{1}, e_{1}^{\prime}
$$


Passons à la composée $\tilde{\mu}(s, t) \circ g^{\prime}$. D'après la proposition III.1, si $e$ est une arête de Farey, on a $\Lambda(\tilde{\mu}(s, t) \circ g)(e)=\Lambda(\tilde{\mu}(s, t) ; g(e))$. Pour le calcul de ce dernier terme, il faut distinguer les cas selon que $g(e)$ est (ou n'est pas) une arête de Farey. Or, par construction de $g, e_{1}^{\prime}$ est la seule arête de Farey dont l'image par $g$ n'est pas une arête de Farey.

Pour calculer la lambda-longueur le long de cette arête, nous appliquons la formule de Ptolémée (proposition III.2) pour le quadrilatère de sommets 1,2,3, $\infty$. Par définition de $\tilde{\mu}(s, t)$, le bracelet $\tilde{\mu}(s, t) \cdot b^{F}$ a les lambda- longueurs suivantes:

$$
\begin{gathered}
\Lambda\left(\tilde{\mu}(s, t) \cdot b^{F} ; \tilde{\mu}(s, t) \cdot(1 ; 2)\right)=t \sqrt{2} \quad \Lambda\left(\tilde{\mu}(s, t) \cdot b^{F} ; \tilde{\mu}(s, t) \cdot(-1 ;-2)\right)=s \sqrt{2} \\
\Lambda\left(\tilde{\mu}(s, t) \cdot b^{F} ; \tilde{\mu}(s, t) \cdot(\infty ; 1)\right)=\sqrt{2} \quad \forall e \in \tau_{*} \backslash\{(1 ; 2) ;(-1 ;-2)\}
\end{gathered}
$$

Notons que $(1 ; 2)=g^{\prime}\left(1 ; \frac{3}{2}\right)$ et $(-1 ;-2)=g^{\prime}(-1 ;-2)$.

L'image par $g^{\prime}$ de l'arête de Farey $e_{1}^{\prime}$ est l'arête $(1 ; 3)$. La formule de Ptolémée donne $\Lambda\left(\tilde{\mu}(s, t) \cdot b^{F} ; \tilde{\mu}(s, t) \cdot(1 ; 3)\right)=(t+1) \sqrt{2}$. Finalement, le support de $\Lambda\left(\tilde{\mu}(s, t) \circ g^{\prime}\right)$ est égal à $\left\{e_{1}^{\prime} ; e_{1} ;\left(1 ; \frac{3}{2}\right)\right\}$ et ses coordonnées sur le support sont:

$$
\Lambda\left(\tilde{\mu}(s, t) \circ g^{\prime}\right)\left(e_{1}^{\prime}\right)=(t+1) \sqrt{2} \quad \Lambda\left(\tilde{\mu}(s, t) \circ g^{\prime}\right)\left(e_{1}\right)=s \sqrt{2} \quad \Lambda\left(\tilde{\mu}(s, t) \circ g^{\prime}\right)\left(\left(1 ; \frac{3}{2}\right)\right)=t \sqrt{2}
$$

On en déduit pour $t=1$ :

$$
\Lambda\left(\tilde{\mu}(s, 1) \circ g^{\prime}\right)\left(e_{1}^{\prime}\right)=2 \sqrt{2} \quad \Lambda\left(\tilde{\mu}(s, 1) \circ g^{\prime}\right)\left(e_{1}\right)=s \sqrt{2} \quad \Lambda\left(\tilde{\mu}(s, 1) \circ g^{\prime}\right)\left(\left(1 ; \frac{3}{2}\right)\right)=\sqrt{2}
$$

D'autre part, le calcul des coordonnées de $\tilde{\mu}(s, t)$ donnent en $t=1$ le même résultat. On en déduit l'égalité $\Lambda\left(\tilde{\mu}(s, 1) \circ g^{\prime}\right)=\Lambda(\tilde{\mu}(s, 2))$. Puisque $\Lambda$ est injective, on a l'égalité dans $\mathcal{G} r$, puis dans $\widehat{\mathcal{G} r}$ puisque ces éléments sont normalisés.

L'égalité $\Lambda(\tilde{\mu}(1, t) \circ g)=\Lambda(\tilde{\mu}(2, t))$ se traite de la même façon et le lemme est démontré.

Considérons l'application suivante:

$$
\tilde{\mu}: \begin{array}{ccc}
I^{2} & \rightarrow \widehat{\mathcal{G} r} / T \\
(s, t) & \mapsto \tilde{\mu}(s, t)
\end{array}
$$

Le lemme précédent implique le:

Corollaire IV.4. L'application $\tilde{\mu}$ est continue et passe au quotient en une application $\mu: \mathbb{T}^{2} \rightarrow \widehat{\mathcal{G} r} / T$ qui transite par $\nu: \mathbb{T}^{2} \rightarrow \mathcal{G} r / F^{\prime}$, c'est à dire $\mu=\rho \circ \nu$, où $\rho$ est l'application $\rho: \mathcal{G} r / F^{\prime} \rightarrow \widehat{\mathcal{G} r} / T$.

Pourquoi transiter par $\nu$ ? Pour montrer que la classe d'homologie de $\mu\left(\mathbb{T}^{2}\right)$ est le générateur de $H_{2}(\widetilde{T})$. En effet, d'après [Ghy-Ser], l'inclusion $F^{\prime} \subset \widetilde{T}$ donne un isomorphisme entre les deuxièmes groupes d'homologie de $F^{\prime}$ et de $\widetilde{T}$. Cet isomorphisme est aussi réalisé par l'application $\rho$. On va montrer que la classe d'homologie, notée $\sigma^{\prime}$ du tore construit dans $\mathcal{G} r / F^{\prime}$ est le générateur de $H_{2}\left(\mathcal{G} r / F^{\prime}\right) \simeq H_{2}\left(F^{\prime}\right)$ (d'après le 
corollaire III.1). Dans $H_{2}\left(F^{\prime}\right)$, l'élément correspondant de ce tore est le cycle $\left(g\right.$ et $g^{\prime}$ commutent) $\sigma^{\prime}=\left(g, g^{\prime}\right)-\left(g^{\prime}, g\right)$.

Pour montrer que $\sigma^{\prime}$ est un générateur de $H_{2}\left(F^{\prime}\right)$, on l'évalue sur une classe de cohomologie dans $H^{2}(T)$, le cocycle de Gobillon-Vey discret. Nous ne faisons ici qu'imiter la démonstration du lemme 4.7. dans l'article [Ghy-Ser]. Ce cocycle s'exprime naturellement dans le modèle linéaire par morceaux du groupe $T$, c'est à dire $P L_{2}\left(\mathbb{S}^{1}\right)$ (voir section I.6).

Pour le définir, on a besoin de la notation suivante. Si $u: \mathbb{S}^{1} \rightarrow \mathbb{R}$ est une fonction admettant des limites latérales en tout point, on notera $\Delta u: \mathbb{S}^{1} \rightarrow \mathbb{R}$ la fonction associée à $u$ définie par $\Delta u(x)=u\left(x^{+}\right)-u\left(x^{-}\right)$. Si $u_{r}$ est la dérivée à droite d'une fonction, on pose pour $(u, v) \in P L_{2}\left(\mathbb{S}^{1}\right) \times P L_{2}\left(\mathbb{S}^{1}\right)$ :

$$
\overline{g v}(u, v)=\sum_{x \in \mathbb{S} 1}\left|\begin{array}{cc}
\log _{2} v_{r}^{\prime} & \log _{2}(u \circ v)_{r} \\
\Delta \log _{2} v_{r}^{\prime} & \Delta \log _{2}(u \circ v)_{r}
\end{array}\right|(x)
$$

Remarquons que cette somme est finie puisque les dérivées des éléments de $P L_{2}\left(\mathbb{S}^{1}\right)$ ont un nombre fini de discontinuité sur le cercle.

Théorème IV.10. (Ghys-Sergiescu [Ghy-Ser] 1987) La fonction $\overline{g v}: P L_{2}\left(\mathbb{S}^{1}\right) \times$ $P L_{2}\left(\mathbb{S}^{1}\right) \rightarrow \mathbb{Z}$ est un cocycle. De plus, si $\alpha$ est le générateur de $H^{2}\left(F^{\prime}, \mathbb{Z}\right)=\mathbb{Z}$, alors $[\overline{g v}]=2 \alpha$.

Il suffit donc d'évaluer $\overline{g v}$ sur $\sigma^{\prime} \in H_{2}\left(F^{\prime}\right)$ et de trouver 2. Pour cela, nous avons indiqué sur la figure IV.2 les éléments $g$ et $g^{\prime}$ et leurs éléments correspondant $u$ et $v$ dans $P L_{2}\left(\mathbb{S}^{1}\right)$. Le calcul porte sur $x=\frac{1}{2} \in \mathbb{R} / \mathbb{Z}$ puisque c'est le seul point commun aux adhérences des supports de $u$ et $v$. On obtient ainsi $\overline{g v}(\sigma)=\overline{g v}(u, v)-\overline{g v}(v, u)=$ $1-(-1)=2$.

On peut résumer ce qui précède par la:

Proposition IV.5. Le cycle $\sigma$ est le générateur de $H_{2}(\widehat{\mathcal{G} r} / T)$.

Corollaire IV.5. Le cocycle $[\mu]$ est nul.

Preuve. - D'après le théorème IV.8, la nullité de $[\mu]$ équivaut à la trivialité du fibré pull-back $\Phi^{*}\left(\left(K \mathbb{S}^{2}\right)_{\bullet}\right)$. Montrons la nullité de sa classe d'Euler $e\left(\Phi^{*}\left(\left(K \mathbb{S}^{2}\right)_{\bullet}\right)\right.$, en l'évaluant sur le cycle générateur $\sigma$ (proposition IV.5). Puisque $\Phi \circ \rho$ est à valeurs dans $J \mathbb{S}^{1}$, et par naturalité de la classe d'Euler, il reste à voir que la restriction de $\left(K \mathbb{S}^{2}\right)$. à $J \mathbb{S}^{1}$ est triviale. Ceci est une conséquence de l'affirmation 3 . du théorème IV.7: $\operatorname{ker} e\left(\left(K \mathbb{S}^{2}\right)_{\bullet}\right) \supset i_{2}^{c o m b}\left(H_{2}\left(J \mathbb{S}^{1}\right)\right)$.

\section{IV.6.c L'image du générateur est un générateur}

A première vue, on doit effectuer le calcul $\psi_{2}(\sigma)$ dans $H_{2}\left(\left(M \mathbb{S}^{2}\right)_{\bullet}\right)=\mathbb{Z}$ et montrer qu'il s'agit d'un générateur. Nous allons conduire le calcul dans l'homologie de $J \mathbb{S}^{1}$ (plus exploitable), et ceci est permis puisque $\sigma$ vit dans $H_{2}\left(F^{\prime}\right)$, et que $F^{\prime}$ a l'homologie 


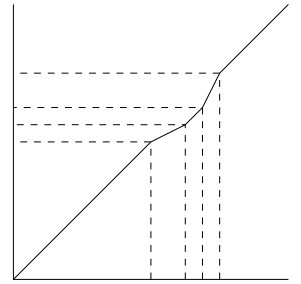

$u$

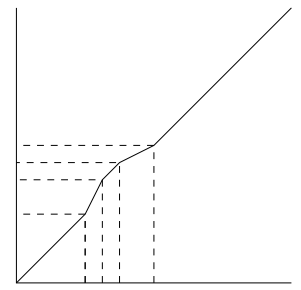

$v$
0
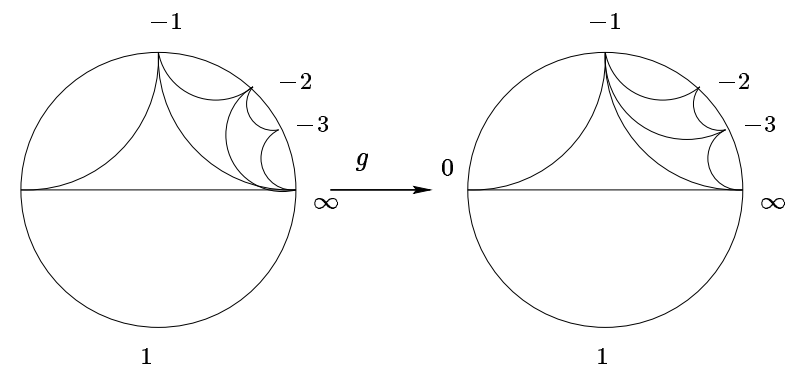

0
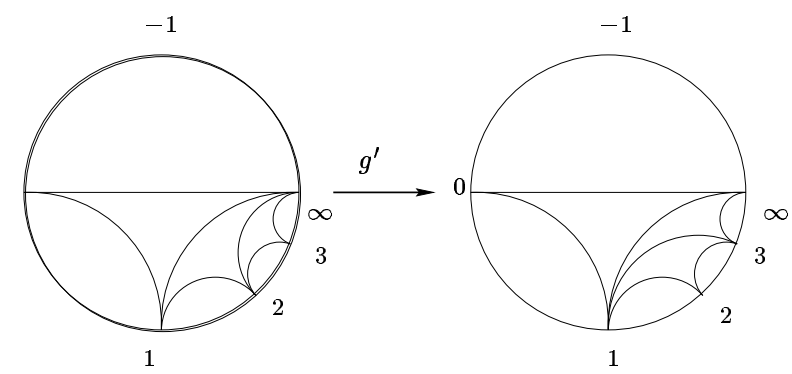

FIG. IV.2 - Le cycle générateur de $H_{2}(\widetilde{T})$.

de $J \mathbb{S}^{1}$. On utilise pour cela l'application $\varphi$ définie en IV.2.b, et ceci est possible grâce à la formule IV.10 reliant les longueurs horocycliques (qui définissent $\varphi$ ) aux fonctions de décalage (qui définissent $\psi$ ). Il faut donc montrer le:

Lemme IV.9. L'image de $\sigma^{\prime}$ par l'application $\varphi_{2}$ (IV.2) est le générateur de $H_{2}\left(J \mathbb{S}^{1}\right)$.

Preuve. - En utilisant le lemme II.4 appliqué au cas $n=3$, pour chaque déformation $g_{s}$ et $g_{s, t}^{\prime}$, on obtient, en ne prenant compte que des longueurs horocycliques non entières:

$$
\varphi(\mu(s, t))=\left[3+\frac{2}{t}, t, 1+\frac{2}{t}, 5+t+s, 3+\frac{2}{s}, s, 5+\frac{2}{s}\right]
$$

l'application donnée par le membre de droite dans IV.15 est homotope à l'application

$$
\begin{array}{rlcc}
\varphi_{h}: & \mathbb{T}^{2} & \rightarrow & J \mathbb{S}^{1} \\
{[s, t]} & \mapsto & {[-t, t,-t, t+s,-s, s,-s]}
\end{array}
$$

Il suffit donc de montrer que $\left(\varphi_{h}\right)_{2}\left(\mathbb{T}^{2}\right)$ est le générateur de $H_{2}\left(J \mathbb{S}^{1}\right)$. Remarquons que l'application $\varphi_{h}$ transite par le tore $\mathbb{T}^{7}$ :

$$
\varphi_{h}: \mathbb{T}^{2} \stackrel{k}{\rightarrow} \mathbb{T}^{7} \stackrel{i^{7}}{\rightarrow} J \mathbb{S}^{1}
$$

où $k(s, t)=(-t, t,-t, t+s,-s, s,-s)$ et $i^{7}\left(\left(\theta_{i}\right)_{i \in<1 ; 7>}\right)=\left[\theta_{i}\right]_{i \in<1 ; 7>} \in J \mathbb{S}^{1}$.

De plus, on a l'égalité:

$$
H_{2}\left(\mathbb{T}^{7}\right)=\oplus_{1 \leq i<j \leq 7} H_{1}\left(\mathbb{S}_{i}^{1}\right) \otimes H_{1}\left(\mathbb{S}_{j}^{1}\right)
$$


où $\mathbb{S}_{i}^{1}$ représente la i-ème coordonnées dans le tore $\mathbb{T}^{7}$, par la formule de Kunneth. Notons $u_{i}$ le générateur de $H_{1}\left(\mathbb{S}_{i}^{1}\right)$. Chaque terme $u_{i} \otimes u_{j}$ est envoyé par $i_{2}^{7}$ sur le générateur, notons le $\gamma$ de $H_{2}\left(J \mathbb{S}^{1}\right)$. Le calcul se fait dans l'homologie de $\mathbb{T}^{7}$, et on obtient alors:

$$
\begin{aligned}
& k_{2}([s, t])=[-t, t]+[-t,-t]+[-t, t+s]+[-t,-s]+[-t, s]+[-t,-s] \\
& +\quad[t,-t]+[t, t+s]+[t,-s]+[t, s]+[t,-s] \\
& +\quad[-t, t+s]+[-t,-s]+[-t, s]+[-t,-s] \\
& +\quad[t+s,-s]+[t+s, s]+[t+s,-s] \\
& + \\
& + \\
& \begin{aligned}
{[-s, s]+} & {[-s,-s] } \\
& {[s,-s] }
\end{aligned}
\end{aligned}
$$

L'application $k^{1,2}:(s, t) \in \mathbb{T}^{2} \mapsto(-t, t) \in \mathbb{S}_{1}^{1} \times \mathbb{S}_{2}^{1}$ envoie le cycle fondamental du tore sur 0 , puisque son image est un cercle. Il en est de même pour les applications $k^{5,6}$, $k^{5,7}$ et $k^{6,7}$ avec les notations évidentes.

L'application $k^{1,3}:(s, t) \in \mathbb{T}^{2} \mapsto(-t, t+s) \in \mathbb{S}_{1}^{1} \times \mathbb{S}_{3}^{1}$ se relève en un automorphisme de $\mathbb{R}^{2}$ de déterminant 1 . Elle envoie donc le cycle fondamental du tore sur $u_{1} \otimes u_{4}$. En faisant de même pour les autres termes, on obtient finalement

$$
\begin{aligned}
& k_{2}([s, t])=0+0+u_{1} \otimes u_{4}-u_{1} \otimes u_{5}+u_{1} \otimes u_{6}-u_{1} \otimes u_{7} \\
& +\quad 0-u_{2} \otimes u_{4}+u_{2} \otimes u_{5}-u_{2} \otimes u_{6}+u_{2} \otimes u_{7} \\
& +\quad+u_{3} \otimes u_{4}-u_{3} \otimes u_{5}+u_{3} \otimes u_{6}-u_{3} \otimes u_{7} \\
& +\quad+u_{4} \otimes u_{5}-u_{4} \otimes u_{6}+u_{4} \otimes u_{7} \\
& +\quad 0+0 \\
& +\quad 0
\end{aligned}
$$

Mais puisque chaque $u_{i} \otimes u_{j}$ s'envoie par $i^{7}$ sur $\gamma$, on a l'égalité, après simplification, $\varphi_{2}\left(\sigma^{\prime}\right)=\gamma$, et le lemme est démontré.

Considérons à présent le diagramme commutatif suivant:

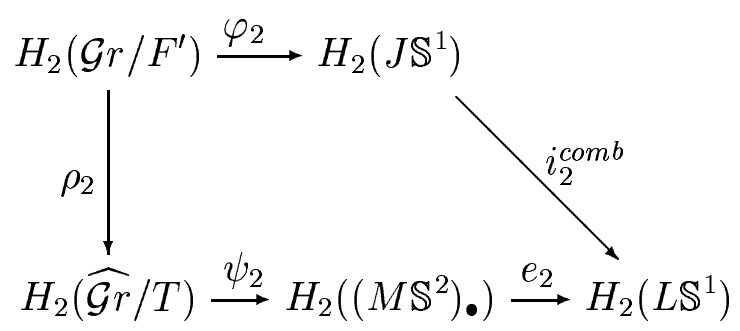

où $e$ est l'application composée $\left(M \mathbb{S}^{2}\right) \bullet \rightarrow\left(K \mathbb{S}^{2}\right) \bullet \rightarrow\left(L \mathbb{S}^{1}\right)_{\bullet}$.

Corollaire IV.6. $\psi_{2}$ fournit un isomorphisme entre $H_{2}(\widehat{\mathcal{G r}} / T)$ et $H_{2}\left(\left(M \mathbb{S}^{2}\right)_{\bullet}\right)$.

Preuve. - Notons $\alpha=\psi_{2}(\sigma)$ et $\alpha_{0}$ le générateur de $H_{2}\left(\left(M \mathbb{S}^{2}\right) \bullet\right)=\mathbb{Z}$ (corollaire IV.2), puis $l \in \mathbb{Z} \mid \alpha=l \alpha_{0}$. Le diagramme commutatif, où chaque flèche allant vers le 
bas représente une équivalence d'homotopie:

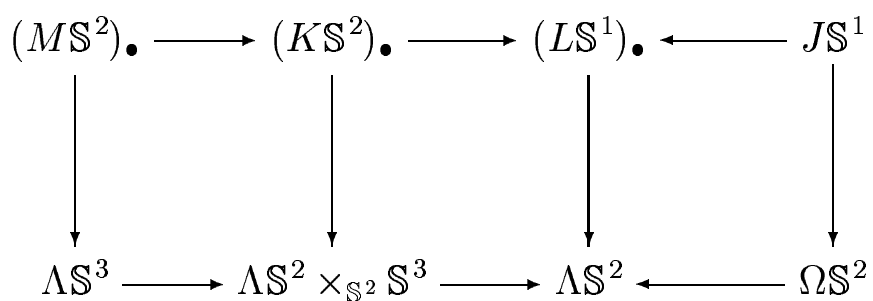

indique d'une part que $\delta=e_{2}(\alpha)$ est inclus dans

$$
\mathbb{Z} \oplus\{0\} \subset \mathbb{Z} \oplus \mathbb{Z}=H_{2}\left(L \mathbb{S}^{1}\right)
$$

et d'autre part que $\mathbb{Z} \oplus\{0\}=\operatorname{Im}\left(i_{2}^{\text {comb }}\right)$. Soit $e_{2}\left(\alpha^{\prime}\right)=(k, 0)$. Puisque $\varphi_{2}\left(\sigma^{\prime}\right)$ est le générateur de $H_{2}\left(J \mathbb{S}^{1}\right)$, le diagramme précédent implique que son image par $i_{2}^{\text {comb }}$ est $(0,1)$. Le diagramme précédent en homologie de degré 2 donne alors l'égalité $e_{2}(\alpha)=$ $(0,1)$, ce qui implique que $l k=1$. Ainsi, $\alpha$ est le générateur de $H_{2}\left(\left(M \mathbb{S}^{2}\right) \bullet\right)$.

Passons maintenant à l'homologie en dimension 3.

\section{IV.6.d Le générateur en dimension 3}

Concernant le générateur de degré 3, l'isomorphisme

$$
\psi_{2}: H_{2}(\widehat{\mathcal{G r}} / T) \rightarrow H_{2}\left(\left(M \mathbb{S}^{2}\right)_{\bullet}\right)
$$

passe au produit puis au quotient par $\mathbb{S}^{1}$ (car le tore $\mathbb{T}^{2}$ est normalisé) en l'application suivante:

$$
(\psi \times i d)_{2}: H_{2}\left(\widehat{\mathcal{G} r} / T \times_{\mathbb{S}_{1}} E \mathbb{S}^{1}\right) \rightarrow H_{2}\left(\left(M \mathbb{S}^{2}\right) \cdot \times_{\mathbb{S}^{1}} E \mathbb{S}^{1}\right)
$$

On sature cette application par la fibre $\mathbb{S}^{1}$ pour donner le diagramme commutatif suivant:

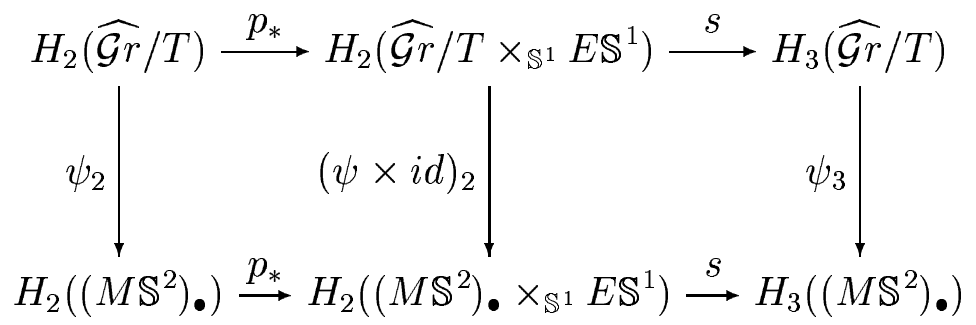

On a effectué en homologie l'opération duale de l'intégration par la fibre en cohomologie. Le composé en bas $s \circ p_{*}$ est un isomorphisme d'après [Ghy-Ser]) et le corollaire IV.2. De plus le corollaire III.2 entraine $H_{3}(\widehat{\mathcal{G} r} / T)=\mathbb{Z}$. Via le diagramme précédent, on en déduit que $\psi_{3}$ est un isomorphisme. 


\section{IV.6.e En toute dimension}

Pour passer à l'isomorphisme en toute dimension, on souhaiterait utiliser la structure de Hopf sur l'homologie relative à la structure de H-espace associé à $\Lambda \mathbb{S}^{3}$. Malheureusement, nous ne possédons pas de $\mathrm{H}$-structure naturelle sur $\widehat{\mathcal{G} r} / T$. Pour contourner ce problème, nous utilisons la cohomologie entière qui coïncide avec l'homologie entière puisque les groupes d'homologie que nous envisageons sont tous libres. La structure de produit exterieur de la cohomologie est toujours définie et est naturellement préservée par les applications.

On rappelle rapidement la structure de l'anneau de cohomologie de $\widetilde{T}$ [Ghy-Ser]. Soit $\Lambda(u)$ la $\mathbb{Z}$-algèbre extérieure engendrée par un générateur de degré impair $u$ et $\Gamma(x)$ l'anneau gradué des puissances divisées, engendré par un générateur $x$ de degré pair, c'est à dire l'anneau libre engendré par le système de générateurs $\left(x_{n}\right)_{n \geq 1}$ avec $x_{1}=x$, les autres éléments étant obtenus par récurrence grâce aux égalités $x_{n} \cdot x_{p}=\frac{(n+p) !}{n ! p !} x_{n+p}$. Rappelons que $H^{*}(\widetilde{T} ; \mathbb{Z})=\Gamma[\alpha] \otimes \Lambda(u)$ ou $\alpha$ est de degré 2 et $u$ de degré 3 . De même, on notera $H^{*}\left(\left(M \mathbb{S}^{2}\right)_{\bullet} ; \mathbb{Z}\right)=\Gamma[\beta] \otimes \Lambda(v)$.

Théorème IV.11. L'application $\psi$ fournit un isomorphisme en homologie entière.

Preuve. - Puisque l'homologie de $\widetilde{T}$ (resp. $\Lambda \mathbb{S}^{3}$ ) ne contient que des groupes libres (isomorphes à $\mathbb{Z}$ ou nuls), elle coïncide avec sa cohomologie. D'après ce qui précède, $\psi$ envoie $\alpha$ sur $\beta$ et $u$ sur $v$. Pour la dimension $2 n$ avec $n \geq 1$, notons que la structure d'algèbre des puissances divisées, de part et d'autre implique que $\frac{\alpha^{n}}{n !}$ (resp. $\frac{\beta^{n}}{n !}$ ) est le générateur de $H^{2 n}(\widehat{\mathcal{G r}} / \widetilde{T})$ (resp. $H^{2 n}\left(\left(M \mathbb{S}^{2}\right)\right.$ •)). Par ailleurs, l'application $\psi$ présèrve la structure multiplicative des anneaux de cohomologie. Ainsi, on a $\beta^{n}=\psi^{*}\left(\alpha^{n}\right)=$ $n ! \psi^{*}\left(\frac{\alpha^{n}}{n !}\right)$. Par conséquent, $\psi^{*}\left(\frac{\alpha^{n}}{n !}\right)=\frac{\beta^{n}}{n !}$, ce qui montre l'isomorphisme en dimension paire. L'argument est identique en dimension impaire, et ceci termine la démonstration du théorème.

\section{Corollaire IV.7.}

$$
\varphi^{\prime} \times i d: \widehat{\mathcal{G} r} / T \times_{\mathbb{S}^{1}} E \mathbb{S}^{1} \rightarrow \mathcal{L S}^{3}
$$

est une équivalence en homologie entière.

Preuve. - Il suffit d'appliquer le lemme des cinq sur le diagramme commutatif résultant des suites de Gysin associées aux fibrés en cercle $\mathbb{S}^{1} \rightarrow \widehat{\mathcal{G r}} / T \times E \mathbb{S}^{1} \rightarrow \widehat{\mathcal{G r}} /$ $T \times \mathbb{S}^{1} E \mathbb{S}^{1}$ et $\mathbb{S}^{1} \rightarrow \Lambda \mathbb{S}^{3} \times E \mathbb{S}^{1} \rightarrow \mathcal{L} \mathbb{S}^{3}$

On aura remarqué que $\psi$ et $\psi \times i d$ fournissent des versions géométriques des applications $b$ et $c$ du théorème IV.1. 


\section{IV.7 Le cas du groupe $F^{\prime}$ : une nouvelle preuve}

\section{IV.7.a Une structure multiplicative sur $B F^{\prime}$}

Nous construisons ici un produit sur l'espace $\mathcal{G} r$ qui passe au quotient pour donner un produit sur $\mathcal{G} r / F^{\prime}$. On utilise une autre normalisation de $\mathcal{G} r$ analogue à la normalisation des bracelets introduite au paragraphe II.1.d. Celle-ci est définie comme suit:

$$
\mathcal{G} r=\{f \in C P P \mid b k(f) \subset \widehat{\mathbb{Q}} \text { et } \overline{\operatorname{Supp}(f)} \subset] \infty ; 0]\}
$$

et

$$
F^{\prime}=\{\gamma \in T \mid \overline{\operatorname{Supp}(\gamma)} \subset] \infty ; 0[\}
$$

D'une part, ce nouvel $F^{\prime}$ est isomorphe à l'ancien défini au paragraphe III.6. D'autre part, il agit par composition à droite sur cette version normalisé de $\mathcal{G} r$.

Pour définir un produit sur $\mathcal{G} r$, on utilise les éléments de $P S L_{2} \mathbb{Z}$ suivants:

$$
\begin{gathered}
A: x \in \mathbb{R} \cup\{\infty\} \mapsto \frac{x}{2 x+1} \in \mathbb{R} \cup\{\infty\} \\
B: x \in \mathbb{R} \cup\{\infty\} \mapsto x+2 \in \mathbb{R} \cup\{\infty\}
\end{gathered}
$$

qui sont des éléments paraboliques vérifiant

$$
\begin{array}{clrl}
A(0) & =0 & & A\left(-\frac{1}{2}\right)=\infty \\
B(\infty) & =\infty & B(-2) & =0
\end{array}
$$

Si $f \in \mathcal{G} r$, on note $f^{A}$ (resp. $f^{B}$ ) le conjugué de $f$ par $A^{-1}$ (resp. $B^{-1}$ ).

Proposition IV.6. Si $f \in \mathcal{G}$ r, alors $f^{A}$ et $f^{B}$ sont des éléments de $\mathcal{G} r$ vérifiant $\overline{\operatorname{Supp}\left(f^{A}\right)} \subset\left[-\frac{1}{2} ; 0\right]$ et $\overline{\operatorname{Supp}\left(f^{B}\right)} \subset[\infty ;-2]$.

Preuve. - Puisque $A \in P S L_{2} \mathbb{Z}, f^{A}$ est à points de coupure rationnels. L'affirmation évidente $\overline{\operatorname{Supp}\left(f^{A}\right)} \subset\left[-\frac{1}{2} ; 0\right]$ permet de conclure que $f^{A}$ est normalisé. Le même argument est valable pour $f^{B}$.

Nous sommes en mesure de définir une opération produit sur l'espace $\mathcal{G} r$.

Définition IV.7.1. (Produit) Soit $\times$ l'application suivante:

$$
\times:(f, g) \in \mathcal{G} r^{2} \mapsto f \times g=f^{B} \circ g^{A} \in \mathcal{G} r
$$

Puisque $\operatorname{Supp}\left(f^{B}\right) \cap S u p p\left(g^{A}\right)=\emptyset$, le produit $f \times g$ est encore à points de coupure rationnels. Il est aussi normalisé puisque $\operatorname{Supp}(f \times g) \subset S u p p\left(f^{B}\right) \cup \operatorname{Supp}\left(g^{A}\right)$. On a ainsi muni $\mathcal{G} r$ d'une structure de produit. Remarquons enfin que $f \times g_{\left[\left[-2 ;-\frac{1}{2}\right]\right.}=i d$, de sorte que le bracelet de Ford $b_{2}$ est fixé par $f \times g$. 
On montre à présent que ce produit passe au quotient par $F^{\prime}$.

Proposition IV.7. (Passage au quotient) $S i\left(f, g, \gamma, \gamma^{\prime}\right) \in \mathcal{G} r^{2} \times F^{2}$, alors il existe $\gamma^{\prime \prime} \in F^{\prime}$ tel que

$$
(f \circ \gamma) \times\left(g \circ \gamma^{\prime}\right)=(f \times g) \circ \gamma^{\prime \prime}
$$

Preuve. - Remarquons que $\gamma \in F^{\prime}$ implique que $\gamma^{A}$ et $\gamma^{B}$ sont aussi des éléments du groupe $F^{\prime}$. On a $(f \circ \gamma) \times\left(g \circ \gamma^{\prime}\right)=f^{B} \circ \gamma^{B} \circ g^{A} \circ \gamma^{A}$. Puisque $\operatorname{Supp}\left(\gamma^{B}\right) \cap S u p p\left(g^{A}\right)=\emptyset$, les éléments $\gamma^{B}$ et $g^{A}$ commutent. On obtient donc l'égalité recherchée en posant $\gamma^{\prime \prime}=\gamma^{B} \circ \gamma^{A}$.

Ce produit passe donc au quotient. On le note de la même façon sur $\mathcal{G} r / F^{\prime}$. Notons qu'il n'est pas associatif à homotopie près, puisque $F^{\prime}$ n'est pas abélien. Or, le groupe fondamental d'un $H$-espace (espace muni d'un produit associatif à homotopie prés) est abélien.

\section{IV.7.b Multiplicativité de $B F^{\prime} \rightarrow \Omega \mathbb{S}^{2}$}

On commence par définir les sous-ensembles de $S_{n}$ intervenant dans la suite. On note $S_{n}^{-}=S_{n} \cap \mathbb{R}_{-}=S_{n}^{B} \cup S_{n}^{0} \cup S_{n}^{A}$, où $\left.S_{n}^{B}=S_{n} \cap\left[0 ;-\frac{1}{2}\right], S_{n}^{0}=S_{n} \cap\right]-\frac{1}{2} ;-2[$ et $S_{n}^{A}=S_{n} \cap[-2 ; \infty]$. L'homographie $A^{-1}\left(\operatorname{resp} . B^{-1}\right)$ fournit une bijection de l'ensemble $S_{n}^{-}$vers $S_{n+2}^{A}\left(\right.$ resp. $\left.S_{n+2}^{B}\right)$.

Considérons à présent l'application $\varphi: \mathcal{G} r \rightarrow J \mathbb{S}^{1}$ définie au IV.2. Rappelons que le modèle de James possède la structure de H-espace induite par la concaténation des mots.

Lemme IV.10. L'application $\varphi$ est multiplicative.

Preuve. - Soient $f$ et $g$ deux éléments de $\mathcal{G} r_{n}$. Puisque $A^{-1}$ (resp. $B^{-1}$ ) envoyent $S_{n}^{-} \operatorname{sur} S_{n+2}^{A}\left(\operatorname{resp} . S_{n+2}^{B}\right)$, on a $b k(f \times g)=b k\left(f^{B}\right) \cup b k\left(g^{A}\right) \subset S_{n+2}^{-}$. Ainsi, le produit de $f$ et $g$ est un élément de $\mathcal{G} r_{n+2}$. De plus, puisque les applications $A$ et $B$ fixent globalement le bracelet de Ford, on a les égalités:

$$
\begin{array}{ll}
l_{x}\left((f \times g) \cdot b_{n+2}\right)=l_{B \cdot x}\left(f \cdot b_{n}\right) & \forall x \in S_{n+2}^{B} \\
l_{x}\left((f \times g) \cdot b_{n+2}\right)=l_{A \cdot x}\left(g \cdot b_{n}\right) & \forall x \in S_{n+2}^{A}
\end{array}
$$

Enfin, si $x \in S_{n+2}^{0}$, alors on a $l_{x}\left((f \times g) \cdot b_{n+2}\right)=l_{x}\left(b_{n+2}\right) \in \mathbb{Z}$, car $f \times g$ est à support disjoint de l'intervalle $]-2 ;-\frac{1}{2}\left[\right.$. On obtient donc l'égalité dans $J \mathbb{S}^{1}$

$$
\varphi_{n+2}(f \times g)=\varphi_{n}(f) \cdot \varphi_{n}(g)
$$

On en déduit le lemme. 


\section{IV.7.c Homologie de $F^{\prime}$}

Dans [Bro-Geo], les auteurs construisent un complexe cubique sur lequel le groupe $F$ agit. L'étude de l'action de $F$ sur ce complexe permet de calculer l'homologie de $F$ :

Théorème IV.12. (Brown-Geoghegan [Bro-Geo], Stein [Ste]) Pour tout $n \geq 0$, le groupe $H_{n}(F ; \mathbb{Z})$ est isomorphe à $\mathbb{Z} \oplus \mathbb{Z}$.

Soit $F^{+}$le sous-groupe de $F$ des éléments dont le germe à gauche en 1 est l'identité et considérons les morphismes de groupes :

$$
\begin{gathered}
\varphi_{l}: f \in F \mapsto \log _{2} f_{l}^{\prime}(1) \in \mathbb{Z} \\
\varphi_{r}: f \in F^{+} \mapsto \log _{2} f_{r}^{\prime}(0) \in \mathbb{Z}
\end{gathered}
$$

Partant du théorème IV.12, nous utilisons les extensions de groupes suivantes:

$$
\begin{gathered}
0 \rightarrow F^{+} \rightarrow F \stackrel{\varphi_{l}}{\rightarrow} \mathbb{Z} \rightarrow 0 \\
0 \rightarrow F^{\prime} \rightarrow F^{+} \stackrel{\varphi_{r}}{\rightarrow} \mathbb{Z} \rightarrow 0
\end{gathered}
$$

En appliquant les suites de Wang en homologie associées à ces extensions, on obtient le résultat suivant:

Proposition IV.8. $\forall k \geq 0$, on a les égalités:

$$
H_{2 k}\left(F^{\prime} ; \mathbb{Z}\right)=\mathbb{Z} \text { et } H_{2 k+1}\left(F^{\prime} ; \mathbb{Z}\right)=0
$$

Preuve. - Commençons par la première extension. Soit $s$ une section de $\varphi_{l}$. La conjugaison par $s(1)$ dans $F$ présèrve le sous-groupe $F^{+}$et donne naissance à un automorphisme de $F^{+}$noté $A^{s}$. L'application en homologie

$$
H_{k}\left(F^{+}\right) \stackrel{A_{k}^{s}-i d_{k}}{\rightarrow} H_{k}\left(F^{+}\right)
$$

ne dépend pas de la section $s$ et donne la suite exacte de Wang en homologie:

$$
\cdots \rightarrow H_{k+1}(F) \rightarrow H_{k}\left(F^{+}\right) \stackrel{A_{k}^{s}-i d_{k}}{\rightarrow} H_{k}\left(F^{+}\right) \rightarrow H_{k}(F) \rightarrow \cdots
$$

Les morphismes $A_{k}^{s}-i d_{k}$ sont nuls. En effet, prenons $\gamma$ un cycle en homologie du type $\left[f_{1}|\cdots| f_{k}\right]$. Notons $x_{0}=S u p\{x \in] 0 ; 1\left[\cap\left(\cup_{i \in<1 ; k>} \operatorname{Supp}\left(f_{i}\right)\right)\right\}$. On a clairement $x_{0}<1$ puisque les éléments $f_{i}$ ont leurs germes égaux à l'identité à gauche de 1 . Il n'est pas difficile de construire un élément $g_{0} \in F$ à support contenu dans l'intervalle ]$\left.x_{0} ; 1\right]$ et dont la dérivée à gauche de 1 vaut 2 . L'élément $g_{0}$ définit alors une section $s$ de $\varphi_{l}$ en posant $s(1)=g_{0}$. Comme $\operatorname{Supp}\left(g_{0}\right) \cap S u p p\left(f_{i}\right)=\emptyset$ pour tout $i$, on en déduit que le morphisme $A_{k}^{s}-i d_{k}$ évalué sur le cycle $\gamma$ est nul. Ce morphisme ne dépendant pas de $s$, on en conclut qu'il est nul. 
Ecrivons la suite de Wang associée à l'extension $\varphi_{l}$ :

$$
\cdots \mathbb{Z} \oplus \mathbb{Z} \stackrel{a_{k}}{\rightarrow} H_{k}\left(F^{+}\right) \stackrel{0}{\rightarrow} H_{k}\left(F^{+}\right) \stackrel{b_{k}}{\rightarrow} \mathbb{Z} \stackrel{c_{k}}{\rightarrow} H_{k-1}\left(F^{+}\right) \stackrel{0}{\rightarrow} H_{k-1}\left(F^{+}\right) \cdots
$$

Puisque $\operatorname{Ker}\left(b_{k}\right)=\operatorname{Im}(0)=0, H_{k}\left(F^{+}\right)$est un sous-groupe de $\mathbb{Z} \oplus \mathbb{Z}$. Il est donc sans-torsion. De plus, $\operatorname{Ker}\left(c_{k}\right)=\operatorname{Im}\left(b_{k}\right)=H_{k}\left(F^{+}\right)$. Par conséquent $c_{k}$ factorise en un isomorphisme de $\mathbb{Z} \oplus \mathbb{Z} / H_{k}\left(F^{+}\right)$vers $\mathbb{Z}$. On en déduit que $H_{k}\left(F^{+}\right)$est isomorphe à $\mathbb{Z}$.

Le même argument appliqué à la seconde extension de groupes permet de conclure la démonstration.

On en vient à la deuxième démonstration de l'équivalence d'homologie entre $B F^{\prime}$ et $\Omega \mathbb{S}^{3}$.

\section{IV.7.d Deuxième démonstration}

On connait à présent l'homologie de $F^{\prime}$, mais nous n'avons à priori aucune information sur une éventuelle structure multiplicative existante sur cette homologie. Par contre, nous connsaissons la structure d'algebre de l'homologie de $\Omega \mathbb{S}^{3}$ dans celle de $\Omega \mathbb{S}^{2}$. Si $\sigma^{\prime}$ (resp. $\gamma$ ) désigne le générateur de degré 2 de l'homologie de $\mathcal{G} r / F^{\prime}$ (resp. $\left.J \mathbb{S}^{1}\right)$, on a $\varphi_{2}\left(\sigma^{\prime}\right)=\gamma$ d'après le lemme IV.9.

Pour montrer que $\varphi_{2 n}$ est un isomorphisme, on utilise un diagramme commutatif qui traduit l'associativité de "l'image" de $\times \operatorname{par} \varphi_{n}$.

Notons $\times^{n}\left(f_{1}, \cdots, f_{n}\right)=f_{1} \times\left(f_{2} \times\left(\cdots \times f_{n}\right) \cdots\right)$ et $c o^{n}\left(x_{1}, \cdots x_{n}\right)=x_{1} \cdot x_{2} \cdots x_{n}$, la concaténation des mots. La proposition II.2 entraine l'égalité dans $J \mathbb{S}^{1}$ :

$$
\varphi\left(\times_{n}\left(f_{1}, \cdots, f_{n}\right)\right)=\varphi\left(f_{1}\right) \cdot \varphi\left(f_{2}\right) \cdots \varphi\left(f_{n}\right)
$$

Ceci se traduit par le diagramme commutatif:

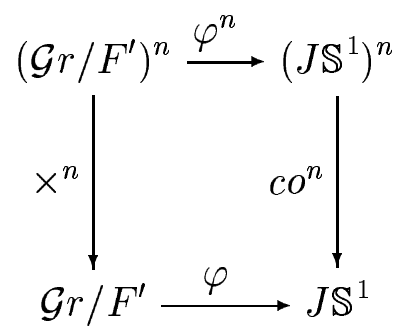

qui donne en homologie le diagramme suivant:

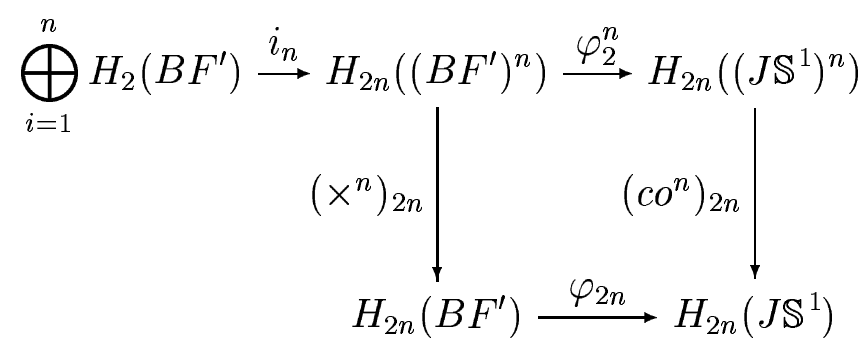


Considérons $\alpha=\oplus_{i=1}^{n} \sigma_{i}^{\prime} \in \bigoplus_{i=1}^{n} H_{2}\left(B F^{\prime}\right)$, où $\sigma_{i}^{\prime}$ est une copie de $\sigma^{\prime}$, et $\sigma^{n}$ son image par $\left(\times^{n}\right)_{2 n} \circ i_{n}$. En utilisant la commutativité du diagramme précédent et la structure de Hopf sur l'homologie de $J \mathbb{S}^{1}$, on obtient successivement

$$
\varphi_{2 n}\left(\sigma^{n}\right)=c o_{2 n}^{n} \circ\left(\varphi_{2}\right)^{n} \circ i_{n}\left(\oplus_{i=1}^{n} \sigma_{i}^{\prime}\right)=\left[\varphi_{2}\left(\sigma^{\prime}\right)\right]^{n}
$$

On en déduit que $\varphi_{2 n}$ est un morphisme surjectif de $\mathbb{Z}$ dans $\mathbb{Z}$ et l'argument utilisé dans la démonstration du corollaire IV.6 permet de conclure l'injectivité de $\varphi_{2 n}$.

Puisque en dimension impaire, les homologies sont triviales de part et d'autre, on en déduit que $\varphi$ réalise un isomorphisme en homologie entière entre $\mathcal{G} r / F^{\prime}$ et $\Omega \mathbb{S}^{3}$.

- Remarque: On en déduit directement la structure multiplicative sur l'homologie de $F^{\prime}$. En effet, la démonstration précédente montre également que $\sigma^{n}$ est le générateur de $H_{2 n}\left(F^{\prime}\right)$.

La connaissance d'un cycle générateur $\sigma^{\prime}=\left[\mathbb{T}^{2}\right]$ de l'homologie de $\mathcal{G} r / F^{\prime}$ permet de construire explicitement un cycle torique $\left[\mathbb{T}^{2 n}\right]$ (c'est un tore car $f^{B}$ et $g^{A}$ commutent), générateur de l'homologie en degré $2 n$. Plus précisément, ce générateur est une sous-variété donné comme quotient d'un $2 n$-cube $I^{2 n} \subset \mathcal{G} r$, par $2 n$-mouvements élémentaires le long d'arêtes de Farey à supports deux à deux disjoints. 


\section{Bibliographie}

[Abi] W. Abikoff, The real analytic theory of Teichmüller space, L. N. M. 820, Springer Verlag, 1980.

[Bea] A. F. BeARDon, The geometry of discrete groups, Graduate Texts in Mathematics, 91. Springer-Verlag, New York, 1983.

[Bri] M. G. BRIN, The chameleon groups of Richard J. Thompson: automorphisms and dynamics, Inst. Hautes Études Sci. Publ. Math. No. 84 (1996), 5-33 (1997).

[Bro-Geo] K. S. Brown, R. Geoghegan, An infinite-dimensional torsion-free $\mathrm{FP}_{\infty}$ group, Invent. Math. 77 (1984), no. 2, 367-381.

[Can-Flo-Par] J. CAnnon, W. Floyd, W. PARRY, Introductory notes on Richard Thompson's groups, L'Ens. Math. 42 (1996), 215-256.

[Coh] R. L. Cohen, A model for the free loop space of a suspension, Lect. Notes Math. 1286 (1987) 193-207.

[Die-Sch] J. Dieudonné, L. Schwartz, La dualité dans les espaces $\mathcal{F}$ et $\mathcal{L} \mathcal{F}$, Ann. Inst. Fourier 1 (1949), 61-100.

[Dyd] J. DYDAK, A simple proof that pointed FANR-spaces are regular fundamental retracts of ANR's, Bull. Acad. Polon. Sci. Sér. Sci. Math. Astronom. Phys. 25 (1977), no. 1, 55-62.

[Ear-Eel] C. J. EARLE, J. EELs, On the differential geometry of Teichmüller spaces, J. Analyse Math. 19 (1967), 35-52.

[Gar-Har] F. P. Gardiner, W. J. HARvey, Universal Teichmüller space, preprint (2000).

[Gar-Lak] F. P. GARDIneR, N. LAKIC, Quasiconformal Teichmüller theory, Mathematical Surveys and Monographs, 76. American Mathematical Society, Providence, RI, 2000.

[Ghy-Ser] E. Ghys, V. Sergiescu, Sur un groupe remarquable de difféomorphismes du cercle, Comment. Math. Helv. 62 (1987), 185-239.

[God] C. Godbillon, Feuilletages. (French) [Foliations] Études géométriques. [Geometric studies] With a preface by G. Reeb. Progress in Mathematics, 98. Birkhäuser Verlag, Basel, 1991.

[Gre1] P. Greenberg, Pseudogroups from group actions, Amer. J. Math. 109 (1987), no. 5, 893-906.

[Gre2] P. Greenberg, Pseudogroups of $C^{1}$ piecewise projective homemorphisms, Pac. J. Math. 129 n.1 (1987), 67-74. 
[Gre3] P. Greenberg, Les espaces de bracelets, les complexes de Stasheff et le groupe de Thompson, Bol. Soc. Mat. Mexicana, Vol 37 (1992), 189-201.

[Gre4] P. Greenberg, Primer on CPP geometry, preprint in Strukturen in der Mathematik 92-006 Universität Bielefeld (1992).

[Gre-Ser] P. Greenberg, V. Sergiescu, An acyclic extension of the Braid group, Comment. Math. Helv. 66 (1991), 109-138.

[Hin] N. Hingston, An equivariant model for the free loop space of $\mathbb{S}^{n}$, Amer. J. Math. 114 (1991), 139-155.

[Imb] M. ImBeRT, Sur l'isomorphisme du groupe de Richard Thompson avec le groupe de Ptolémée, Lon. Math. Soc. L.N.S. 243 (1997), 313-324.

[Jam] I. JAmes, Reduced product spaces, Ann. of Math. 62 (1955), 170-197.

[Loc-Sch] P. Lochak, L. Schneps, The universal Ptolemy-Teichmüller groupoid, Geometric Galois actions, 2, 325-347, London Math. Soc. Lecture Note Ser., 243, Cambridge Univ. Press, Cambridge, 1997.

[Mal-Pen] F. Malikov, R. C. Penner, The Lie algebra of homeomorphisms of the circle, Adv. Math. 140 (1998), no. 2, 282-322.

[Mat] J. N. Mather, Integrability in codimension 1, Comment. Math. Helv. 48 (1973), 195-233.

[McK-Tho] R. Mckenzie, R. J. Thompson, An elementary construction of unsolvable word problems in group theory. Word problems: decision problems and the Burnside problem in group theory (Conf., Univ. California, Irvine, Calif. 1969; dedicated to Hanna Neumann), pp. 457-478. Studies in Logic and the Foundations of Math., Vol. 71, North-Holland, Amsterdam, 1973.

[Pen1] R. C. Penner, The decorated Teichmüller spaces of punctured surfaces, Comm. Math. Phys. 113 (1987), 299-339.

[Pen2] R. C. Penner, Universal Constructions in Teichmüller spaces, Adv. in Math. 98 (1993), 143-215.

[Pen3] R. C. Penner, The universal Ptolemy group and its completions, Geometric Galois actions, 2, 293-312, London Math. Soc. Lecture Note Ser., 243, Cambridge Univ. Press, Cambridge, 1997.

[Rad] H. RADEMACHER, Lectures on elementary number theory, Blaisdell, New York (1964).

[Roy] H. Royden, Automorphisms and isometries of Teichmüller space, Ann. of Math. Studies 66 (1971), 369-384.

[Sta] J. D. StaSheff, Homotopy associativity of H-spaces. I, II. Trans. Amer. Math. Soc. 108 (1963), 275-292; ibid. 1081963 293-312.

[Ste] M. Stein, Groups of piecewise linear homeomorphisms, Trans. Amer. Math. Soc. 332 (1992), no. 2, 477-514.

[Tho] R. J. Thompson Handwritten Notes.

[Tsu] T. Tsubor, A Characterization of Godbillon-Vey invariant. Sūgaku Expositions Vol. 8 (1995), no. 2, $165-182$. 